\title{
HYDROLOGIC MONITORING IN THE AREA OF THE TENNESSEE-TOMBIGBEE WATERWAY, MISSISSIPPI-ALABAMA, FISCAL YEAR 1989
}

By Fred Morris III

U.S. GEOLOGICAL SURVEY

Open-File Report 91-520

Prepared in cooperation with the

U.S. ARMY CORPS OF ENGINEERS MOBILE DISTRICT

Jackson, Mississippi 


\section{U.S. DEPARTMENT OF THE INTERIOR}

MANUEL LUJAN, JR., Secretary

U.S. GEOLOGICAL SURVEY

Dallas L. Peck, Director

For additional information write to:

U.S. Geological Survey

Water Resources Division

100 W. Capitol Street, Suite 710

Jackson, MS 39269
U.S. Geological Survey

Water Resources Division

520 19th Avenue

Tuscaloosa, AL 35401

Copies of this report can be purchased from:

U.S. Geological Survey

Books and Open-File Reports

Box 25425, Federal Center

Denver, CO 80225 


\section{CONTENTS}

Page

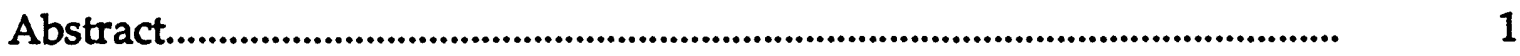

Program changes for 1989.................................................................................

Hydrologic monitoring.........................................................................................

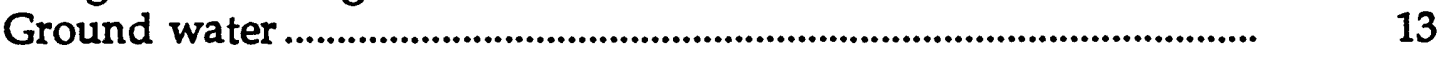

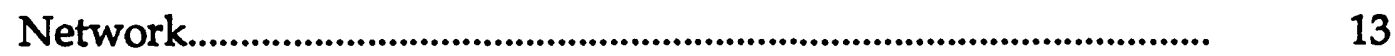

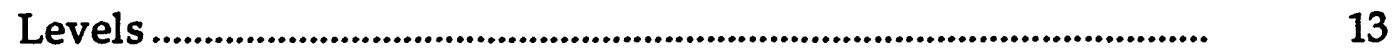

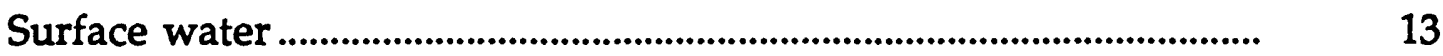

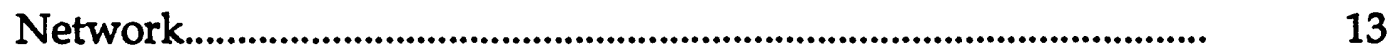

Stage and discharge ........................................................................ 13

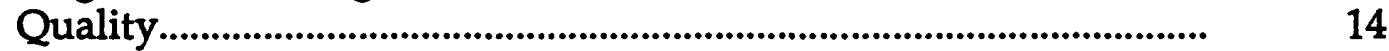

Quality assurance.................................................................................................. 16

Ground-water levels............................................................................... 16

Surface-water stage and discharge............................................................. 16

Water quality.................................................................................................. 16

Selected references........................................................................................ 18

Appendixes................................................................................................... 21

Explanation of codes and abbreviations contained in data tables in the appendixes......................................................................................... 23

Appendix A-Ground-water data ....................................................................

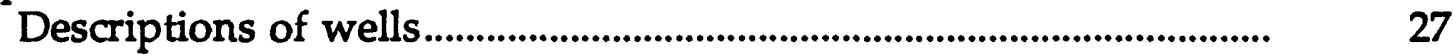

Hydrographs ..................................................................................................... 33

Appendix B--Surface-water data.................................................................. 103

Descriptions of sites............................................................................. 105

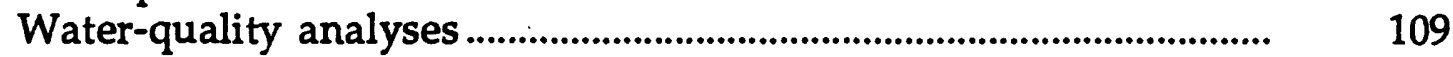




\section{ILLUSTRATIONS}

Page

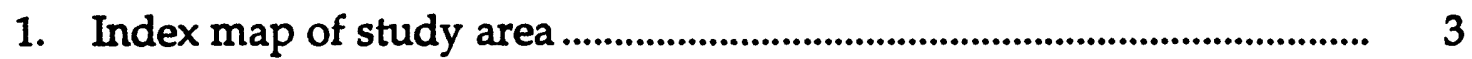

Figures 2-10. Maps showing:

2. Location of hydrologic monitoring sites in the Divide Section........ 4

3. Location of hydrologic monitoring sites in the vicinity of Pools $D$ and $\mathrm{E}$.

4. Location of hydrologic monitoring sites in the vicinity of Pool C... 6

5. Location of hydrologic monitoring sites in the vicinity of Pool B... 7

6. Location of hydrologic monitoring sites in the vicinity of Pool A... 8

7. Location of hydrologic monitoring sites in the vicinity of

Aberdeen Pool

8. Location of hydrologic monitoring sites in the vicinity of

Columbus Pool.

9. Location of hydrologic monitoring sites in the vicinity of Aliceville Pool.

10. Location of hydrologic monitoring sites in the vicinity of Gainesville Pool. 


\section{CONVERSION FACTORS AND VERTICAL DATUM}

Multiply

inch (in.)

foot $(\mathrm{ft})$

mile (mi)

square mile $\left(\mathrm{mi}^{2}\right)$

acre-foot (acre-ft)

cubic foot per second $\left(\mathrm{ft}^{3} / \mathrm{s}\right)$

gallon per minute (gal/min)

micromho per centimeter

at $25^{\circ} \mathrm{Celsius}$ (umho/cm at $25^{\circ} \mathrm{C}$ )
By

25.40

0.3048

1.609

2.590

1,233

0.02832

0.06308

1.000
To obtain

millimeter

meter

kilometer

square kilometer

cubic meter

cubic meter per second

liter per second

microsiemens per centimeter at $25^{\circ}$ Celsius

To convert degrees Celsius $\left({ }^{\circ} \mathrm{C}\right)$ to Fahrenheit $\left({ }^{\circ} \mathrm{F}\right)$, use the following:

$$
{ }^{\circ} \mathrm{F}=9 / 5^{\circ} \mathrm{C}+32
$$

Sea level: In this report "sea level" refers to the National Geodetic Vertical Datum of 1929 (NGVD of 1929)-a geodetic datum derived from a general adjustment of the first-order level nets of both the United States and Canada, formerly called "Sea Level Datum of 1929." 


\title{
HYDROLOGIC MONITORING IN THE AREA OF THE TENNESSEE-TOMBIGBEE WATERWAY, MISSISSIPPI- ALABAMA, FISCAL YEAR 1989
}

by Fred Morris III

\begin{abstract}
This report, the sixteenth in a series of annual reports, presents hydrologic data collected in the area of the Tennessee-Tombigbee Waterway during the fiscal year ending September 30, 1989. Included in this report are data on ground-water levels and surface-water stage, discharge, and quality. These data were obtained at the request of the U.S. Army Corps of Engineers, Mobile District, as part of a comprehensive program to monitor the hydrologic effects of construction and operation of the Waterway.
\end{abstract}




\section{PROGRAM CHANGES FOR 1989}

For Fiscal Year 1989 (FY89), the disposal area network was discontinued and the number of sites in the surface-water network at which water-quality data were collected was decreased from 26 to 15 . Beginning in FY89, waterquality samples were analyzed by the U.S. Geological Survey (USGS).

\section{HYDROLOGICMONITORING}

Surface-water sites and observation wells in the original hydrologic monitoring network, used to define hydrologic conditions in the area of the Tennessee-Tombigbee Waterway prior to construction, are described by Brahana and others (1974) in the U.S. Army Corps of Engineers report, "First Supplemental Environmental Report, Continuing Environmental Studies, Tennessee-Tombigbee Waterway, Alabama and Mississippi." The present hydrologic monitoring network includes:

- Major aquifers that may have been stressed by the Waterway construction and operation;

- Surface-water sites near locks and dams where the effects of construction may have been greatest, or at sites of inflow or outflow;

- Areas of known or suspected hydrologic problems;

- Selected sites on and near Pickwick Lake and Demopolis Lake.

The purpose of the present hydrologic monitoring network is to document changes in the hydrologic environment that may occur during operation of the Waterway. The locations of all the hydrologic monitoring sites in the vicinity of the Waterway at which data have been collected since the beginning of the project are shown in figures 1 through 10, except for a few sites which are not shown because of the limited coverage of the maps (furnished by the U.S. Army Corps of Engineers, Mobile District). 


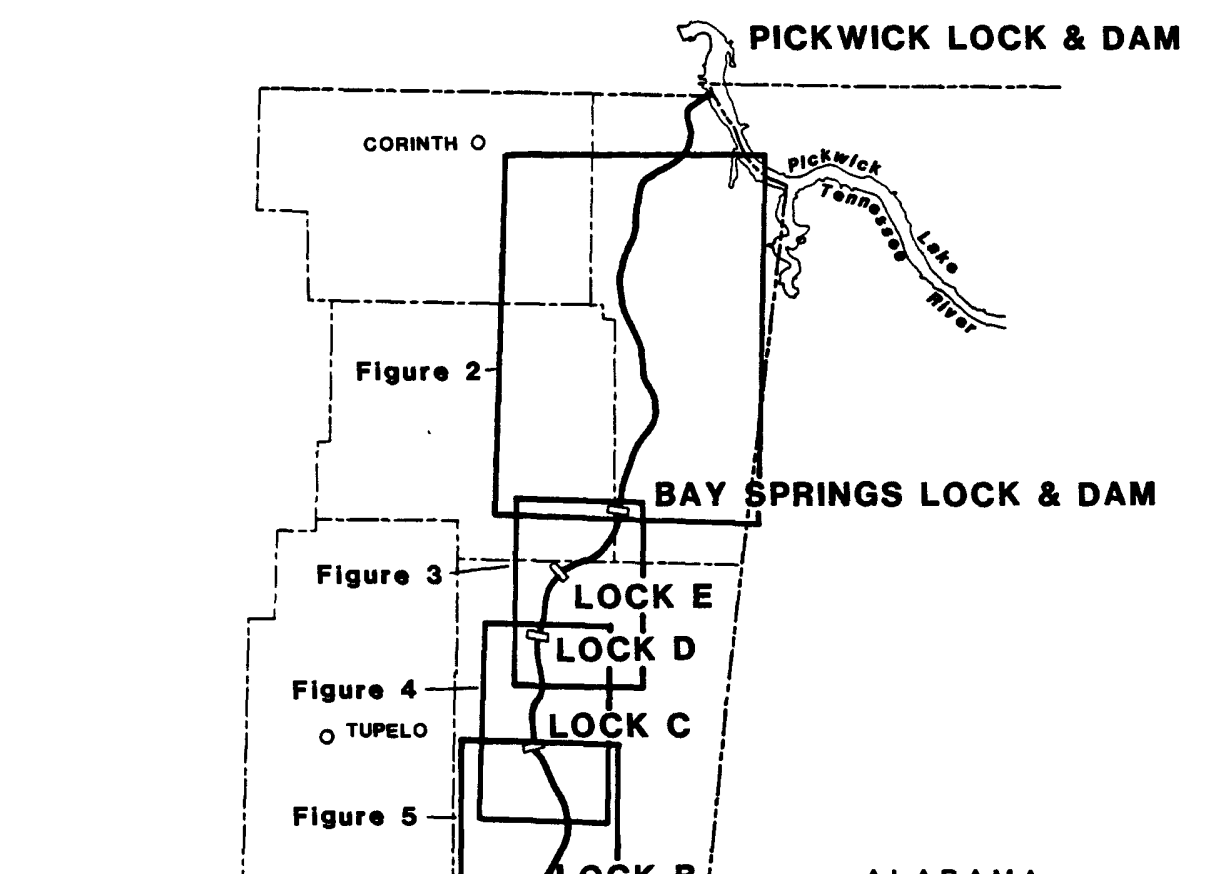

Figure 8

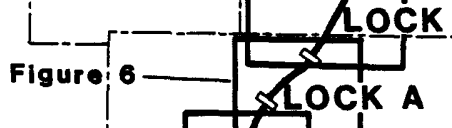

ALABAMA

$\rightarrow$

Figure
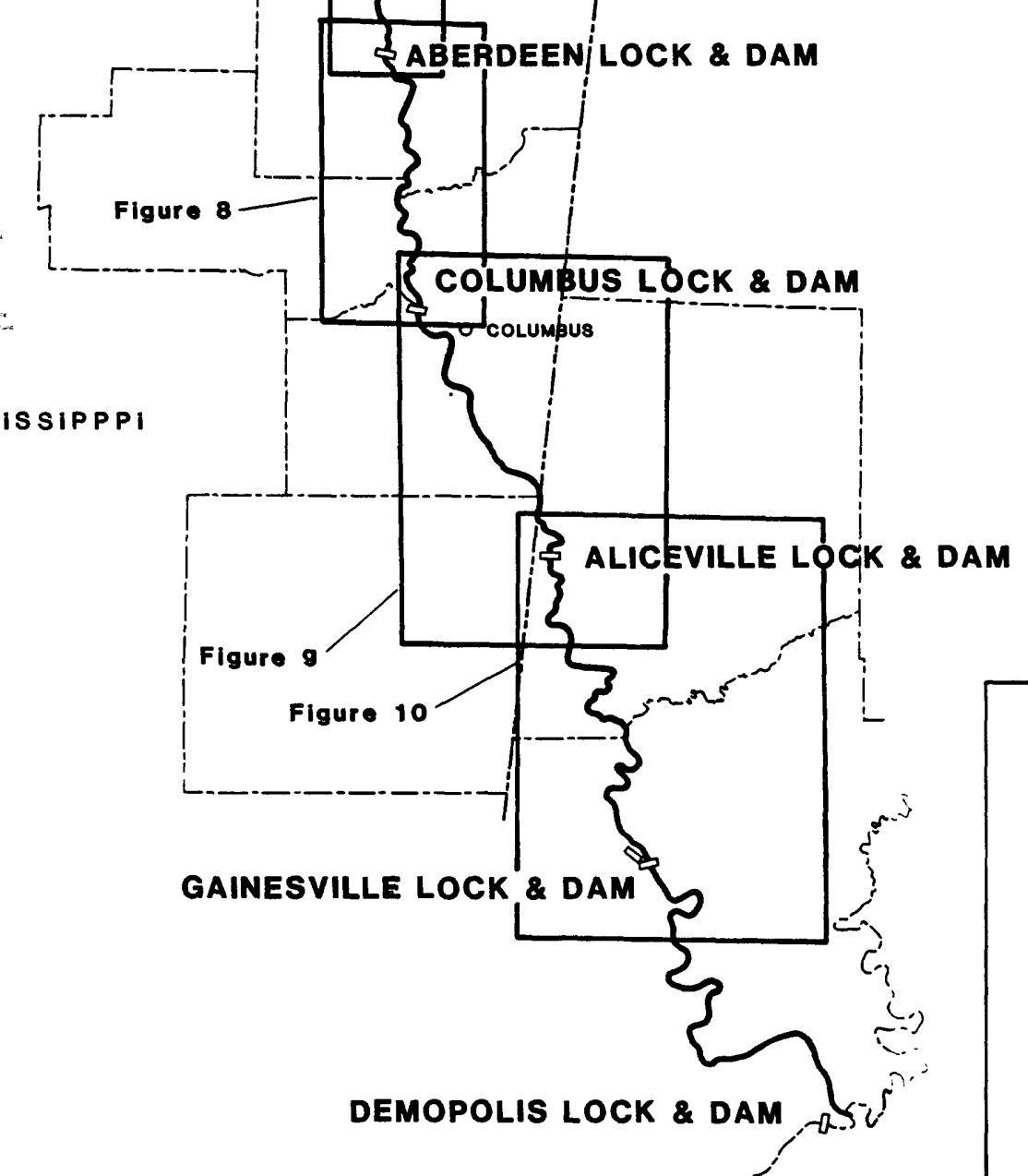

TENNESSEE-TOMBIGBEE WATERWAY HYDROLOGIC MONITORING PROGRAM

\section{INDEX MAP}

PREPARED FOR

MOBILE DISTRICT, CORPS OF ENGINEERS MOBILE, ALABAMA

$$
\text { BY }
$$




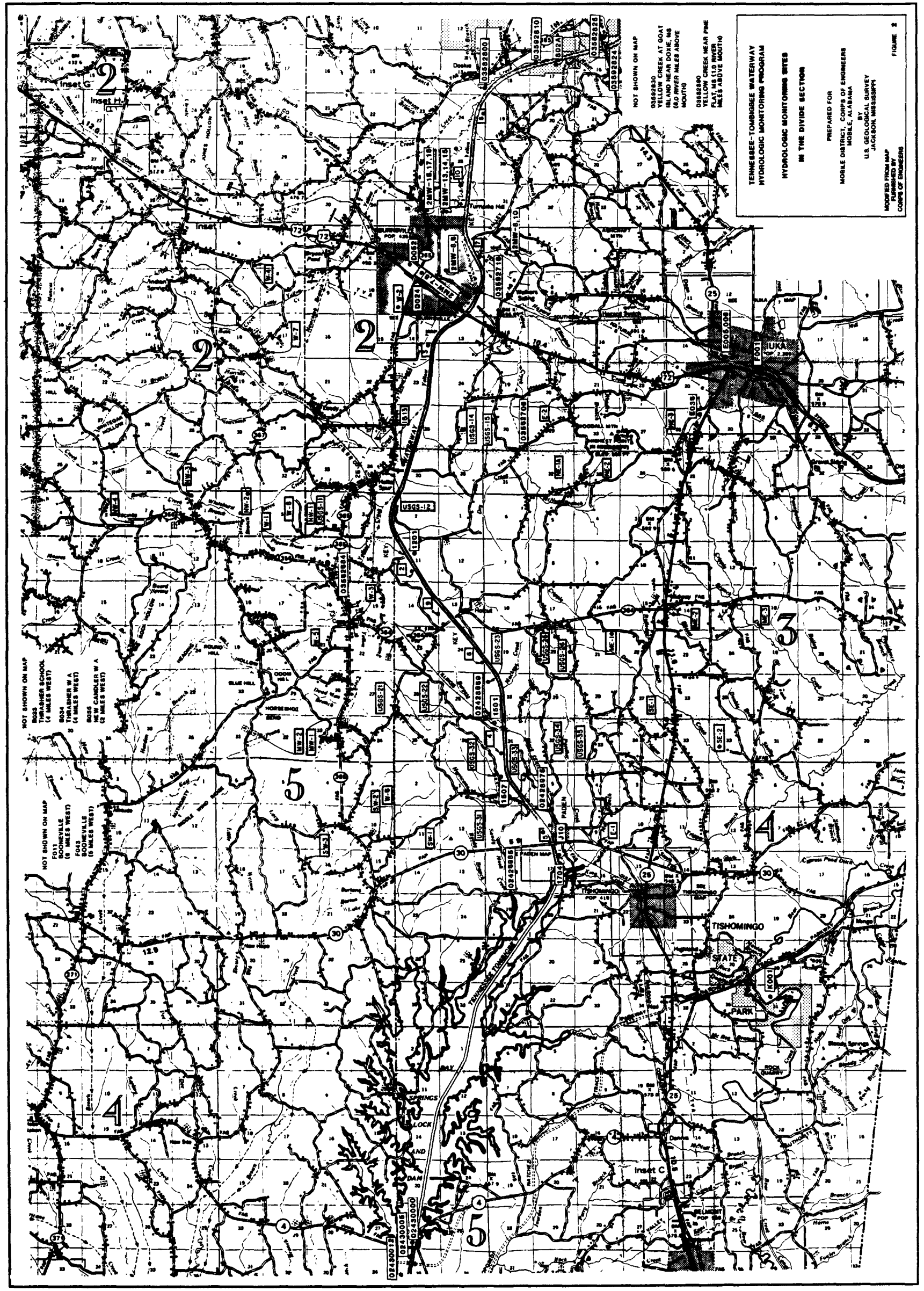




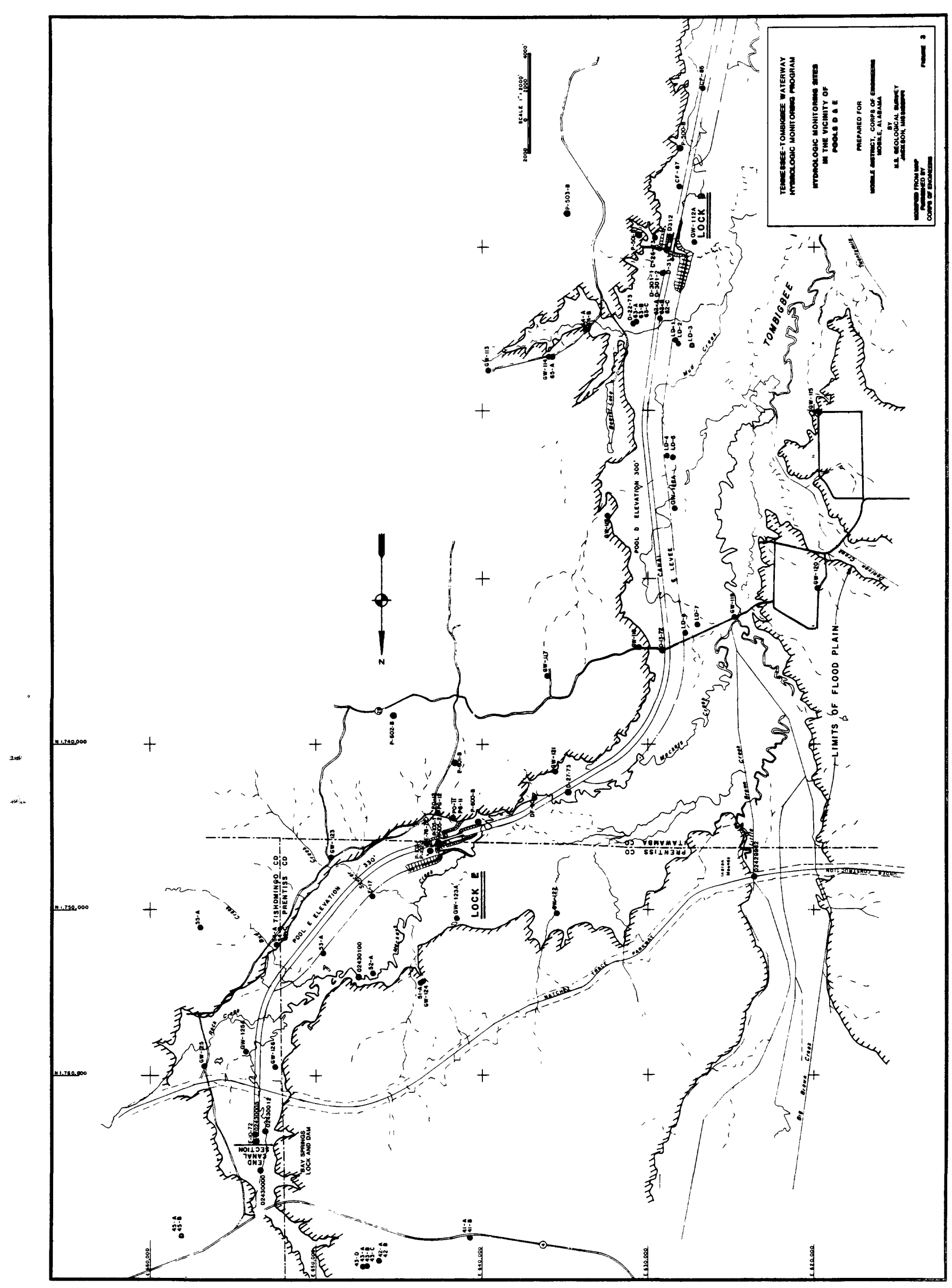




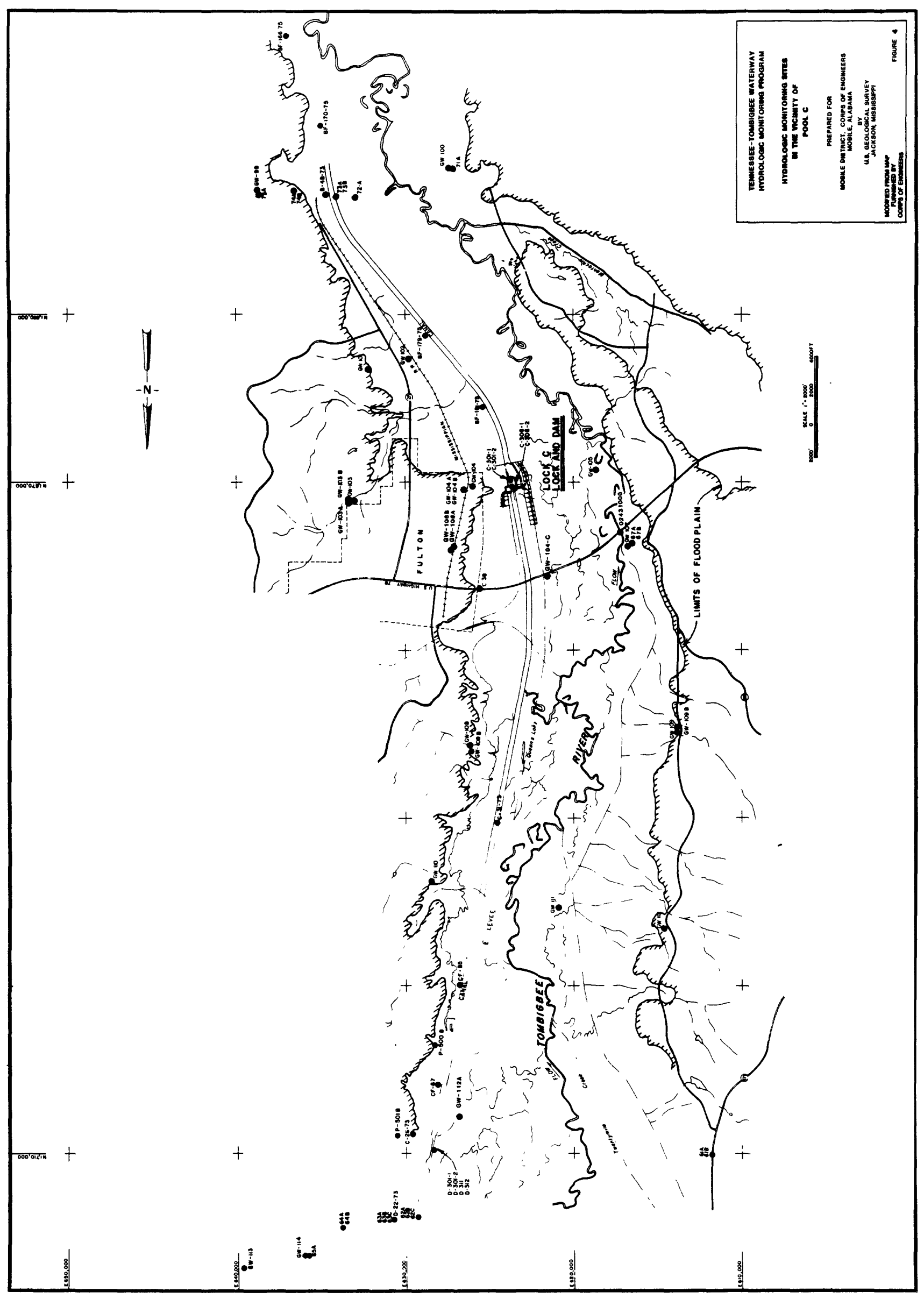




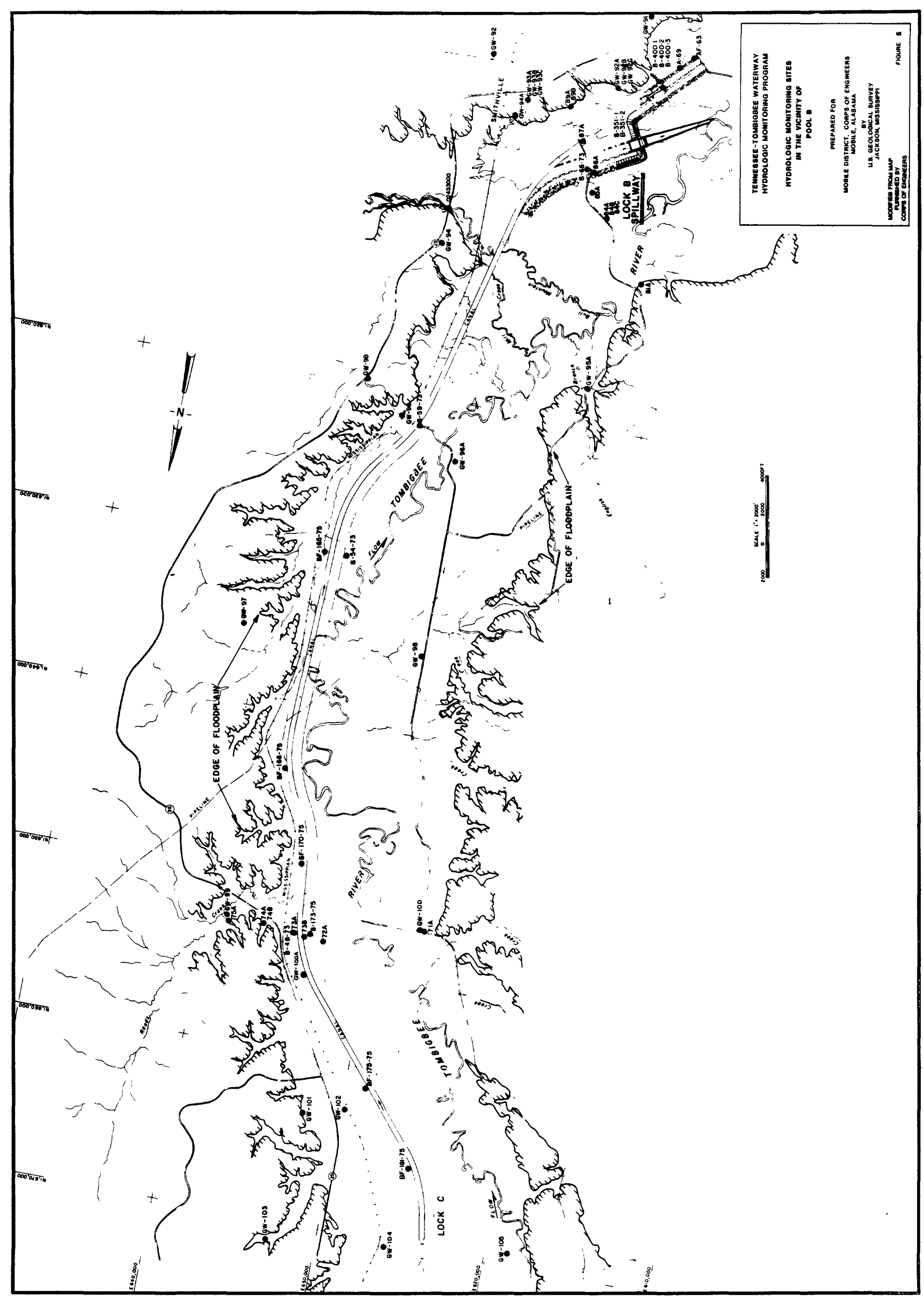




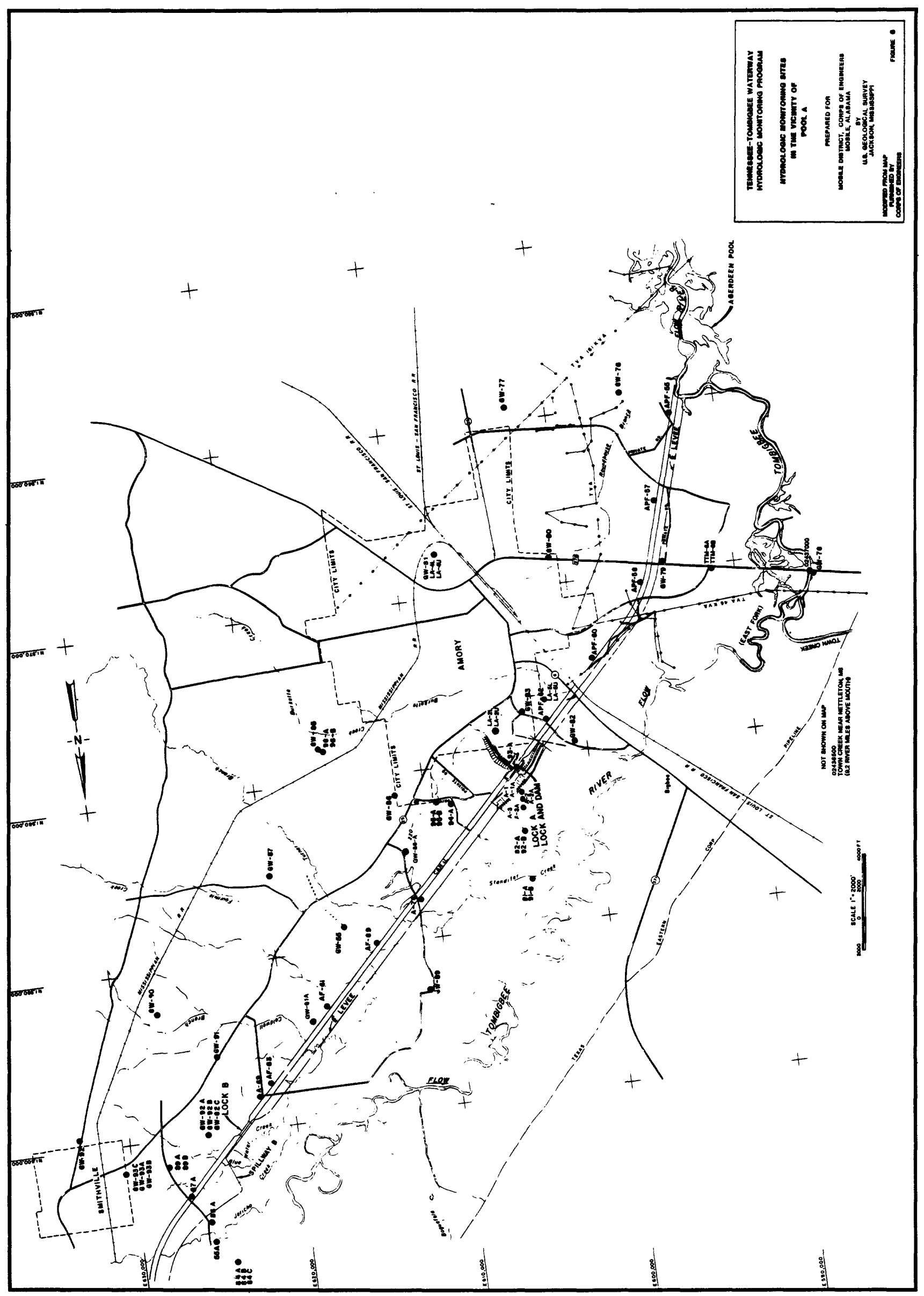




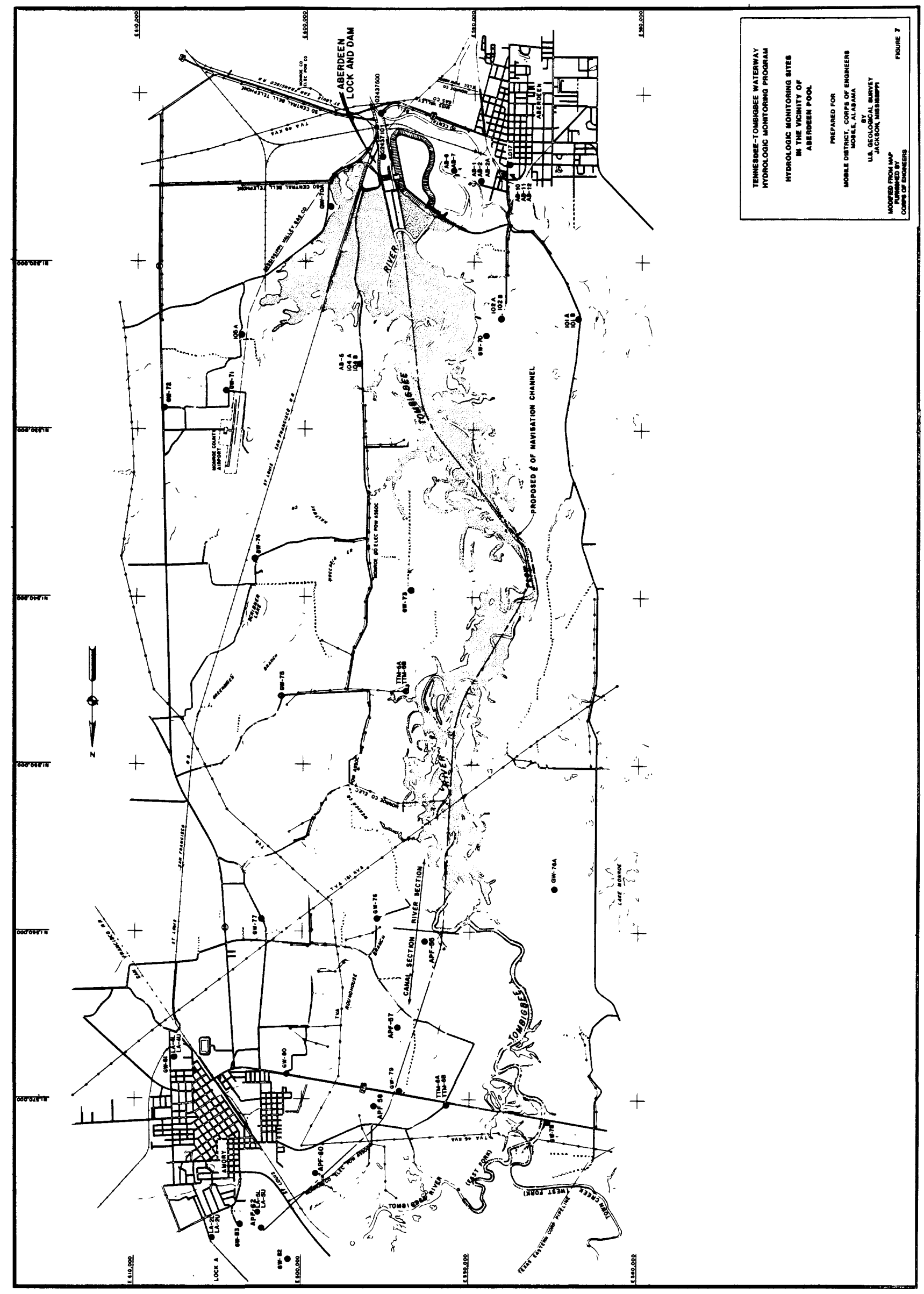




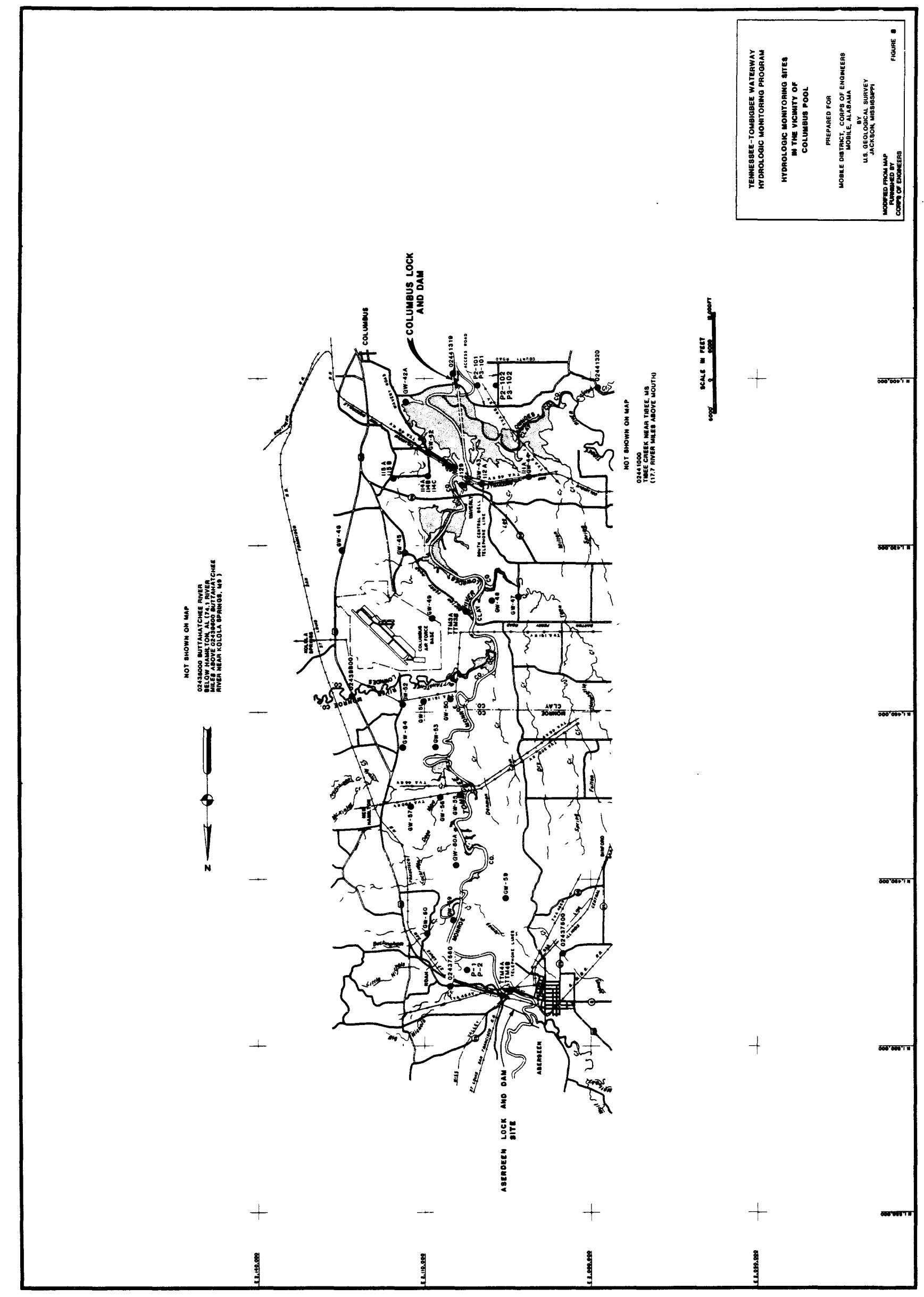




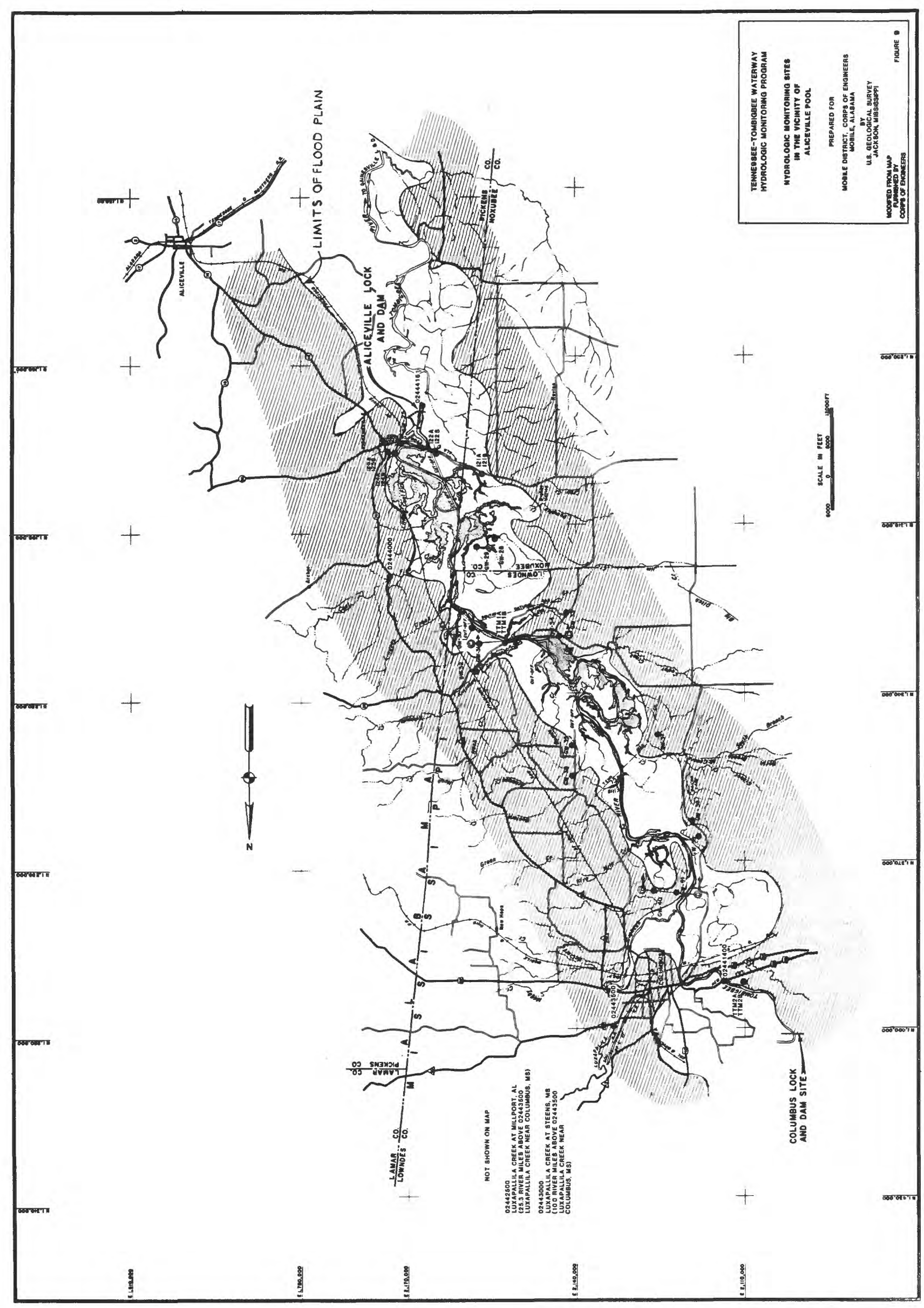




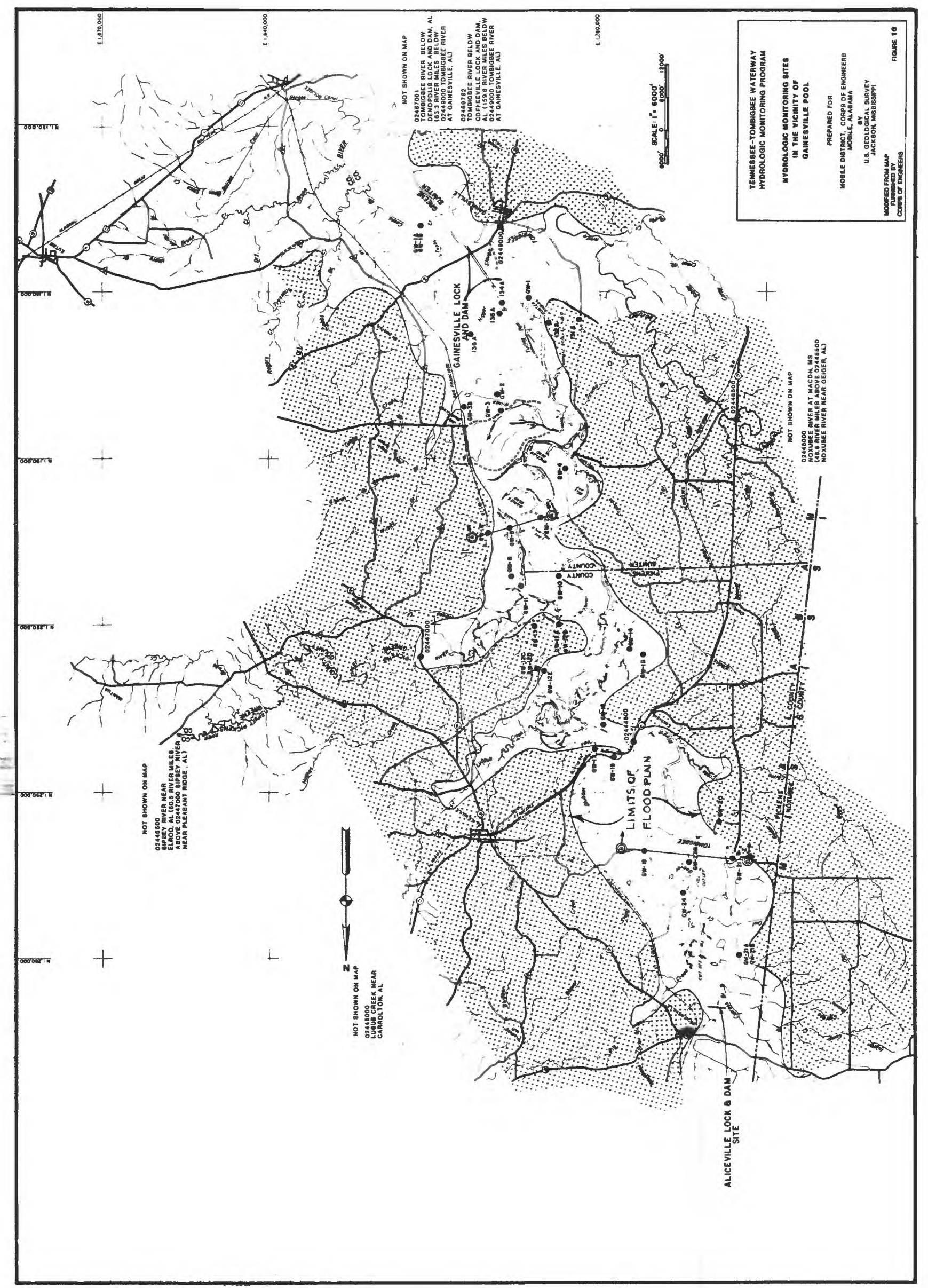




\section{Ground Water}

Network

The present ground-water network consists of 267 wells in the regional aquifers and the shallower alluvial and terrace aquifers. The relation between shallow water-bearing units and regional aquifers is described by Brahana and others (1974). The descriptions of wells in the network are tabulated in Appendix A.

\section{Levels}

Under natural conditions, water levels in wells fluctuate seasonally and reflect recharge to and discharge from aquifers. Water-level fluctuations ranging from less than 1 foot to more than 10 feet per year have been observed in the aquifers in the study area. Water-level fluctuations generally were larger in the alluvial and terrace aquifers than in the regional aquifers.

During FY89, water levels in 134 observation wells in the network were scheduled to be measured quarterly, 27 wells during the first and second quarters, and 106 during the first quarter only, by the USGS. However, 16 wells (11C, 14A, 14C, 23D, 23I, 23L, 23O, 42A, 43A, 43D, 45A, GW94, GW97, MW2-3, W8-1, and 6DP164) were dry or could not be measured because of obstructions in the wells. Hydrographs showing water-level variations in the wells for the period of USGS record are presented in Appendix A.

\section{Surface Water}

\section{Network}

The surface-water network, which is designed to monitor water quality, currently consists of 15 sites in the area of the Tennessee-Tombigbee Waterway. Descriptions of these sites are tabulated in Appendix B.

\section{Stage and Discharge}

Surface-water stage and discharge data were collected at numerous sites (including most sites at which water-quality data were collected) in the area of the Tennessee-Tombigbee Waterway. The collection of stage and discharge data at these sites was funded by cooperative programs with various State and Federal agencies. Data collected at these sites are available either in the Jackson, Miss., or Tuscaloosa, Ala., office of the USGS. 


\section{Quality}

Water-quality data were collected by the USGS at 15 surface-water sites in the network during the 1989 reporting period (Appendix B). Five sites were added at the beginning of the 1989 reporting period; 16 sites were discontinued.

New sites:

$02433000 \quad$ Bull Mountain Creek near Smithville, Miss.

02441400

02446500

Tombigbee River near Columbus, Miss.

02448500

Sipsey River near Elrod, Ala.

02467001

Noxubee River near Geiger, Ala.

Tombigbee River below Demopolis Lock and Dam, Ala.

Discontinued sites:

$\begin{array}{ll}343140088192235 & \text { TTW Bay Springs Lake Navigation Mile 412.3 } \\ 02430005 & \text { Tenn-Tom Waterway below Bay Springs Lock and Dam, Miss. } \\ 342201088242935 & \text { TTW Lock "D" Pool Sedimentation Range 1AD } \\ 340103088285435 & \text { TTW Lock "A" Pool Sedimentation Range 1AA } \\ 02430100 & \text { Mackeys Creek near Moores Mill, Miss. } \\ 02437000 & \text { Tombigbee River near Amory, Miss. } \\ 335008088311335 & \text { TTW Aberdeen Lake Sedimentation Range 1A } \\ 02437101 & \text { Tombigbee River below Aberdeen Lock and Dam, Miss. } \\ 334219088281935 & \text { TTW Columbus Lake McKinley Creek Bend SR 50A } \\ 333119088291435 & \text { TTW Columbus Lake Sedimentation Range 1A } \\ 02441391 & \text { Tombigbee River below Columbus Lock and Dam, Miss. } \\ 02444158 & \text { Tombigbee River above Bevill Lock and Dam, Ala. } \\ 02444161 & \text { Tombigbee River below Bevill Lock and Dam, Ala. } \\ 02447020 & \text { Tombigbee River above Gainesville Lock and Dam, Ala. } \\ 02449000 & \text { Tombigbee River at Gainesville, Ala. } \\ 02466998 & \text { Tombigbee River above Demopolis Lock and Dam, Ala. }\end{array}$

Data for site 02433000, Bull Mountain Creek near Smithville, Miss., and site 02441000 , Tibbee Creek near Tibbee, Miss. may not represent water-quality conditions upstream of the sampling sites. Normal pool elevation of 245.00 feet above sea level for Pool B creates a stage of about 10.2 feet at the Bull Mountain Creek site; a normal pool elevation of 163.00 feet above sea level for Columbus Lake creates a stage of about 8.8 feet at the Tibbee Creek site, resulting in variable backwater conditions. Measurements of stream discharge were not obtained during backwater conditions. 
Data for suspended-sediment concentration, particle-size distribution of suspended sediment, and particle-size distribution of stream bed material were collected at site 02436500 , Town Creek near Nettleton, Miss. and site 02448000, Noxubee River at Macon, Miss. Data for suspended-sediment concentration and particle-size distribution of stream bed material were collected at the following sites:

$\begin{array}{ll}332929088273300 & \text { TTW Aliceville Lake above Columbus Bend } \\ \mathbf{0 2 4 4 1 4 9 8} & \text { Tombigbee River Columbus Bend SR 11B at Columbus, Miss. } \\ 332751088261000 & \text { TTW Aliceville Lake Columbus Cut } \\ 3321120882235 & \text { TTW Aliceville Lake above Hairston Bend } \\ 3321000882248 & \text { TTW Aliceville Lake Hairston Bend SR 11HB } \\ 02447008 & \text { Tombigbee River above Cooks Bend SR 5A near Warsaw, Ala. } \\ \mathbf{0 2 4 4 7 0 1 0} & \text { Tombigbee River Cooks Bend near Warsaw, Ala. } \\ 325645088100700 & \text { TTW Gainesville Lake Cooks Bend Cut SR 4AD } \\ 323704087542400 & \text { TTW Demopolis Lake above Rattlesnake Bend SR 12CA } \\ 323653087540800 & \text { TTW Demopolis Lake Rattlesnake Bend SR RB1 } \\ 323642087541800 & \text { TTW Demopolis Lake Rattlesnake Cut SR 12CB. }\end{array}$

Data for suspended-sediment concentration was collected at the following sites:

$\begin{array}{ll}02430500 & \text { Tombigbee River near Marietta, Miss. } \\ 02430680 & \text { Twentymile Creek near Guntown, Miss. } \\ 02430690 & \text { Twentymile Creek near Mantachie, Miss. } \\ 02431000 & \text { Tombigbee River near Fulton, Miss. } \\ 02431410 & \text { Mantachie Creek below Dorsey, Miss. } \\ 02433500 & \text { Tombigbee River at Bigbee, Miss. } \\ 02437000 & \text { Tombigbee River near Amory, Miss. } \\ 02437500 & \text { Tombigbee River at Aberdeen, Miss. } \\ 02439400 & \text { Buttahatchee River near Aberdeen, Miss. } \\ 02441400 & \text { Tombigbee River near Columbus, Miss. } \\ 02443500 & \text { Luxapallila Creek near Columbus, Miss. } \\ 3320300882122 & \text { TTW Aliceville Lake Hairston Cut } \\ 02444157 & \text { Tombigbee River at St Hwy 86 near Pickensville, Ala. } \\ 02444500 & \text { Tombigbee River near Cochrane, Ala. }\end{array}$

Specific conductance and water temperature were measured daily, and miscellaneous samples were collected periodically at two sites on the lower Tombigbee River: site 02449000, Tombigbee River at Gainesville, Ala. (monthly), and site 02469762, Tombigbee River below Coffeeville Lock and Dam, Ala. (quarterly). The results of these USGS measurements and analyses are presented in Appendix B. Collection of these data was funded by cooperative agreements with various State and Federal agencies. 


\section{QUALITY ASSURANCE}

\section{Ground-Water Levels}

The collection, analysis, and computation of ground-water level records are conducted in accordance with techniques and procedures established by the USGS and are within the guidelines recommended in the "National Handbook of Recommended Methods for Water-Data Acquisition" (Office of Water Data Coordination, 1977).

\section{Surface-Water Stage and Discharge}

The collection, analysis, and computation of surface-water stage and discharge records are conducted in accordance with procedures described in a series entitled "Techniques of Water-Resources Investigations of the U.S. Geological Survey" (TWRI). Field activities are presented in three chapters entitled "General Procedure for Gaging Streams" (Carter and Davidian, 1968); "Stage Measurements at Gaging Stations" (Buchanan and Somers, 1968); and "Discharge Measurements at Gaging Stations" (Buchanan and Somers, 1969); and more recently in Water-Supply Paper 2175, "Measurement and Computation of Streamflow: Volume 1, Measurement of Stage and Discharge" (Rantz and others, 1982). Daily discharge is computed in conformance with procedures described in Water Supply Paper 2175, "Measurement and Computation of Streamflow: Volume 2, Computation of Discharge" (Rantz and others, 1982). All these procedures are within the guidelines recommended in the "National Handbook of Recommended Methods for Water-Data Acquisition" (Office of Water Data Coordination, 1977).

\section{Water Quality}

Procedures used by the USGS in the collection and analysis of samples of water and bottom materials are in conformance with the methods of laboratory analysis and sample preservation and handling described in TWRI Chapter A1, Book 5, "Methods for Determination of Inorganic Substances in Water and Fluvial Sediments" (Fishman and Friedman, 1989).

Water samples collected as a part of this investigations were analyzed in one of the National Water-Quality Laboratories of the Water Resources Division, USGS. The National Water Quality Laboratories have an effective quality control program that includes the use of duplicate samples and standard reference water samples. TWRI "Quality Assurance Practices for the Chemical and Biological Analysis of Water and Fluvial Sediments" 
(Friedman and Erdmann, 1982) describes quality control techniques, quality assurance practices, and statistical techniques used by the National WaterQuality Laboratories.

The methods used in the collection and analyses of bacteriological samples are given in TWRI "Methods for collection and Analysis of Aquatic Biological and Microbiological Samples" (Britton and Greeson, 1989). All bacteriological analyses were performed in the field within a few hours after the samples were collected.

The methods used in the collection and analyses of suspended-sediment samples are given in TWRI "Field Methods for Measurement of Fluvial Sediment" (Guy and Norman, 1970) and "Laboratory Theory and Methods for Sediment Analysis" (Guy, 1969).

Procedures used for water-quality field data collection are in accordance with techniques established by the USGS and are within the guidelines recommended in the "National Handbook of Recommended Methods for Water-Data Acquisition" (Office of Water Data Coordination, 1977). 


\section{SELECTED REFERENCES}

Brahana, J.V., German, E.R., Davis, M.E., and Wasson, B.E., 1974, Predicted effects of the Tennessee-Tombigbee Waterway on the hydrologic environment, Mississippi-Alabama in First supplemental environmental report, continuing environmental studies, Tennessee-Tombigbee Waterway, Alabama and Mississippi: U.S. Army Corps of Engineers, Mobile District, v. 2, 3, and 4.

Britton, L.J., and Greeson, P.E., editors, 1989, Methods for collection and analysis of aquatic biological and microbiological samples, Chapter A4 of Book 5, Laboratory analyses: U.S. Geological Survey Techniques of Water-Resources Investigations, $363 \mathrm{p}$.

Buchanan, T.J., and Somers, W.P., 1968, Stage measurements at gaging stations, Chapter A7 of Book 3, Applications of hydraulics: U.S. Geological Survey Techniques of Water-Resources Investigations, $28 \mathrm{p}$.

----1969, Discharge measurements at gaging stations, Chapter A8 of Book 3, Applications of hydraulics: U.S. Geological Survey Techniques of WaterResources Investigations, $65 \mathrm{p}$.

Carter, R.W., Davidian, Jacob, 1968, General procedure for gaging streams, Chapter A6 of Book 3, Applications of hydraulics: U.S. Geological Survey Techniques of Water-Resources Investigations, $13 \mathrm{p}$.

Fishman, M.J., and Friedman, L.C., 1989, Methods for determination of inorganic substances in water and fluvial sediments, Chapter A1 of Book 5, Laboratory analysis: U.S. Geological Survey Techniques of Water-Resources Investigations, $545 \mathrm{p}$.

Friedman, L.C., and Erdmann, D.E., 1982, Quality assurance practices for the chemical and biological analyses of water and fluvial sediments, Chapter A6 of Book 5, Laboratory analysis: U.S. Geological Survey Techniques of Water-Resources Investigations, $181 \mathrm{p}$.

Guy, H.P., 1969, Laboratory theory and methods for sediment analysis, Chapter C1 of Book 5, Laboratory analysis: U.S. Geological Survey Techniques of WaterResources Investigations, $58 \mathrm{p}$.

Guy, H.P., and Norman, V.W., 1970, Field methods for measurement of fluvial sediment, Chapter C2 of Book 3, Application of hydraulics: U.S. Geological Survey Techniques of Water-Resources Investigations, $59 \mathrm{p}$.

Leake, S.A., 1977, Simulation of flow to a partially penetrating trench: U.S. Geological Survey Journal of Research, v. 5, no. 5, 15 p. 
Morris, Fred, III, 1983, Hydrologic monitoring in the area of the TennesseeTombigbee Waterway, Mississippi-Alabama, Fiscal Year 1982: U.S. Geological Survey Open-File Report 83-764, 189 p.

---1984, Hydrologic monitoring in the area of the Tennessee-Tombigbee Waterway, Mississippi-Alabama, Fiscal Year 1983: U.S. Geological Survey Open-File Report 84-717, $174 \mathrm{p}$.

---1985, Hydrologic monitoring in the area of the Tennessee-Tombigbee Waterway, Mississippi-Alabama, Fiscal Year 1984: U.S. Geological Survey Open-File Report 85-692, 152 p.

--1986, Hydrologic monitoring in the area of the Tennessee-Tombigbee Waterway, Mississippi-Alabama, Fiscal Year 1985: U.S. Geological Survey Open-File Report 86-493, 311 p.

-1-1988, Hydrologic monitoring in the area of the Tennessee-Tombigbee Waterway, Mississippi-Alabama, Fiscal Year 1986: U.S. Geological Survey Open-File Report 88-321, 500 p.

--1991, Hydrologic monitoring in the area of the Tennessee-Tombigbee Waterway, Mississippi-Alabama, Fiscal Year 1987: U.S. Geological Survey Open-File Report 91-447, 245 p.

--1991, Hydrologic monitoring in the area of the Tennessee-Tombigbee Waterway, Mississippi-Alabama, Fiscal Year 1988: U.S. Geological Survey Open-File Report 91-511, $231 \mathrm{p}$.

Office of Water Data Coordination, U.S. Geological Survey, 1977: National handbook of recommended methods for water-data acquisition.

Rantz, S.E., and others, 1982, Measurement and computation of streamflow: U.S. Geological Survey Water-Supply Paper 2175, v. 1 and 2, 631 p.

Seaber, P.R., Kapinos, F.P., and Knapp, G.L., 1987, Hydrologic unit maps: U.S. Geological Survey Water-Supply Paper 2294, 63 p.

Shell, J.D., 1976, Hydrologic monitoring in the area of the Tennessee-Tombigbee Waterway, Mississippi-Alabama, July 1974 through June 1975: U.S. Geological Survey open-file report, $252 \mathrm{p}$.

---1977, Hydrologic monitoring in the area of the Tennessee-Tombigbee Waterway, Mississippi-Alabama, July 1975 through September 1976: U.S. Geological Survey open-file report, $227 \mathrm{p}$. 
Shell, J.D., 1978, Hydrologic monitoring in the area of the Tennessee-Tombigbee Waterway, Mississippi-Alabama, Fiscal Year 1977: U.S. Geological Survey openfile report, $155 \mathrm{p}$.

--1979, Hydrologic monitoring in the area of the Tennessee-Tombigbee Waterway, Mississippi-Alabama, Fiscal Year 1978: U.S. Geological Survey open-file report, $198 \mathrm{p}$.

--1980, Hydrologic monitoring in the area of the Tennessee-Tombigbee Waterway, Mississippi-Alabama, Fiscal Year 1979: U.S. Geological Survey open-file report, $153 \mathrm{p}$.

Tomaszewski, D.J., 1981, Hydrologic monitoring in the area of the TennesseeTombigbee Waterway, Mississippi-Alabama, Fiscal Year 1980: U.S. Geological Survey Open-File Report 81-546, 142 p.

--1982, Hydrologic monitoring in the area of the Tennessee-Tombigbee Waterway, Mississippi-Alabama, Fiscal Year 1981: U.S. Geological Survey Open-File Report 82-503, 140 p. 


\section{APPENDIXES}

21

PAGE 23 FOLLOWS 
EXPLANATION OF CODES AND ABBREVIATIONS CONTAINED IN DATA TABLES

IN THE APPENDIXES

PRINCIPAL AQUIFER

Geologic

unit code

Aquifer name and age

110ALVM

11OTRCS

211TBGB

Quaternary alluvium, Quaternary
Undifferentlated terrace deposits, Quaternary

211EUTH

Tomblgbee Sand Member of Eutaw Eormation, Upper Cretaceous

Eutaw Formation, Upper Cratacsous

211EUTWR Eutaw Formation (Restricted), Upper Cretaceous

211MCSN

wer Eut aw Formation, Upper Cretaceous

211GORD

McShan Formation, Upper Cretaceous

Gordo Formation. upper Cretaceous

Mississippian system, Mississippian

HYDROLOGIC UNIT

An eight-digit hydrologic unit code refers to a specific drainage basin

as delineated by the office of Water Data Coordination on the state

Hydrologic Unit Maps (Seaber and others, 1987).

\section{WATER-QUALITY REMARKS}

Remark Code

Remark

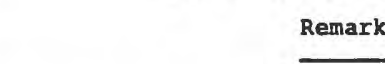

Estimated value

$\mathbf{E}$

Actual value is known to be less than the value shown

Results based on colony count outside the acceptance

range (non-1deal colony count) 
APPENDIX A

GROUND-WATER DATA

25

PAGE 27 FOLLOW'S 


\section{APPENDIX A}

GROUND-WATER DATA

DESCRIPTIONS OF WELLS

27

PAGE 29 FOLLOWS 


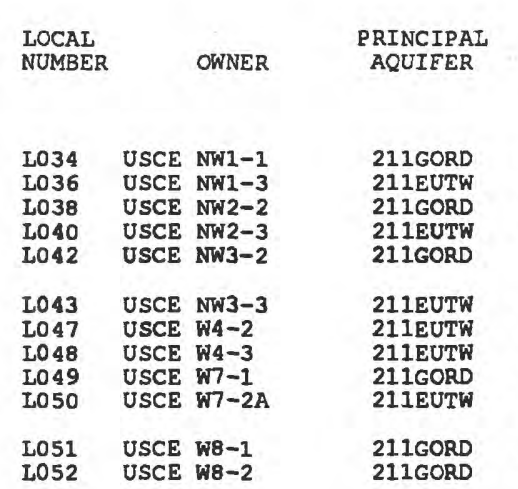

\begin{tabular}{|c|c|c|}
\hline $\begin{array}{l}\text { A023 } \\
\text { A024 } \\
\text { A025 } \\
\text { B005 } \\
\text { B008 }\end{array}$ & $\begin{array}{l}\text { USCE } \\
\text { USCE } \\
\text { USCE } \\
\text { USCE } \\
\text { USCE }\end{array}$ & $\begin{array}{l}\text { GW118 } \\
\text { GW119 } \\
\text { GW120 } \\
\text { GW117 } \\
\text { P601B }\end{array}$ \\
\hline $\begin{array}{l}\text { Bo } 09 \\
\text { D039 } \\
\text { D040 } \\
\text { DO } 41 \\
\text { D0 42 }\end{array}$ & $\begin{array}{l}\text { USCE } \\
\text { USCE } \\
\text { USCE } \\
\text { USCE } \\
\text { USCE }\end{array}$ & $\begin{array}{l}\text { P602B } \\
\text { CF87 } \\
\text { GW110 } \\
\text { GW112A } \\
\text { GW115 }\end{array}$ \\
\hline $\begin{array}{l}\text { D0 } 43 \\
\text { D0 44 } \\
\text { D045 } \\
\text { D046 } \\
\text { E005 }\end{array}$ & $\begin{array}{l}\text { USCE } \\
\text { USCE } \\
\text { USCE } \\
\text { USCE } \\
\text { USCE }\end{array}$ & $\begin{array}{l}\text { GW116 } \\
\text { GW116A } \\
\text { P500B } \\
\text { P501B } \\
65 A\end{array}$ \\
\hline $\begin{array}{l}\text { E009 } \\
\text { E010 } \\
\text { E011 } \\
\text { G065 } \\
\text { G066 }\end{array}$ & $\begin{array}{l}\text { USCE } \\
\text { USCE } \\
\text { USCE } \\
\text { USCE } \\
\text { USCE }\end{array}$ & $\begin{array}{l}\text { GW113 } \\
\text { GW114 } \\
\text { P503B } \\
67 A \\
67 B\end{array}$ \\
\hline $\begin{array}{l}\text { G067 } \\
\text { G068 } \\
\text { G070 } \\
\text { G072 } \\
\text { G074 }\end{array}$ & $\begin{array}{l}\text { USCE } \\
\text { USCE } \\
\text { USCE } \\
\text { USCE } \\
\text { USCE }\end{array}$ & $\begin{array}{l}\text { GW1068 } \\
\text { GW106A } \\
\text { GW104C } \\
\text { C38 } \\
\text { GW104A }\end{array}$ \\
\hline $\begin{array}{l}\text { G075 } \\
\text { G076 } \\
\text { G077 } \\
\text { G078 } \\
\text { G079 }\end{array}$ & $\begin{array}{l}\text { USCE } \\
\text { USCE } \\
\text { USCE } \\
\text { USCE } \\
\text { USCE }\end{array}$ & $\begin{array}{l}\text { GW104B } \\
\text { GW105 } \\
\text { GW107 } \\
\text { GW108 } \\
\text { GW108B }\end{array}$ \\
\hline $\begin{array}{l}\text { G080 } \\
\text { G081 } \\
\text { G082 } \\
\text { G083 } \\
\text { G084 }\end{array}$ & $\begin{array}{l}\text { USCE } \\
\text { USCE } \\
\text { USCE } \\
\text { USCE } \\
\text { USCE }\end{array}$ & $\begin{array}{l}\text { GW109 } \\
\text { GW109B } \\
\text { GWI11 } \\
\text { GW112 } \\
\text { GW104 }\end{array}$ \\
\hline $\begin{array}{l}\text { K039 } \\
\text { K0 41 } \\
\text { K042 } \\
\text { L014 } \\
\text { L016 }\end{array}$ & $\begin{array}{l}\text { USCE } \\
\text { USCE } \\
\text { USCE } \\
\text { USCE } \\
\text { USCE }\end{array}$ & $\begin{array}{l}71 A \\
\text { GW100 } \\
\text { BF179-75 } \\
74 A \\
75 A\end{array}$ \\
\hline $\begin{array}{l}\text { L017 } \\
\text { L021 } \\
\text { LO22 } \\
\text { L023 } \\
\text { L024 }\end{array}$ & $\begin{array}{l}\text { USCE } \\
\text { USCE } \\
\text { USCE } \\
\text { USCE } \\
\text { USCE }\end{array}$ & $\begin{array}{l}72 \mathrm{~A} \\
\text { BF 170-75 } \\
\text { BF173-75 } \\
\text { GW99 } \\
\text { GW10OA }\end{array}$ \\
\hline $\begin{array}{l}\text { L025 } \\
\text { L026 } \\
\text { N028 } \\
\text { N029 } \\
0010\end{array}$ & $\begin{array}{l}\text { USCE } \\
\text { USCE } \\
\text { USCE } \\
\text { USCE } \\
\text { USCE }\end{array}$ & $\begin{array}{l}\text { GW101 } \\
\text { GW102 } \\
81 A \\
\text { GW95A } \\
\text { GW94 }\end{array}$ \\
\hline $\begin{array}{l}0011 \\
0012 \\
0013 \\
0014 \\
0015\end{array}$ & $\begin{array}{l}\text { USCE } \\
\text { USCE } \\
\text { USCE } \\
\text { USCE } \\
\text { USCE }\end{array}$ & $\begin{array}{l}\text { GW95 } \\
\text { GW96 } \\
\text { GW96A } \\
\text { GW97 } \\
\text { GW98 }\end{array}$ \\
\hline
\end{tabular}

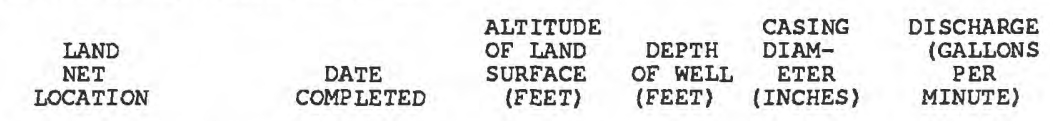

ALCORN COUNTY

$\begin{array}{lll}\text { NENES32T03SRO9E } & 04 / 28 / 1978 & 500 \\ \text { NESES32T03SR09E } & 05 / 12 / 1978 & 500 \\ \text { NWNES06T04SR09E } & 04 / 06 / 1978 & 600 \\ \text { NWNES06T04SR09E } & 04 / 13 / 1978 & 580 \\ \text { NENWS01T04SR08E } & 04 / 28 / 1980 & 590 \\ & & \\ \text { NENWS01T04SR08E } & 06 / 09 / 1980 & 590 \\ \text { SESES06T03SR09E } & 04 / 02 / 1981 & 600 \\ \text { SESES06T03SR09E } & 04 / 01 / 1981 & 600 \\ \text { SWSWS17T03SR09E } & 03 / 26 / 1982 & 600 \\ \text { SWSWS17T03SR09E } & --/--/ 1981 & 600 \\ & & \\ \text { SESES05T04SR09E } & 03 / 21 / 1982 & 537 \\ \text { SESES05T04SR09E } & 03 / 28 / 1983 & 537\end{array}$

$\begin{array}{llll}500 & 280 & 1.50 & -- \\ 500 & 207 & 1.50 & -- \\ 600 & 400 & 1.50 & -- \\ 580 & 327 & 1.50 & -- \\ 590 & 398 & 1.50 & - \\ 590 & 320 & 1.50 & - \\ 600 & 259 & 3 & - \\ 600 & 315 & 3 & - \\ 600 & 430 & 1.50 & -- \\ 600 & 380 & 1.50 & -- \\ 537 & 340 & 1.50 & -- \\ 537 & 263 & 3 & -\end{array}$

\begin{tabular}{|c|c|c|c|c|c|c|}
\hline $\begin{array}{l}211 \mathrm{MCSN} \\
110 \mathrm{ALVM} \\
110 \mathrm{ALVM} \\
110 \mathrm{ALVM} \\
\text { 211GORD }\end{array}$ & $\begin{array}{l}\text { SENES36T07SR08E } \\
\text { NESES35T07SR08E } \\
\text { SESES34T07SR08E } \\
\text { SWSWS29T07SRO9E } \\
\text { SWSWS21T07SR09E }\end{array}$ & $\begin{array}{l}07 / 08 / 1975 \\
07 / 10 / 1975 \\
07 / 14 / 1975 \\
07 / 16 / 1975 \\
05 / 16 / 1978\end{array}$ & $\begin{array}{l}325.70 \\
295 \\
297.20 \\
359.20 \\
440\end{array}$ & $\begin{array}{r}23 \\
38 \\
21 \\
21 \\
187\end{array}$ & $\begin{array}{l}1.50 \\
1.50 \\
1.50 \\
1.50 \\
1.50\end{array}$ & $\begin{array}{l}-- \\
-- \\
-- \\
--\end{array}$ \\
\hline $\begin{array}{l}\text { 211GORD } \\
\text { 211EUTW } \\
\text { 110ALVM } \\
\text { 110ALVM } \\
\text { 110ALVM }\end{array}$ & $\begin{array}{l}\text { NWNES28T07SR09E } \\
\text { NWSES 25T08SR08E } \\
\text { NENES12T09SR08E } \\
\text { NENWS25T08SR08E } \\
\text { SESES10T08SR08E }\end{array}$ & $\begin{array}{l}06 / 23 / 1978 \\
10 / 01 / 1975 \\
07 / 02 / 1975 \\
06 / 19 / 1975 \\
07 / 15 / 1975\end{array}$ & $\begin{array}{l}450 \\
271.10 \\
282.70 \\
275 \\
299.20\end{array}$ & $\begin{array}{r}200 \\
66 \\
23 \\
28 \\
29\end{array}$ & $\begin{array}{l}1.50 \\
1.50 \\
1.50 \\
1.50 \\
1.50\end{array}$ & $\begin{array}{l}=- \\
=- \\
=- \\
--\end{array}$ \\
\hline $\begin{array}{l}\text { 211EUTW } \\
110 A L V M \\
211 \mathrm{GORD} \\
211 \mathrm{GORD} \\
211 \mathrm{GORD}\end{array}$ & $\begin{array}{l}\text { SESWS06T08SR09E } \\
\text { SWSES01T08SR08E } \\
\text { SWSES25T08SR08E } \\
\text { SESES24T08SR08E } \\
\text { NESES18T08SR09E }\end{array}$ & $\begin{array}{l}07 / 08 / 1975 \\
06 / 17 / 1980 \\
08 / 28 / 1978 \\
05 / 01 / 1978 \\
05 / 18 / 1972\end{array}$ & $\begin{array}{l}333.50 \\
285 \\
290 \\
308.50 \\
325\end{array}$ & $\begin{array}{r}30 \\
25 \\
124 \\
152 \\
130\end{array}$ & $\begin{array}{l}1.50 \\
1.50 \\
1.50 \\
1.50 \\
4\end{array}$ & $\begin{array}{l}=- \\
=- \\
\overline{7.0}\end{array}$ \\
\hline $\begin{array}{l}\text { 110ALVM } \\
110 A L V M \\
211 \mathrm{GORD} \\
211 \mathrm{GORD} \\
\text { 211EUTW }\end{array}$ & $\begin{array}{l}\text { SWNES17T08SR09E } \\
\text { NESES18T08SR0 9E } \\
\text { NWNES30T08SRO 9E } \\
\text { SWSES27T09SR08E } \\
\text { SWSES27T09SR08E }\end{array}$ & $\begin{array}{l}07 / 07 / 1975 \\
07 / 03 / 1975 \\
05 / 15 / 1978 \\
08 / 12 / 1975 \\
08 / 13 / 1975\end{array}$ & $\begin{array}{l}329.50 \\
311.70 \\
420 \\
270 \\
270\end{array}$ & $\begin{array}{r}26 \\
12 \\
221 \\
179 \\
71\end{array}$ & $\begin{array}{l}1.50 \\
1.50 \\
1.50 \\
4 \\
4\end{array}$ & $\begin{array}{l}=- \\
=- \\
=-\end{array}$ \\
\hline $\begin{array}{l}211 \mathrm{GORD} \\
110 \mathrm{ALVM} \\
110 \mathrm{ALVM} \\
110 \mathrm{ALVM} \\
\text { 211GORD }\end{array}$ & $\begin{array}{l}\text { SWSES25T09SR08E } \\
\text { SWSES25T09SR08E } \\
\text { NWSWS26T09SR0BE } \\
\text { SWNWS25T09SR08E } \\
\text { NESWS36T0 9SR08E }\end{array}$ & $\begin{array}{l}04 / 19 / 1978 \\
04 / 19 / 1978 \\
01 / 01 / 1980 \\
04 / 12 / 1973 \\
04 / 20 / 1978\end{array}$ & $\begin{array}{l}284 \\
290 \\
260 \\
263.20 \\
290\end{array}$ & $\begin{array}{r}175 \\
10 \\
24 \\
33 \\
88\end{array}$ & $\begin{array}{l}6 \\
2 \\
2 \\
1.50 \\
1.50\end{array}$ & $\begin{array}{l}=- \\
=- \\
=-\end{array}$ \\
\hline $\begin{array}{l}\text { 211GORD } \\
110 \mathrm{ALVM} \\
110 \mathrm{ALVM} \\
\text { 211EUTW } \\
\text { 211GORD }\end{array}$ & $\begin{array}{l}\text { NESWS36T09SR08E } \\
\text { NESES34T09SR08E } \\
\text { SWSES27T09SR08E } \\
\text { NESWS13T09SR0BE } \\
\text { NESWS13T09SR08E }\end{array}$ & $\begin{array}{l}04 / 20 / 1978 \\
07 / 11 / 1975 \\
07 / 02 / 1975 \\
07 / 02 / 1975 \\
04 / 27 / 1978\end{array}$ & $\begin{array}{l}290 \\
254.60 \\
273.30 \\
284.50 \\
284.50\end{array}$ & $\begin{array}{r}138 \\
29 \\
31 \\
14 \\
150\end{array}$ & $\begin{array}{l}1.50 \\
1.50 \\
1.50 \\
1.50 \\
1.50\end{array}$ & $\begin{array}{l}=- \\
=- \\
=-\end{array}$ \\
\hline $\begin{array}{l}\text { 110ALVM } \\
211 \mathrm{GORD} \\
110 \mathrm{ALVM} \\
110 \mathrm{ALVM} \\
110 \mathrm{ALVM}\end{array}$ & $\begin{array}{l}\text { NWSWS15T09SR08E } \\
\text { NWSWS15T09SR08E } \\
\text { SESWS02T09SR08E } \\
\text { NWSWS03T09SR08E } \\
\text { SWNWS36T09SR08E }\end{array}$ & $\begin{array}{l}07 / 03 / 1975 \\
05 / 05 / 1978 \\
07 / 23 / 1975 \\
09 / 01 / 1975 \\
07 / 01 / 1975\end{array}$ & $\begin{array}{l}278.20 \\
278.20 \\
270.90 \\
292.90 \\
240\end{array}$ & $\begin{array}{r}24 \\
198 \\
35 \\
24 \\
26\end{array}$ & $\begin{array}{l}1.50 \\
1.50 \\
1.50 \\
1.50 \\
2\end{array}$ & $\begin{array}{l}=- \\
=- \\
=- \\
--\end{array}$ \\
\hline $\begin{array}{l}211 \mathrm{GORD} \\
110 \mathrm{ALVM} \\
211 \mathrm{GORD} \\
211 \mathrm{GORD} \\
211 \mathrm{GORD}\end{array}$ & $\begin{array}{l}\text { NENWS 24T1OSR08E } \\
\text { NENES24T1OSRO8E } \\
\text { NENES12T1OSRO8E } \\
\text { SWSWS17TIOSRO9E } \\
\text { SWSES17TIOSRO9E }\end{array}$ & $\begin{array}{l}06 / 20 / 1972 \\
06 / 24 / 1975 \\
01 / 14 / 1976 \\
06 / 22 / 1972 \\
06 / 22 / 1972\end{array}$ & $\begin{array}{l}273 \\
269.20 \\
250 \\
270 \\
300\end{array}$ & $\begin{array}{r}170 \\
21 \\
46 \\
150 \\
144\end{array}$ & $\begin{array}{l}4 \\
1.50 \\
2 \\
4 \\
4\end{array}$ & $\begin{array}{l}7.0 \\
200 \\
195\end{array}$ \\
\hline $\begin{array}{l}\text { 110ALVM } \\
211 \text { EUTW } \\
\text { 211GORD } \\
\text { 110ALVM } \\
\text { 110ALVM }\end{array}$ & $\begin{array}{l}\text { NWSES18TIOSRO9E } \\
\text { NESES19TIOSRO9E } \\
\text { NESES18T1OSRO9E } \\
\text { SWSES17TIOSRO9E } \\
\text { NWNWS18T1OSRO9E }\end{array}$ & $\begin{array}{l}06 / 11 / 1972 \\
08 / 20 / 1975 \\
07 / 15 / 1975 \\
06 / 26 / 1975 \\
07 / 08 / 1980\end{array}$ & $\begin{array}{l}249 \\
247.50 \\
252.20 \\
282.80 \\
255\end{array}$ & $\begin{array}{l}21 \\
67 \\
51 \\
30 \\
32\end{array}$ & $\begin{array}{l}4 \\
1.50 \\
1.50 \\
1.50 \\
1.50\end{array}$ & $\begin{array}{l}7.0 \\
=- \\
=- \\
--\end{array}$ \\
\hline $\begin{array}{l}\text { 11OALVM } \\
110 A L V M \\
211 \text { GORD } \\
110 A L V M \\
110 \text { ALVM }\end{array}$ & $\begin{array}{l}\text { SESWS06T1OSR09E } \\
\text { SESES01T1OSR08E } \\
\text { NESWS26TIISR08E } \\
\text { SENES23TIISRO8E } \\
\text { SESES20TIISRO9E }\end{array}$ & $\begin{array}{l}06 / 26 / 1975 \\
06 / 22 / 1975 \\
07 / 13 / 1972 \\
06 / 16 / 1975 \\
06 / 18 / 1975\end{array}$ & $\begin{array}{l}304.80 \\
277.10 \\
246 \\
242.40 \\
278\end{array}$ & $\begin{array}{r}21 \\
21 \\
180 \\
35 \\
23\end{array}$ & $\begin{array}{l}1.50 \\
1.50 \\
4 \\
1.50 \\
1.50\end{array}$ & $\begin{array}{l}-- \\
8.0 \\
--\end{array}$ \\
\hline $\begin{array}{l}\text { 11OALVM } \\
110 A L V M \\
11 O A L V M \\
11 O A L V M \\
11 O A L V M\end{array}$ & $\begin{array}{l}\text { SWSWS17TIISRO9E } \\
\text { NWSES18TIISRO9E } \\
\text { SWNWS20TIISRO9E } \\
\text { SESWS14TIOSRO9E } \\
\text { SWSWS31TIOSRO9E }\end{array}$ & $\begin{array}{l}06 / 19 / 1975 \\
06 / 20 / 1975 \\
06 / 22 / 1975 \\
06 / 25 / 1975 \\
06 / 23 / 1975\end{array}$ & $\begin{array}{l}325.40 \\
262.90 \\
238.60 \\
393.90 \\
267.20\end{array}$ & $\begin{array}{l}29 \\
23 \\
24 \\
60 \\
23\end{array}$ & $\begin{array}{l}1.50 \\
1.50 \\
1.50 \\
1.50 \\
1.50\end{array}$ & $\begin{array}{l}=- \\
\overline{--} \\
=-\end{array}$ \\
\hline
\end{tabular}


DESCRIPTIONS OF GROUND-WATER WELLS--Cont1nued

$\begin{array}{ll}\text { LOCAL } & \text { PRINCIPAL } \\ \text { NUMBER } & \text { OWNER }\end{array}$

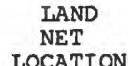

LOCATION
ALTITUDE
OF LAND
SURFACE (FEET) $\begin{array}{lcc} & \text { CASING } & \text { DISCHARGE } \\ \text { DEPTH } & \text { DIAM- } & \text { (GALLONS } \\ \text { OF WELL } & \text { ETER } & \text { PER } \\ \text { (EEET) } & \text { (INCHES) } & \text { MINUTE) }\end{array}$ MONROE COUNTY

\begin{tabular}{|c|c|c|c|c|c|c|c|c|c|}
\hline 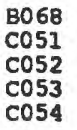 & $\begin{array}{l}\text { USCE } \\
\text { USCE } \\
\text { USCE } \\
\text { USCE } \\
\text { USCE }\end{array}$ & $\begin{array}{l}\text { APF58 } \\
84 \mathrm{~A} \\
84 \mathrm{~B} \\
84 \mathrm{C} \\
85 \mathrm{~A}\end{array}$ & $\begin{array}{l}211 \mathrm{EUTW} \\
211 \mathrm{GORD} \\
211 \mathrm{GORD} \\
11 \text { OALVM } \\
11 \text { OALVM }\end{array}$ & $\begin{array}{l}\text { NENWS34T1 2SR1 9W } \\
\text { NWNWS36T11 SROBE } \\
\text { NWNWS36T11 SRO8E } \\
\text { NWNWS36T11 } 1 \text { SR08E } \\
\text { SWNWS36T11 SROBE }\end{array}$ & $\begin{array}{l}01 / 15 / 1976 \\
06 / 29 / 1972 \\
07 / 10 / 1972 \\
07 / 10 / 1972 \\
07 / 11 / 1972\end{array}$ & $\begin{array}{l}210 \\
234 \\
234 \\
234 \\
235\end{array}$ & $\begin{array}{r}60 \\
170 \\
110 \\
27 \\
21\end{array}$ & $\begin{array}{l}2 \\
4 \\
4 \\
4 \\
4\end{array}$ & $\begin{array}{l}30- \\
10 \\
10 \\
18\end{array}$ \\
\hline $\begin{array}{l}\cos 7 \\
\cos 5 \\
\operatorname{co6} 1 \\
\operatorname{co6} 62 \\
\operatorname{co6} 68\end{array}$ & $\begin{array}{l}\text { USCE } \\
\text { USCE } \\
\text { USCE } \\
\text { USCE } \\
\text { USCE }\end{array}$ & $\begin{array}{l}89 A \\
89 B \\
91 A \\
92 A \\
92 B\end{array}$ & $\begin{array}{l}211 \mathrm{GORD} \\
211 \mathrm{MCSN} \\
211 \mathrm{MCSN} \\
211 \mathrm{GORD} \\
11 \text { OALVM }\end{array}$ & $\begin{array}{l}\text { SWNESO1T12SR08E } \\
\text { SWNES01T12SROBE } \\
\text { SWSWS17T12SR08E } \\
\text { NWSWS20T12SR08E } \\
\text { NWSWS20T1 2SRO8E }\end{array}$ & $\begin{array}{l}06 / 30 / 1972 \\
07 / 06 / 1972 \\
07 /--/ 1972 \\
07 /-/ 1972 \\
08 / 30 / 1972\end{array}$ & $\begin{array}{l}245 \\
245 \\
218 \\
216 \\
216\end{array}$ & $\begin{array}{r}166 \\
45 \\
88 \\
200 \\
19\end{array}$ & $\begin{array}{l}4 \\
4 \\
4 \\
4 \\
4\end{array}$ & $\begin{array}{c}20 \\
2.0 \\
-- \\
7.0\end{array}$ \\
\hline $\begin{array}{l}c 069 \\
C 070 \\
C 080 \\
C 081 \\
C 085\end{array}$ & $\begin{array}{l}\text { USCE } \\
\text { USCE } \\
\text { USCE } \\
\text { USCE } \\
\text { USCE }\end{array}$ & $\begin{array}{l}91 \mathrm{~B} \\
96 \mathrm{~B} \\
\text { TTM6A } \\
\text { TTM6B } \\
\text { A1A }\end{array}$ & $\begin{array}{l}\text { 11OALVM } \\
11 \text { TRCS } \\
211 \text { TBGB } \\
11 \text { OALVM } \\
\text { 11OALVM }\end{array}$ & $\begin{array}{l}\text { SWSWS17T1 2SROBE } \\
\text { SWSES20T1 2SR1 8W } \\
\text { NENES33T1 3SR19W } \\
\text { NENES33T13SR19W } \\
\text { SWSWS20T12SR08E }\end{array}$ & $\begin{array}{l}08 /-1 / 1972 \\
08 /-11972 \\
05 / 14 / 1975 \\
05 / 16 / 1975 \\
06 / 17 / 1982\end{array}$ & $\begin{array}{l}218 \\
257 \\
210 \\
210 \\
215\end{array}$ & $\begin{array}{r}20 \\
16 \\
65 \\
38 \\
5\end{array}$ & $\begin{array}{r}4 \\
24 \\
4 \\
6 \\
1.50\end{array}$ & $\begin{array}{l}4.0 \\
-- \\
--\end{array}$ \\
\hline $\begin{array}{l}\text { C086 } \\
\text { C087 } \\
\text { C088 } \\
\text { C089 } \\
\text { C090 }\end{array}$ & $\begin{array}{l}\text { USCE } \\
\text { USCE } \\
\text { USCE } \\
\text { USCE } \\
\text { USCE }\end{array}$ & $\begin{array}{l}\text { A1 } \\
\text { A2 } \\
\text { A2A } \\
\text { A3 } \\
\text { A3A }\end{array}$ & $\begin{array}{l}\text { 110ALVM } \\
110 A L V M \\
110 A L V M \\
110 A L V M \\
110 A L V M\end{array}$ & $\begin{array}{l}\text { SWSWS20T1 2SROBE } \\
\text { NWNWS29T12SROBE } \\
\text { NWNWS29T12SRO8E } \\
\text { NWNWS29T12SROBE } \\
\text { NWNWS29T12SROBE }\end{array}$ & $\begin{array}{l}06 / 17 / 1982 \\
06 / 18 / 1982 \\
06 / 18 / 1982 \\
06 / 21 / 1982 \\
07 / 01 / 1982\end{array}$ & $\begin{array}{l}215 \\
215 \\
215 \\
215 \\
215\end{array}$ & $\begin{array}{r}24 \\
29 \\
5 \\
25 \\
5\end{array}$ & $\begin{array}{l}1.50 \\
1.50 \\
1.50 \\
1.50 \\
1.00\end{array}$ & $=$ \\
\hline $\begin{array}{l}\operatorname{cog} 2 \\
\operatorname{cog} \\
\operatorname{cog} 4 \\
\operatorname{cog} \\
\operatorname{cog} 9\end{array}$ & $\begin{array}{l}\text { USCE } \\
\text { USCE } \\
\text { USCE } \\
\text { USCE } \\
\text { USCE }\end{array}$ & $\begin{array}{l}\text { GW80 } \\
\text { GW81 } \\
\text { GW83 } \\
\text { GW85 } \\
\text { GW86 }\end{array}$ & $\begin{array}{l}110 A L V M \\
110 A L V M \\
110 A L V M \\
110 A L V M \\
110 A L V M\end{array}$ & $\begin{array}{l}\text { SWNES35T12SR19W } \\
\text { NESES36T12SR19W } \\
\text { SESES30T12SROBE } \\
\text { SWSES20T1 2SR18W } \\
\text { NWNES19T12SR18W }\end{array}$ & $\begin{array}{l}05 / 28 / 1975 \\
05 / 29 / 1975 \\
06 / 02 / 1975 \\
06 / 10 / 1975 \\
06 / 09 / 1975\end{array}$ & $\begin{array}{l}236.40 \\
235.70 \\
213.20 \\
257.60 \\
247.10\end{array}$ & $\begin{array}{l}33 \\
25 \\
33 \\
26 \\
24\end{array}$ & $\begin{array}{l}1.50 \\
1.50 \\
1.50 \\
1.50 \\
1.50\end{array}$ & $=$ \\
\hline $\begin{array}{l}\text { C097 } \\
\text { C098 } \\
\text { c099 } \\
\text { C100 } \\
\text { C101 }\end{array}$ & $\begin{array}{l}\text { USCE } \\
\text { USCE } \\
\text { USCE } \\
\text { USCE } \\
\text { USCE }\end{array}$ & $\begin{array}{l}\text { GW86A } \\
\text { GW87 } \\
\text { GW88 } \\
\text { GW89 } \\
\text { GW90 }\end{array}$ & $\begin{array}{l}110 \text { ALVM } \\
110 A L V M \\
110 A L V M \\
110 A L V M \\
110 A L V M\end{array}$ & $\begin{array}{l}\text { NESWS21T12SR08E } \\
\text { SENWS16T12SR1 BW } \\
\text { SWSWS15T12SR08E } \\
\text { SESWS09T12SR08E } \\
\text { NENWS13T12SR08E }\end{array}$ & $\begin{array}{l}06 / 23 / 1980 \\
06 / 18 / 1975 \\
06 / 05 / 1975 \\
06 / 04 / 1975 \\
06 / 13 / 1975\end{array}$ & $\begin{array}{l}210 \\
258 \\
244.20 \\
225 \cdot 20 \\
270\end{array}$ & $\begin{array}{l}31 \\
30 \\
24 \\
30 \\
26\end{array}$ & $\begin{array}{l}1.50 \\
1.50 \\
1.50 \\
1.50 \\
1.50\end{array}$ & $\begin{array}{l}-- \\
-- \\
--\end{array}$ \\
\hline $\begin{array}{l}\mathrm{C} 102 \\
\mathrm{C} 105 \\
\mathrm{C} 106 \\
\mathrm{C} 107 \\
\mathrm{C} 108\end{array}$ & $\begin{array}{l}\text { USCE } \\
\text { USCE } \\
\text { USCE } \\
\text { USCE } \\
\text { USCE }\end{array}$ & $\begin{array}{l}\text { GW91 } \\
\text { GW92B } \\
\text { GW92C } \\
\text { LA2L } \\
\text { LA2U }\end{array}$ & $\begin{array}{l}\text { 110ALVM } \\
211 \text { EUTW } \\
211 \text { EUTW } \\
211 \text { GORD } \\
211 \text { EUTW }\end{array}$ & $\begin{array}{l}\text { SENES11T1 2SR08E } \\
\text { NWSWSO1T12SR08E } \\
\text { NWSWS01T12SR08E } \\
\text { SWNWS24T12SR1 9W } \\
\text { SWNWS24T12SR19W }\end{array}$ & $\begin{array}{l}06 / 12 / 1975 \\
04 / 14 / 1975 \\
04 / 14 / 1975 \\
05 / 01 / 1975 \\
05 / 02 / 1975\end{array}$ & $\begin{array}{l}261.30 \\
257.30 \\
257.40 \\
220 \\
220\end{array}$ & $\begin{array}{r}23 \\
122 \\
100 \\
147 \\
67\end{array}$ & $\begin{array}{l}1.50 \\
1.50 \\
1.50 \\
1.50 \\
1.50\end{array}$ & $\begin{array}{l}-- \\
-- \\
--\end{array}$ \\
\hline $\begin{array}{l}\text { C109 } \\
\text { C110 } \\
\text { C111 } \\
\text { C112 } \\
\text { C113 }\end{array}$ & $\begin{array}{l}\text { USCE } \\
\text { USCE } \\
\text { USCE } \\
\text { USCE } \\
\text { USCE }\end{array}$ & $\begin{array}{l}\text { IA } 4 L \\
\text { IA4U } \\
\text { IASI } \\
\text { LA5U } \\
\text { AF } 63\end{array}$ & $\begin{array}{l}211 \mathrm{GORD} \\
211 \mathrm{EUTW} \\
211 \mathrm{GORD} \\
211 \mathrm{EUTW} \\
211 \mathrm{EUTW}\end{array}$ & $\begin{array}{l}\text { NESES36T1 2SR19W } \\
\text { NESES36T1 2SR19W } \\
\text { SWSES30T1.2SR08E } \\
\text { SWSES30T12SR08E } \\
\text { SWSESO3T1 2SR08E }\end{array}$ & $\begin{array}{l}05 / 03 / 1975 \\
05 / 04 / 1975 \\
05 / 05 / 1975 \\
05 / 06 / 1975 \\
01 / 20 / 1976\end{array}$ & $\begin{array}{l}235 \\
235 \\
210 \\
210 \\
230\end{array}$ & $\begin{array}{r}190 \\
122 \\
205 \\
57 \\
54\end{array}$ & $\begin{array}{l}1.50 \\
1.50 \\
1.50 \\
1.50 \\
2\end{array}$ & $\begin{array}{l}-- \\
-- \\
--\end{array}$ \\
\hline $\begin{array}{l}\text { D032 } \\
\text { D033 } \\
\text { D034 } \\
\text { D035 } \\
\text { D036 }\end{array}$ & $\begin{array}{l}\text { USCE } \\
\text { USCE } \\
\text { USCE } \\
\text { USCE } \\
\text { USCE }\end{array}$ & $\begin{array}{l}\text { GW92 } \\
\text { GW93A } \\
\text { GW93B } \\
\text { GW93C } \\
\text { GW94A }\end{array}$ & $\begin{array}{l}110 \text { ALVM } \\
211 \text { GORD } \\
211 \text { EUTW } \\
11 \text { ALVM } \\
110 \text { ALVM }\end{array}$ & $\begin{array}{l}\text { NESWSO6T1.2SR09E } \\
\text { SWNWSO6T12SR09E } \\
\text { SWNWSO6T12SR09E } \\
\text { SWNWSO6T1 2SR09E } \\
\text { NWNWSO6T12SR09E }\end{array}$ & $\begin{array}{l}06 / 11 / 1975 \\
05 / 15 / 1975 \\
05 / 15 / 1975 \\
05 / 15 / 1975 \\
06 / 26 / 1975\end{array}$ & $\begin{array}{l}266.70 \\
259.20 \\
259.40 \\
259.40 \\
255\end{array}$ & $\begin{array}{r}20 \\
178 \\
102 \\
20 \\
27\end{array}$ & $\begin{array}{l}1.50 \\
1.50 \\
1.50 \\
1.50 \\
1.50\end{array}$ & $\begin{array}{l}-- \\
-- \\
--\end{array}$ \\
\hline $\begin{array}{l}\text { G052 } \\
\text { G053 } \\
\text { G054 } \\
\text { G055 } \\
\text { G056 }\end{array}$ & $\begin{array}{l}\text { USCE } \\
\text { USCE } \\
\text { USCE } \\
\text { USCE } \\
\text { USCE }\end{array}$ & $\begin{array}{l}\text { APF55 } \\
\text { APF57 } \\
\text { GW73 } \\
\text { GW74 } \\
\text { GW76 }\end{array}$ & $\begin{array}{l}\text { 211EUTW } \\
211 E U T W \\
110 A L V M \\
110 A L V M \\
110 A L V M\end{array}$ & $\begin{array}{l}\text { NESWS03T13SR1 9W } \\
\text { SESWS34T12SR19W } \\
\text { SESWS27T13SR07E } \\
\text { SWNWS36T13SR19W } \\
\text { NWNES10T13SR19W }\end{array}$ & $\begin{array}{l}08 / 05 / 1975 \\
07 / 22 / 1975 \\
05 / 23 / 1975 \\
05 / 26 / 1975 \\
06 / 02 / 1975\end{array}$ & $\begin{array}{l}205.70 \\
207.30 \\
198 \\
214.30 \\
203.40\end{array}$ & $\begin{array}{l}69 \\
50 \\
32 \\
32 \\
45\end{array}$ & $\begin{array}{l}1.50 \\
1.50 \\
1.50 \\
1.50 \\
1.50\end{array}$ & $\begin{array}{l}-- \\
=- \\
--\end{array}$ \\
\hline $\begin{array}{l}\text { G057 } \\
\text { G058 } \\
\text { G059 } \\
\text { G060 } \\
\text { H018 }\end{array}$ & $\begin{array}{l}\text { USCE } \\
\text { USCE } \\
\text { USCE } \\
\text { USCE } \\
\text { USCE }\end{array}$ & $\begin{array}{l}\text { GW76A } \\
\text { GW77 } \\
\text { GW78 } \\
\text { GW75 } \\
\text { TTM5B }\end{array}$ & $\begin{array}{l}110 \text { ALVM } \\
110 \text { ALVM } \\
110 A L V M \\
110 A L V M \\
110 A L V M\end{array}$ & $\begin{array}{l}\text { NWSES15T13SR07E } \\
\text { NENES11T13SR19W } \\
\text { NENWSO3T13SRO 7E } \\
\text { NWSES 23T13SR19W } \\
\text { SESWS22T13SR19W }\end{array}$ & $\begin{array}{l}06 / 18 / 1975 \\
05 / 31 / 1975 \\
05 / 20 / 1975 \\
05 / 21 / 1975 \\
05 / 08 / 1975\end{array}$ & $\begin{array}{l}202.40 \\
234.20 \\
205.30 \\
200 \\
200\end{array}$ & $\begin{array}{l}25 \\
46 \\
36 \\
30 \\
26\end{array}$ & $\begin{array}{l}1.50 \\
1.50 \\
1.50 \\
2 \\
6\end{array}$ & $=$ \\
\hline $\begin{array}{l}1062 \\
L 063 \\
\text { L064 } \\
\text { L065 } \\
\text { L067 }\end{array}$ & $\begin{array}{l}\text { USCE } \\
\text { USCE } \\
\text { USCE } \\
\text { USCE } \\
\text { USCE }\end{array}$ & $\begin{array}{l}105 \mathrm{~A} \\
101 \mathrm{~A} \\
102 \mathrm{~A} \\
104 \mathrm{~A} \\
104 \mathrm{~B}\end{array}$ & $\begin{array}{l}\text { 211EUTW } \\
211 \text { EUTW } \\
211 E U T W \\
211 \text { EUTW } \\
110 A L V M\end{array}$ & $\begin{array}{l}\text { SWSWS19T1 4SR1 9W } \\
\text { SWSWS15T1 4SR07E } \\
\text { NWNWS23T1 4SRO7E } \\
\text { SESES10T1 4SR1 9W } \\
\text { SESES10T1 4SR19E. }\end{array}$ & $\begin{array}{l}08 / 11 / 1972 \\
08 / 09 / 1972 \\
08 / 04 / 1972 \\
08 / 14 / 1972 \\
08 / 15 / 1972\end{array}$ & $\begin{array}{l}210 \\
202 \\
191 \\
194 \\
194\end{array}$ & $\begin{array}{l}64 \\
90 \\
50 \\
55 \\
24\end{array}$ & $\begin{array}{l}4 \\
4 \\
4 \\
4 \\
4\end{array}$ & $\begin{array}{l}-- \\
-- \\
-\end{array}$ \\
\hline $\begin{array}{l}L 068 \\
L 069 \\
L 075 \\
L 077 \\
L 078\end{array}$ & $\begin{array}{l}\text { USCE } \\
\text { USCE } \\
\text { USCE } \\
\text { USCE } \\
\text { USCE }\end{array}$ & $\begin{array}{l}102 \mathrm{~B} \\
101 \mathrm{~B} \\
\mathrm{AB} 11 \\
\mathrm{AB} 10 \\
\mathrm{AB} 12\end{array}$ & $\begin{array}{l}\text { 110ALVM } \\
110 T R C S \\
211 \text { MCSN } \\
211 \text { EUTW } \\
\text { 211EUTW }\end{array}$ & $\begin{array}{l}\text { NWWWS23T1 4SRO7E } \\
\text { SWSWS15T1 4SRO7E } \\
\text { SWSWS26T1 4SRO7E } \\
\text { SWSWS26T1 4SRO7E } \\
\text { SWSWS26T1 4SR07E }\end{array}$ & $\begin{array}{l}08 / 07 / 1972 \\
08 / 09 / 1972 \\
12 / 11 / 1976 \\
12 / 15 / 1976 \\
12 / 17 / 1976\end{array}$ & $\begin{array}{l}191 \\
202 \\
200 \\
200 \\
200\end{array}$ & $\begin{array}{r}30 \\
20 \\
224 \\
145 \\
90\end{array}$ & $\begin{array}{l}4 \\
4 \\
4 \\
4 \\
4\end{array}$ & $\begin{array}{l}-- \\
-- \\
-- \\
--\end{array}$ \\
\hline $\begin{array}{l}\text { L084 } \\
\text { L085 } \\
\text { L086 } \\
\text { L087 } \\
\text { L088 }\end{array}$ & $\begin{array}{l}\text { USCE } \\
\text { USCE } \\
\text { USCE } \\
\text { USCE } \\
\text { USCE }\end{array}$ & $\begin{array}{l}\text { AB1 } \\
\text { AB2 } \\
\text { AB2A } \\
\text { AB5 } \\
\text { AB6 }\end{array}$ & $\begin{array}{l}\text { 211EUTW } \\
\text { 211EUTW } \\
\text { 211EUTW } \\
\text { 211EUTW } \\
\text { 211EUTW }\end{array}$ & $\begin{array}{l}\text { NWSWS 26T1 4SRO7E } \\
\text { NWSWS26T1 4SRO7E } \\
\text { NWSWS26T1 4SRO } \\
\text { NESES10T1 4SR1 9W } \\
\text { NWSWS26T1 4SRO7E }\end{array}$ & $\begin{array}{l}10 / 28 / 1976 \\
10 / 28 / 1976 \\
11 / 06 / 1976 \\
10 / 14 / 1976 \\
11 / 07 / 1976\end{array}$ & $\begin{array}{l}195 \\
195 \\
195 \\
192 \\
190\end{array}$ & $\begin{array}{r}80 \\
146 \\
119 \\
150 \\
70\end{array}$ & $\begin{array}{l}1.50 \\
1.50 \\
1.50 \\
1.50 \\
1.50\end{array}$ & $\begin{array}{l}-- \\
-- \\
-- \\
--\end{array}$ \\
\hline
\end{tabular}




\begin{tabular}{|c|c|c|c|}
\hline $\begin{array}{l}\text { LOCAL } \\
\text { NUMBER }\end{array}$ & & OWNER & $\begin{array}{l}\text { PRINCIPAL } \\
\text { AQUIFER }\end{array}$ \\
\hline $\begin{array}{l}1089 \\
1090 \\
1091 \\
2092 \\
L 093\end{array}$ & $\begin{array}{l}\text { USCE } \\
\text { USCE } \\
\text { USCE } \\
\text { USCE } \\
\text { USCE }\end{array}$ & $\begin{array}{l}\text { AB7 } \\
\text { GW70 } \\
\text { GW70A } \\
\text { GW71 } \\
\text { GW72 }\end{array}$ & $\begin{array}{l}\text { 211EUTW } \\
110 \mathrm{ALVM} \\
110 \mathrm{ALVM} \\
110 \mathrm{ALVM} \\
110 \mathrm{ALVM}\end{array}$ \\
\hline $\begin{array}{l}\text { L095 } \\
\text { L096 }\end{array}$ & $\begin{array}{l}\text { USCE } \\
\text { USCE }\end{array}$ & $\begin{array}{l}\text { P1 } \\
\text { P2 }\end{array}$ & $\begin{array}{l}\text { 211EUTW } \\
\text { 211EUTW }\end{array}$ \\
\hline $\begin{array}{l}\text { DO28 } \\
\text { DO30 } \\
\text { D032 } \\
\text { D033 } \\
\text { D036 }\end{array}$ & $\begin{array}{l}\text { USCE } \\
\text { USCE } \\
\text { USCE } \\
\text { USCE } \\
\text { USCE }\end{array}$ & $\begin{array}{l}M W 1-2 \\
M W 1-4 \\
M W 2-2 \\
M W 2-3 \\
W 1-1\end{array}$ & $\begin{array}{l}\text { 211EUTW } \\
211 G O R D \\
211 G O R D \\
211 E U T W \\
211 G O R D\end{array}$ \\
\hline $\begin{array}{l}\text { D037 } \\
\text { DO39 } \\
\text { DO 40 } \\
\text { HO26 } \\
\text { H028 }\end{array}$ & $\begin{array}{l}\text { USCE } \\
\text { USCE } \\
\text { USCE } \\
\text { USCE } \\
\text { USCE }\end{array}$ & $\begin{array}{l}\text { W1-2 } \\
\text { W3-1 } \\
\text { W3-2 } \\
\text { SW2-3 } \\
\text { SW3-2 }\end{array}$ & $\begin{array}{l}\text { 211EUTW } \\
211 \mathrm{GORD} \\
211 \mathrm{EUTW} \\
211 \mathrm{GORD} \\
211 \mathrm{GORD}\end{array}$ \\
\hline $\begin{array}{l}\text { HO29 } \\
\text { HO31 } \\
\text { M033 } \\
\text { MO16 } \\
\text { M017 }\end{array}$ & $\begin{array}{l}\text { USCE } \\
\text { USCE } \\
\text { USCE } \\
\text { USCE } \\
\text { USCE }\end{array}$ & $\begin{array}{l}\text { SW3-3 } \\
\text { SW2-4 } \\
\text { W6-2 } \\
53 A \\
43 C\end{array}$ & $\begin{array}{l}211 \mathrm{EUTW} \\
211 \mathrm{MCSN} \\
211 \mathrm{GORD} \\
211 \mathrm{GORD} \\
211 \mathrm{EUTWR}\end{array}$ \\
\hline $\begin{array}{l}\text { MO18 } \\
\text { MO19 } \\
\text { MO20 } \\
\text { MO21 } \\
\text { MO22 }\end{array}$ & $\begin{array}{l}\text { USCE } \\
\text { USCE } \\
\text { USCE } \\
\text { USCE } \\
\text { USCE }\end{array}$ & $\begin{array}{l}43 B \\
52 A \\
51 A \\
41 A \\
41 B\end{array}$ & $\begin{array}{l}\text { 211MCSN } \\
211 \text { GORD } \\
211 \text { GORD } \\
211 \text { GORD } \\
\text { 211EUTW }\end{array}$ \\
\hline $\begin{array}{l}\text { MO23 } \\
\text { MO25 } \\
\text { MO26 } \\
\text { MO27 } \\
\text { MO28 }\end{array}$ & $\begin{array}{l}\text { USCE } \\
\text { USCE } \\
\text { USCE } \\
\text { USCE } \\
\text { USCE }\end{array}$ & $\begin{array}{l}43 A \\
43 D \\
42 A \\
42 B \\
\text { GW123A }\end{array}$ & $\begin{array}{l}\text { 211GORD } \\
\text { 211EUTWR } \\
\text { 211EUTW } \\
\text { 211EUTW } \\
\text { 110TRCS }\end{array}$ \\
\hline $\begin{array}{l}\text { M030 } \\
\text { MO31 } \\
\text { MO32 }\end{array}$ & $\begin{array}{l}\text { USCE } \\
\text { USCE } \\
\text { USCE }\end{array}$ & $\begin{array}{l}\text { GW122 } \\
\text { GW123 } \\
\text { GW124 }\end{array}$ & $\begin{array}{l}\text { 110ALVM } \\
110 \mathrm{ALVM} \\
110 \mathrm{ALVM}\end{array}$ \\
\hline
\end{tabular}

\section{A017}

A019

$\mathrm{A} 020$

D037
D0 40

D041

D042

D0 44

$D 047$
$D 048$

D050

D051

D055

D056
D059

D0 60

D064

D065

D068

D069

E014

E015

E027

E028

E033

E034

E041
USCE 10DP177

USCE 2MW16

USCE 2MW17

USCE 14A

USCE 12B

USCE $12 \mathrm{C}$

USCE $14 \mathrm{C}$

USCE 1DP141

USCE 1DP142

USCE $11 \mathrm{C}$

USCE 110

USCE 3DP151

USCE 3DP152

USCE 7DP167

USCE 7DP1 68

USCE W2-3

USCE W2-4

USCE 2MW6

USCE 2MW7

USCE 2MW8

USCE 15A

USCE 15B

USCE NE2-3

USCE NE2-4

USCE NE3-4

USCE NE3-5

USCE E2-1
211GORD

211EUTWR

211EUTWR

211EUTW

211EUTWR

211EUTWR

211GORD

211EUTW

211GORD

211EUTW

211GORD

211EUTW

211GORD

211EUTW

(11GORD

211GORD

211GORD

211GORD

211EUTWR

211GORD

211GORD

211EUTW

211GORD

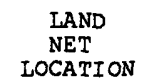

ALTITUDE

LOCATION

DATE

COMPLETED

SUREACE

(FEET)

MONROE COUNTY--COnt1nued

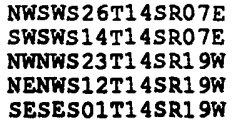

SESESO1T14SR1 9W

NENWS35T1 4SR1 9W
NENWS35T14SR1 9W

$\begin{array}{ll}11 / 07 / 1976 & 187.40 \\ 05 / 28 / 1975 & 193.30 \\ 06 / 17 / 1980 & 193 \\ 05 / 15 / 1975 & 225.40 \\ 05 / 19 / 1975 & 220.80 \\ & \\ 10 / 05 / 1982 & 190 \\ 10 / 01 / 1982 & 190\end{array}$

PRENTISS COUNTY

\begin{tabular}{|c|c|c|}
\hline $\begin{array}{l}\text { NWNES33T04SRO 9E } \\
\text { NENES33TO4SRO9E } \\
\text { NESES31TO4SRO9E } \\
\text { NESES3ITO } 4 S R O 9 E \\
\text { SWNWSO8TO4SRO9E }\end{array}$ & $\begin{array}{l}08 / 18 / 1977 \\
09 / 04 / 1978 \\
03 / 18 / 1980 \\
03 / 25 / 1980 \\
07 / 07 / 1980\end{array}$ & $\begin{array}{l}510 \\
510 \\
440 \\
440 \\
580\end{array}$ \\
\hline $\begin{array}{l}\text { SWNWS08T0 4SRO9E } \\
\text { SESWS15TO4SRO9E } \\
\text { SESWS15T04SRO9E } \\
\text { SWSES10TO5SRO9E } \\
\text { SWNWS16TO5SRO9E }\end{array}$ & $\begin{array}{l}07 / 07 / 1980 \\
12 / 04 / 1980 \\
12 / 10 / 1980 \\
12 / 13 / 1977 \\
02 / 04 / 1980\end{array}$ & $\begin{array}{l}580 \\
500 \\
500 \\
480 \\
450\end{array}$ \\
\hline $\begin{array}{l}\text { SWNWS16T05SRO9E } \\
\text { SWSES10T05SR09E } \\
\text { SESES10T05SRO9E } \\
\text { NWSES10T07SRO9E } \\
\text { NWNWS27T06SRO9E }\end{array}$ & $\begin{array}{l}07 / 02 / 1980 \\
01 / 01 / 1977 \\
03 / 04 / 1982 \\
05 /--/ 1972 \\
05 /--/ 1972\end{array}$ & $\begin{array}{l}450 \\
480 \\
480 \\
332 \\
445\end{array}$ \\
\hline $\begin{array}{l}\text { NWNWS 27T06SR09E } \\
\text { NESESO9T07SRO9E } \\
\text { SENWS09T07SR09E } \\
\text { SENWS28T06SRO9E } \\
\text { SENWS28TO } 6 S R 09 E\end{array}$ & $\begin{array}{l}05 /--/ 1972 \\
05 /=-/ 1972 \\
05 /=-/ 1972 \\
05 /=-/ 1972 \\
05 /=-/ 1972\end{array}$ & $\begin{array}{l}460 \\
324 \\
356 \\
480 \\
480\end{array}$ \\
\hline $\begin{array}{l}\text { NWNWS27T0 6SR09E } \\
\text { NWNWS27TO6SRO9E } \\
\text { NENES28TO6SRO9E } \\
\text { NENES28TO6SRO9E } \\
\text { NWNWS16T07SRO9E }\end{array}$ & $\begin{array}{l}05 /--/ 1972 \\
05 /--/ 1975 \\
05 /=-/ 1975 \\
05 /--/ 1975 \\
01 / 01 / 1980\end{array}$ & $\begin{array}{l}460 \\
460 \\
420 \\
420 \\
316\end{array}$ \\
\hline $\begin{array}{l}\text { SWSES07T07SR09E } \\
\text { SESWS15TO7SR09E } \\
\text { SENWS09T07SRO9E }\end{array}$ & $\begin{array}{l}02 / 21 / 1975 \\
07 / 15 / 1975 \\
07 / 18 / 1975\end{array}$ & $\begin{array}{l}330 \\
354 \\
350\end{array}$ \\
\hline
\end{tabular}

158
285
220
181
371
280
215
180
217
192
132
133
162
35
90
120
40
64
226
176
170
118
69
49
23
23
22
39

TISHMINGO COUNTY

211GORD NENAS36TO2SRO

211EUTW NWSES35T02SRO9E

211GORD NWSES35TO2SRO9E

211GORD SENWS36T03SRO9

$11 / 03 / 1980$

$11 / 21 / 1979$
$05 / 14 / 1973$

$--/--/ 1981$

$03 / 06 / 1981$

$03 / 09 / 1972$

$03 / 17 / 1972$

$03 / 21 / 1972$

$02 / 29 / 1972$

$05 /--/ 1972$

$05 /--/ 1972$

$10 / 21 / 1975$

$10 / 21 / 1976$

$08 / 30 / 1972$

$09 / 11 / 1972$

$03 / 12 / 1973$

$03 / 13 / 1973$

$11 / 06 / 1980$

$--/--/ 1981$

$--/--/ 1981$

$02 / 15 / 1972$

$02 / 24 / 1972$

$02 /--/ 1972$

$03 / 07 / 1970$

$03 / 24 / 1978$

$11 / 27 / 1979$

$03 / 08 / 1983$
433.40

500
500

500
545

485

SESWS 26TO3SR10E

SESWS 26T03SR10E

SESES2OTO3SR1OE
485

485

545
464.50

462.5

505

505

453.30
451.40

451.

446.60

480
479

475

455

475

540
540

540
540

580

580

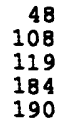

150

88

106
134

57

404

210

163

88

43

172
104

90

110

131

340
204

130

157

340

585
585

550.40
550.20

$\begin{array}{ccc} & \text { CASING } & \text { DISCHARGE } \\ \text { DEPTH } & \text { DIAM- } & \text { (GALLONS } \\ \text { OF WELL } & \text { ETER } & \text { PER } \\ \text { (EEET) } & \text { (INCHES) } & \text { MINUTE) }\end{array}$

$\begin{array}{rll}133 & 1.50 & = \\ 26 & 1.50 & = \\ 27 & 1.50 & = \\ 32 & 1.50 & = \\ 30 & 1.50 & = \\ 38 & 1.50 & -- \\ 42 & 1.50 & =\end{array}$

$\begin{array}{ll}1.50 & -- \\ 1.50 & =- \\ 1.50 & =- \\ 1.50 & =- \\ 1.50 & -- \\ 1.50 & -- \\ 1.50 & =- \\ 1.50 & -- \\ 1.50 & =- \\ 1.50 & -- \\ 1.50 & -- \\ 1.50 & -- \\ 1.50 & -- \\ 4 & 3.0 \\ 4 & -- \\ 4 & -- \\ 4 & 20 \\ 4 & 6.0 \\ 4 & -- \\ 4 & 16 \\ 4 & -- \\ 4 & -- \\ 4 & -- \\ 4 & -- \\ 2 & -- \\ 2 & -- \\ 2 & -- \\ 2 & --\end{array}$

$8 \quad 60$

2.0 


\begin{tabular}{|c|c|c|c|c|c|c|c|c|c|}
\hline $\begin{array}{l}\text { LOCAL } \\
\text { NUMBER }\end{array}$ & & OWNER & $\begin{array}{l}\text { PRINCIPAL } \\
\text { AQUIFER }\end{array}$ & $\begin{array}{l}\text { LAND } \\
\text { NET } \\
\text { LOCATION }\end{array}$ & $\begin{array}{c}\text { DATE } \\
\text { COMP LETED }\end{array}$ & $\begin{array}{l}\text { ALTITUDE } \\
\text { OF IAND } \\
\text { SURFACE } \\
\text { (FEET) }\end{array}$ & $\begin{array}{l}\text { DEPTH } \\
\text { OF WELL } \\
\text { (FEET) }\end{array}$ & $\begin{array}{l}\text { CASING } \\
\text { DIAM- } \\
\text { ETER } \\
\text { (INCHES) }\end{array}$ & $\begin{array}{c}\text { DISCHARG) } \\
\text { (GALLON } \\
\text { PER } \\
\text { MINUTE) }\end{array}$ \\
\hline \multicolumn{10}{|c|}{ TISHOMINGO COUNTY--Cont Inued } \\
\hline $\begin{array}{l}E 042 \\
\text { E043 } \\
\text { E044 } \\
\text { E045 } \\
\text { GOO4 }\end{array}$ & $\begin{array}{l}\text { USCE } \\
\text { USCE } \\
\text { USCE } \\
\text { USCE } \\
\text { USCE }\end{array}$ & $\begin{array}{l}2 M W 9 \\
2 M W 10 \\
2 M W 13 \\
2 M W 14 \\
21 A\end{array}$ & $\begin{array}{l}\text { 211EUTW } \\
\text { 211EUTW } \\
\text { 211EUTH } \\
211 \text { GORD } \\
\text { 211GORD }\end{array}$ & $\begin{array}{l}\text { SENWSO6TO3SR1 OE } \\
\text { SENWSO 6T03SRI OE } \\
\text { NWSWS 36T02SRO 9E } \\
\text { NWSWS36TO2SRO 9E } \\
\text { SWSWS26T04SRO9E }\end{array}$ & $\begin{array}{l}--/--/ 1981 \\
--/--/ 1973 \\
--/--/ 1981 \\
--/--/ 1981 \\
05 / 24 / 1971\end{array}$ & $\begin{array}{l}450 \\
450 \\
472 \\
472 \\
585\end{array}$ & $\begin{array}{r}36 \\
42 \\
88 \\
99 \\
278\end{array}$ & $\begin{array}{l}1.50 \\
3 \\
1.50 \\
1.50 \\
4\end{array}$ & $\begin{array}{l}=- \\
=- \\
=- \\
3.0\end{array}$ \\
\hline $\begin{array}{l}\text { G005 } \\
\text { G013 } \\
\text { G014 } \\
\text { G015 } \\
\text { G016 }\end{array}$ & $\begin{array}{l}\text { USCE } \\
\text { USCE } \\
\text { USCE } \\
\text { USCE } \\
\text { USCE }\end{array}$ & $\begin{array}{l}21 \mathrm{~B} \\
35 \mathrm{~A} \\
35 \mathrm{~B} \\
25 \mathrm{~A} \\
25 \mathrm{~B}\end{array}$ & $\begin{array}{l}\text { 211EUTWL } \\
211 \text { GORD } \\
211 \text { EUTWR } \\
211 \text { GORD } \\
\text { 211EUTWR }\end{array}$ & $\begin{array}{l}\text { SWSWS26T04SRO 9E } \\
\text { NESWS33T04SRI OE } \\
\text { NESWS33TO4SRI OE } \\
\text { NWSWS2OTO4SRI OE } \\
\text { NWSWS2OTO4SR1OE }\end{array}$ & $\begin{array}{l}05 /--/ 1971 \\
07 /--11971 \\
07 / 22 / 1971 \\
07 / 28 / 1971 \\
08 / 03 / 1971\end{array}$ & $\begin{array}{l}585 \\
600 \\
600 \\
610 \\
610\end{array}$ & $\begin{array}{l}235 \\
300 \\
203 \\
235 \\
200\end{array}$ & $\begin{array}{l}4 \\
4 \\
4 \\
4 \\
4\end{array}$ & $\begin{array}{l}11 \\
6.0 \\
5.0 \\
30\end{array}$ \\
\hline $\begin{array}{l}\text { G017 } \\
\text { G018 } \\
\text { G019 } \\
\text { G020 } \\
\text { G023 }\end{array}$ & $\begin{array}{l}\text { USCE } \\
\text { USCE } \\
\text { USCE } \\
\text { USCE } \\
\text { USCE }\end{array}$ & $\begin{array}{l}26 \mathrm{~A} \\
26 \mathrm{~B} \\
26 \mathrm{C} \\
23 \mathrm{C} \\
23 \mathrm{G}\end{array}$ & $\begin{array}{l}211 \text { GORD } \\
211 \text { EUTWR } \\
211 \text { EUTWR } \\
211 \text { GORD } \\
211 \text { GORD }\end{array}$ & $\begin{array}{l}\text { NESES2OTO4SR1OE } \\
\text { NESES2OTO 4SR1OE } \\
\text { NESES2OTO 4SR1OE } \\
\text { NWNWS 3OTO 4SR1OE } \\
\text { NWNWS 3OTO4SR1OE }\end{array}$ & $\begin{array}{l}07 / 28 / 1971 \\
07 /--/ 1971 \\
07 /--/ 1971 \\
08 / 25 / 1971 \\
09 / 15 / 1971\end{array}$ & $\begin{array}{l}565 \\
565 \\
565 \\
588 \\
601.3\end{array}$ & $\begin{array}{r}250 \\
127 \\
72 \\
330 \\
260\end{array}$ & $\begin{array}{l}2 \\
4 \\
2 \\
2 \\
2\end{array}$ & $\begin{array}{l}=- \\
\overline{5.0} \\
=- \\
--\end{array}$ \\
\hline $\begin{array}{l}\text { GO27 } \\
\text { GO31 } \\
\text { GO32 } \\
\text { GO33 } \\
\text { G034 }\end{array}$ & $\begin{array}{l}\text { USCE } \\
\text { USCE } \\
\text { USCE } \\
\text { USCE } \\
\text { USCE }\end{array}$ & $\begin{array}{l}23 I \\
23 J \\
23 I \\
23 D \\
23 E\end{array}$ & $\begin{array}{l}330 M S S P \\
211 \text { GORD } \\
211 \text { EUTWR } \\
211 \text { EUTWR } \\
211 \text { EUTWR }\end{array}$ & 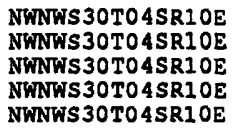 & $\begin{array}{l}10 / 07 / 1971 \\
12 / 02 / 1971 \\
12 / 08 / 1971 \\
08 / 31 / 1971 \\
09 / 30 / 1971\end{array}$ & $\begin{array}{l}587 \\
587 \\
563 \\
590 \\
585\end{array}$ & $\begin{array}{r}492 \\
380 \\
126 \\
145 \\
92\end{array}$ & $\begin{array}{l}2 \\
4 \\
8 \\
4 \\
2\end{array}$ & $\begin{array}{l}60 \\
60 \\
20 \\
--\end{array}$ \\
\hline $\begin{array}{l}\text { GO38 } \\
\text { GO40 } \\
\text { G041 } \\
\text { GO42 } \\
\text { G079 }\end{array}$ & $\begin{array}{l}\text { USCE } \\
\text { USCE } \\
\text { USCE } \\
\text { USCE } \\
\text { USCE }\end{array}$ & $\begin{array}{l}22 A \\
22 B \\
23 N \\
230 \\
6 D P 163\end{array}$ & $\begin{array}{l}211 \text { GORD } \\
211 E U T W R \\
211 \text { MCSN } \\
211 \text { EUTN } \\
211 \text { GORD }\end{array}$ & $\begin{array}{l}\text { SWNWS25TO 4SRO 9E } \\
\text { NWSWS 25T0 4SRO 9E } \\
\text { NWNWS 3OTO 4SRI OE } \\
\text { NWNWS 3OTO 4SRI OE } \\
\text { SESES 24TO 4SRO 9E }\end{array}$ & $\begin{array}{l}01 / 31 / 1972 \\
02 / 04 / 1972 \\
01 / 24 / 1972 \\
02 / 01 / 1972 \\
02 / 20 / 1973\end{array}$ & $\begin{array}{l}625 \\
625 \\
600 \\
561 \\
573\end{array}$ & $\begin{array}{r}360 \\
240 \\
200 \\
60 \\
222\end{array}$ & $\begin{array}{l}4 \\
4 \\
4 \\
6 \\
4\end{array}$ & $\begin{array}{l}-- \\
20 \\
9.0\end{array}$ \\
\hline $\begin{array}{l}\text { GO80 } \\
\text { G083 } \\
\text { G085 } \\
\text { G086 } \\
\text { G087 }\end{array}$ & $\begin{array}{l}\text { USCE } \\
\text { USCE } \\
\text { USCE } \\
\text { USCE } \\
\text { USCE }\end{array}$ & $\begin{array}{l}\text { 6DP1 } 64 \\
\text { NE1-1 } \\
\text { NE1-3 } \\
\text { MEI-1 } \\
M E 1-2\end{array}$ & $\begin{array}{l}211 \text { EUTW } \\
211 \text { GORD } \\
211 \text { EUTW } \\
211 \text { GORD } \\
211 \text { EUTWL }\end{array}$ & $\begin{array}{l}\text { SESES24TO 4SRO 9E } \\
\text { NENWSO5TO } 4 \text { SR1OE } \\
\text { NENWSO5TO 4SR1OE } \\
\text { NENES21TO } 4 \text { SR1OE } \\
\text { NENES21TO4SRIOE }\end{array}$ & $\begin{array}{l}02 / 23 / 1973 \\
10 / 17 / 1977 \\
06 / 22 / 1978 \\
02 / 08 / 1979 \\
02 / 13 / 1979\end{array}$ & $\begin{array}{l}572.60 \\
500 \\
495 \\
560 \\
560\end{array}$ & $\begin{array}{r}125 \\
190 \\
65 \\
204 \\
129\end{array}$ & $\begin{array}{l}4 \\
3 \\
3 \\
1.50 \\
1.50\end{array}$ & $\begin{array}{l}-- \\
-- \\
-- \\
--\end{array}$ \\
\hline $\begin{array}{l}\text { G092 } \\
\text { G093 } \\
\text { G095 } \\
\text { G100 } \\
\text { G102 }\end{array}$ & $\begin{array}{l}\text { USCE } \\
\text { USCE } \\
\text { USCE } \\
\text { USCE } \\
\text { USCE }\end{array}$ & $\begin{array}{l}M E 2-1 \\
M E 2-2 \\
M E 3-2 \\
S E 1-2 \\
S E 1-4\end{array}$ & $\begin{array}{l}211 \text { GORD } \\
211 \text { EUTW } \\
211 \text { GORD } \\
211 \text { GORD } \\
\text { 211EUTW }\end{array}$ & $\begin{array}{l}\text { NWSWS1 4TO 4SRIOE } \\
\text { NWSWS1 4TO 4SRIOE } \\
\text { SWNES13TO 4SRIOE } \\
\text { SWNWS35TO 4SR1 OE } \\
\text { SWNWS 35TO4SR1OE }\end{array}$ & $\begin{array}{l}02 / 16 / 1979 \\
02 / 16 / 1979 \\
08 / 28 / 1979 \\
06 / 08 / 1979 \\
04 / 30 / 1979\end{array}$ & $\begin{array}{l}560 \\
560 \\
517 \\
560 \\
560\end{array}$ & $\begin{array}{r}162 \\
67 \\
93 \\
218 \\
73\end{array}$ & $\begin{array}{l}1.50 \\
1.50 \\
1.50 \\
1.50 \\
1.50\end{array}$ & $\begin{array}{l}=- \\
-- \\
--\end{array}$ \\
\hline $\begin{array}{l}\text { G104 } \\
\text { G106 } \\
\text { G112 } \\
\text { G113 } \\
\text { G116 }\end{array}$ & $\begin{array}{l}\text { USCE } \\
\text { USCE } \\
\text { USCE } \\
\text { USCE } \\
\text { USCE }\end{array}$ & $\begin{array}{l}\text { SE2-2 } \\
\text { SE2-4 } \\
\text { 2DP147 } \\
\text { 2DP148 } \\
\text { 4DP156 }\end{array}$ & $\begin{array}{l}\text { 211GORD } \\
211 \text { EUTW } \\
211 \text { GORD } \\
211 \text { EUTW } \\
\text { 211GORD }\end{array}$ & $\begin{array}{l}\text { NWSWS 25TO 4SR1 OE } \\
\text { NWSWS 25TO 4SRI OE } \\
\text { SESWS11TO4SRO 9E } \\
\text { SESWS11TO 4SRO 9E } \\
\text { SESWS31TO4SRI OE }\end{array}$ & $\begin{array}{l}05 / 17 / 1979 \\
05 / 24 / 1979 \\
07 / 20 / 1972 \\
07 / 25 / 1972 \\
01 / 01 / 1973\end{array}$ & $\begin{array}{l}580 \\
580 \\
504.50 \\
504.70 \\
490.10\end{array}$ & $\begin{array}{l}183 \\
103 \\
165 \\
130 \\
153\end{array}$ & $\begin{array}{l}1.50 \\
1.50 \\
3 \\
3 \\
3.50\end{array}$ & $\begin{array}{l}-- \\
-- \\
-- \\
--\end{array}$ \\
\hline $\begin{array}{l}\text { G118 } \\
\text { G121 } \\
\text { G122 } \\
\text { JOOB } \\
\text { JO13 }\end{array}$ & $\begin{array}{l}\text { USCE } \\
\text { USCE } \\
\text { USCE } \\
\text { USCE } \\
\text { USCE }\end{array}$ & $\begin{array}{l}\text { 4DP158 } \\
\text { 9DP173 } \\
\text { 9DP174 } \\
33 A \\
34 A\end{array}$ & $\begin{array}{l}\text { 211EUTW } \\
211 \text { GORD } \\
211 \text { EUTW } \\
211 \text { EUTWL } \\
211 \text { GORD }\end{array}$ & $\begin{array}{l}\text { SESWS 31TO4SR1 OE } \\
\text { NESES14TO4SRO 9E } \\
\text { NESES14TO4SRO 9E } \\
\text { SENESO6TO5SRO 9E } \\
\text { NENWSO5TO5SR1 OE }\end{array}$ & $\begin{array}{l}10 / 26 / 1972 \\
04 / 19 / 1973 \\
04 / 23 / 1973 \\
07 /--/ 1971 \\
07 /--/ 1971\end{array}$ & $\begin{array}{l}487.70 \\
552.20 \\
552.40 \\
515 \\
560\end{array}$ & $\begin{array}{r}56 \\
220 \\
163 \\
172 \\
266\end{array}$ & $\begin{array}{l}4 \\
4 \\
4 \\
4 \\
4\end{array}$ & $\begin{array}{l}-- \\
-- \\
-- \\
8.0\end{array}$ \\
\hline $\begin{array}{l}\text { JO14 } \\
\text { JO16 } \\
\text { JO17 } \\
\text { JO18 } \\
\text { JO19 }\end{array}$ & $\begin{array}{l}\text { USCE } \\
\text { USCE } \\
\text { USCE } \\
\text { USCE } \\
\text { USCE }\end{array}$ & $\begin{array}{l}34 \mathrm{~B} \\
33 \mathrm{~B} \\
33 \mathrm{C} \\
31 \mathrm{~A} \\
31 \mathrm{~B}\end{array}$ & $\begin{array}{l}\text { 211EUTWR } \\
211 \text { EUTWR } \\
211 \text { GORD } \\
211 \text { GORD } \\
\text { 211EUTWR }\end{array}$ & $\begin{array}{l}\text { NENWSO5TO5SR1 OE } \\
\text { SENESO6T05SR09E } \\
\text { SENESO6T05SRO9E } \\
\text { SWSESO1TO5SRO 9E } \\
\text { SWSESO1T05SRO 9E }\end{array}$ & $\begin{array}{l}07 /--11971 \\
07 /--/ 1971 \\
09 / 01 / 1971 \\
03 / 15 / 1972 \\
03 / 27 / 1972\end{array}$ & $\begin{array}{l}560 \\
515 \\
515 \\
473 \\
473\end{array}$ & $\begin{array}{r}134 \\
90 \\
212 \\
178 \\
74\end{array}$ & $\begin{array}{l}4 \\
4 \\
2 \\
4 \\
4\end{array}$ & $\begin{array}{l}8.0 \\
-- \\
1.0 \\
--\end{array}$ \\
\hline $\begin{array}{l}\text { JO20 } \\
\text { JO21 } \\
\text { JO66 } \\
\text { JO } 75 \\
\text { JO } 76\end{array}$ & $\begin{array}{l}\text { USCE } \\
\text { USCE } \\
\text { USCE } \\
\text { USCE } \\
\text { USCE }\end{array}$ & $\begin{array}{l}32 A \\
32 B \\
\text { SW1-2 } \\
\text { SW1-1 } \\
\text { 5DP159 }\end{array}$ & $\begin{array}{l}211 \text { GORD } \\
211 \text { EUTWR } \\
211 \text { EUTW } \\
211 \text { GORD } \\
211 \text { GORD }\end{array}$ & $\begin{array}{l}\text { NWNWSO6TO5SR10E } \\
\text { NWNWSO6TO5SR1OE } \\
\text { NWNWS12T05SRO9E } \\
\text { SWNWS12T05SRO9E } \\
\text { NESWSOBTO5SR1OE }\end{array}$ & $\begin{array}{l}04 / 27 / 1972 \\
04 /-1 / 1972 \\
09 / 01 / 1977 \\
08 / 25 / 1977 \\
11 / 06 / 1972\end{array}$ & $\begin{array}{l}530 \\
530 \\
560 \\
560 \\
437.20\end{array}$ & $\begin{array}{l}240 \\
112 \\
150 \\
264 \\
148\end{array}$ & $\begin{array}{l}4 \\
4 \\
1.50 \\
1.50 \\
4\end{array}$ & $\begin{array}{c}10 \\
8.0 \\
-- \\
--\end{array}$ \\
\hline $\begin{array}{l}\text { J077 } \\
\text { JO80 } \\
\text { J081 } \\
\text { L029 } \\
\text { L030 }\end{array}$ & $\begin{array}{l}\text { USCE } \\
\text { USCE } \\
\text { USCE } \\
\text { USCE } \\
\text { USCE }\end{array}$ & $\begin{array}{l}5 D P 160 \\
E 1-2 \\
E 1-3 \\
54 A \\
55 A\end{array}$ & $\begin{array}{l}\text { 211EUTW } \\
211 \text { GORD } \\
211 \text { EUTW } \\
211 \text { GORD } \\
211 \text { GORD }\end{array}$ & $\begin{array}{l}\text { NESWSO8TO5SR1OE } \\
\text { SWSESO9TO5SRIOE } \\
\text { SWSESO 9TO5SRIOE } \\
\text { SWSWS11T07SRO 9E } \\
\text { SESES11TO7SRO 9E }\end{array}$ & $\begin{array}{l}11 / 08 / 1972 \\
02 / 16 / 1981 \\
03 / 16 / 1981 \\
04 / 28 / 1972 \\
05 / 11 / 1972\end{array}$ & $\begin{array}{l}436 \\
520 \\
520 \\
332 \\
380\end{array}$ & $\begin{array}{r}60 \\
131 \\
102 \\
27 \\
50\end{array}$ & $\begin{array}{l}3 \\
1.50 \\
1.50 \\
4 \\
4\end{array}$ & $\begin{array}{l}-- \\
-- \\
-- \\
18\end{array}$ \\
\hline $\begin{array}{l}2031 \\
L 032 \\
L 033 \\
L 034 \\
L 051\end{array}$ & $\begin{array}{l}\text { USCE } \\
\text { USCE } \\
\text { USCE } \\
\text { USCE } \\
\text { USCE }\end{array}$ & $\begin{array}{l}45 A \\
45 B \\
54 B \\
54 C \\
\text { GW125 }\end{array}$ & $\begin{array}{l}211 G O R D \\
211 \mathrm{MCSN} \\
110 \mathrm{ALVM} \\
110 \mathrm{ALVM} \\
110 \mathrm{ALVM}\end{array}$ & $\begin{array}{l}\text { NWSWS25T06SRO9E } \\
\text { SWNWS 25TO6SRO9E } \\
\text { SWSWS11TO7SRO9E } \\
\text { SWSWS11TO7SRO9E } \\
\text { SENES02T07SRO9E }\end{array}$ & $\begin{array}{l}03 / 29 / 1972 \\
03 / 29 / 1972 \\
06 / 06 / 1972 \\
05 / 01 / 1972 \\
07 / 22 / 1975\end{array}$ & $\begin{array}{l}485 \\
485 \\
332 \\
333 \\
367.10\end{array}$ & $\begin{array}{l}92 \\
76 \\
12 \\
13 \\
17\end{array}$ & $\begin{array}{l}4 \\
4 \\
4 \\
2 \\
2\end{array}$ & $\begin{array}{l}-- \\
-- \\
0.50 \\
--\end{array}$ \\
\hline
\end{tabular}




\section{APPENDIX A}

\section{GROUND-WATER DATA}

\section{HYDROGRAPHS}

33

PAGE 35 FOLLOWS 


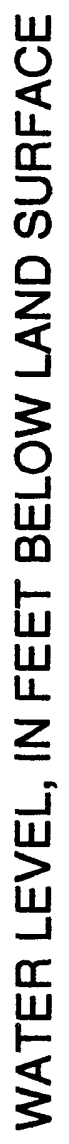
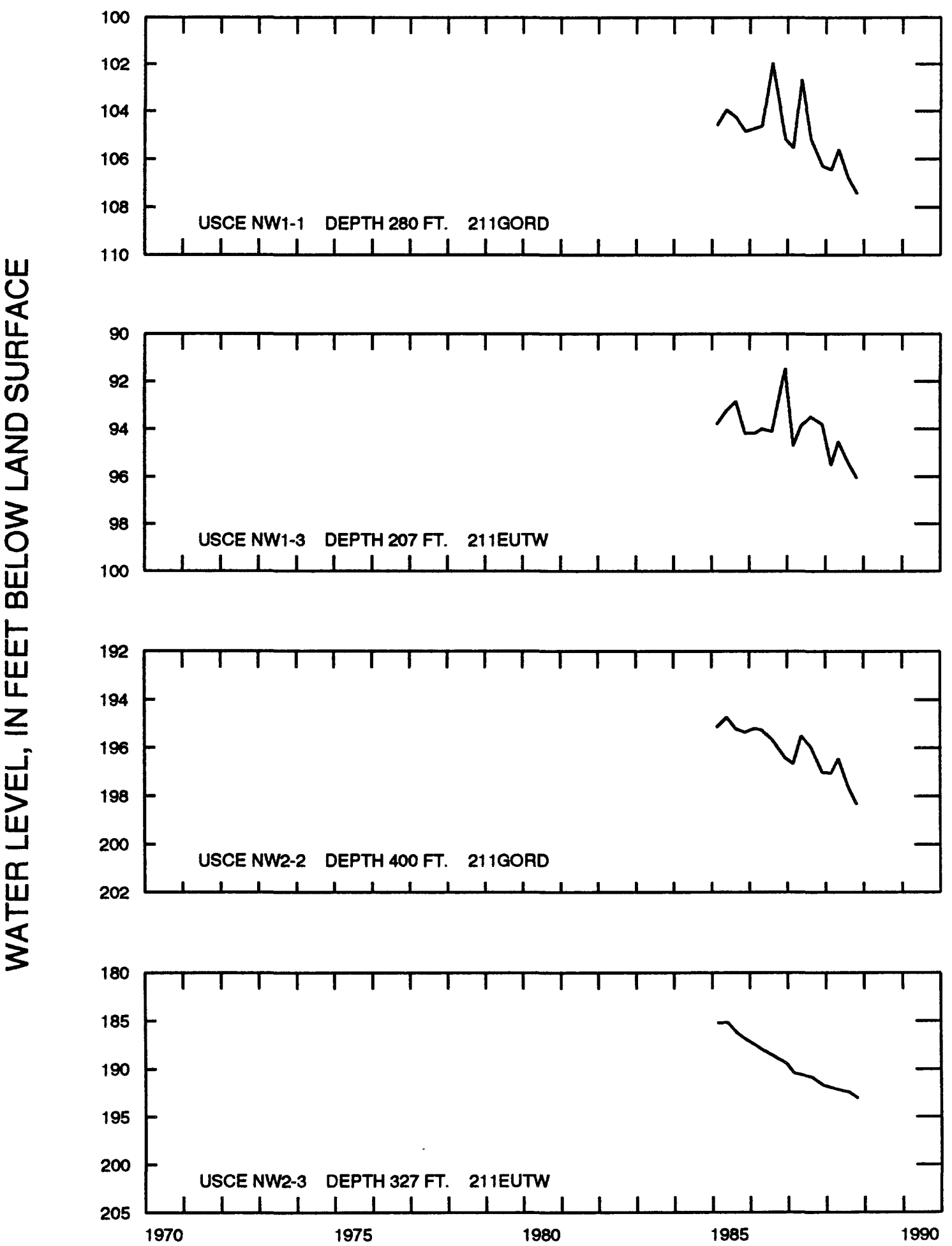

HYDROGRAPHS OF TENNESSEE-TOMBIGBEE OBSERVATION WELLS 


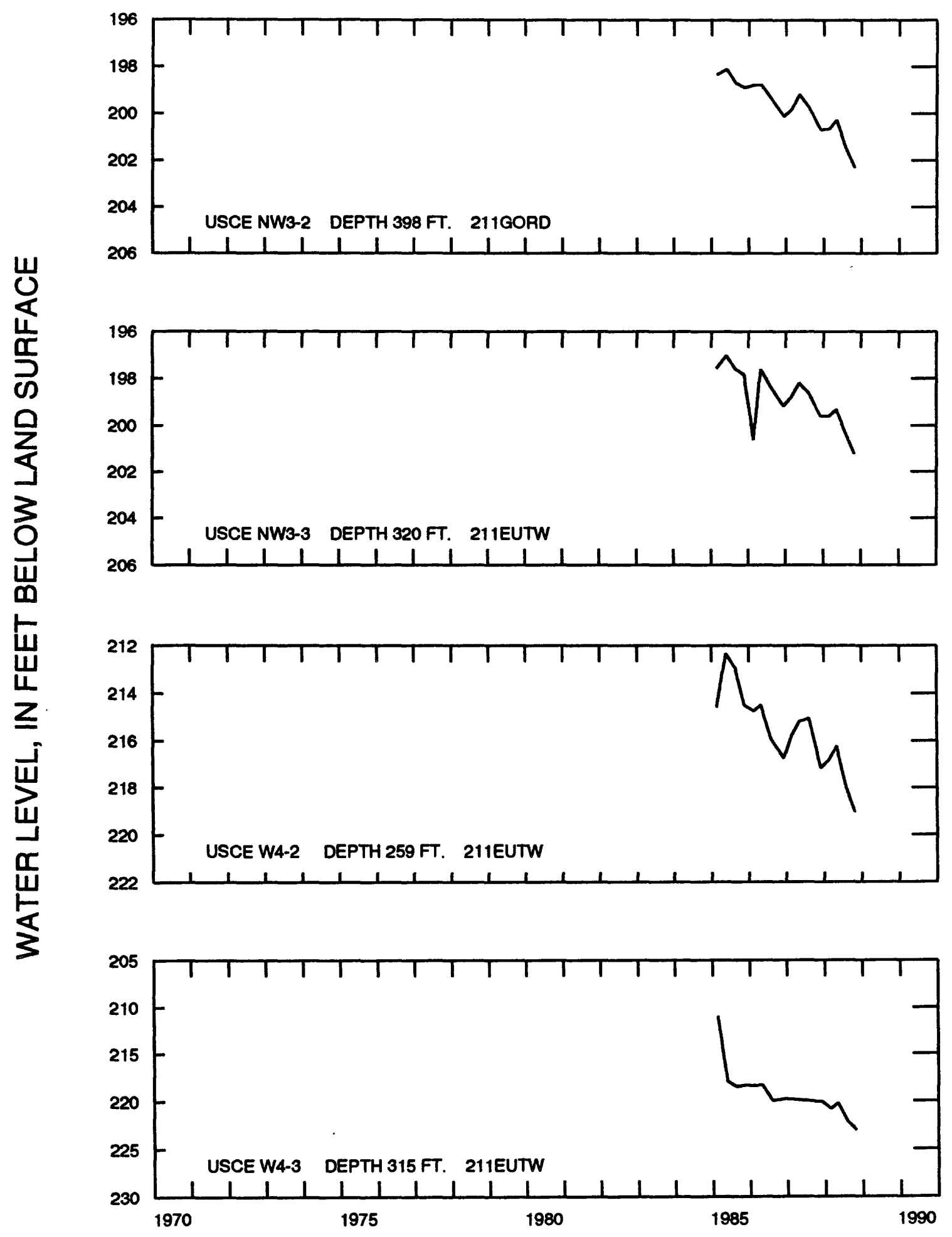

HYDROGRAPHS OF TENNESSEE-TOMBIGBEE OBSERVATION WELLS 


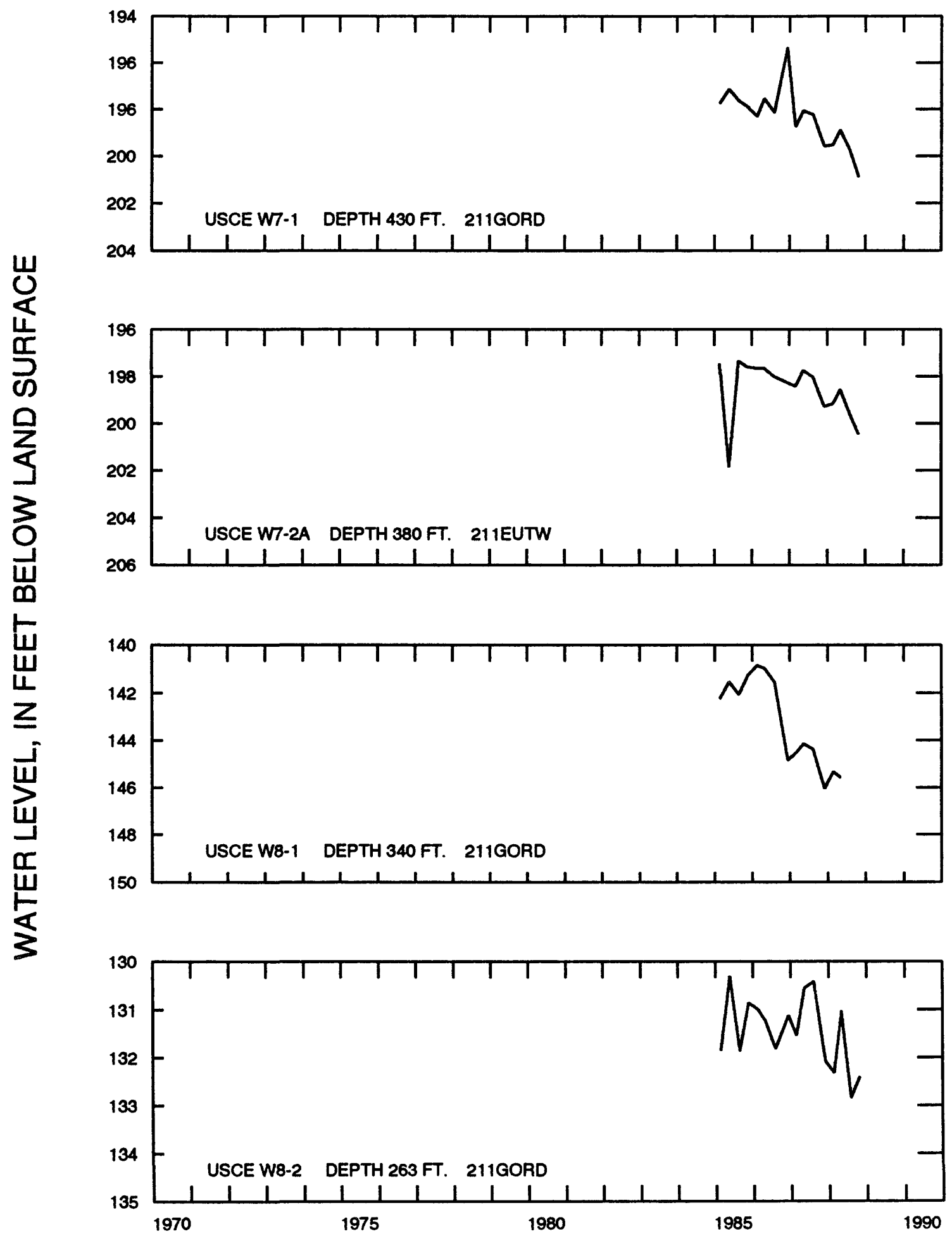

HYDROGRAPHS OF TENNESSEE-TOMBIGBEE OBSERVATION WELLS 


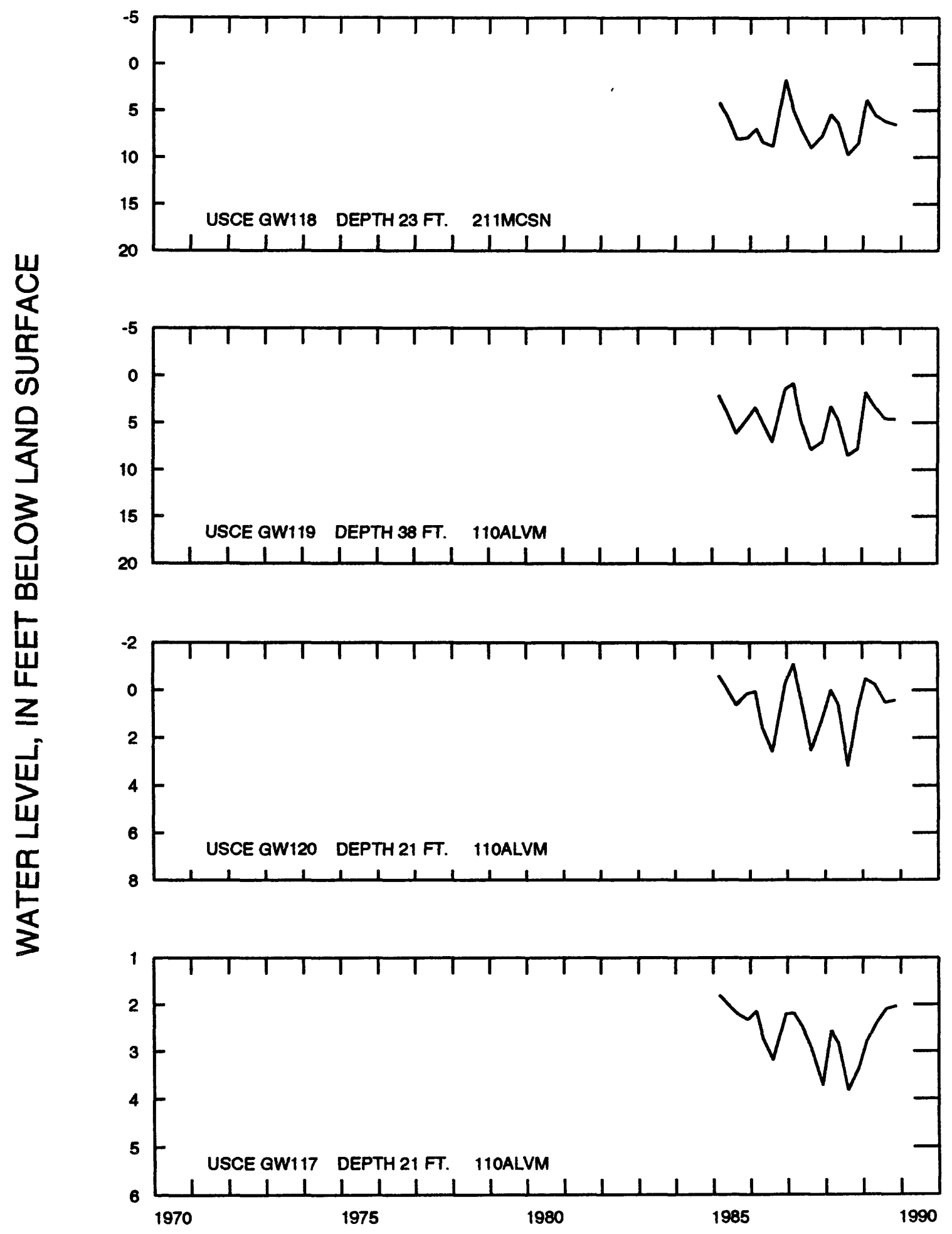

HYDROGRAPHS OF TENNESSEE-TOMBIGBEE OBSERVATION WELLS 


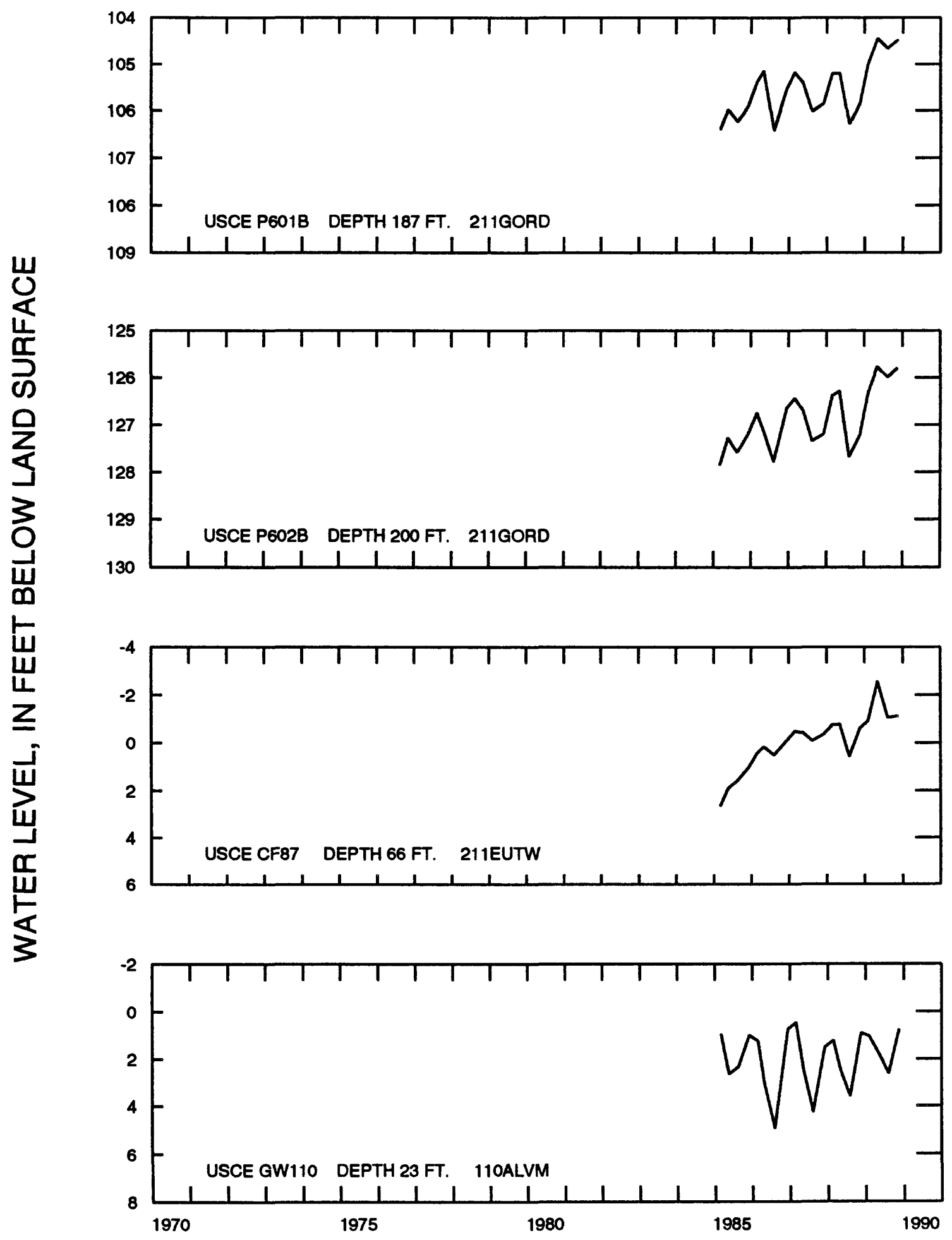

HYDROGRAPHS OF TENNESSEE-TOMBIGBEE OBSERVATION WELLS 


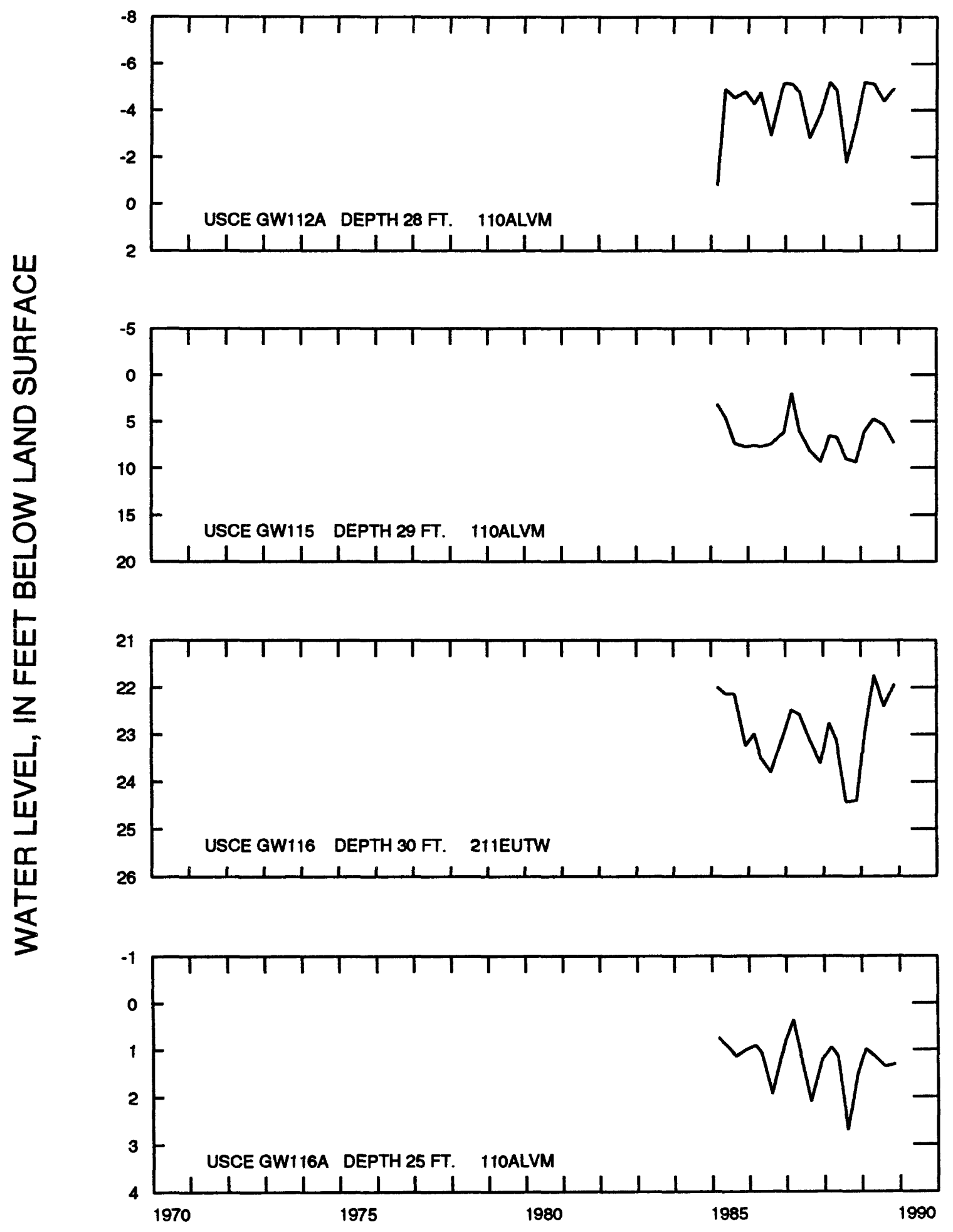

HYDROGRAPHS OF TENNESSEE-TOMBIGBEE OBSERVATION WELLS 


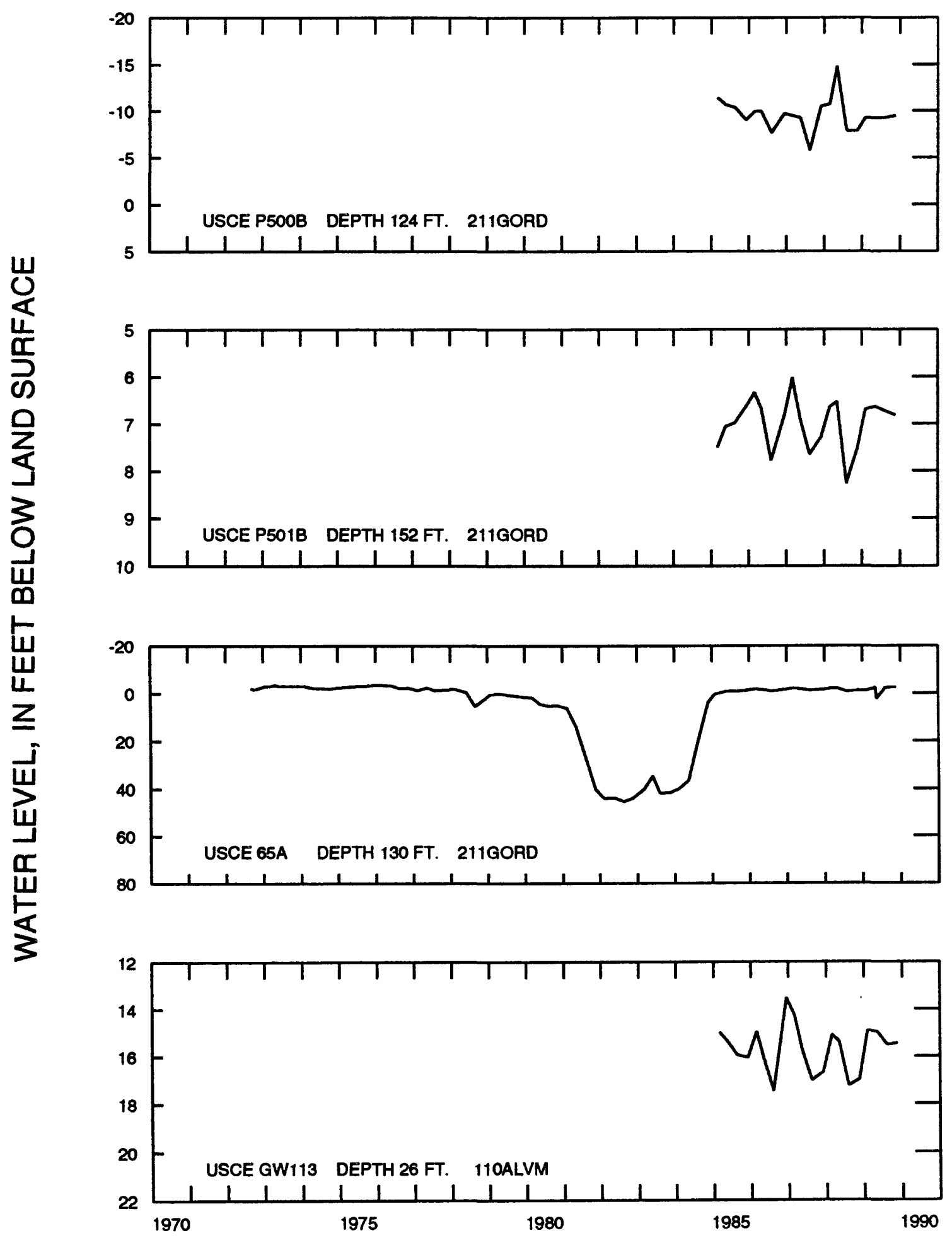

HYDROGRAPHS OF TENNESSEE-TOMBIGBEE OBSERVATION WELLS 


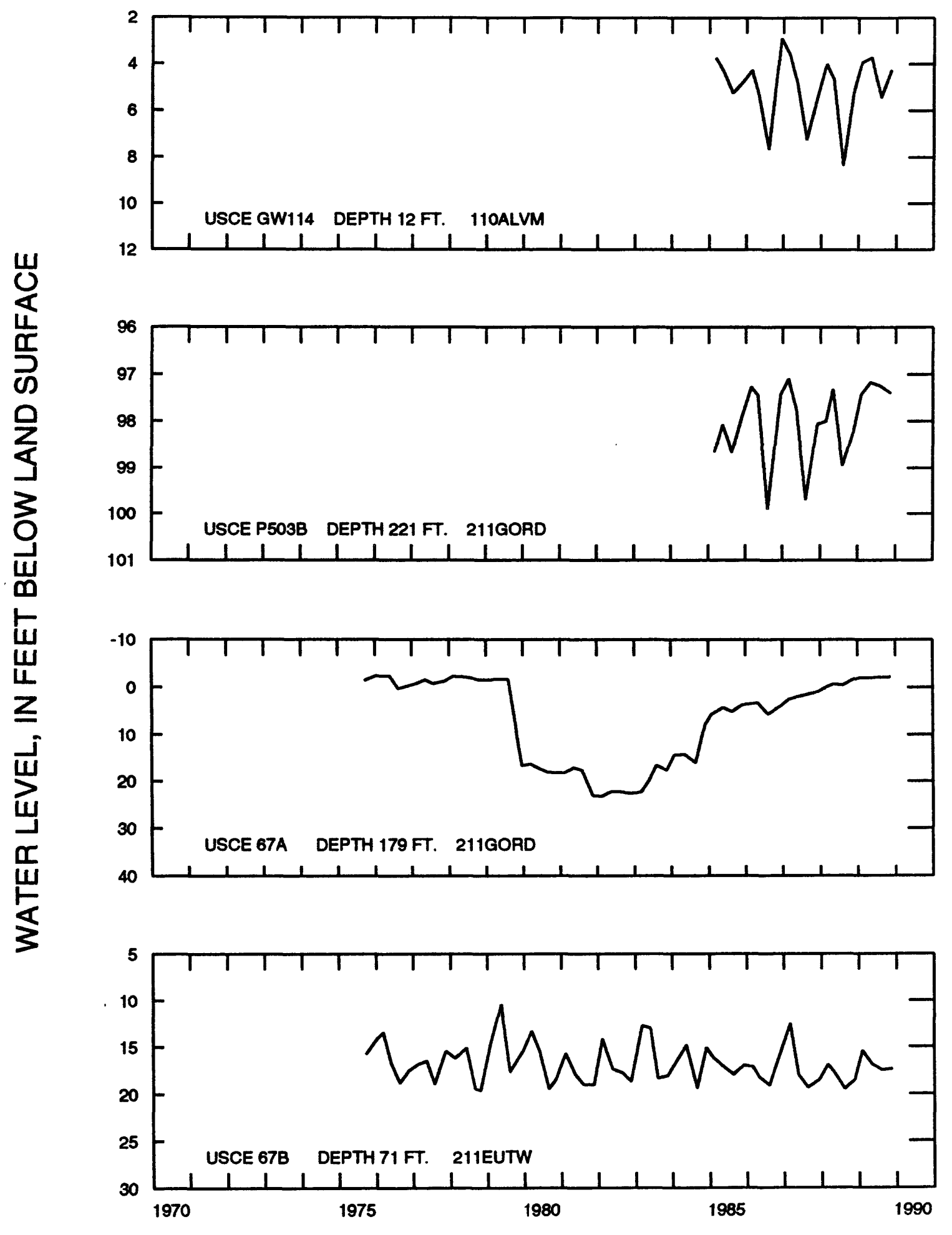

HYDROGRAPHS OF TENNESSEE-TOMBIGBEE OBSERVATION WELLS 


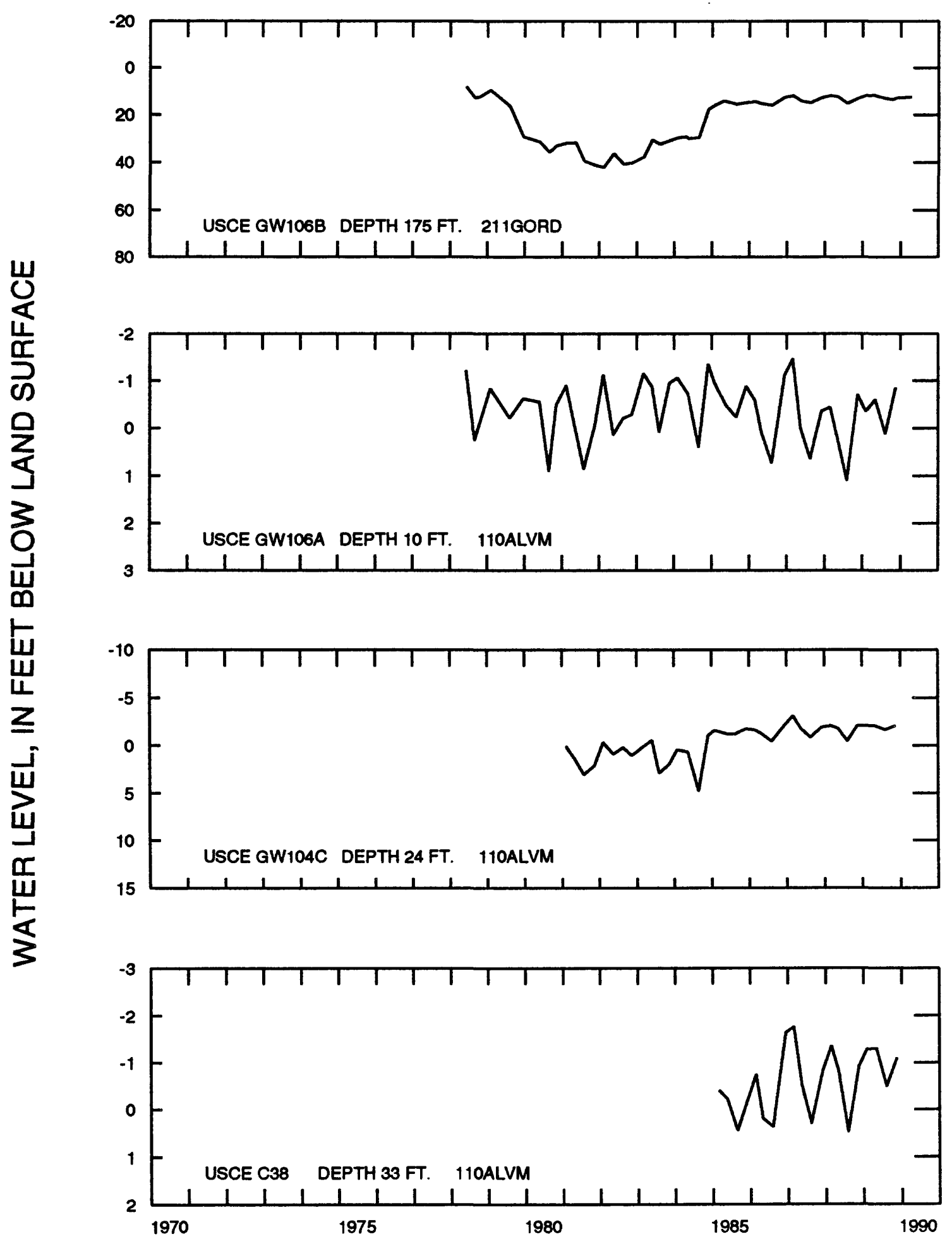

HYDROGRAPHS OF TENNESSEE-TOMBIGBEE OBSERVATION WELLS 


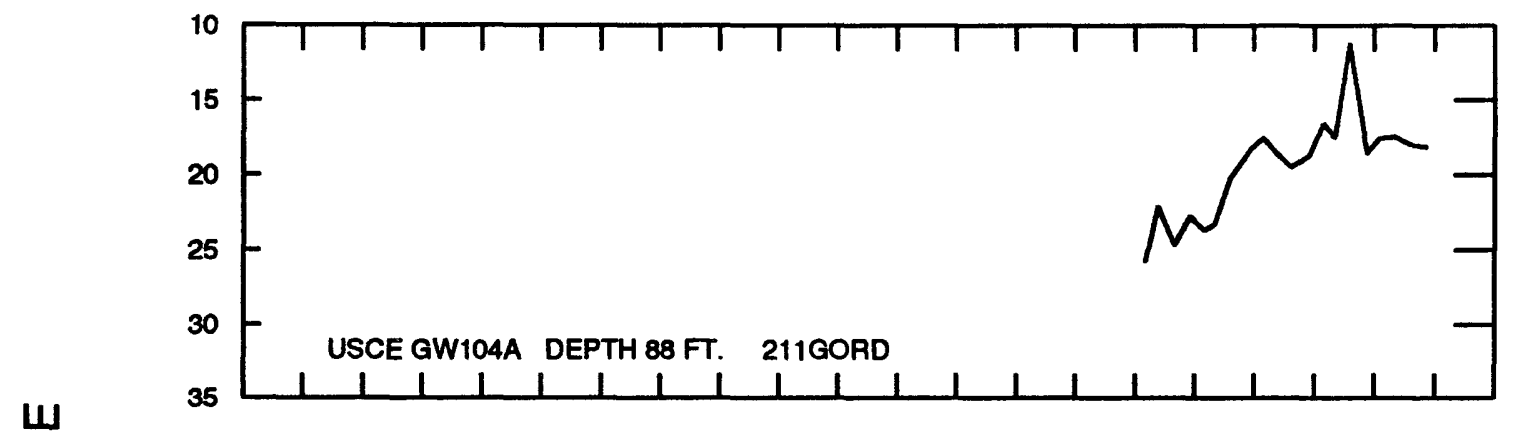

岀

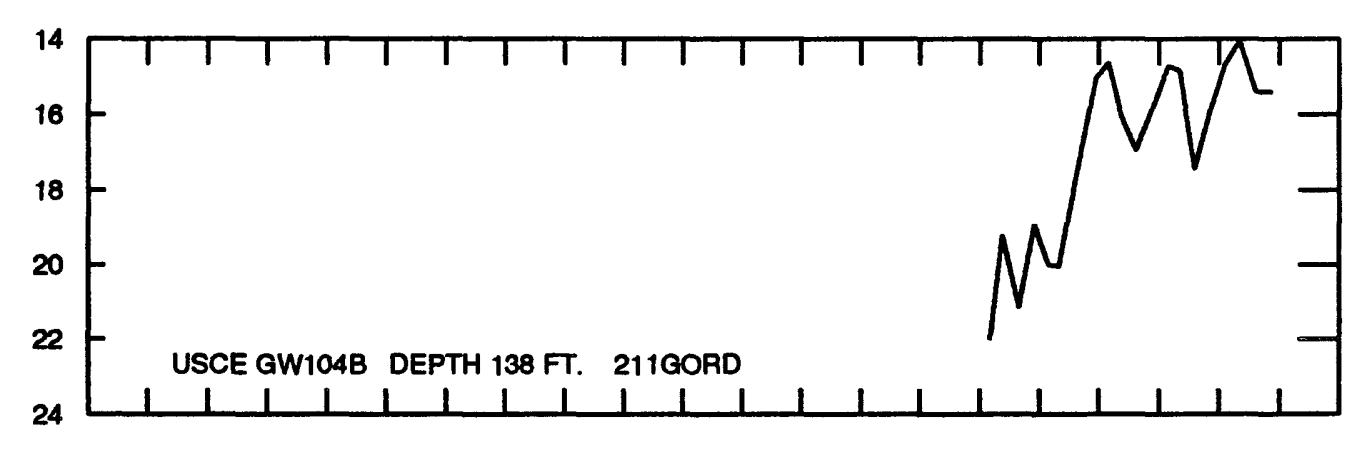

HYDROGRAPHS OF TENNESSEE-TOMBIGBEE OBSERVATION WELLS 


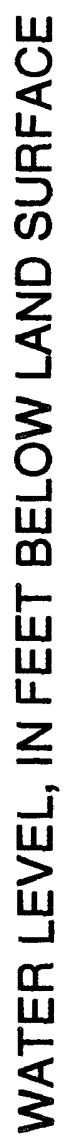
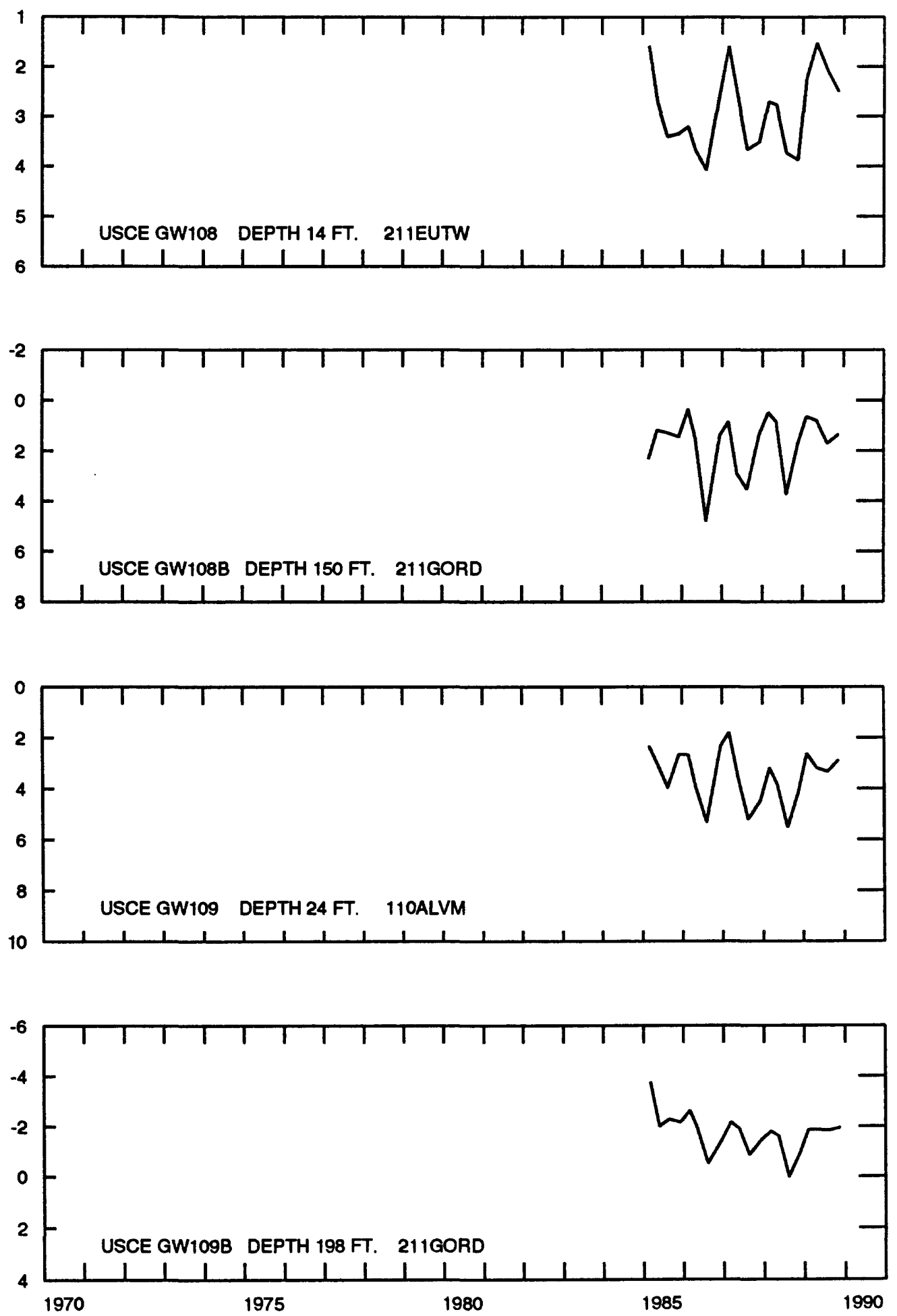

HYDROGRAPHS OF TENNESSEE-TOMBIGBEE OBSERVATION WELLS 


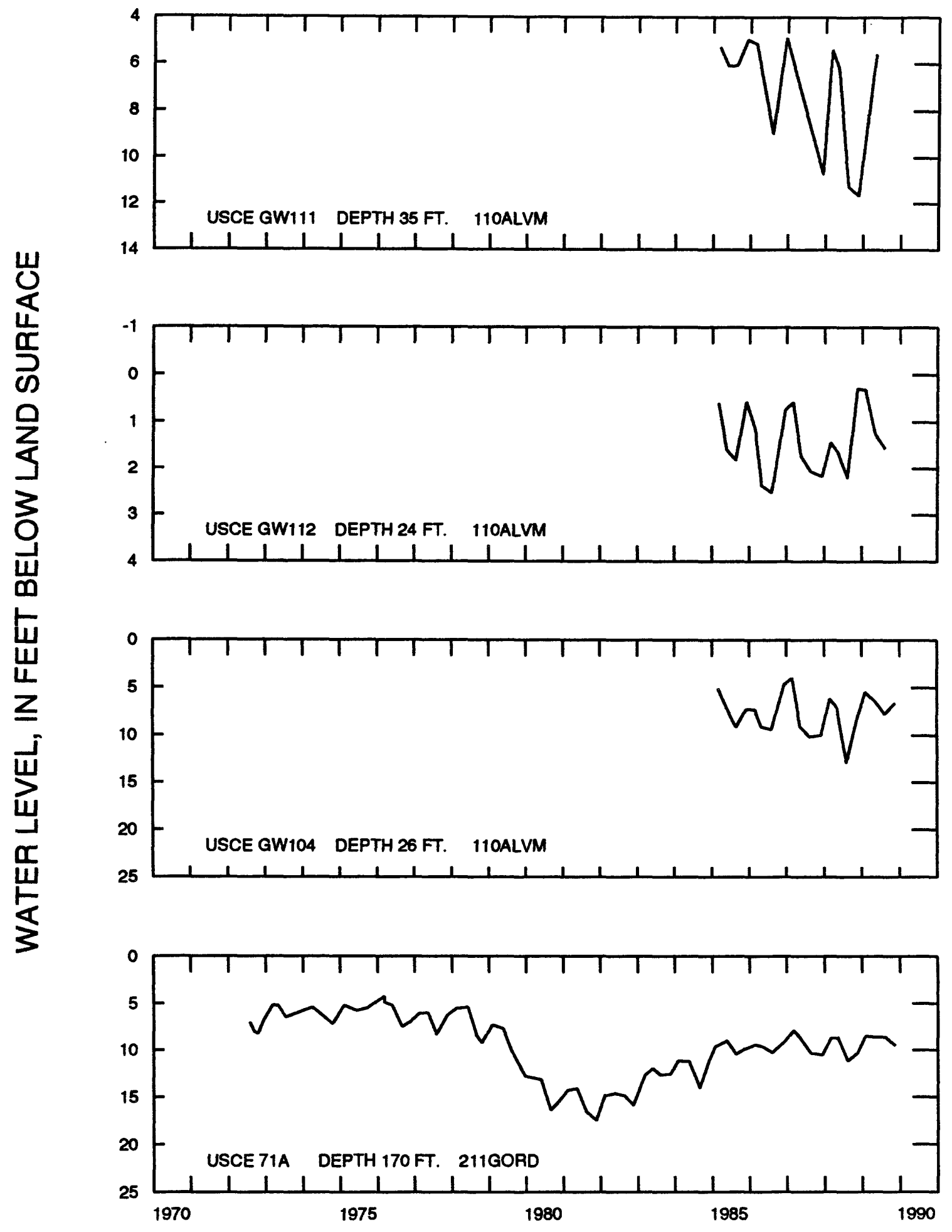

HYDROGRAPHS OF TENNESSEE-TOMBIGBEE OBSERVATION WELLS 


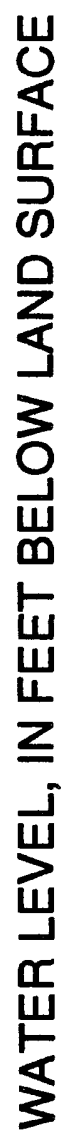
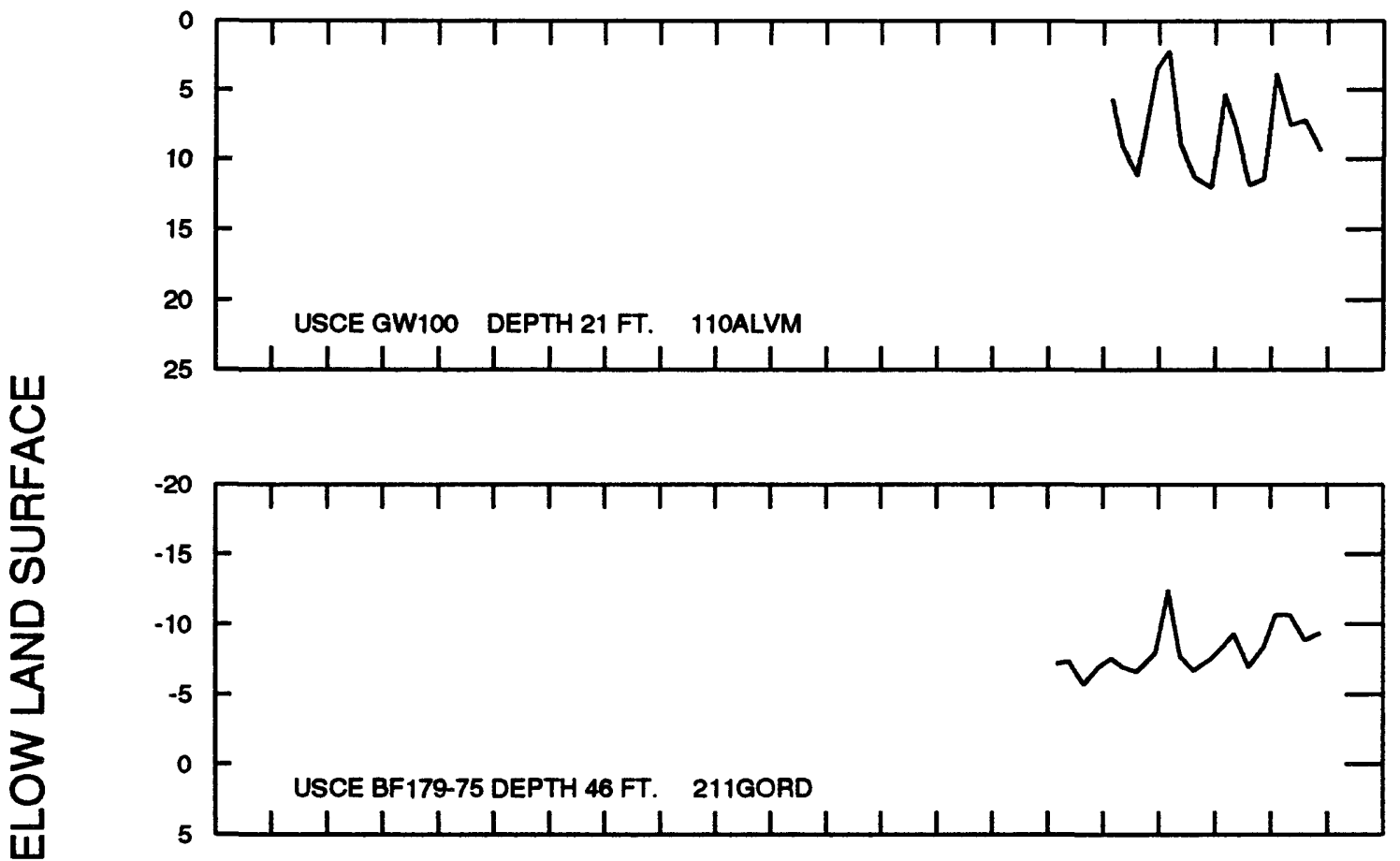

HYDROGRAPHS OF TENNESSEE-TOMBIGBEE OBSERVATION WELLS 


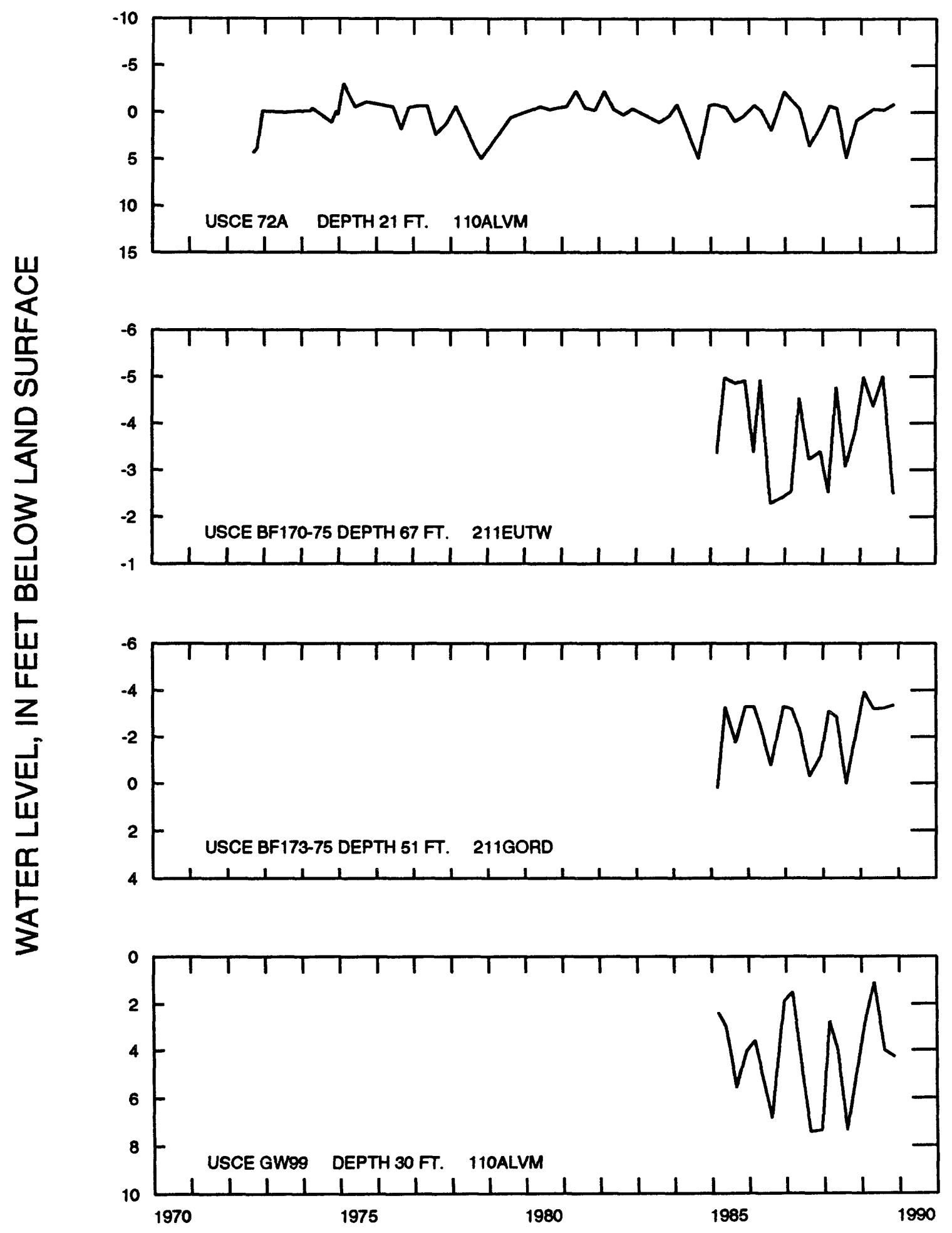

HYDROGRAPHS OF TENNESSEE-TOMBIGBEE OBSERVATION WELLS 


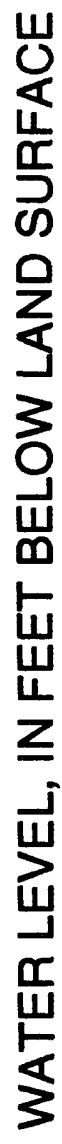
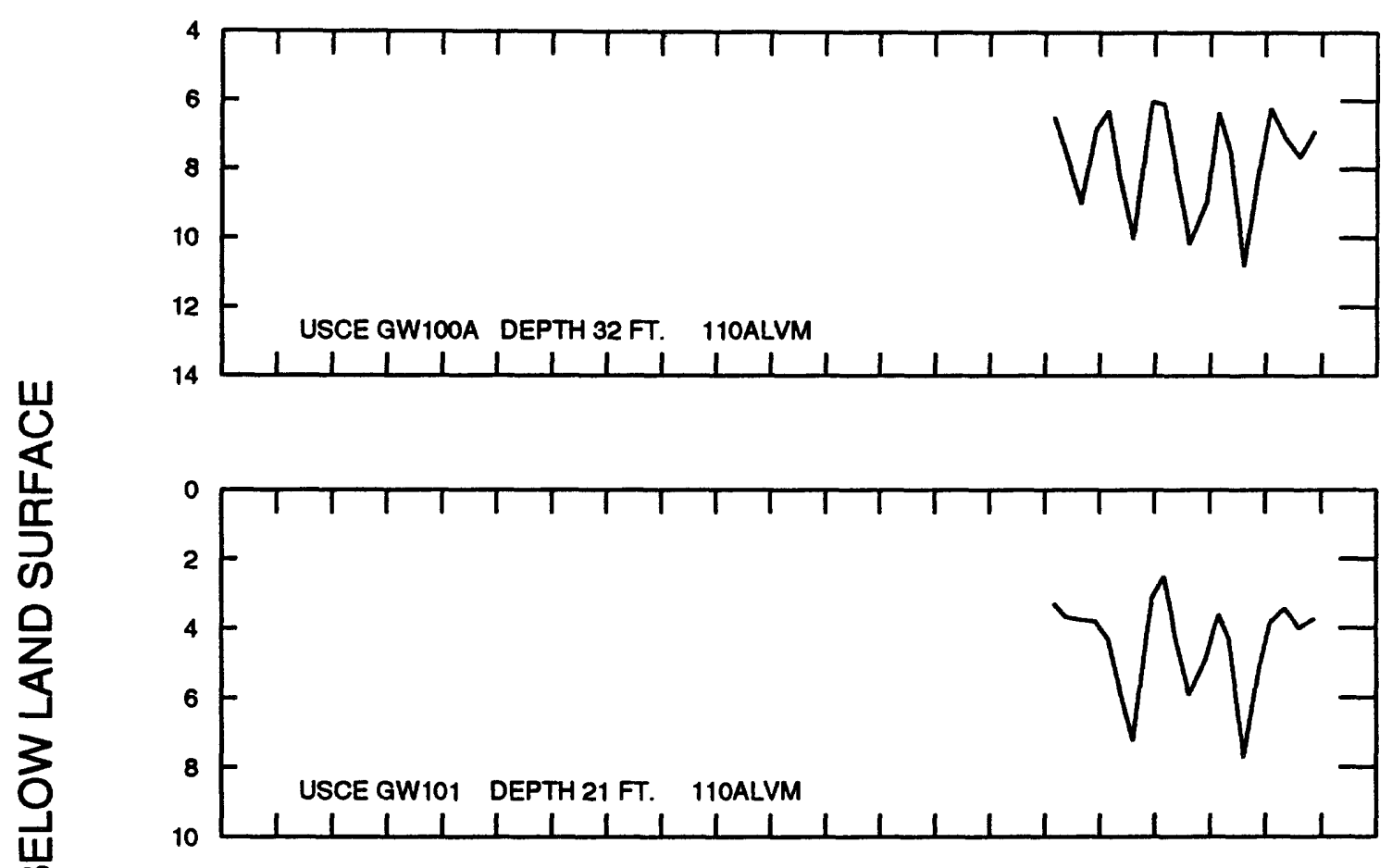

HYDROGRAPHS OF TENNESSEE-TOMBIGBEE OBSERVATION WELLS 


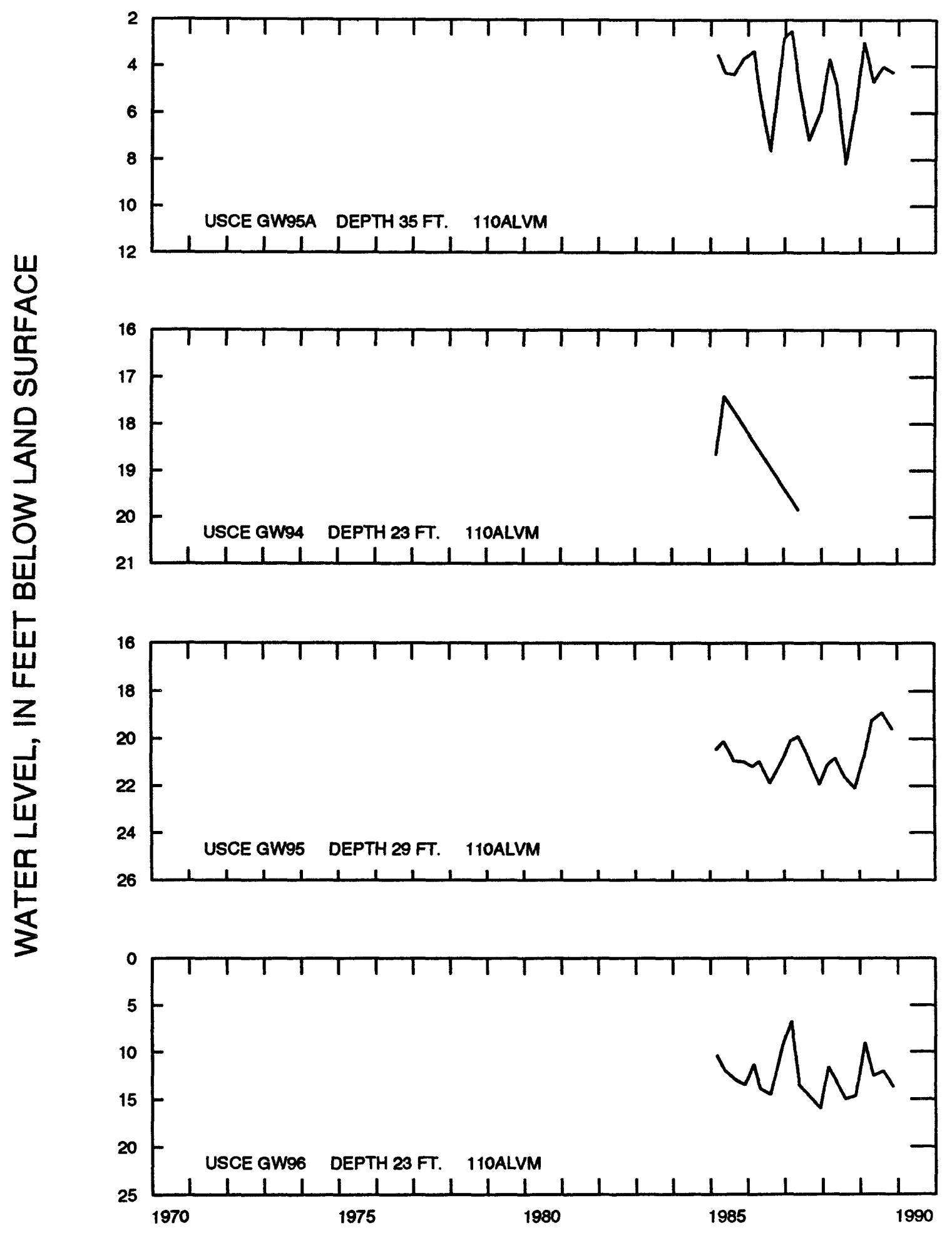

HYDROGRAPHS OF TENNESSEE-TOMBIGBEE OBSERVATION WELLS 
Ư
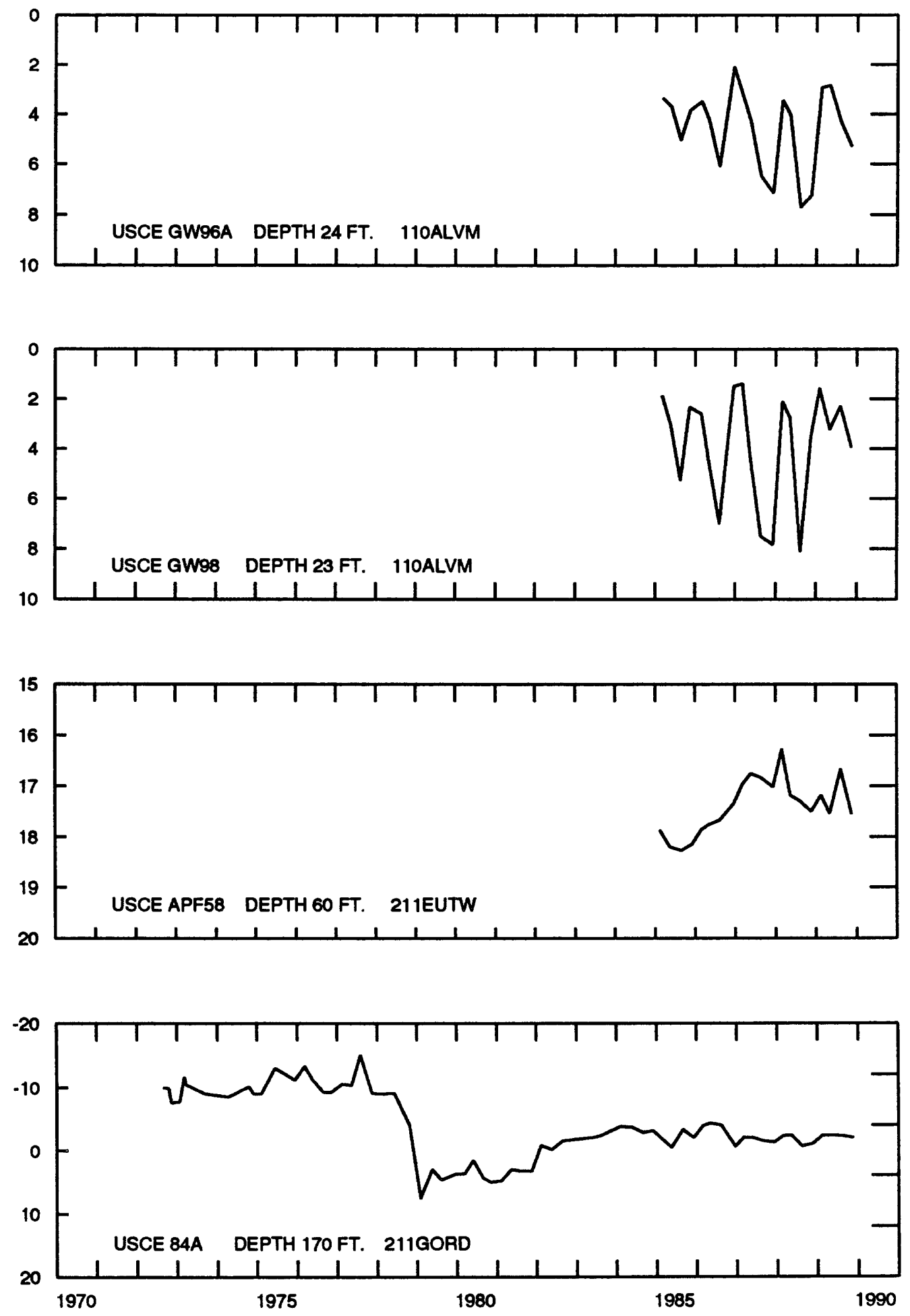

HYDROGRAPHS OF TENNESSEE-TOMBIGBEE OBSERVATION WELLS 


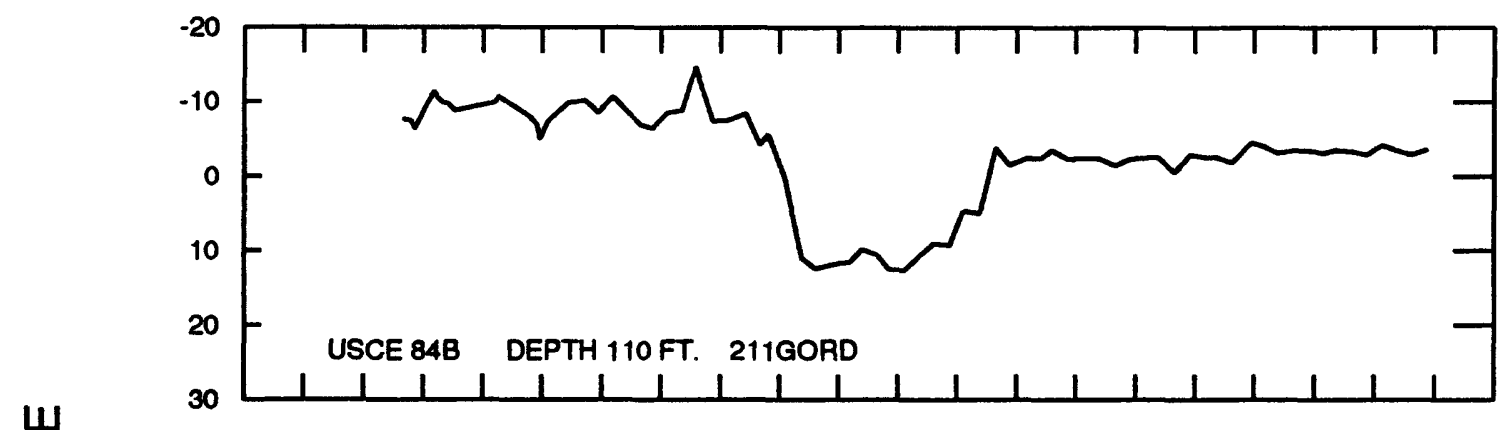

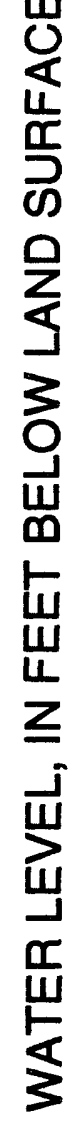

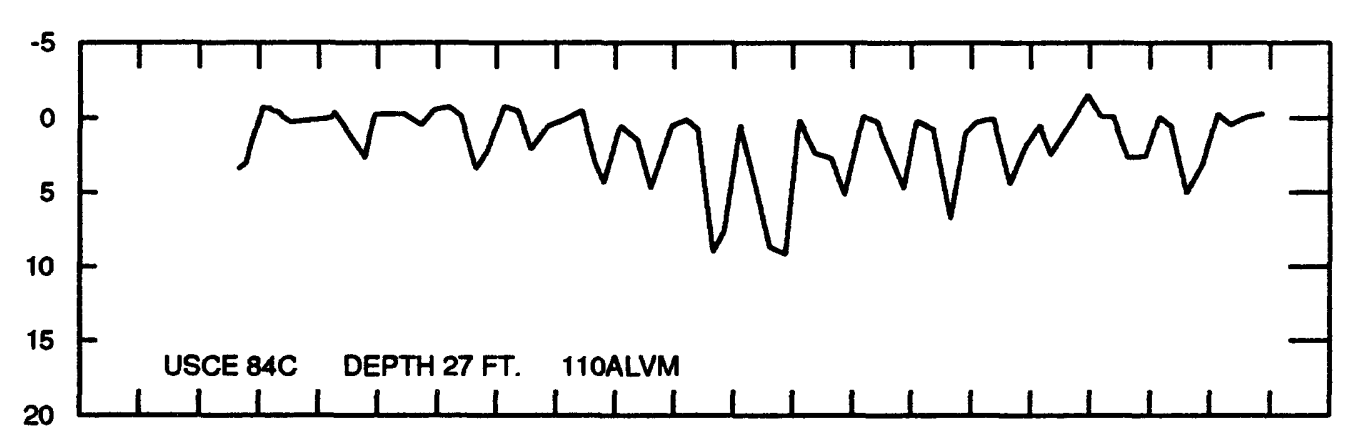

HYDROGRAPHS OF TENNESSEE-TOMBIGBEE OBSERVATION WELLS 


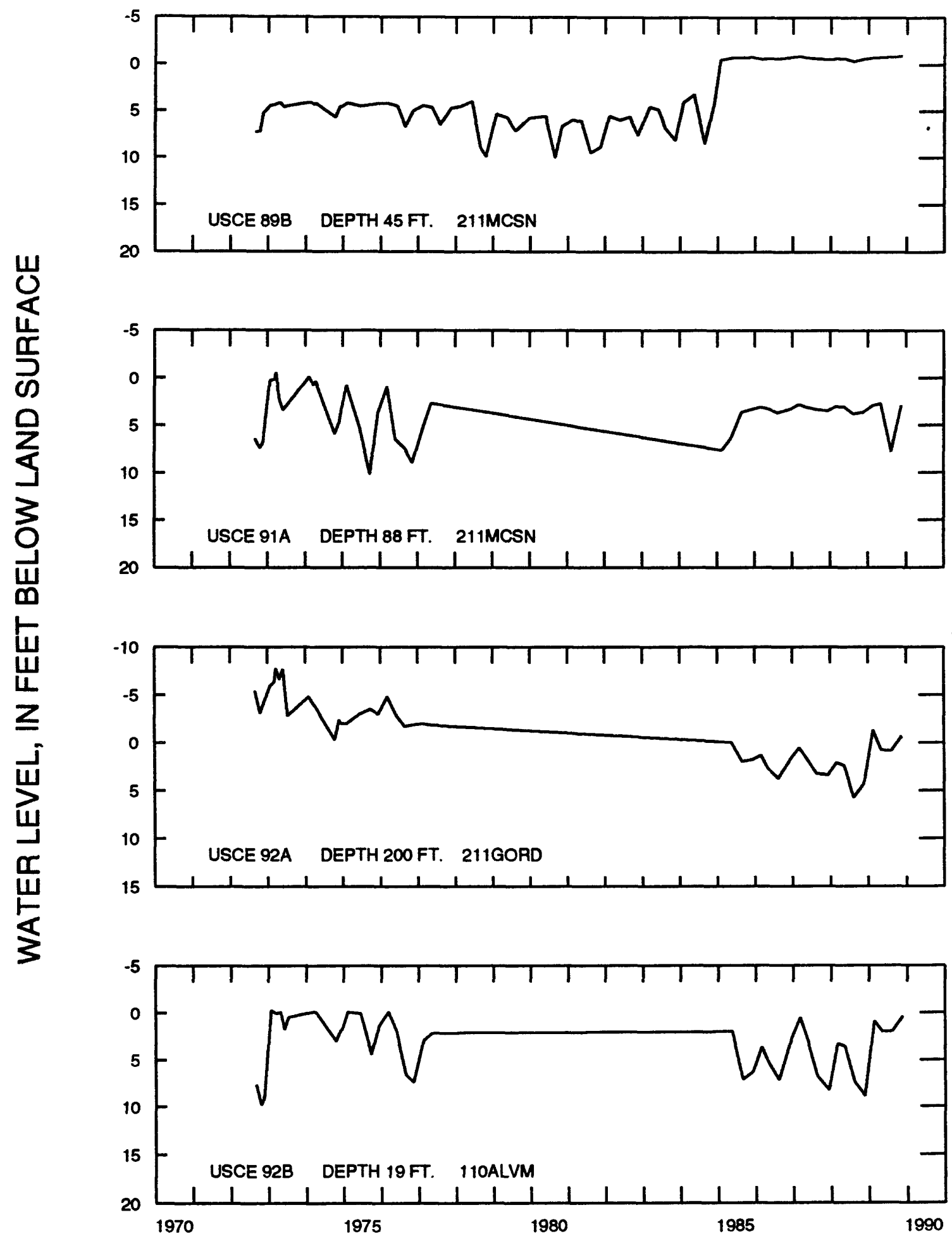

HYDROGRAPHS OF TENNESSEE-TOMBIGBEE OBSERVATION WELLS 


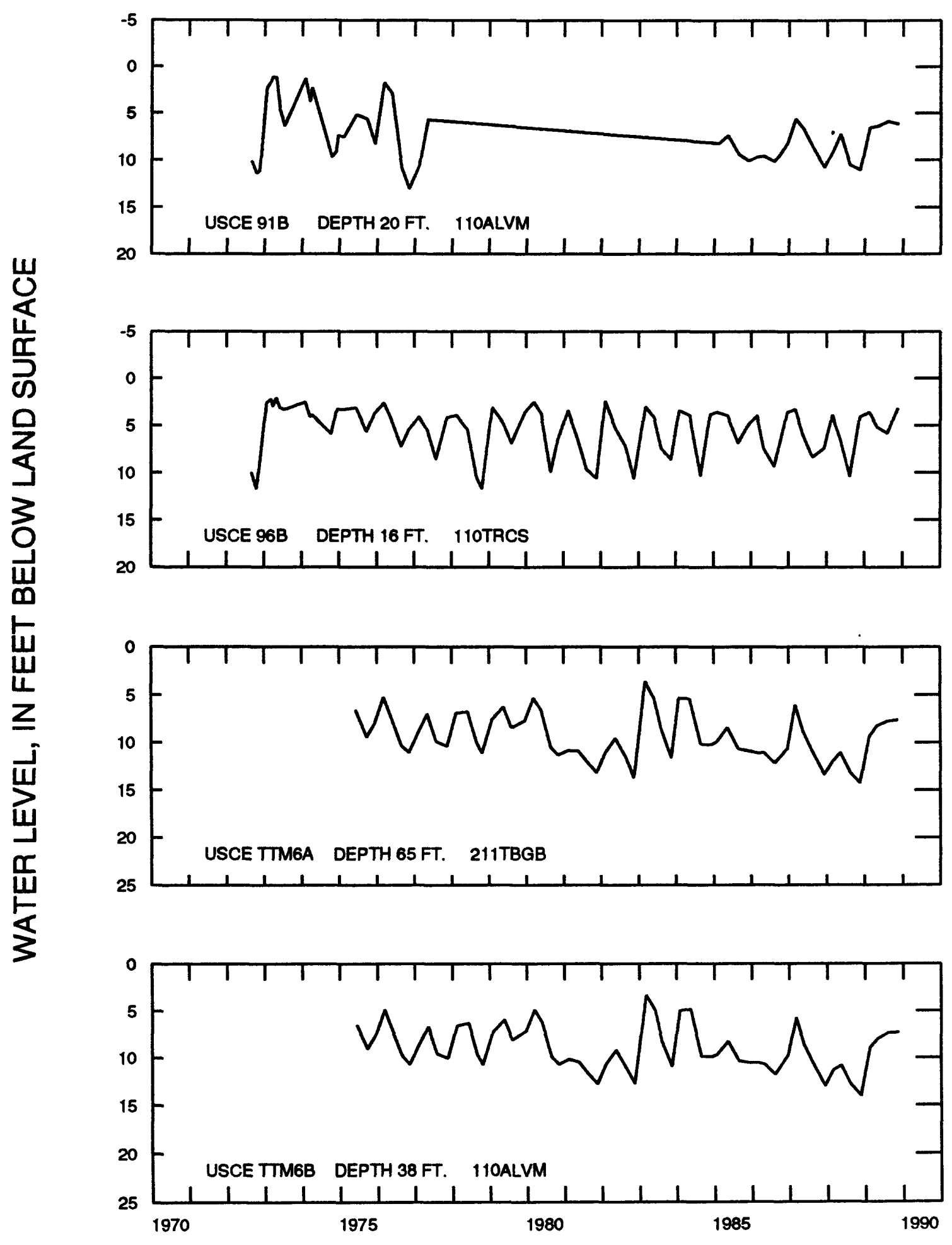

HYDROGRAPHS OF TENNESSEE-TOMBIGBEE OBSERVATION WELLS 


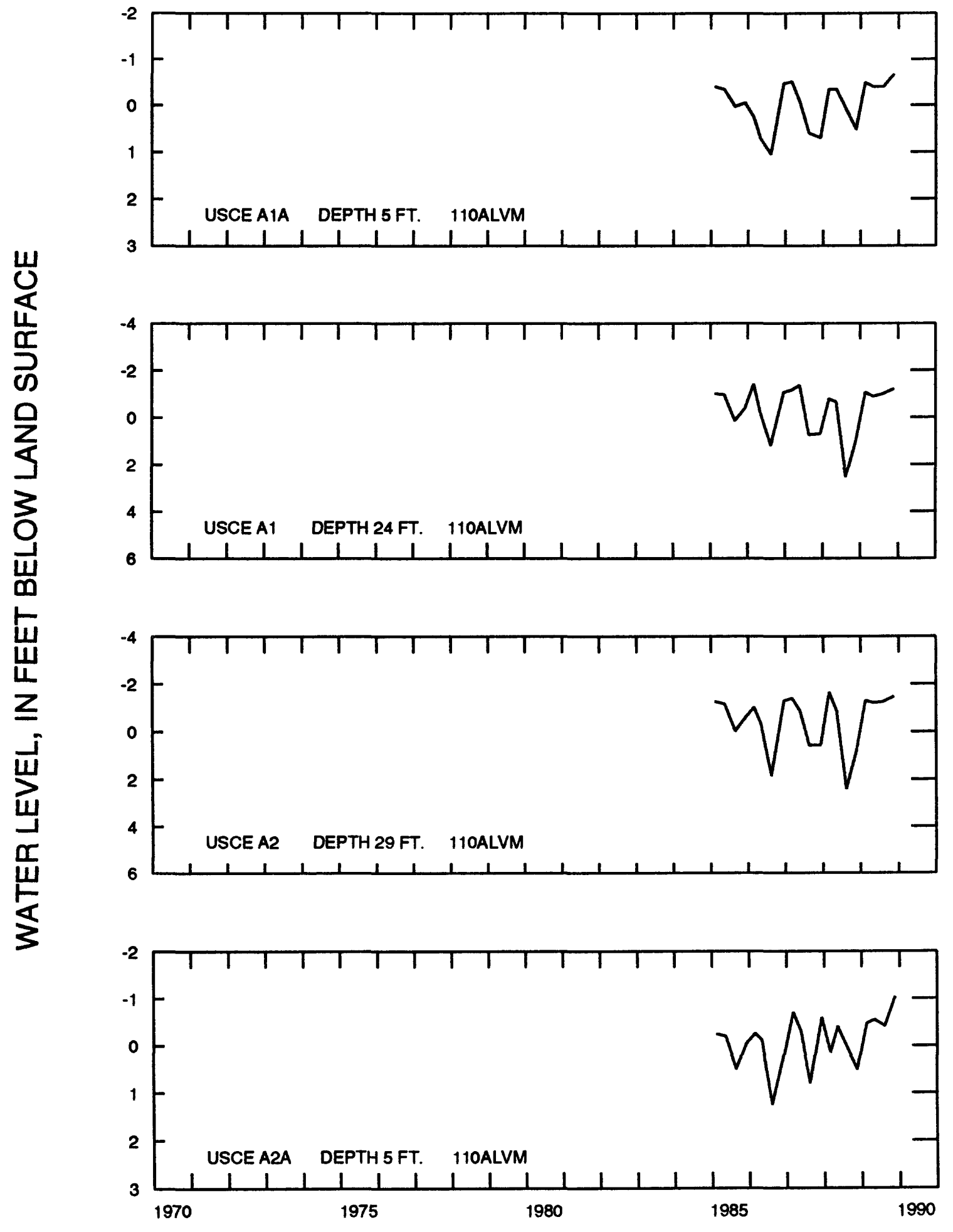

HYDROGRAPHS OF TENNESSEE-TOMBIGBEE OBSERVATION WELLS 


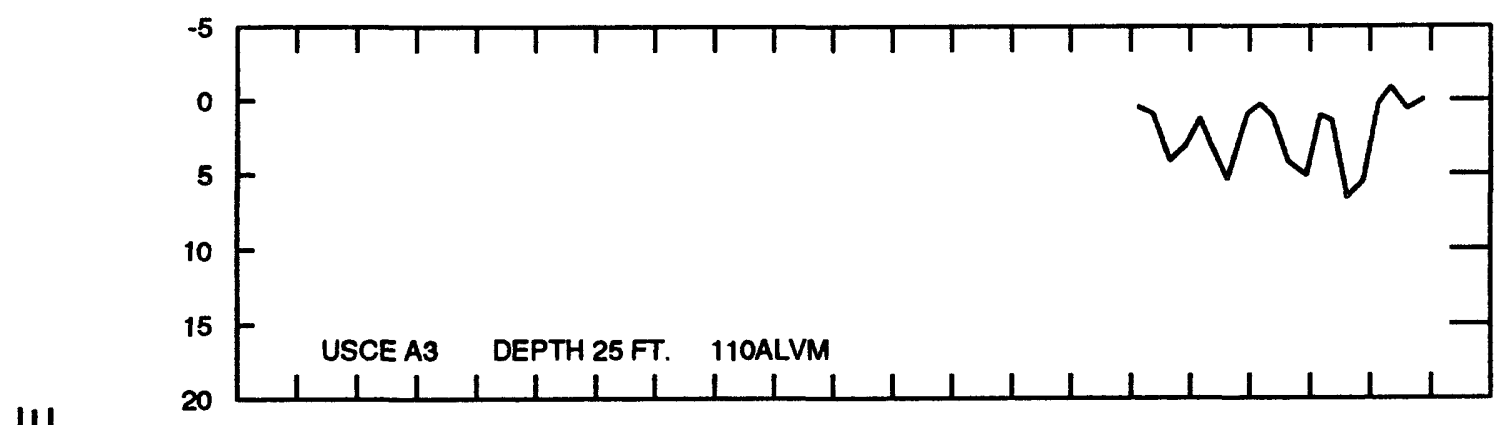

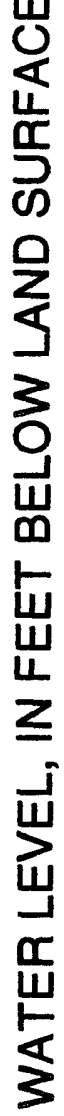
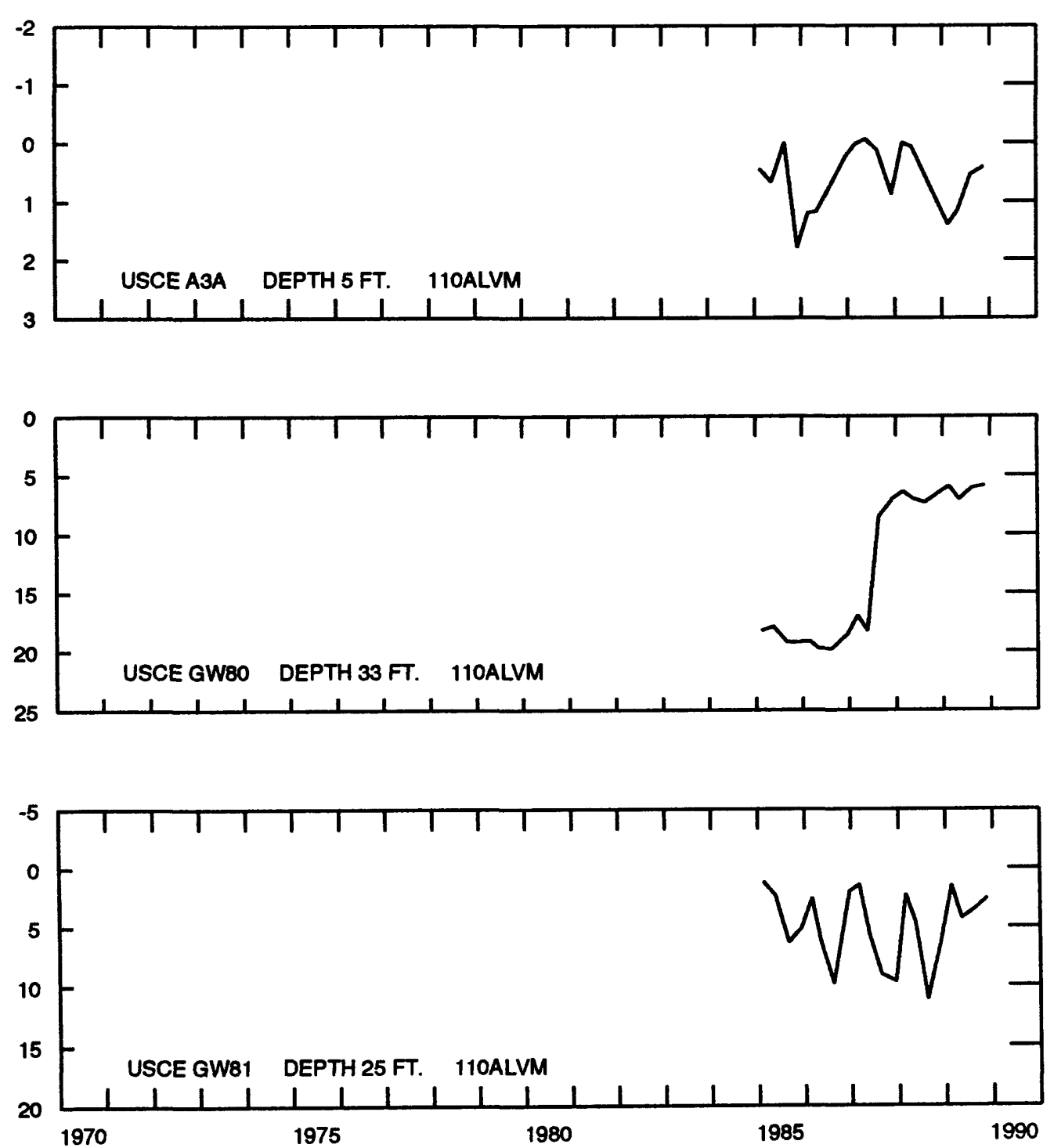

HYDROGRAPHS OF TENNESSEE-TOMBIGBEE OBSERVATION WELLS 


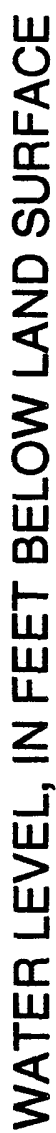
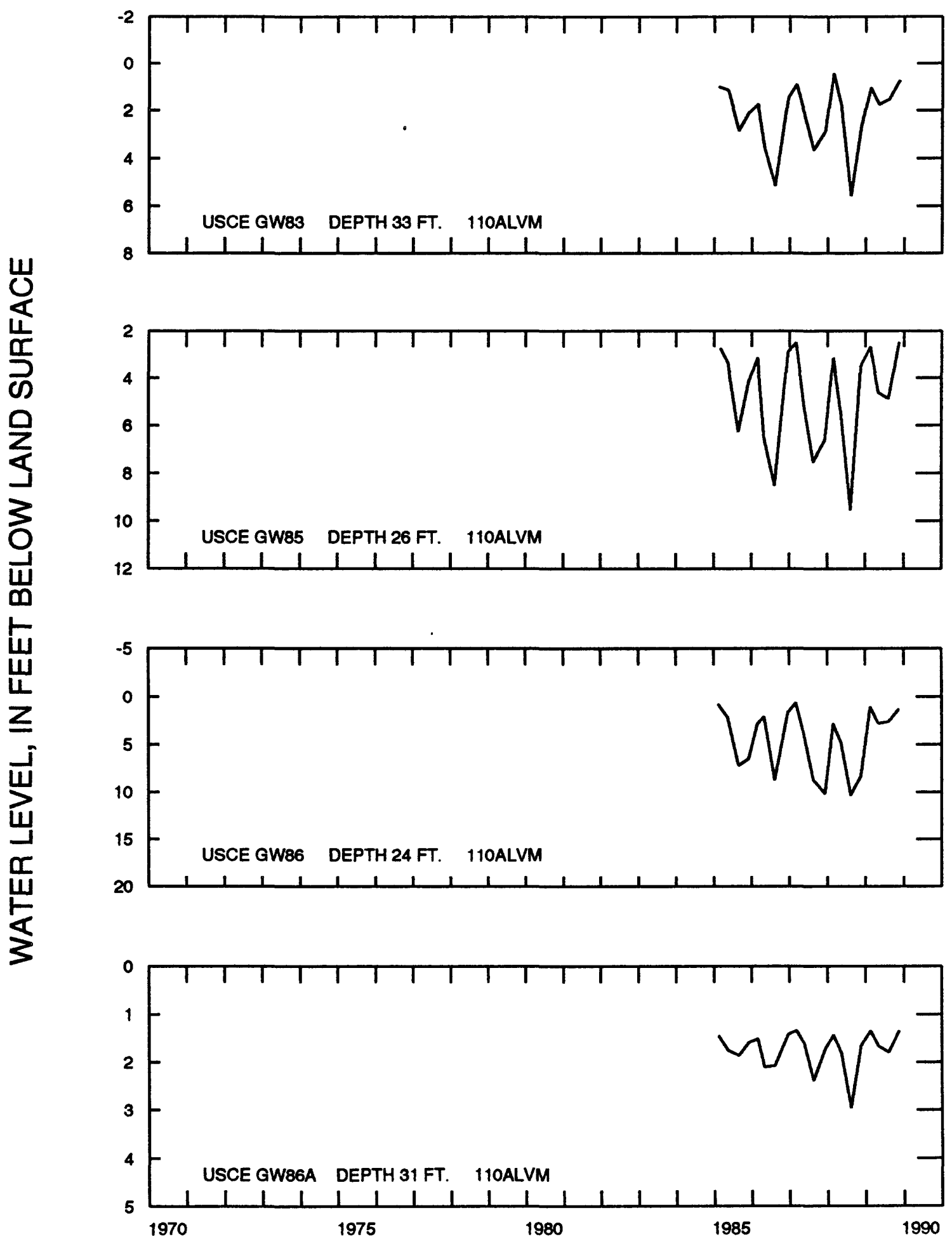

HYDROGRAPHS OF TENNESSEE-TOMBIGBEE OBSERVATION WELLS 


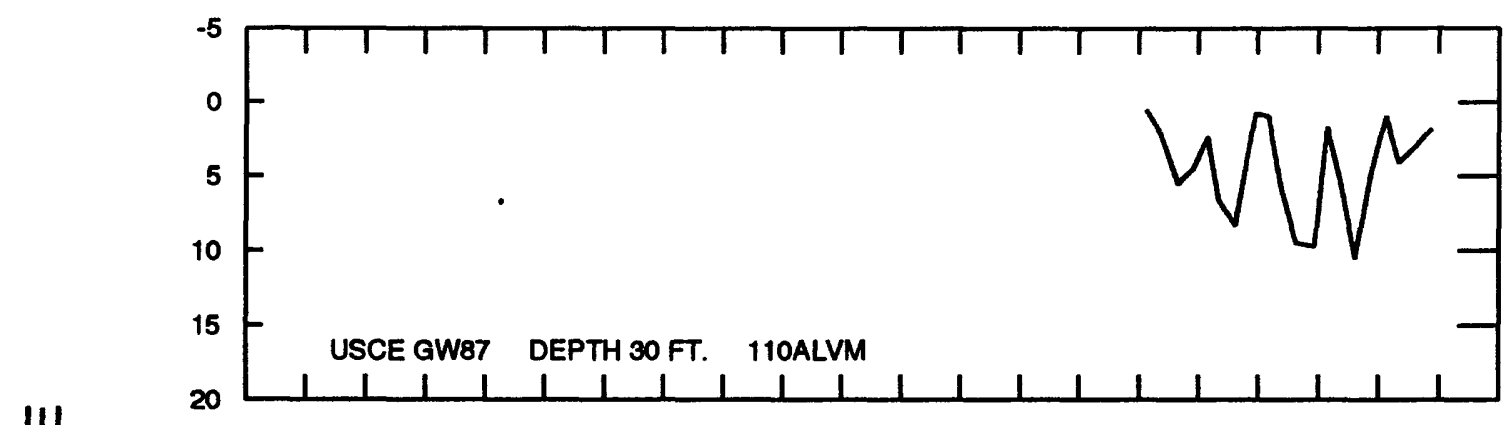

岁

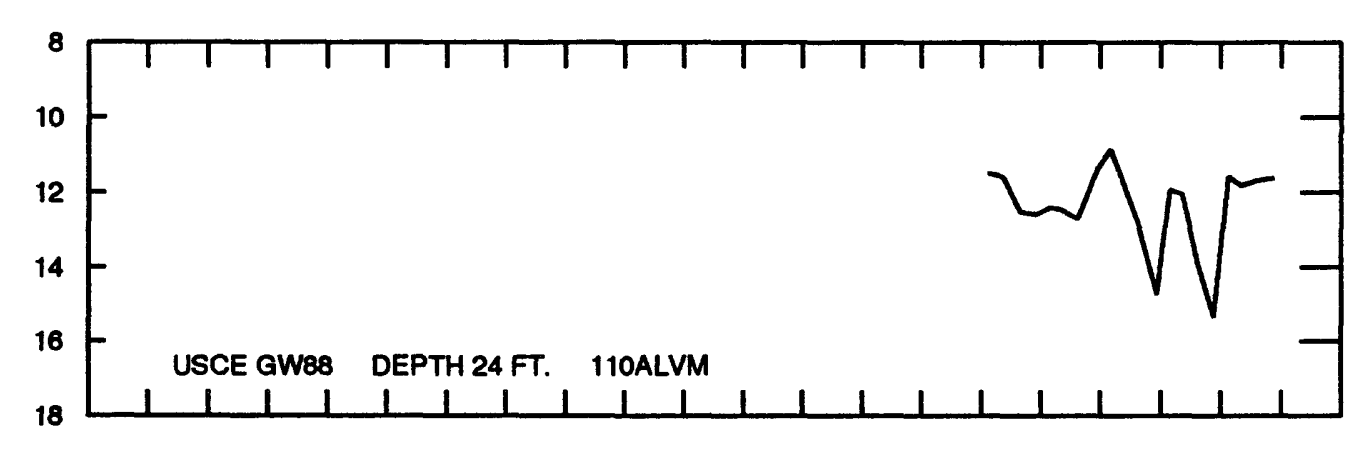

m

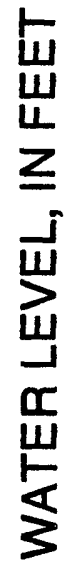

HYDROGRAPHS OF TENNESSEE-TOMBIGBEE OBSERVATION WELLS 


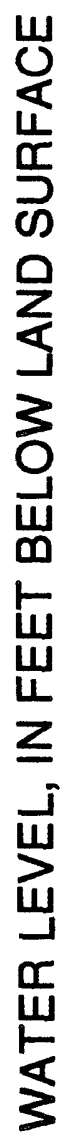
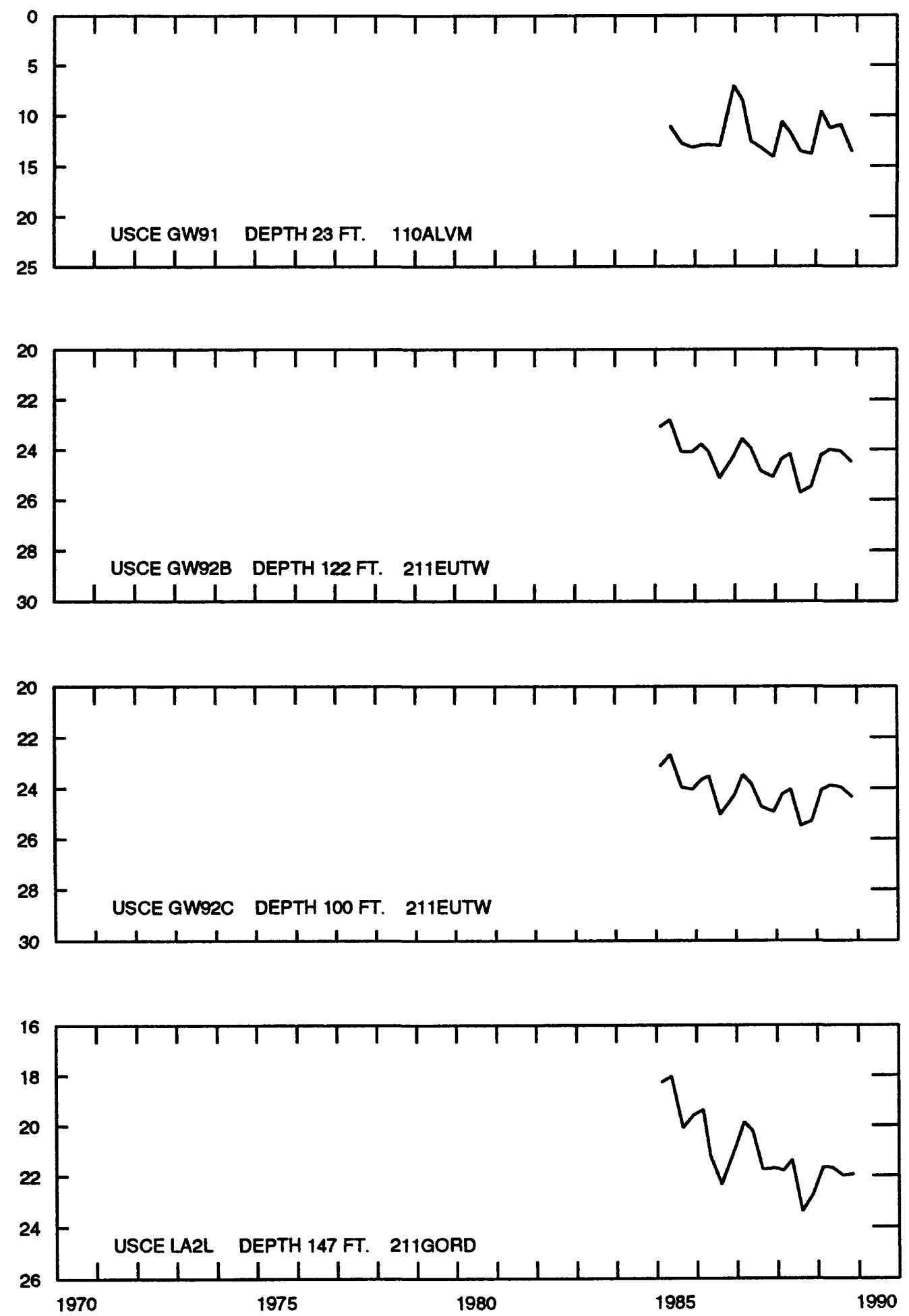

HYDROGRAPHS OF TENNESSEE-TOMBIGBEE OBSERVATION WELLS 


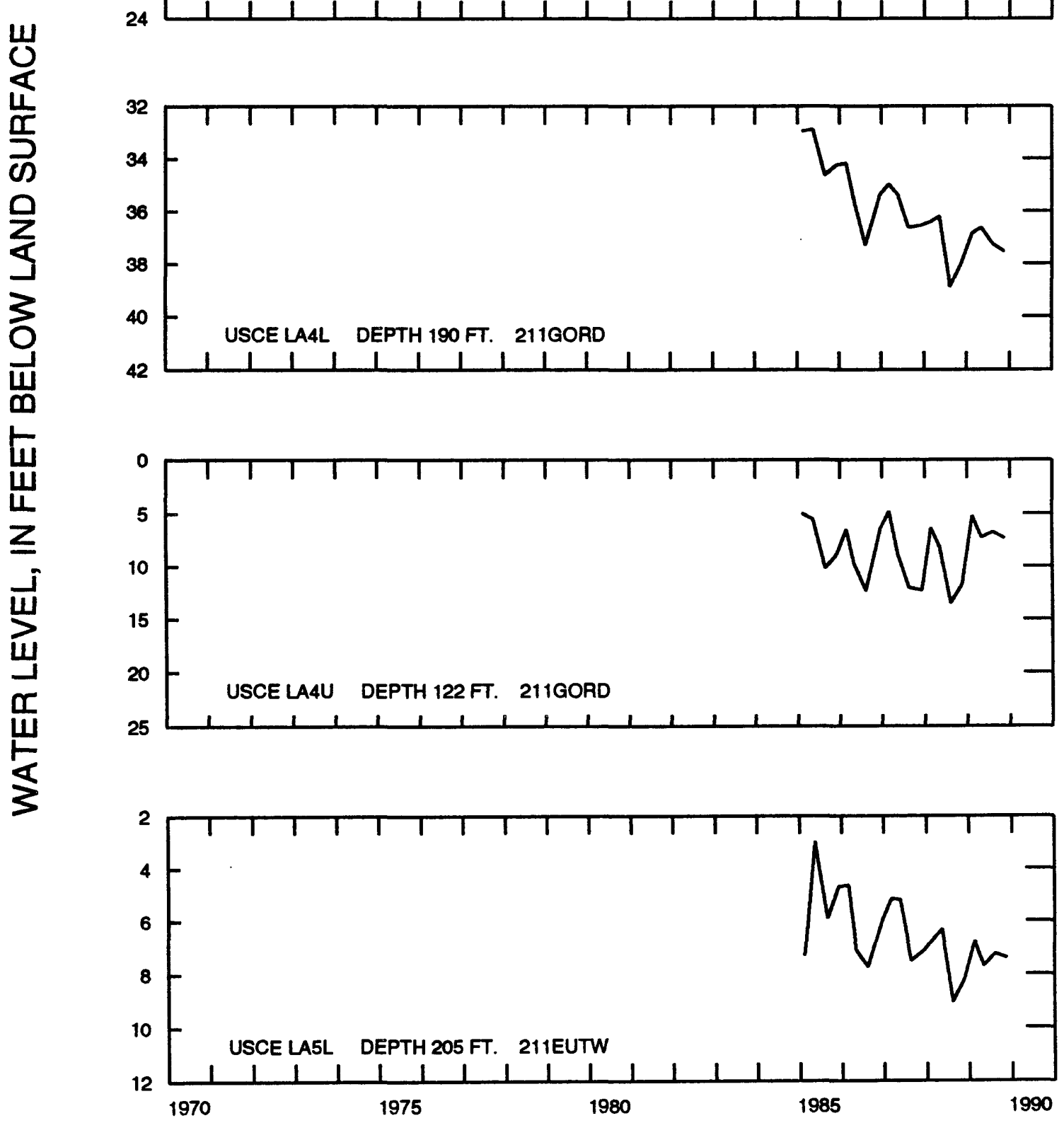

HYDROGRAPHS OF TENNESSEE-TOMBIGBEE OBSERVATION WELLS 


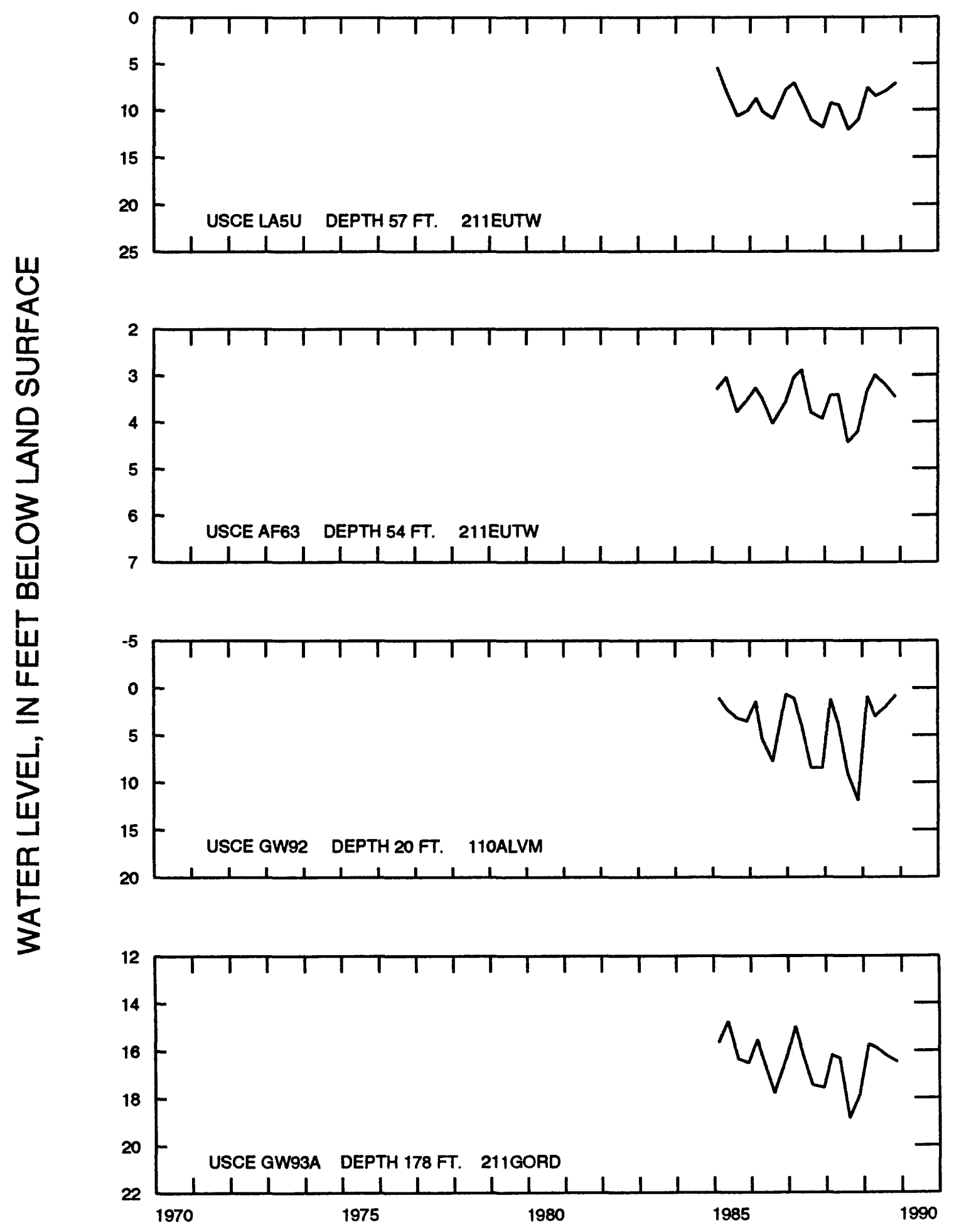

HYDROGRAPHS OF TENNESSEE-TOMBIGBEE OBSERVATION WELLS 


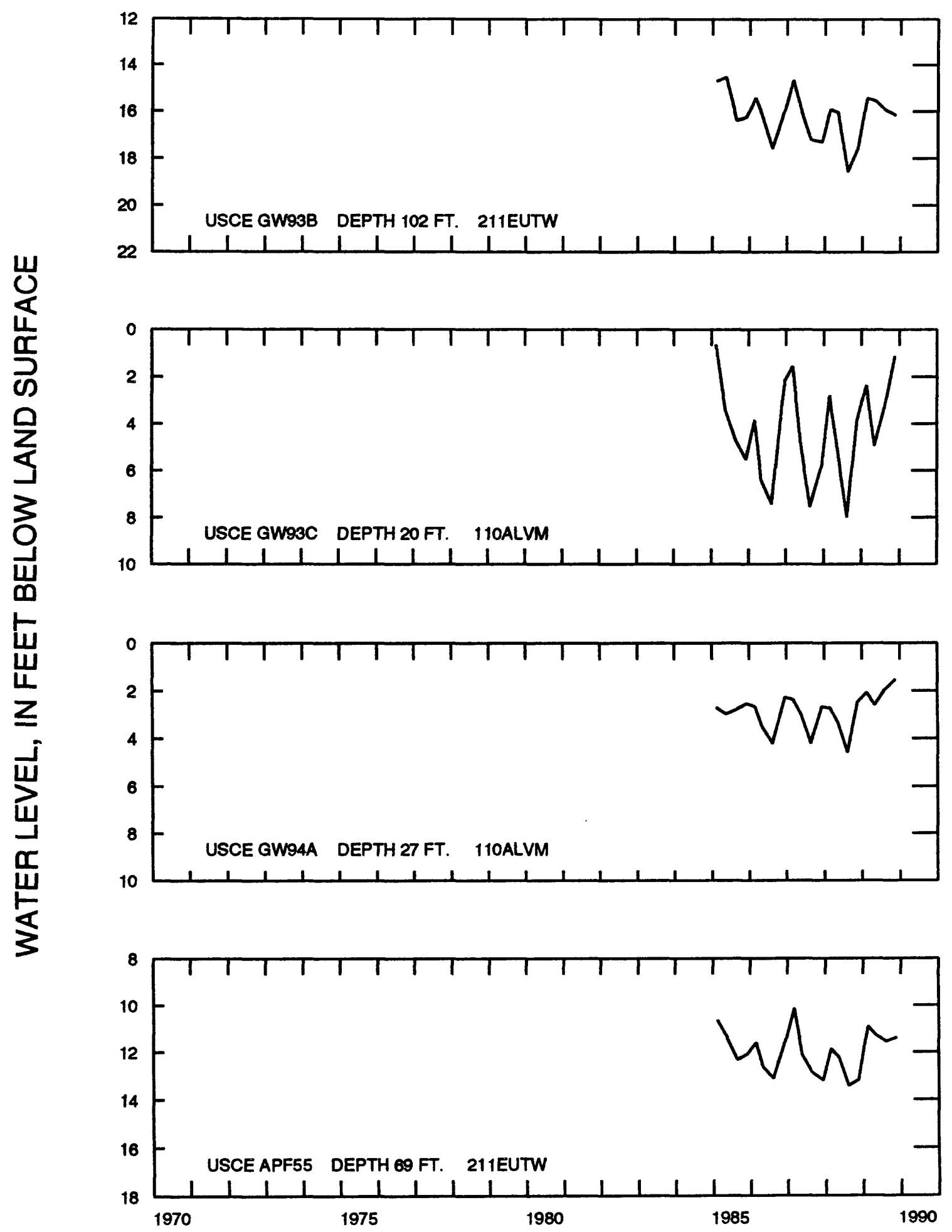

HYDROGRAPHS OF TENNESSEE-TOMBIGBEE OBSERVATION WELLS 


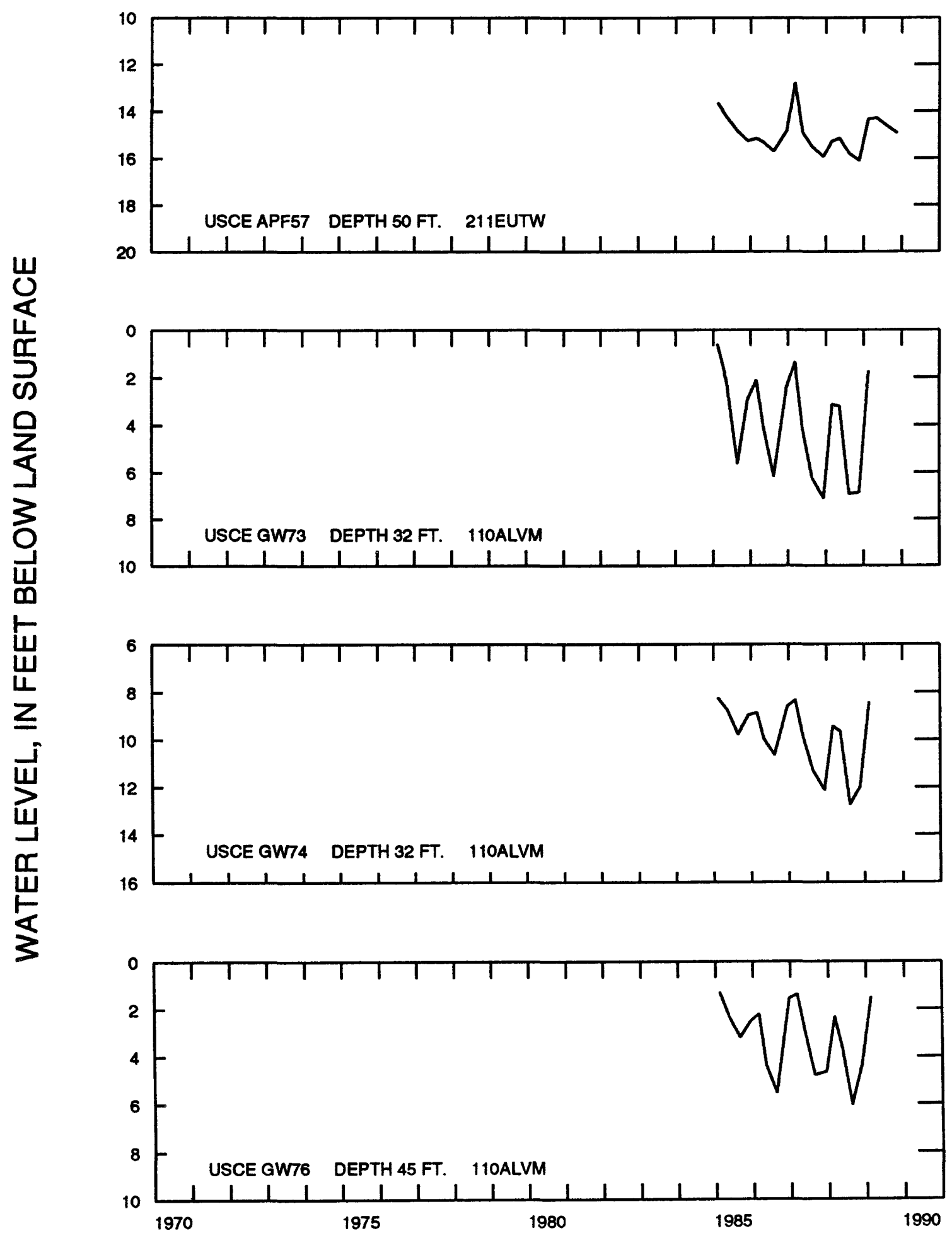

HYDROGRAPHS OF TENNESSEE-TOMBIGBEE OBSERVATION WELLS 


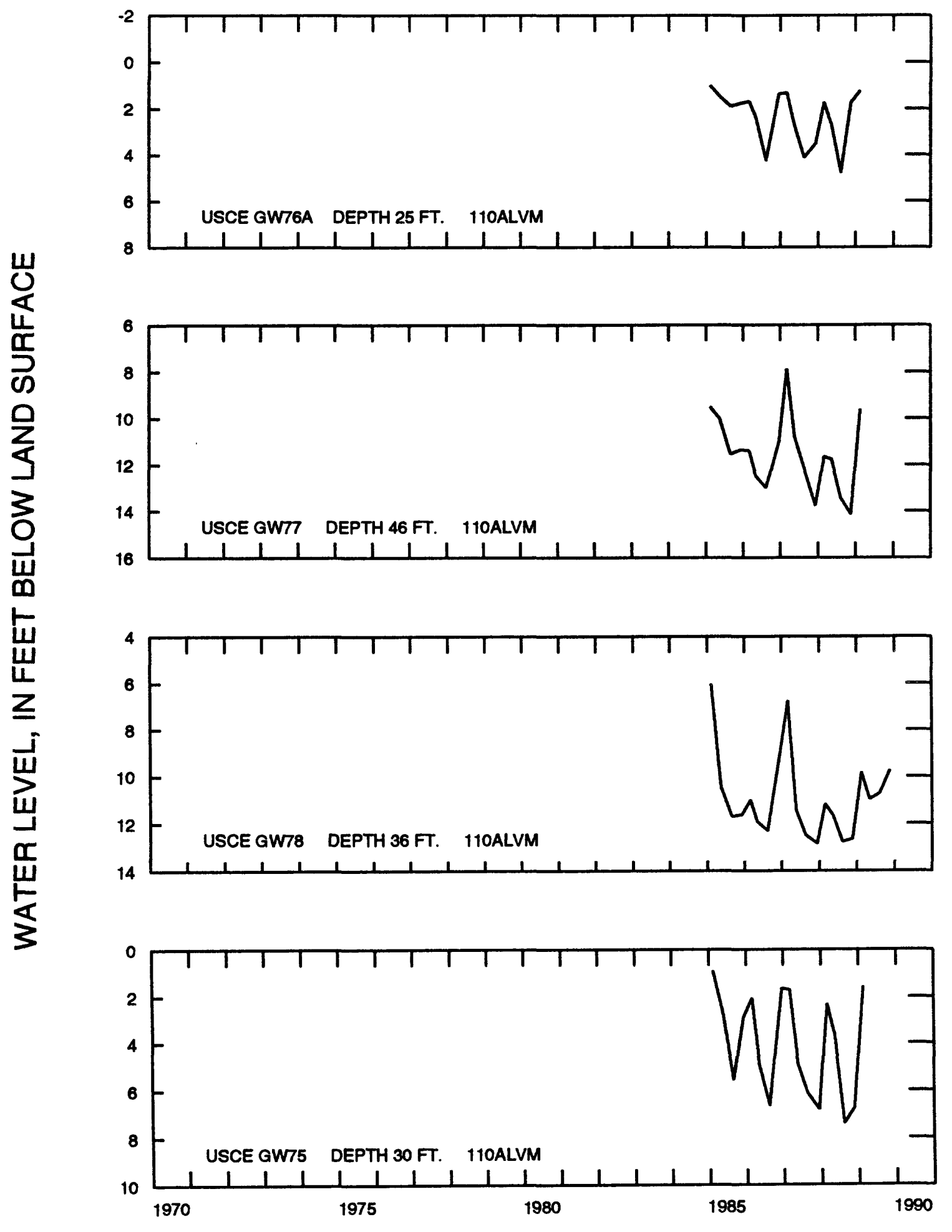

HYDROGRAPHS OF TENNESSEE-TOMBIGBEE OBSERVATION WELLS 


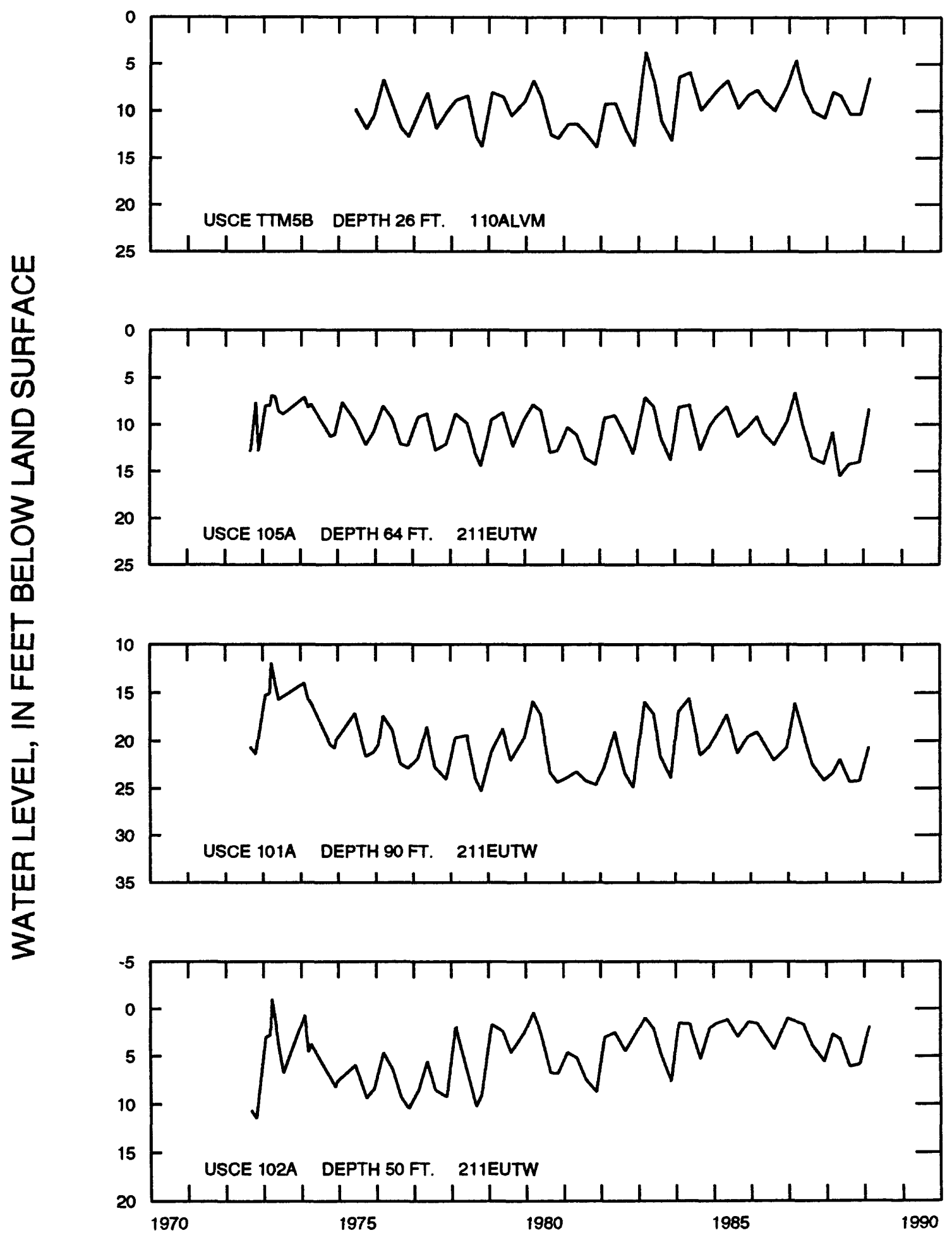

HYDROGRAPHS OF TENNESSEE-TOMBIGBEE OBSERVATION WELLS 


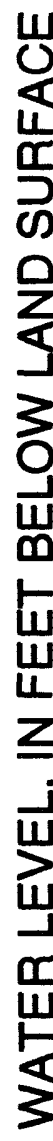

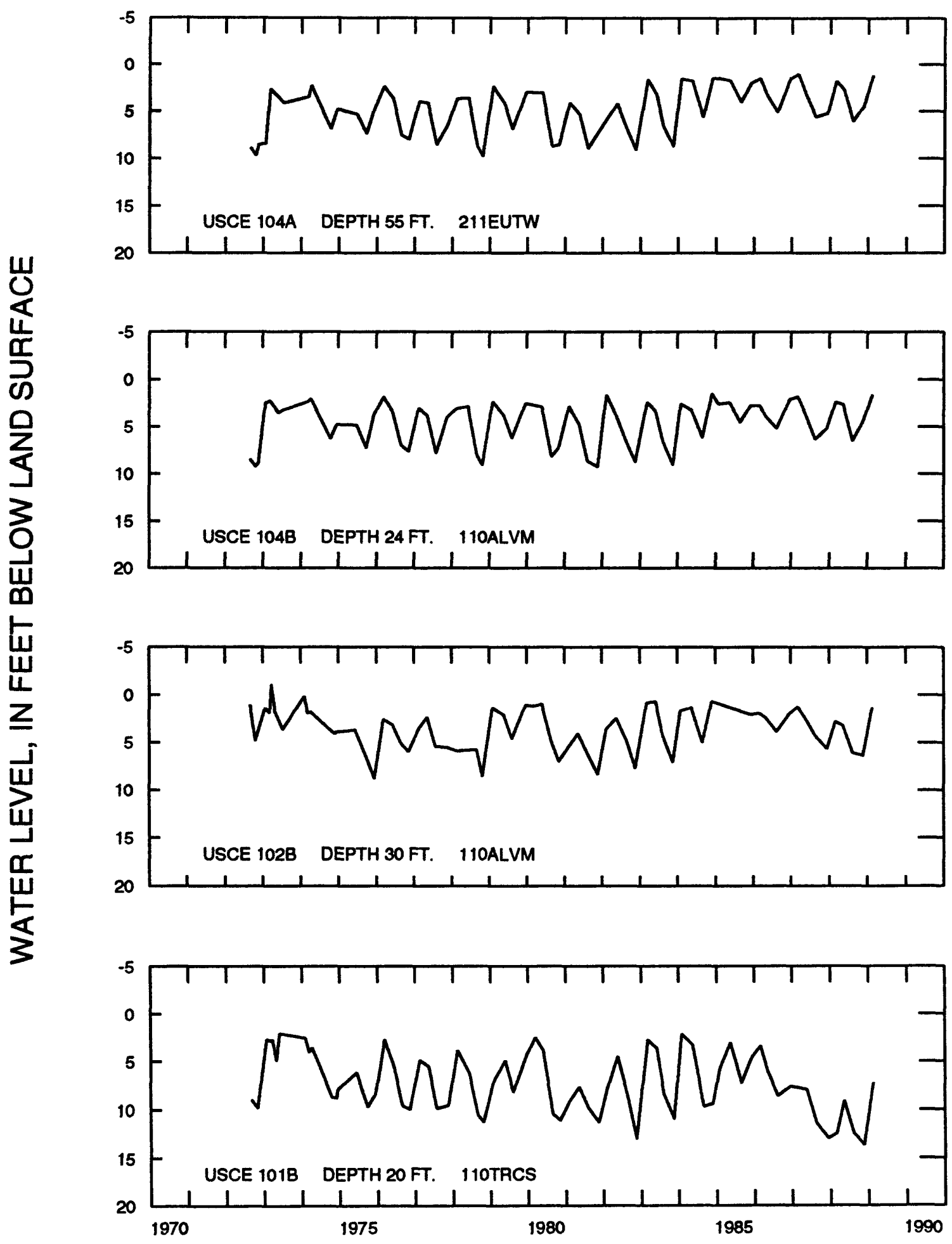

HYDROGRAPHS OF TENNESSEE-TOMBIGBEE OBSERVATION WELLS 


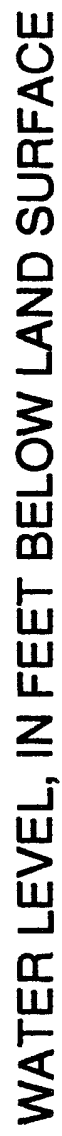
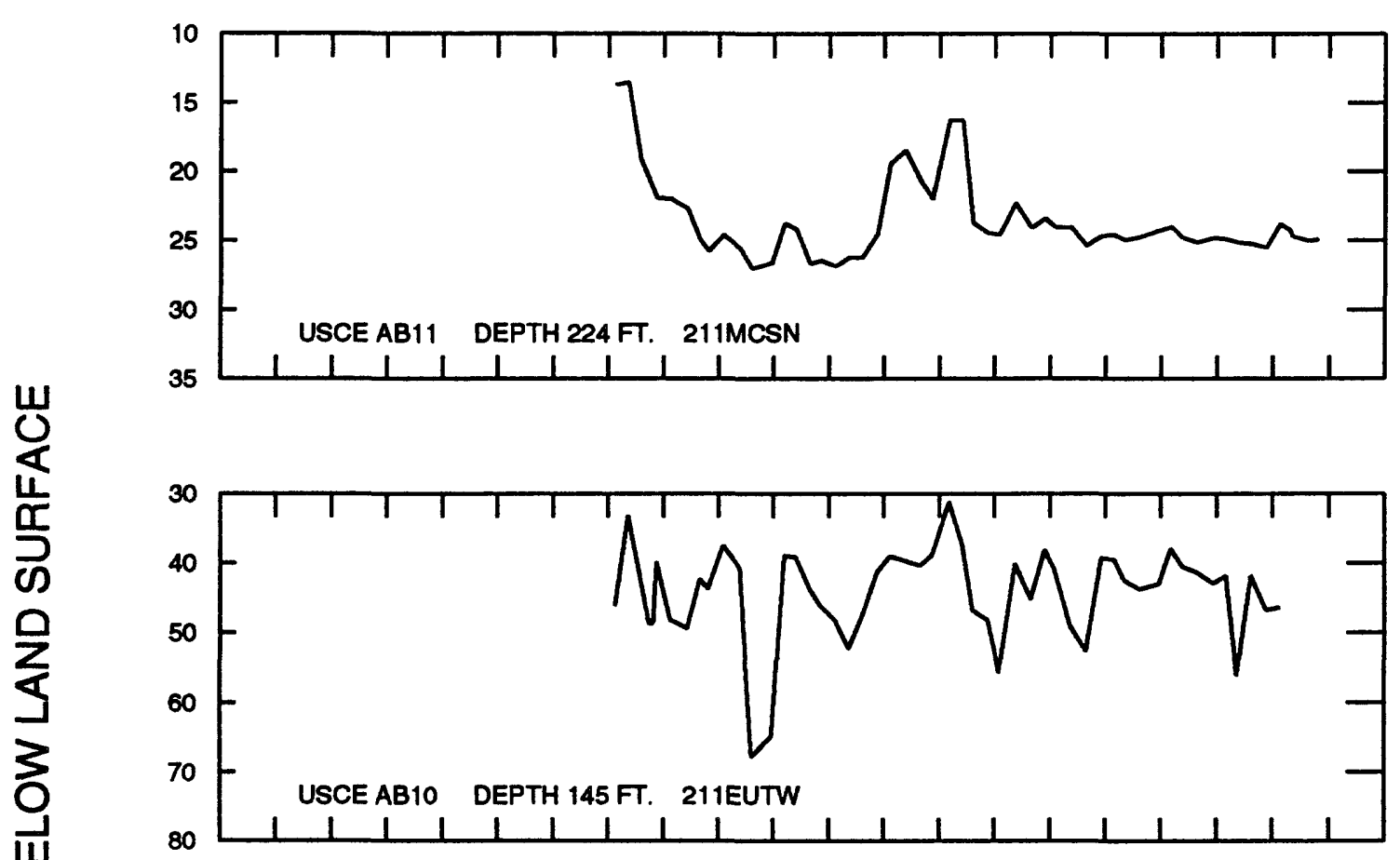

HYDROGRAPHS OF TENNESSEE-TOMBIGBEE OBSERVATION WELLS 


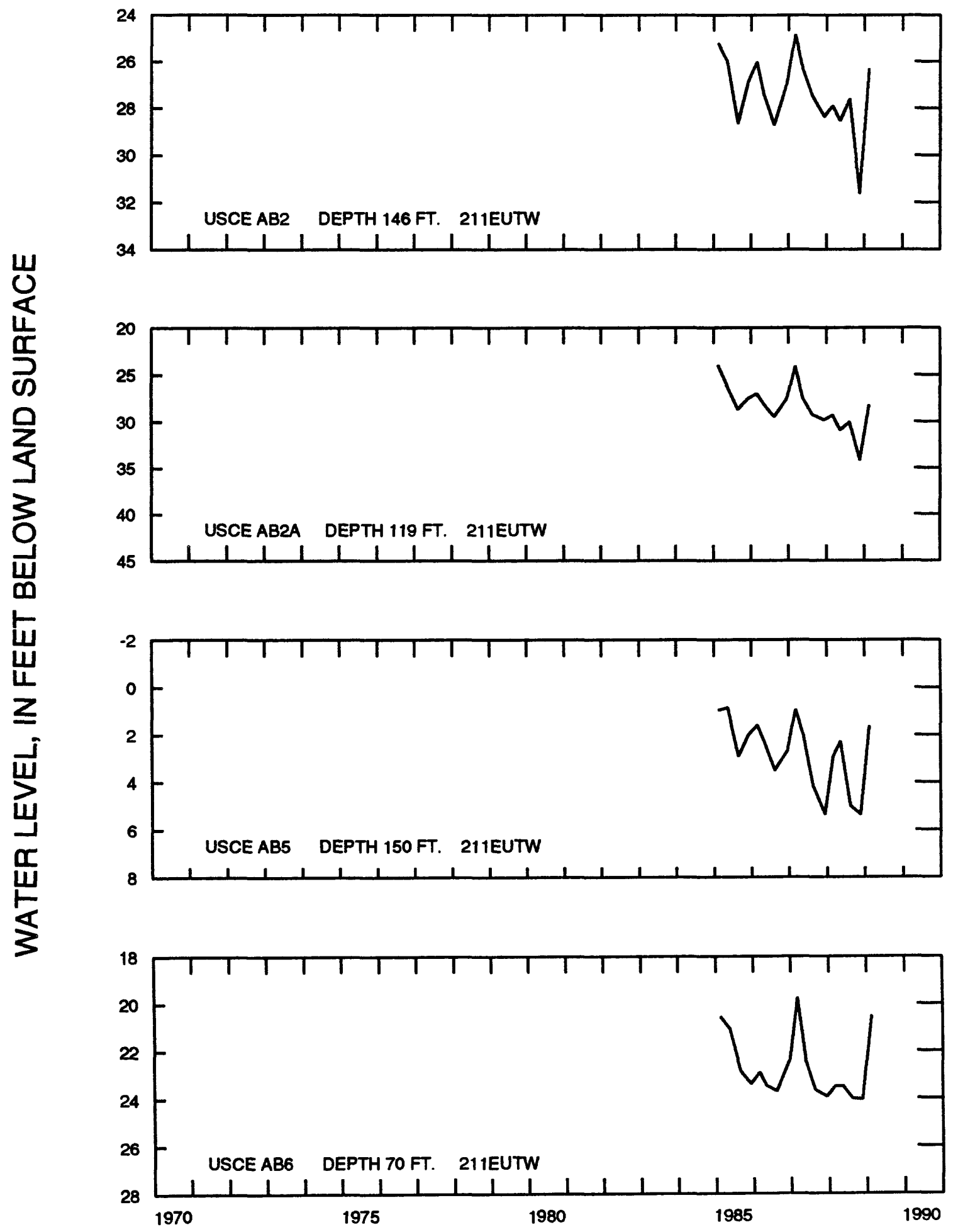

HYDROGRAPHS OF TENNESSEE-TOMBIGBEE OBSERVATION WELLS 


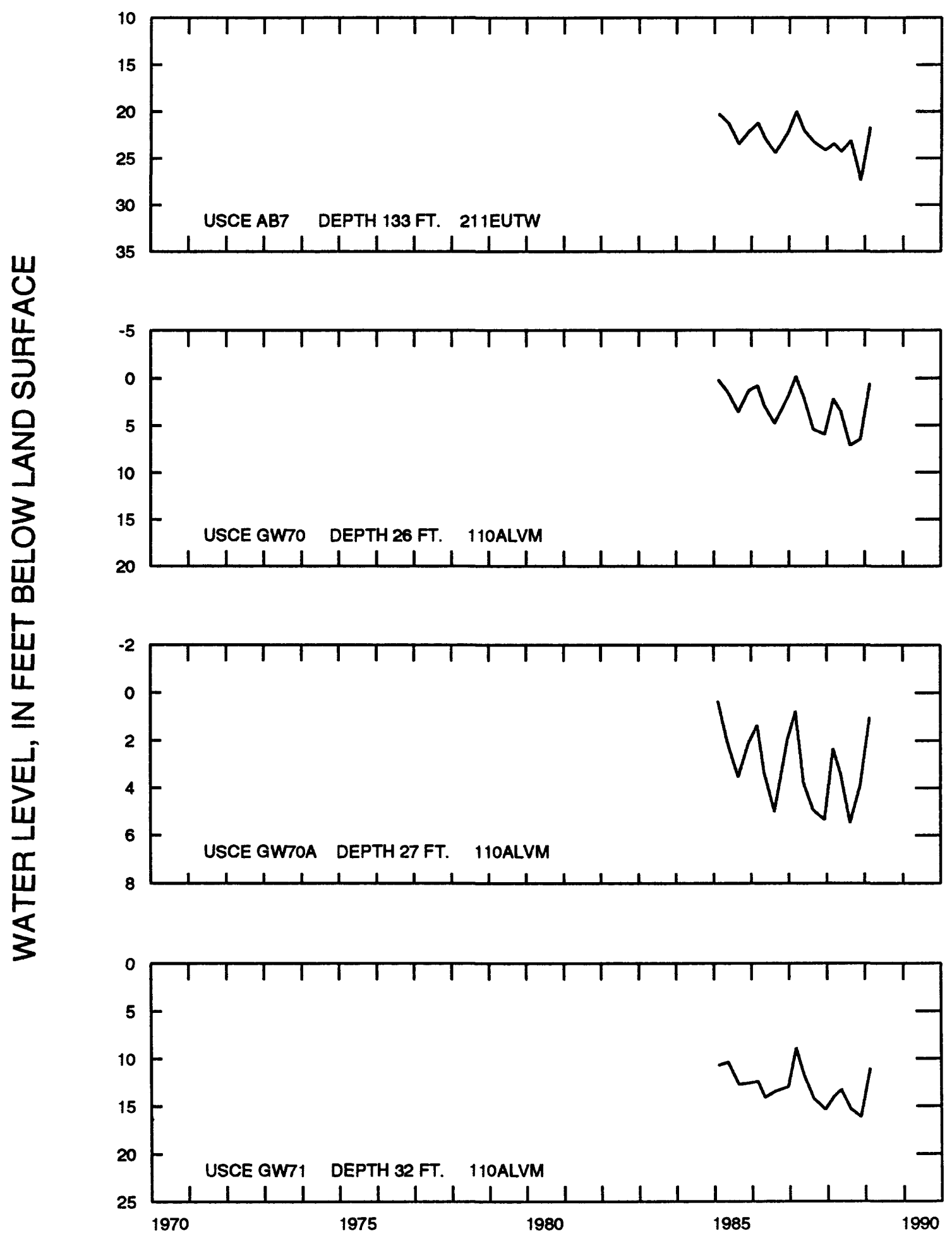

HYDROGRAPHS OF TENNESSEE-TOMBIGBEE OBSERVATION WELLS 


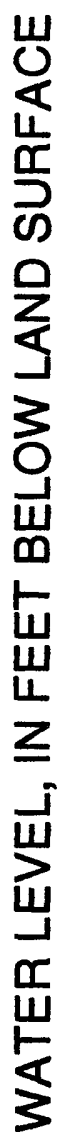
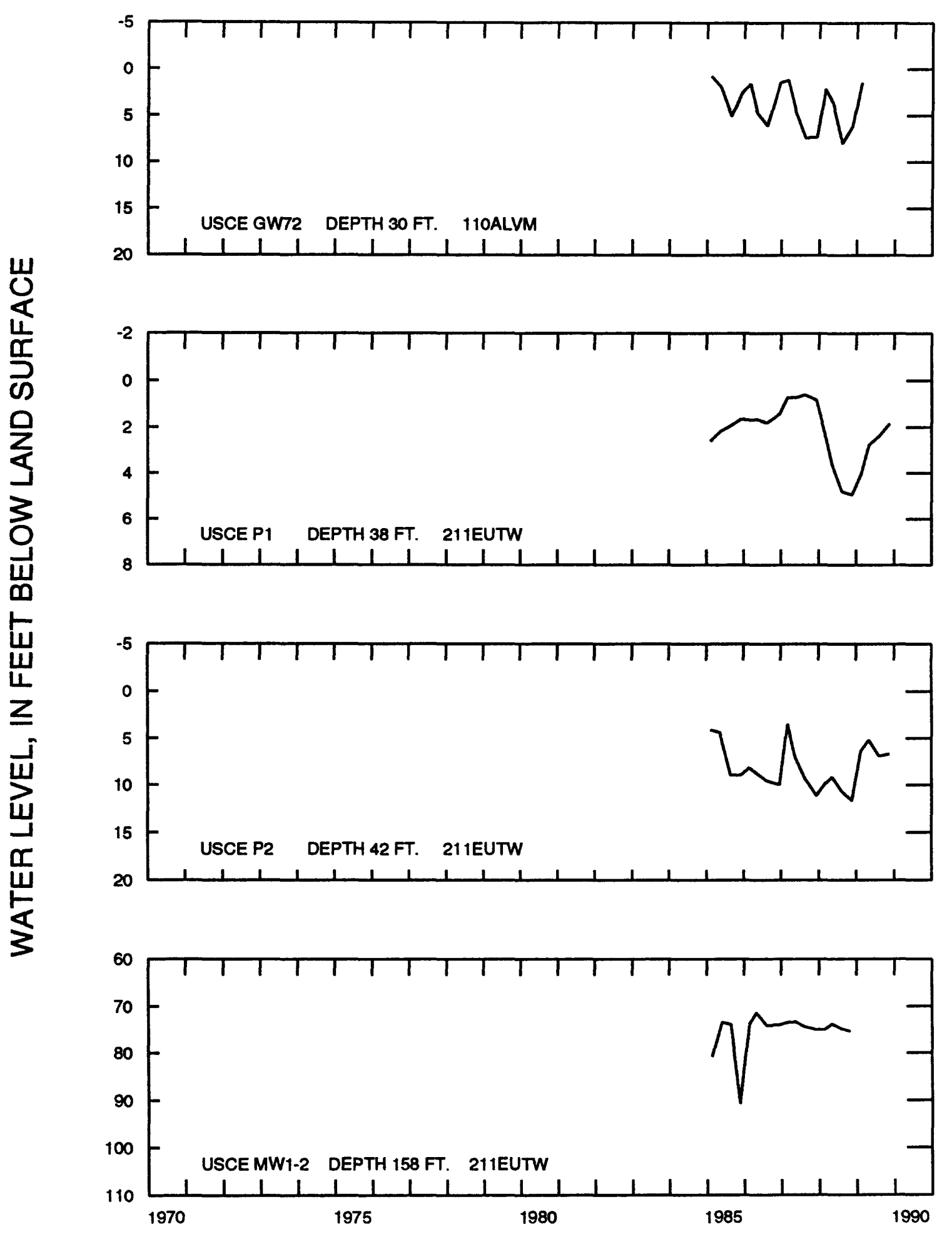

HYDROGRAPHS OF TENNESSEE-TOMBIGBEE OBSERVATION WELLS 


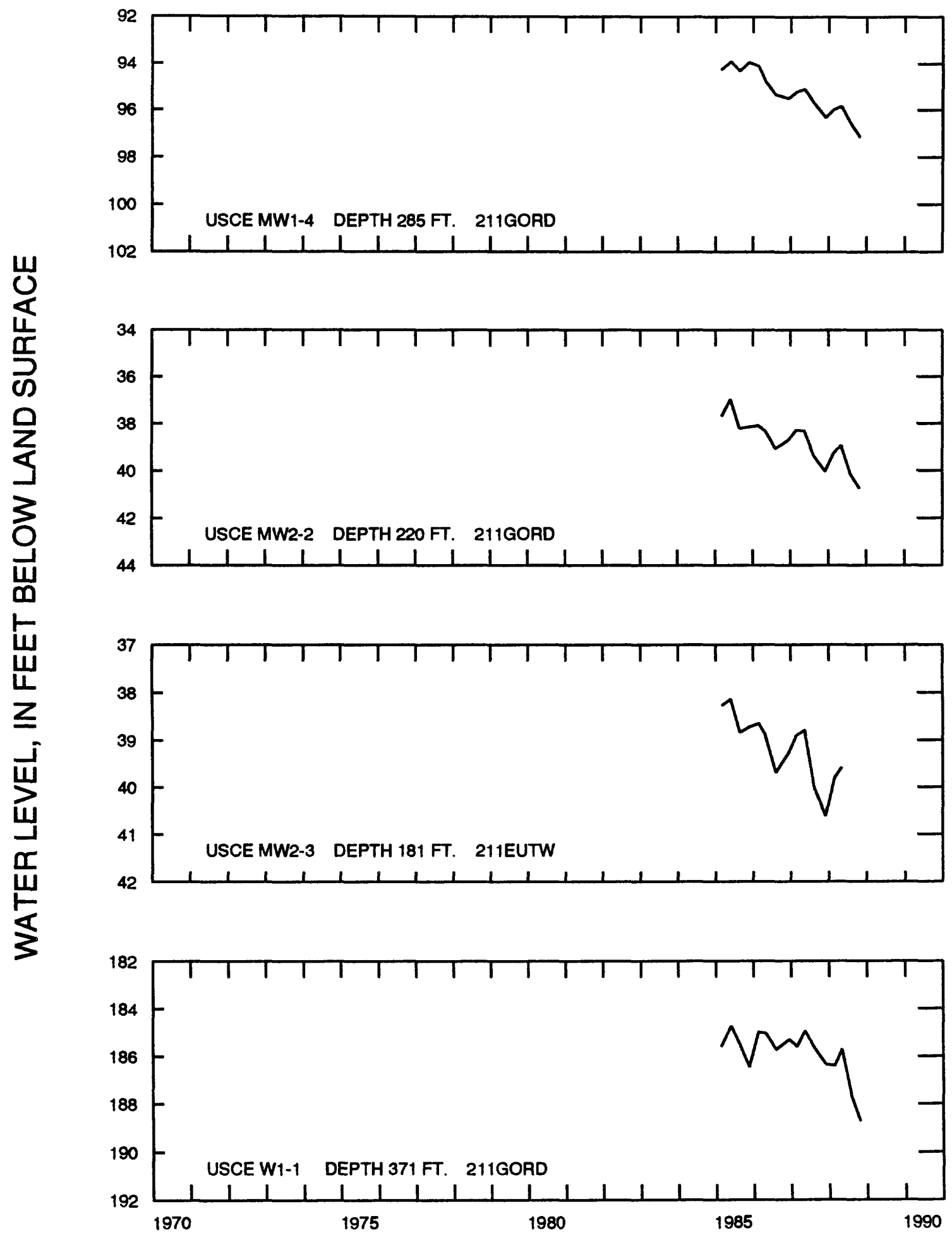

HYDROGRAPHS OF TENNESSEE-TOMBIGBEE OBSERVATION WELLS 


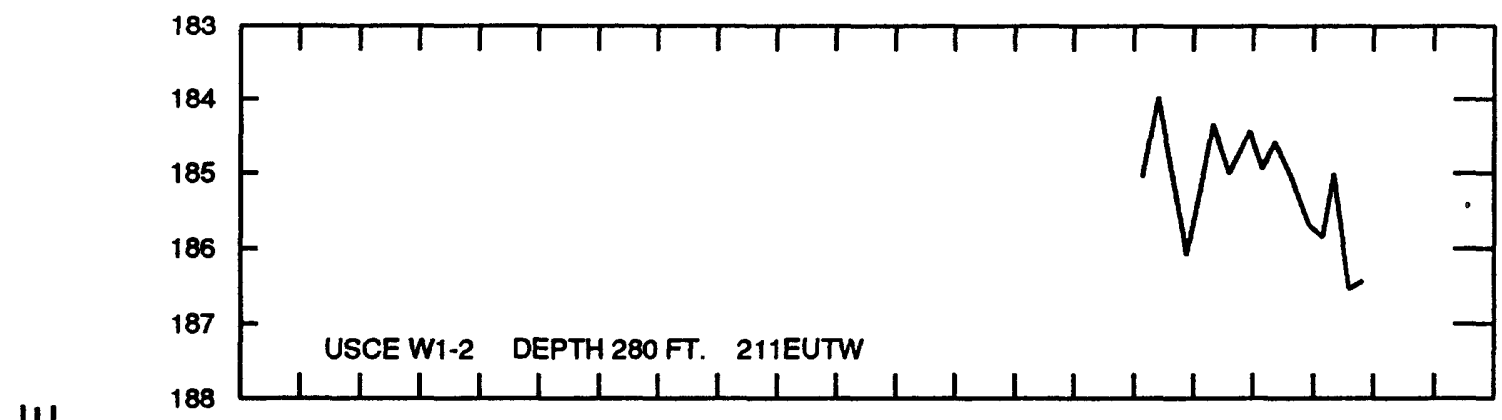

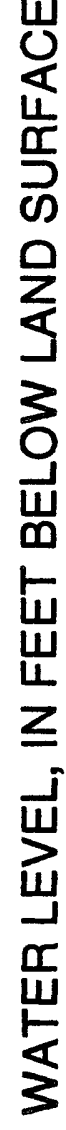
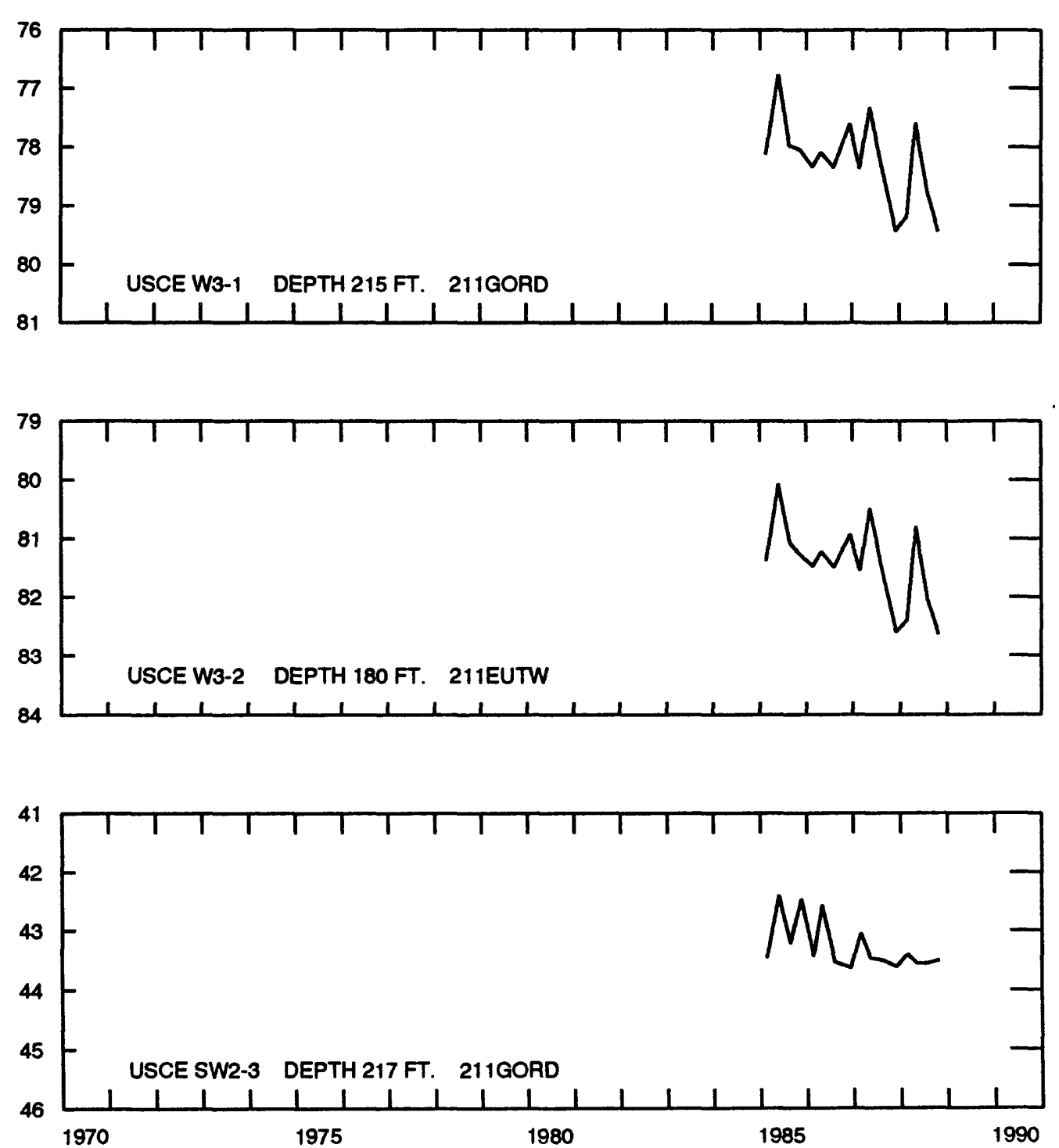

HYDROGRAPHS OF TENNESSEE-TOMBIGBEE OBSERVATION WELLS 


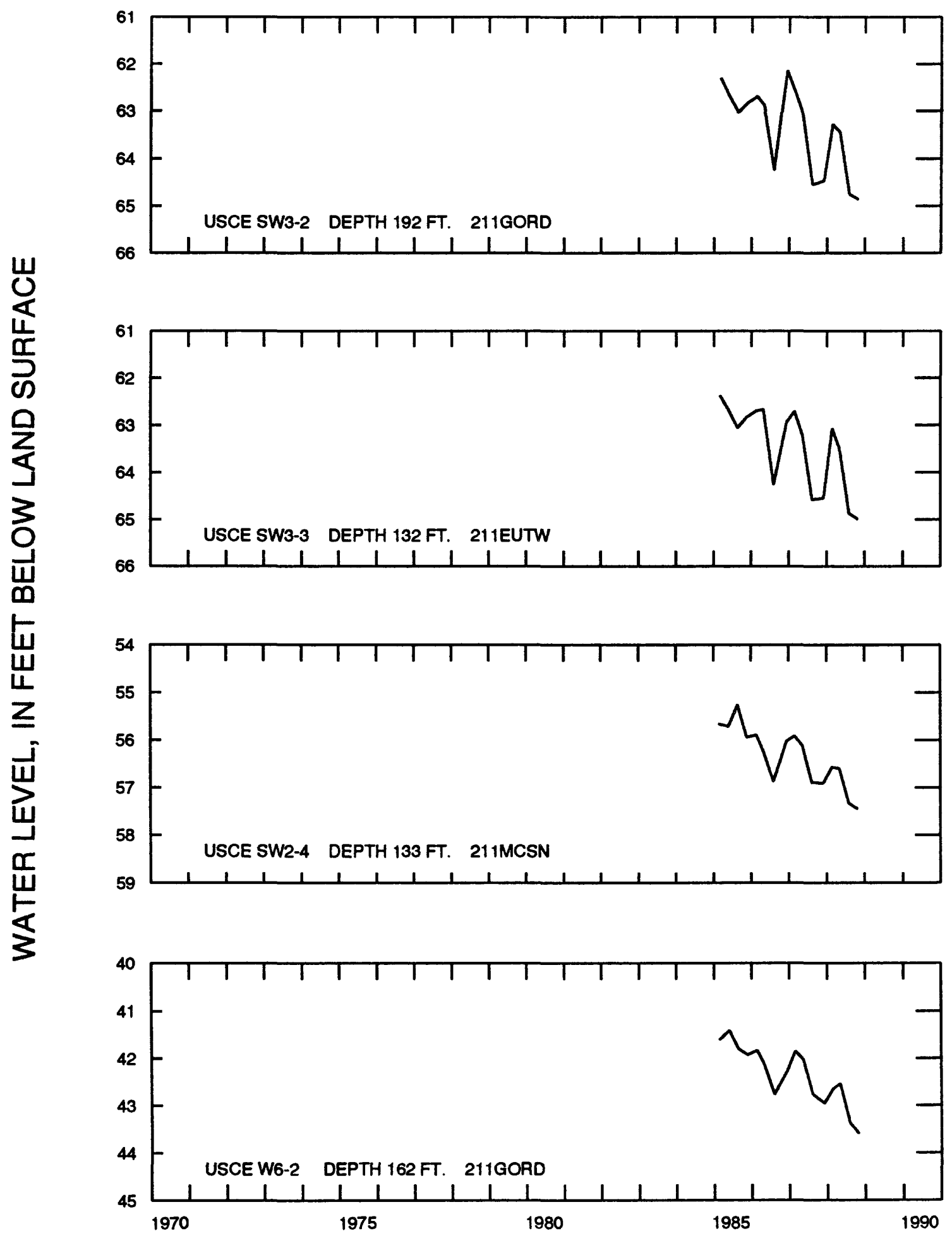

HYDROGRAPHS OF TENNESSEE-TOMBIGBEE OBSERVATION WELLS 


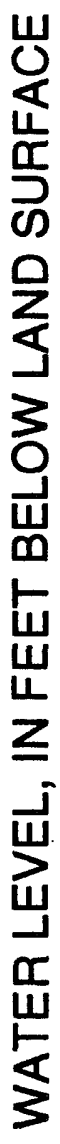
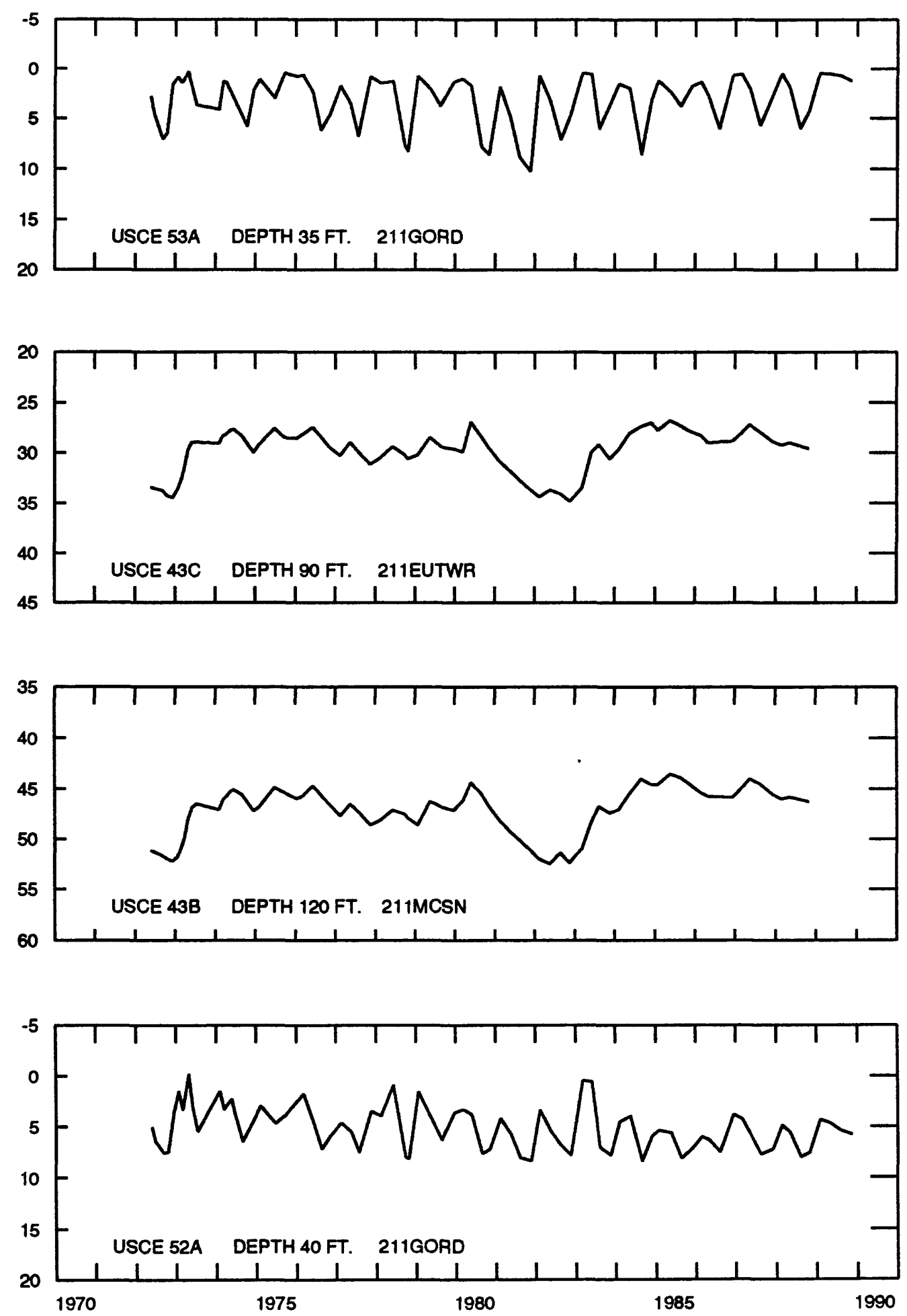

HYDROGRAPHS OF TENNESSEE-TOMBIGBEE OBSERVATION WELLS 


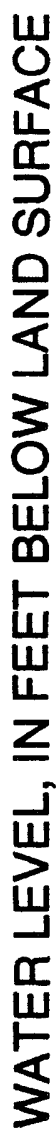
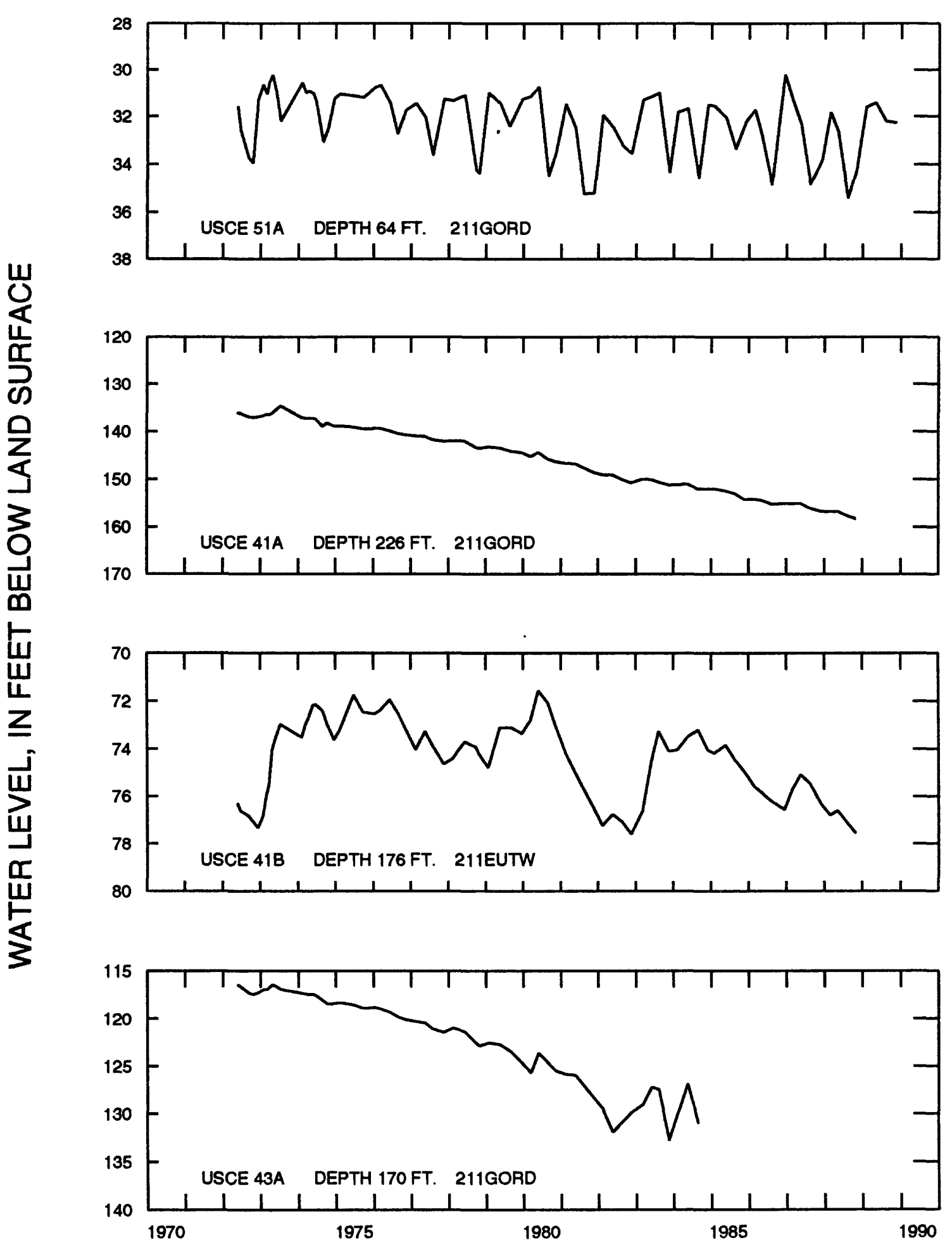

HYDROGRAPHS OF TENNESSEE-TOMBIGBEE OBSERVATION WELLS 


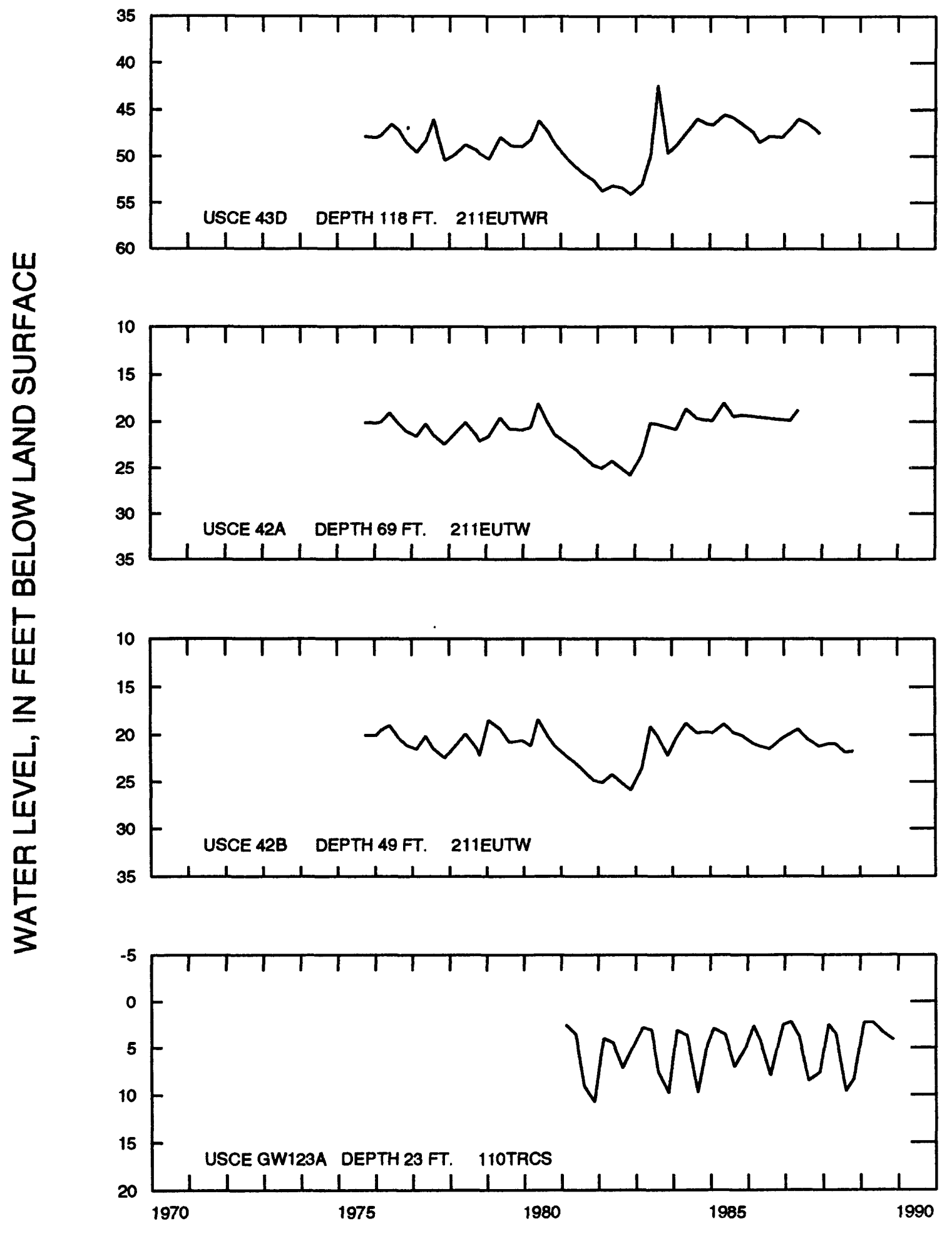

HYDROGRAPHS OF TENNESSEE-TOMBIGBEE OBSERVATION WELLS 


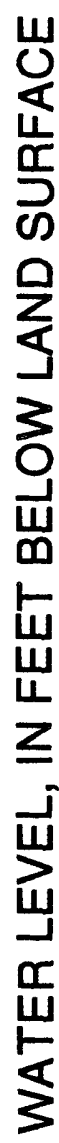

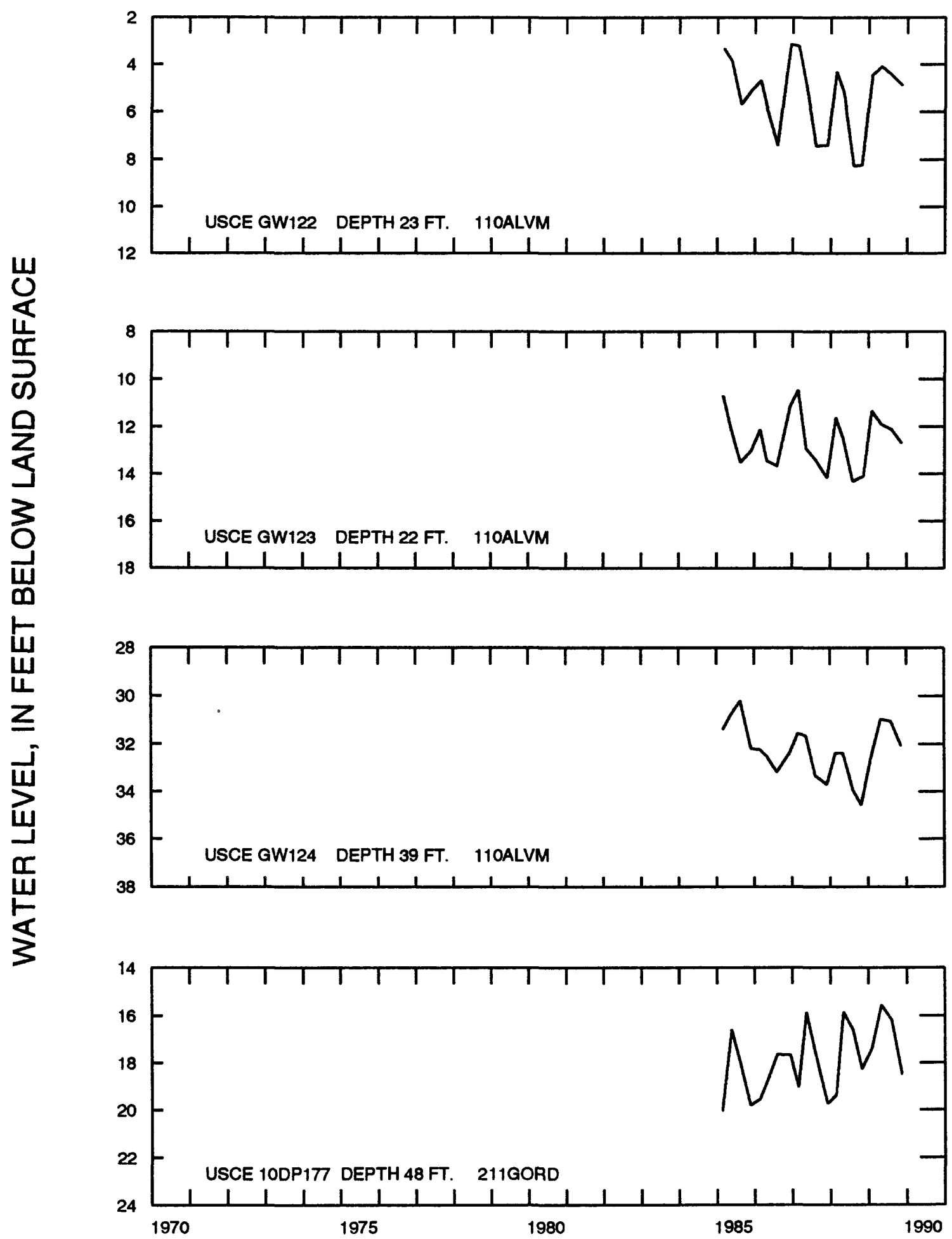

HYDROGRAPHS OF TENNESSEE-TOMBIGBEE OBSERVATION WELLS 


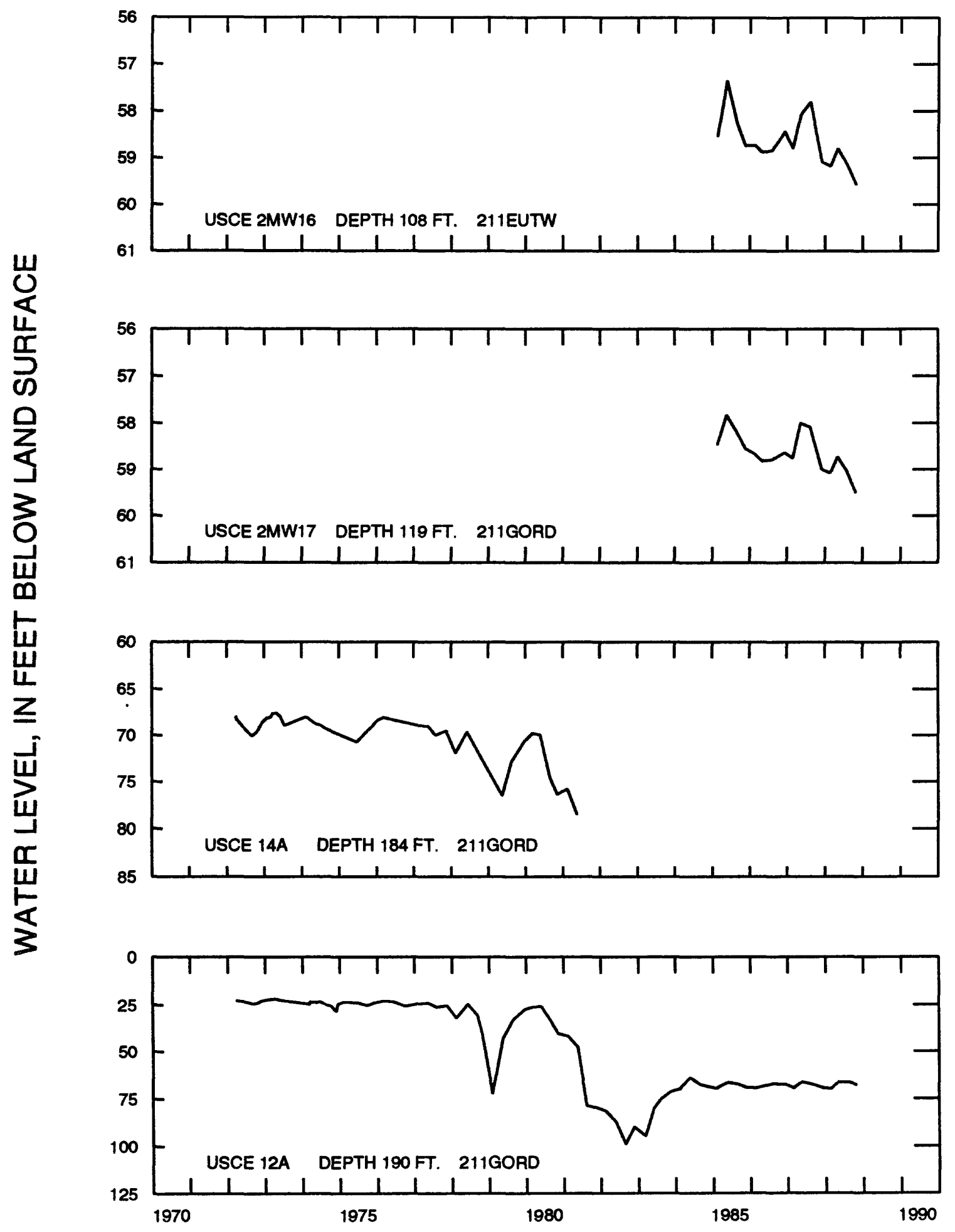

HYDROGRAPHS OF TENNESSEE-TOMBIGBEE OBSERVATION WELLS 


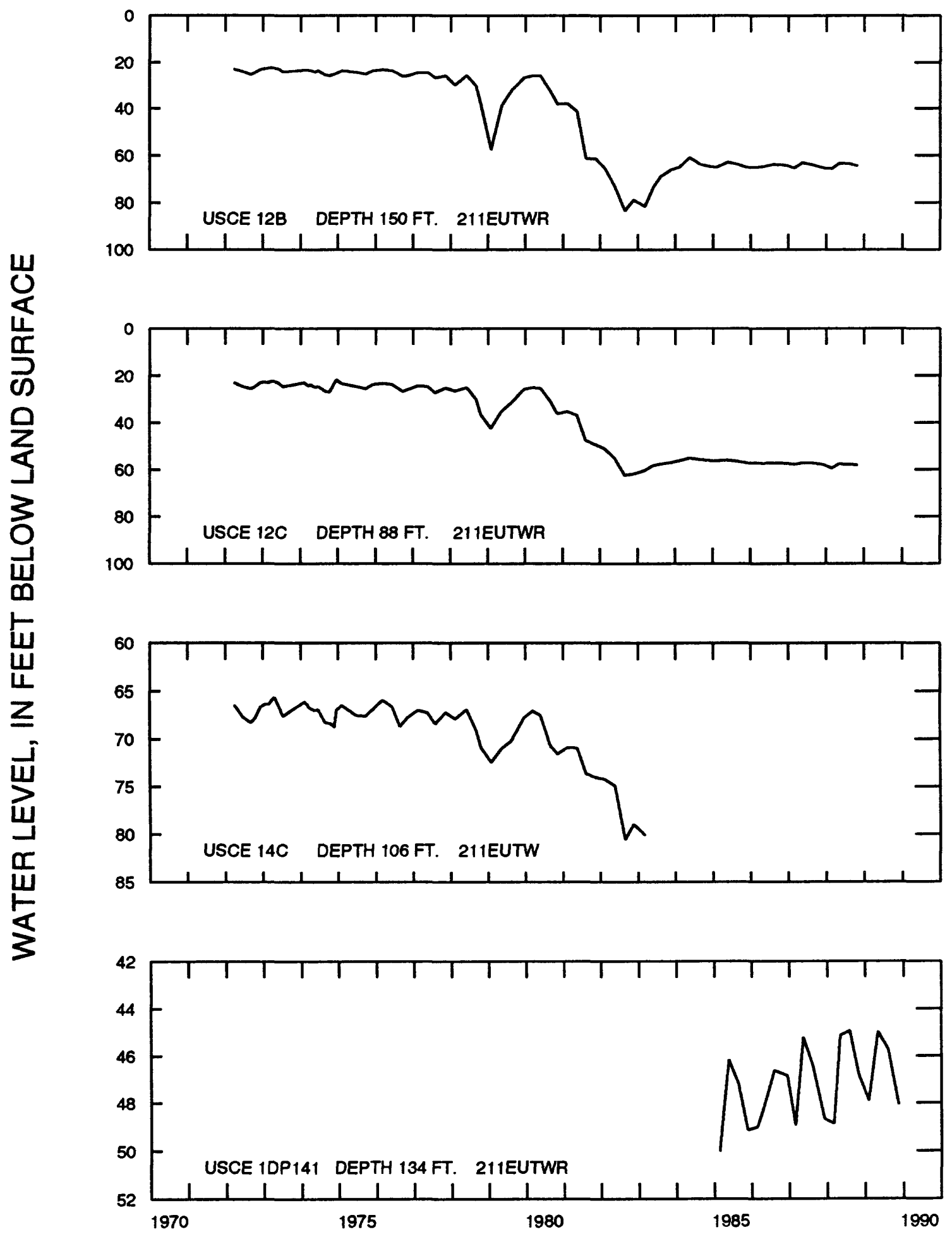

HYDROGRAPHS OF TENNESSEE-TOMBIGBEE OBSERVATION WELLS 

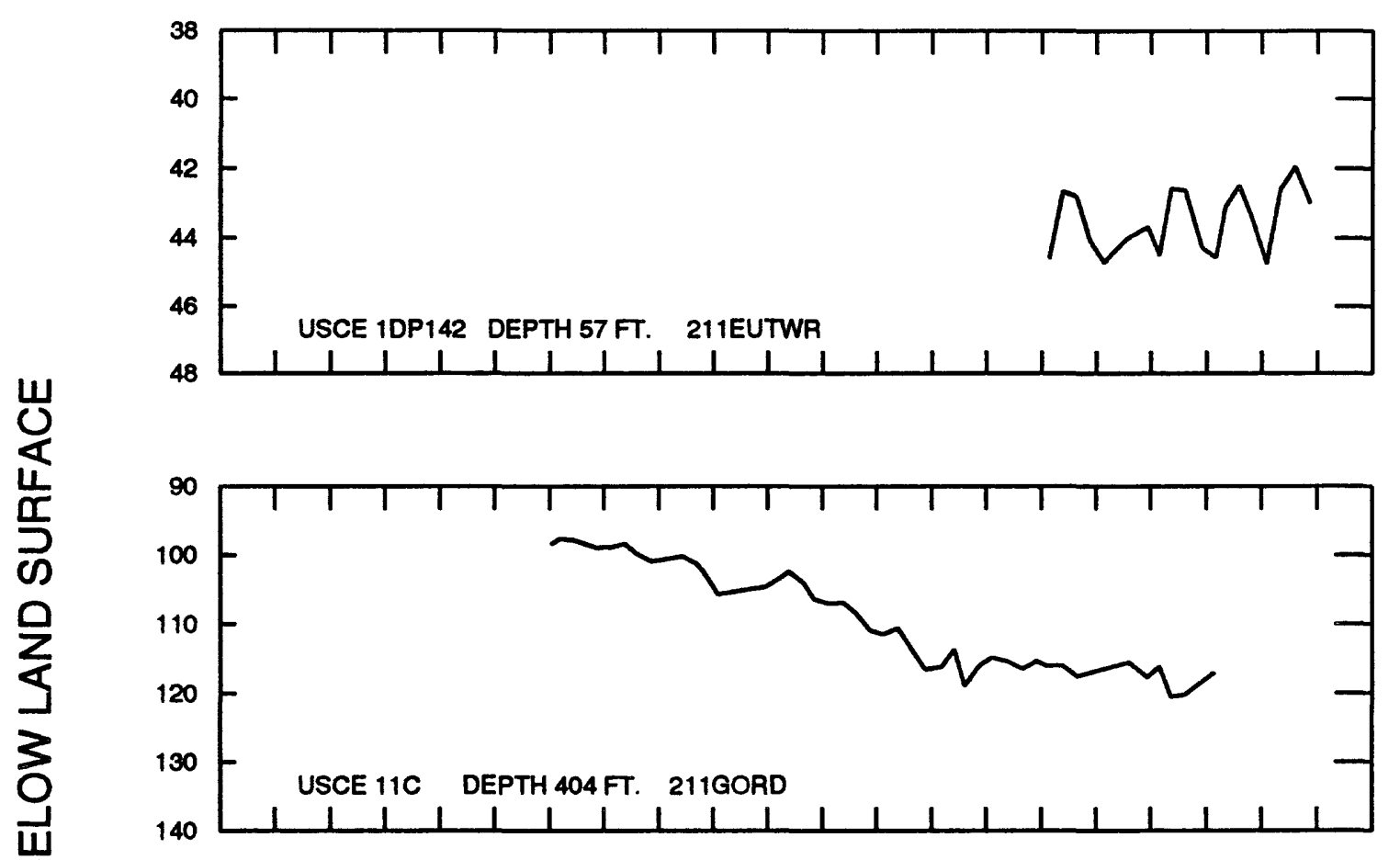

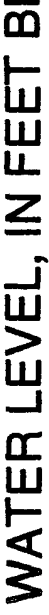

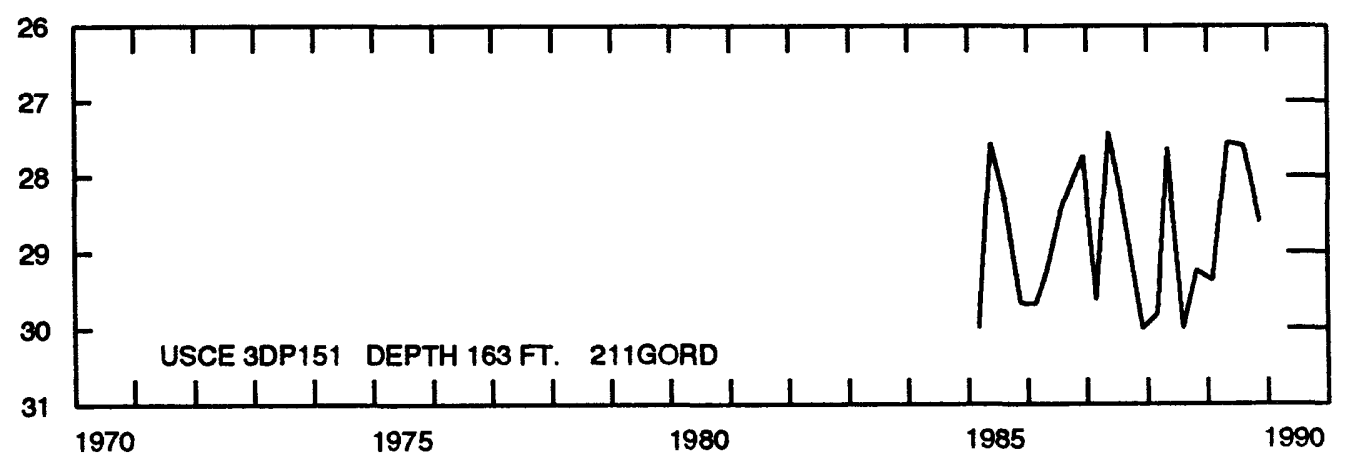

HYDROGRAPHS OF TENNESSEE-TOMBIGBEE OBSERVATION WELLS 


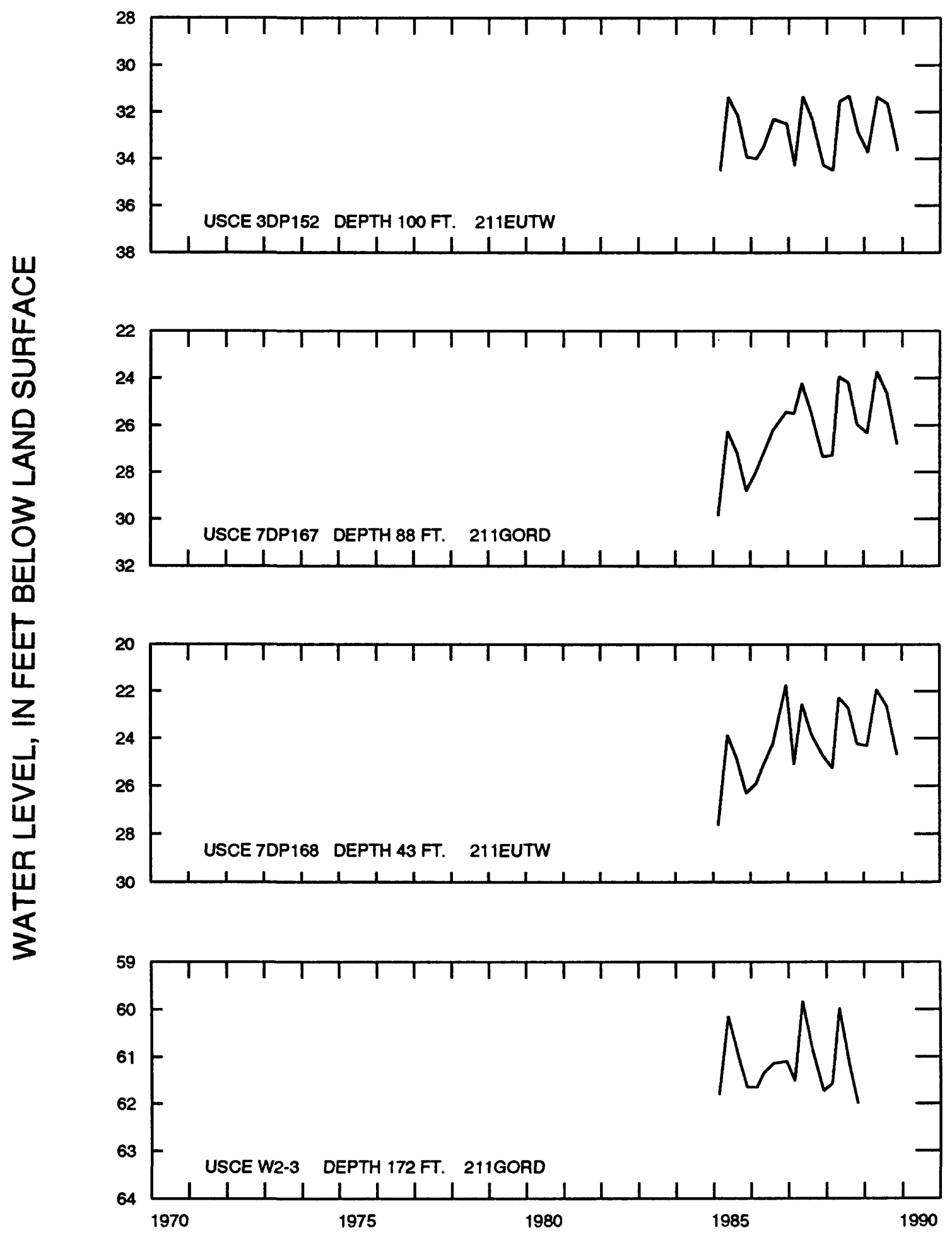

HYDROGRAPHS OF TENNESSEE-TOMBIGBEE OBSERVATION WELLS 


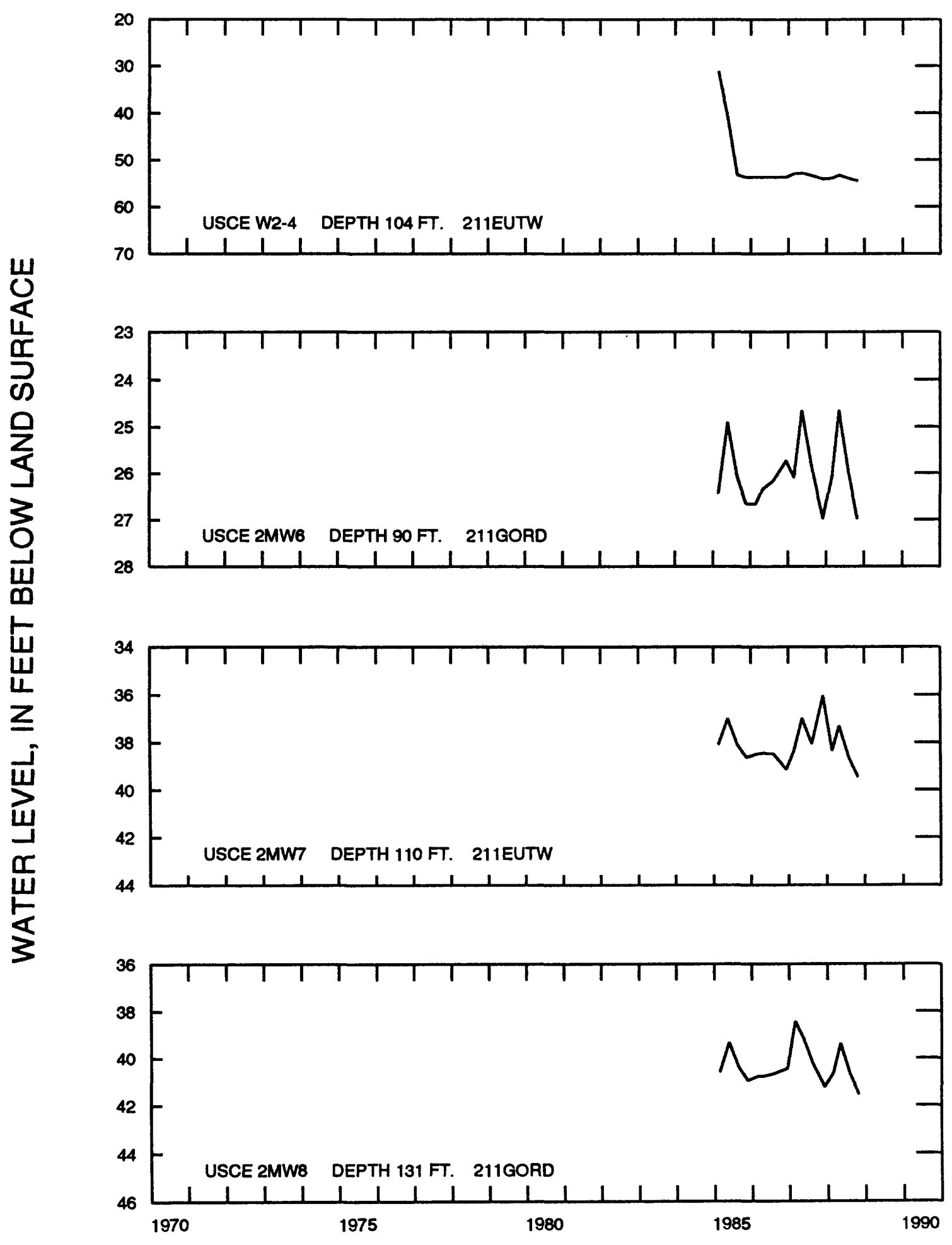

HYDROGRAPHS OF TENNESSEE-TOMBIGBEE OBSERVATION WELLS 


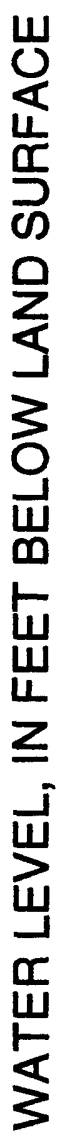

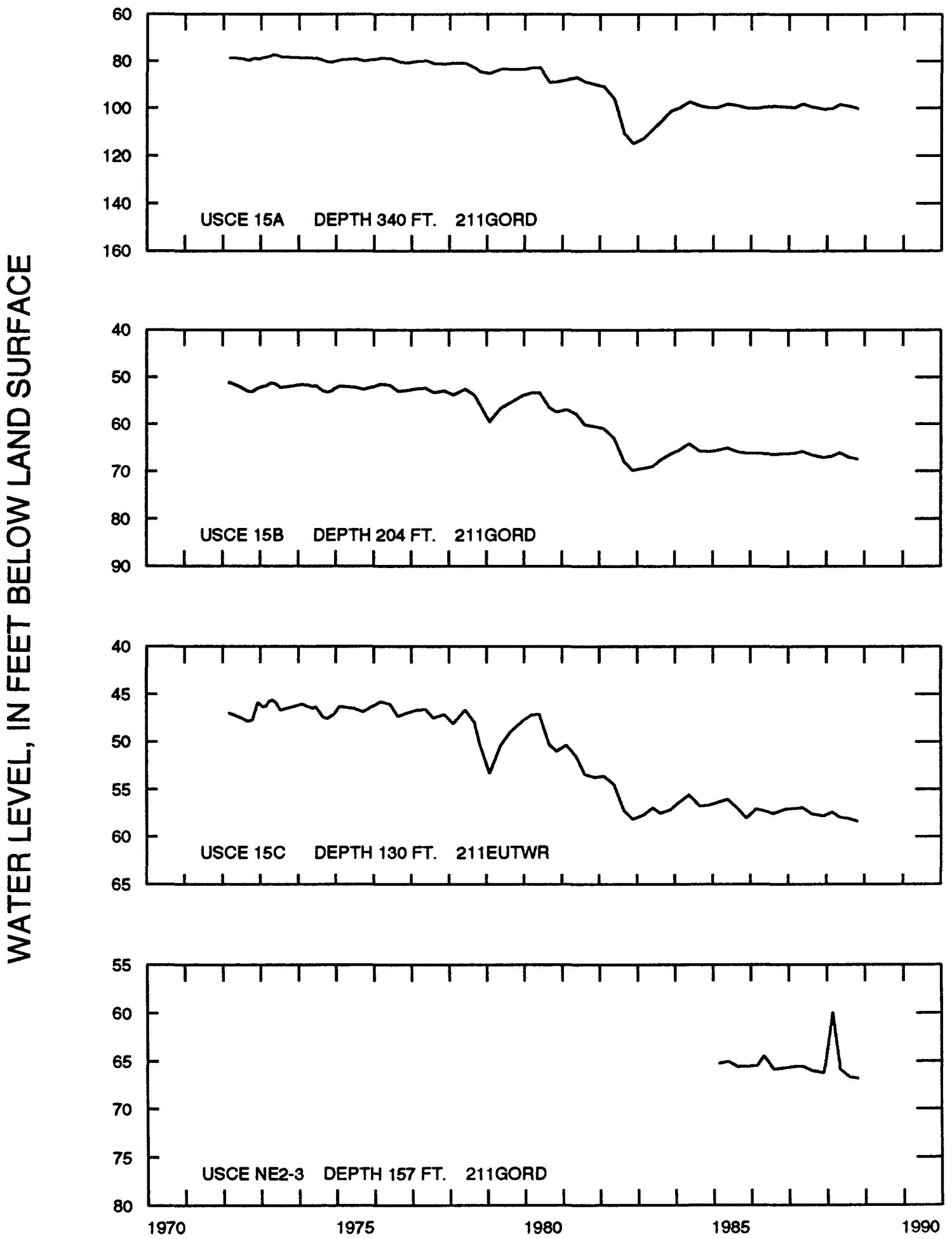

HYDROGRAPHS OF TENNESSEE-TOMBIGBEE OBSERVATION WELLS 


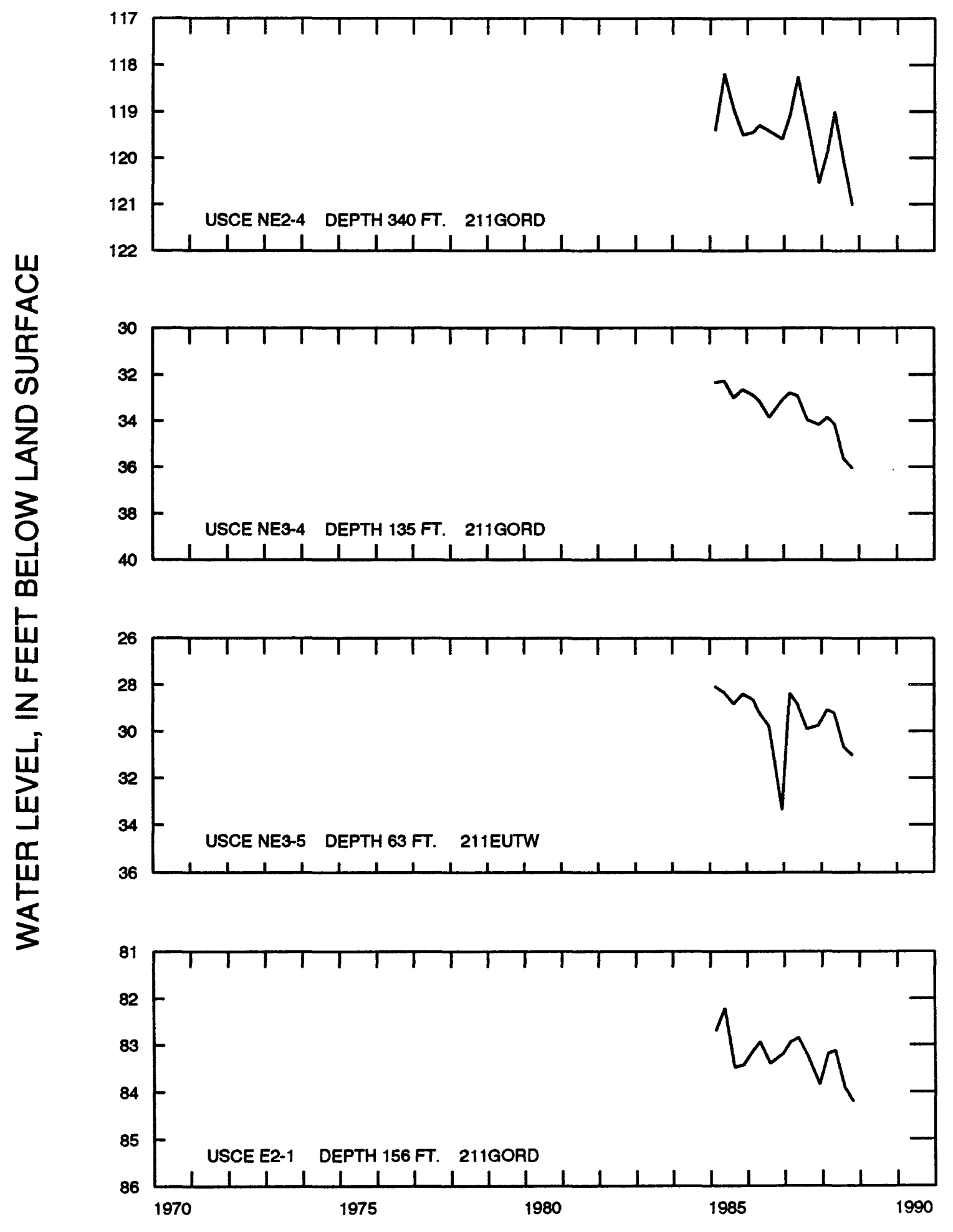

HYDROGRAPHS OF TENNESSEE-TOMBIGBEE OBSERVATION WELLS 


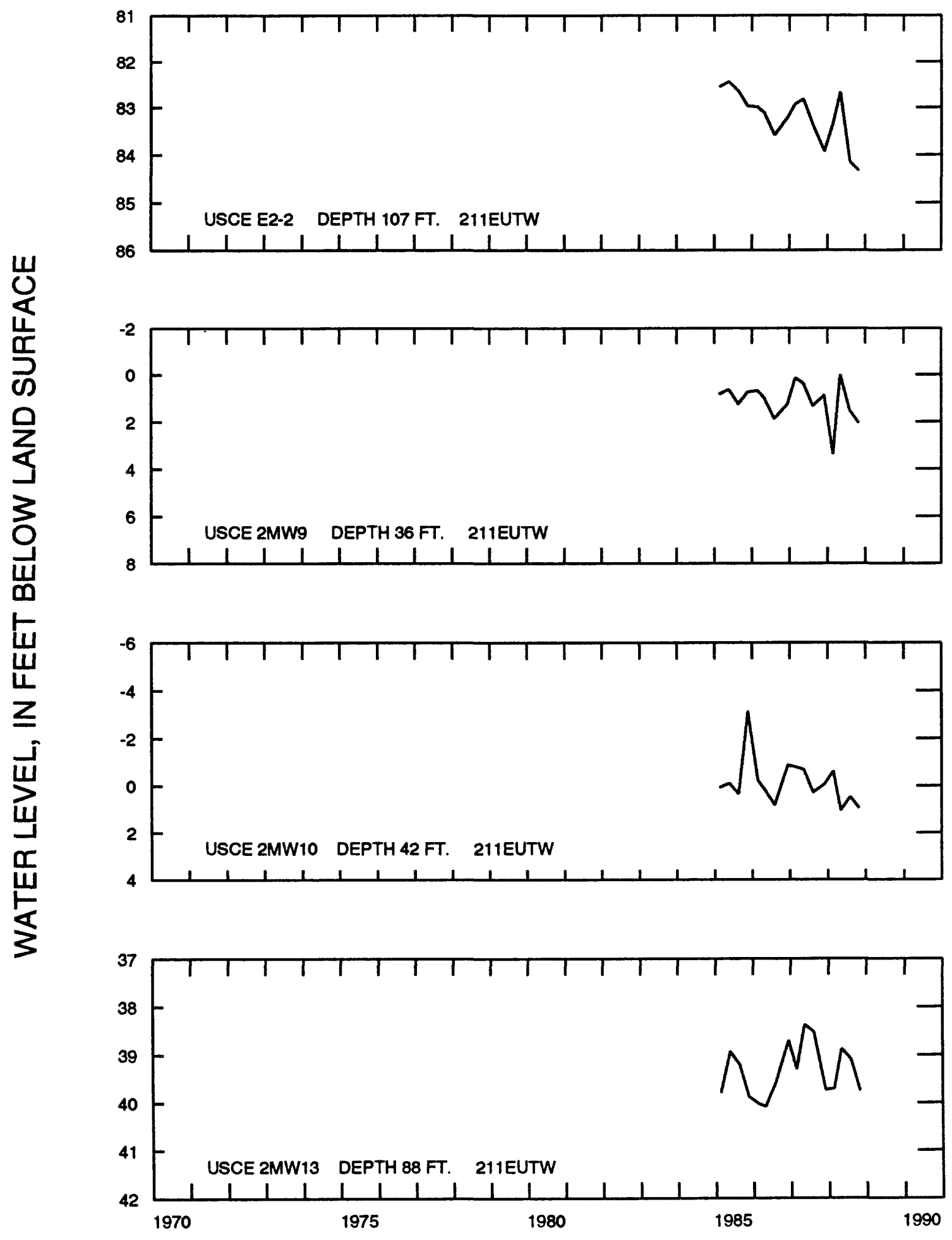

HYDROGRAPHS OF TENNESSEE-TOMBIGBEE OBSERVATION WELLS 


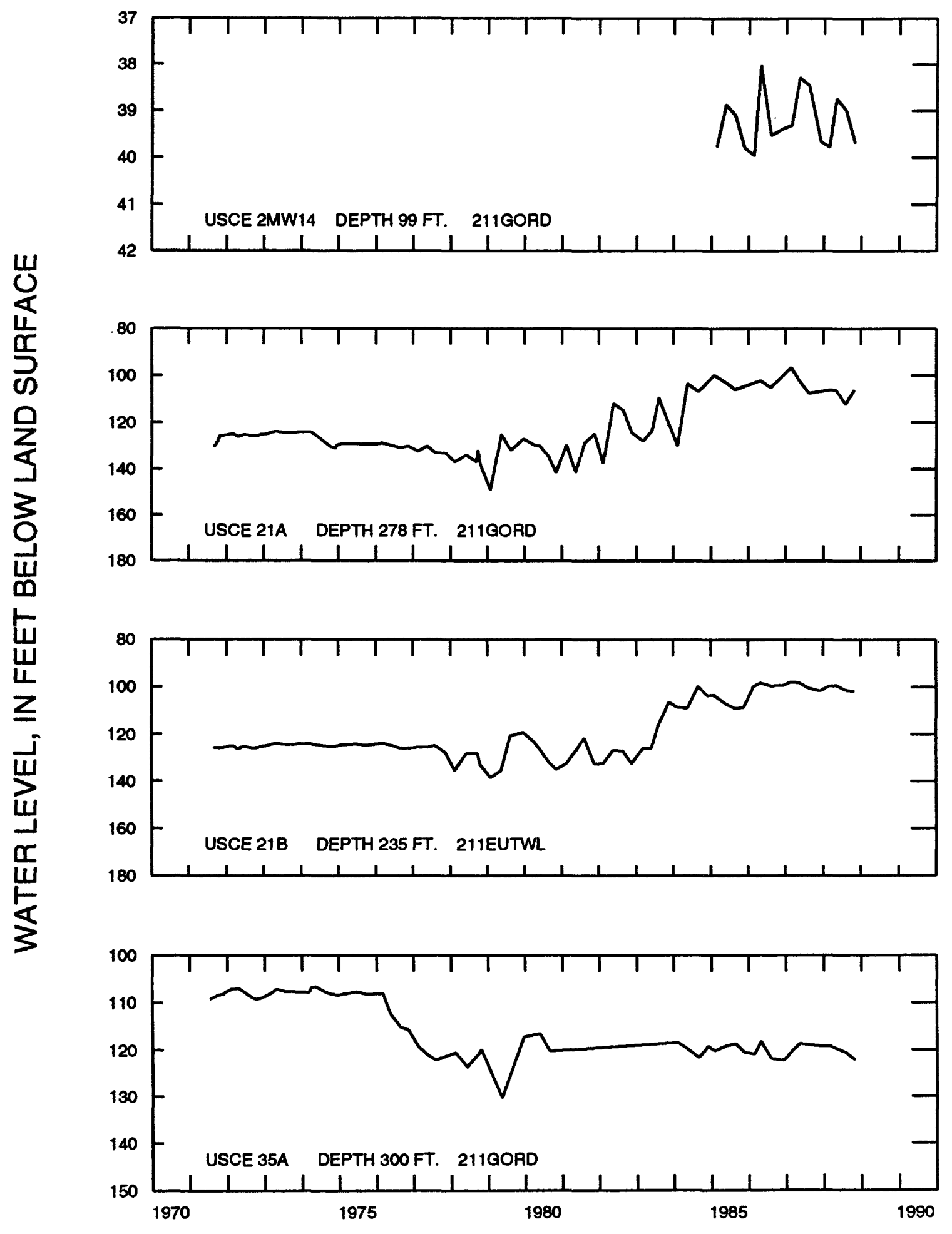

HYDROGRAPHS OF TENNESSEE-TOMBIGBEE OBSERVATION WELLS 


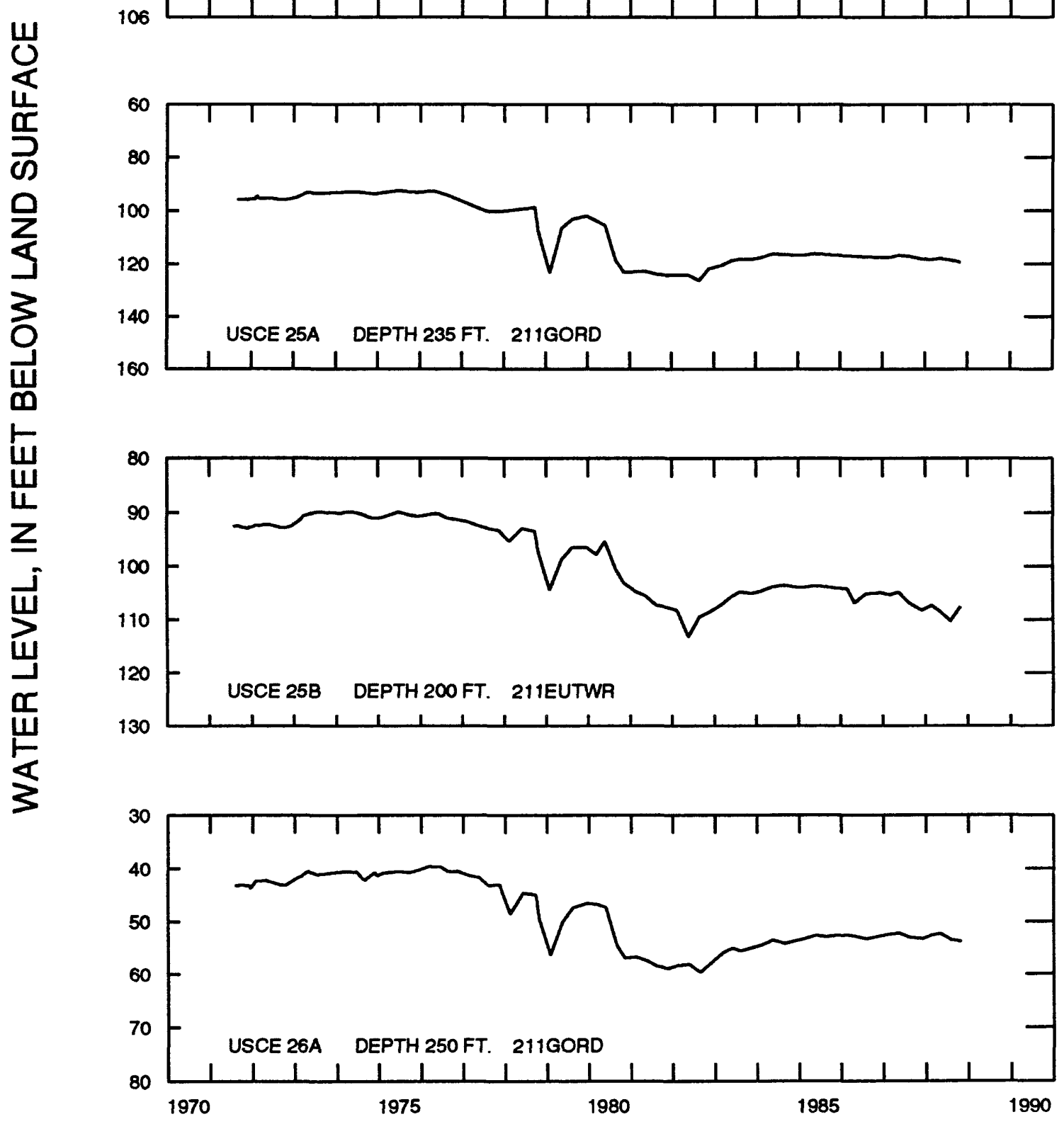

HYDROGRAPHS OF TENNESSEE-TOMBIGBEE OBSERVATION WELLS 


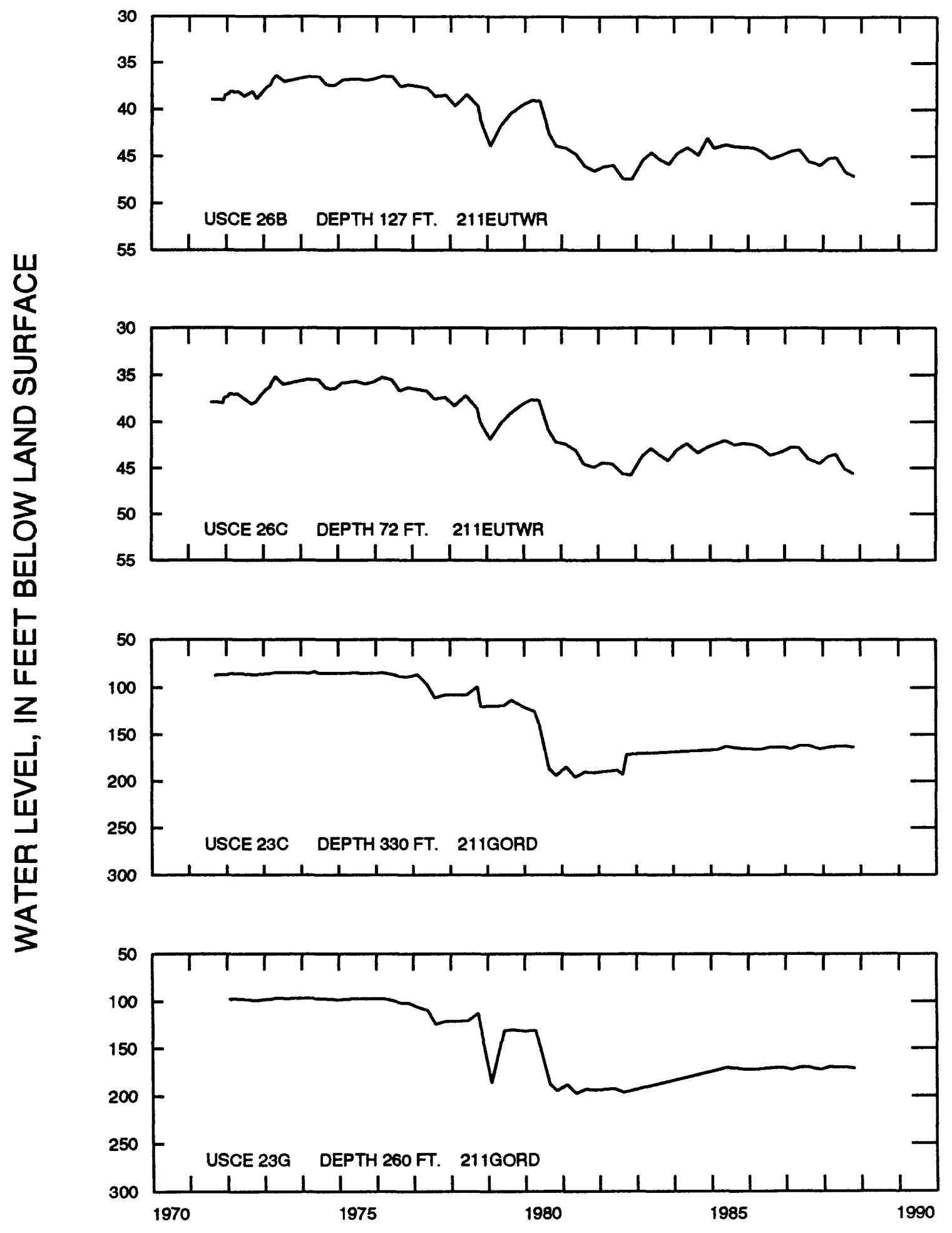

HYDROGRAPHS OF TENNESSEE-TOMBIGBEE OBSERVATION WELLS 


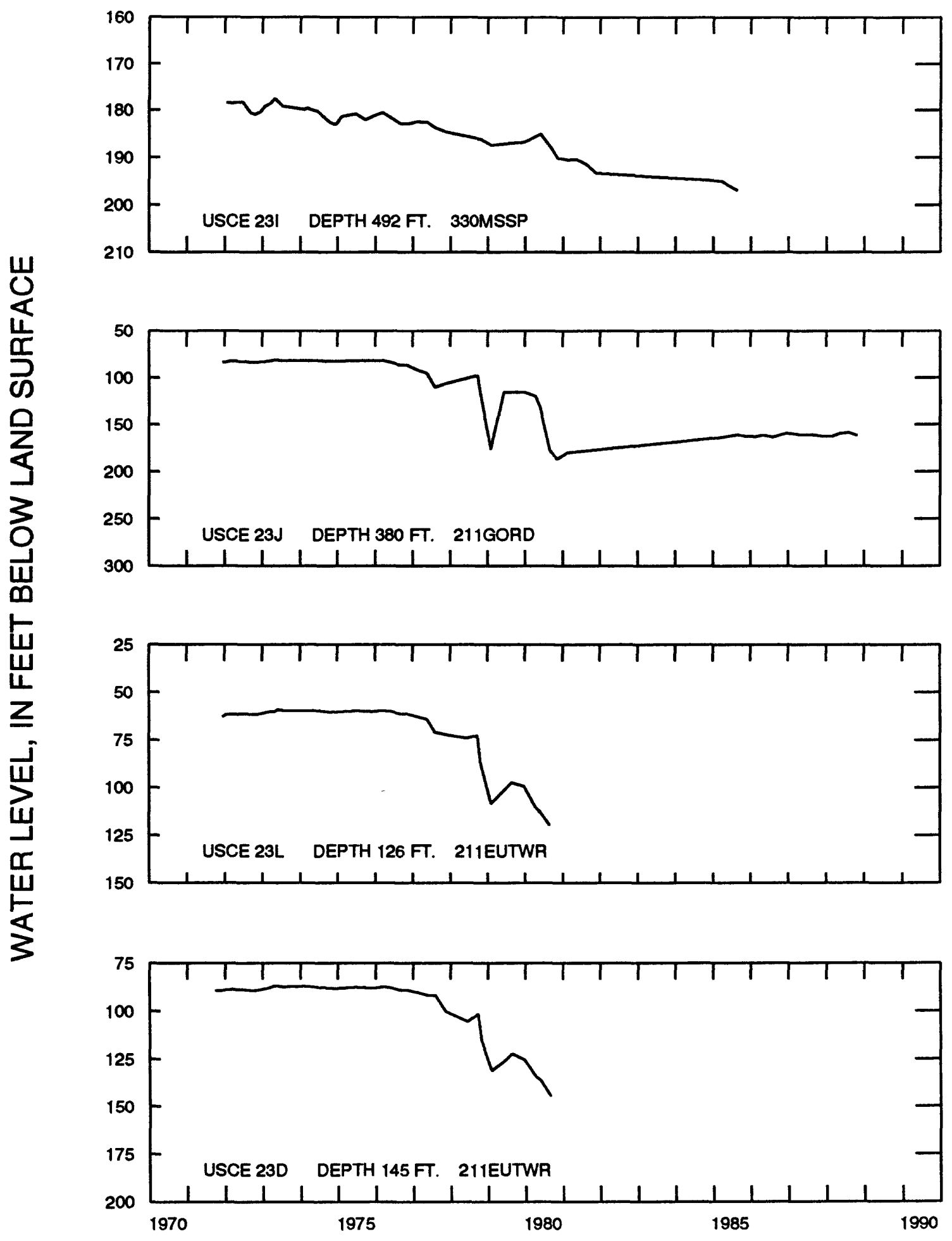

HYDROGRAPHS OF TENNESSEE-TOMBIGBEE OBSERVATION WELLS 


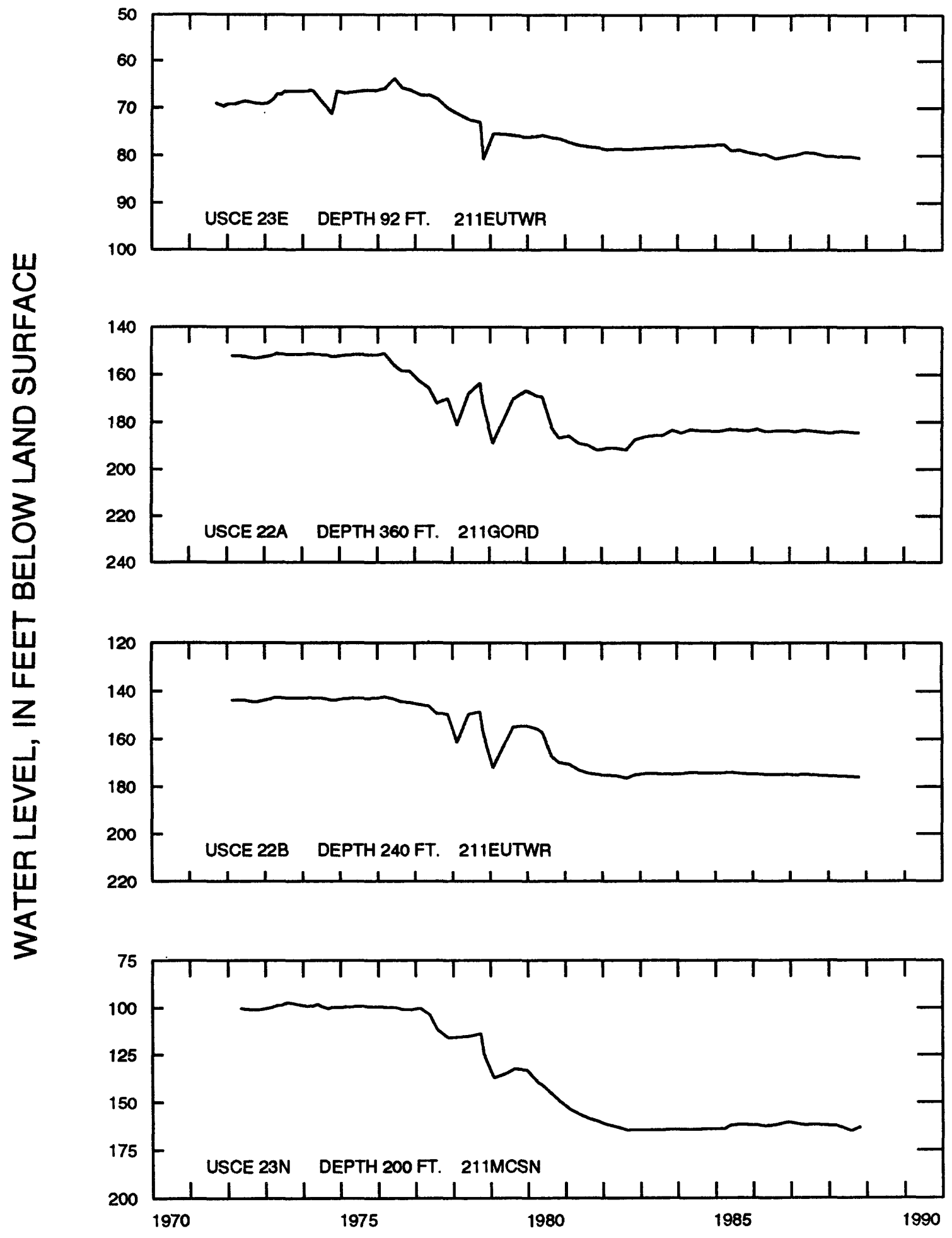

HYDROGRAPHS OF TENNESSEE-TOMBIGBEE OBSERVATION WELLS 


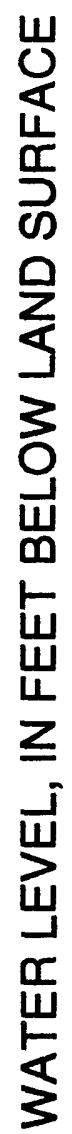
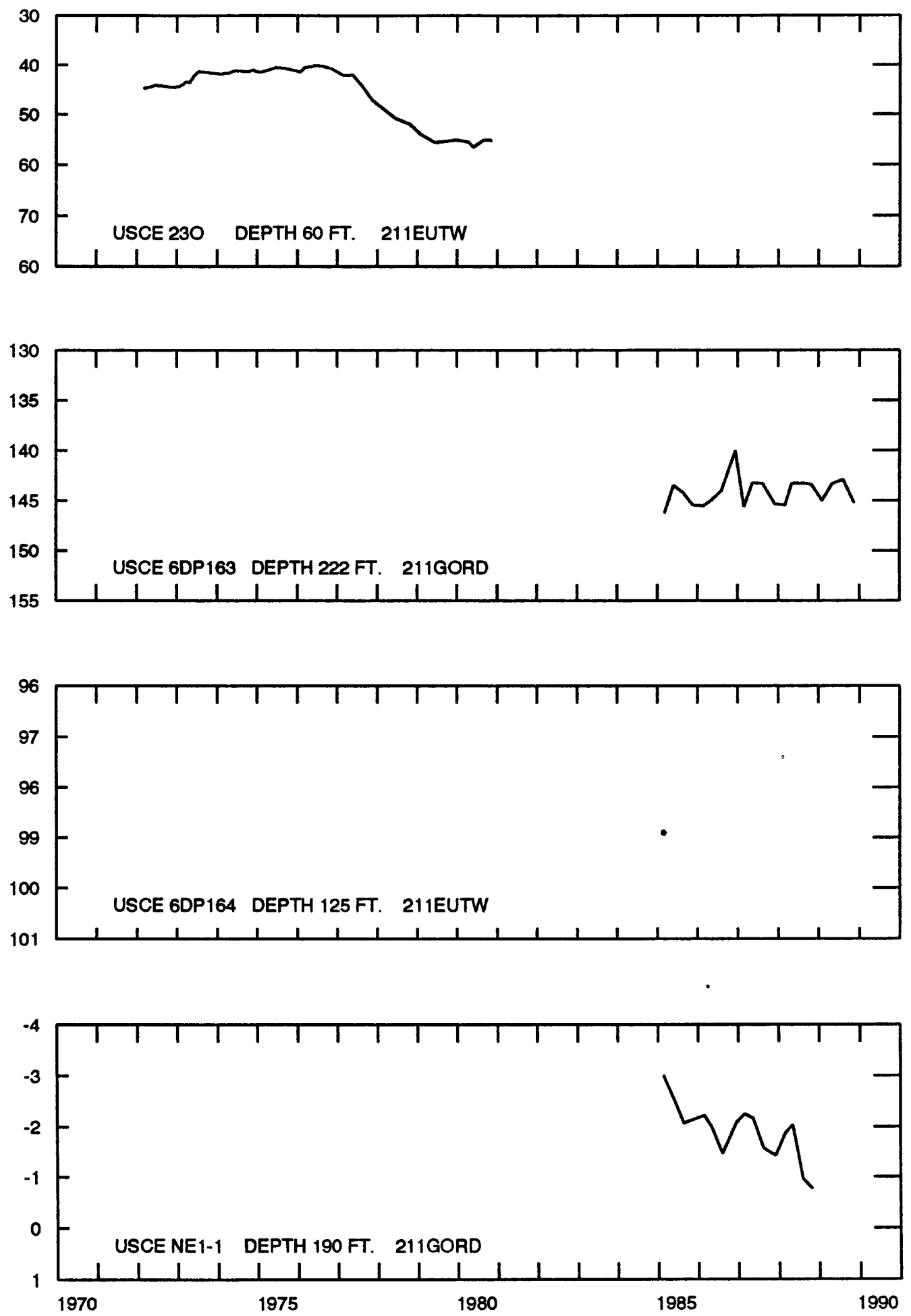

HYDROGRAPHS OF TENNESSEE-TOMBIGBEE OBSERVATION WELLS 


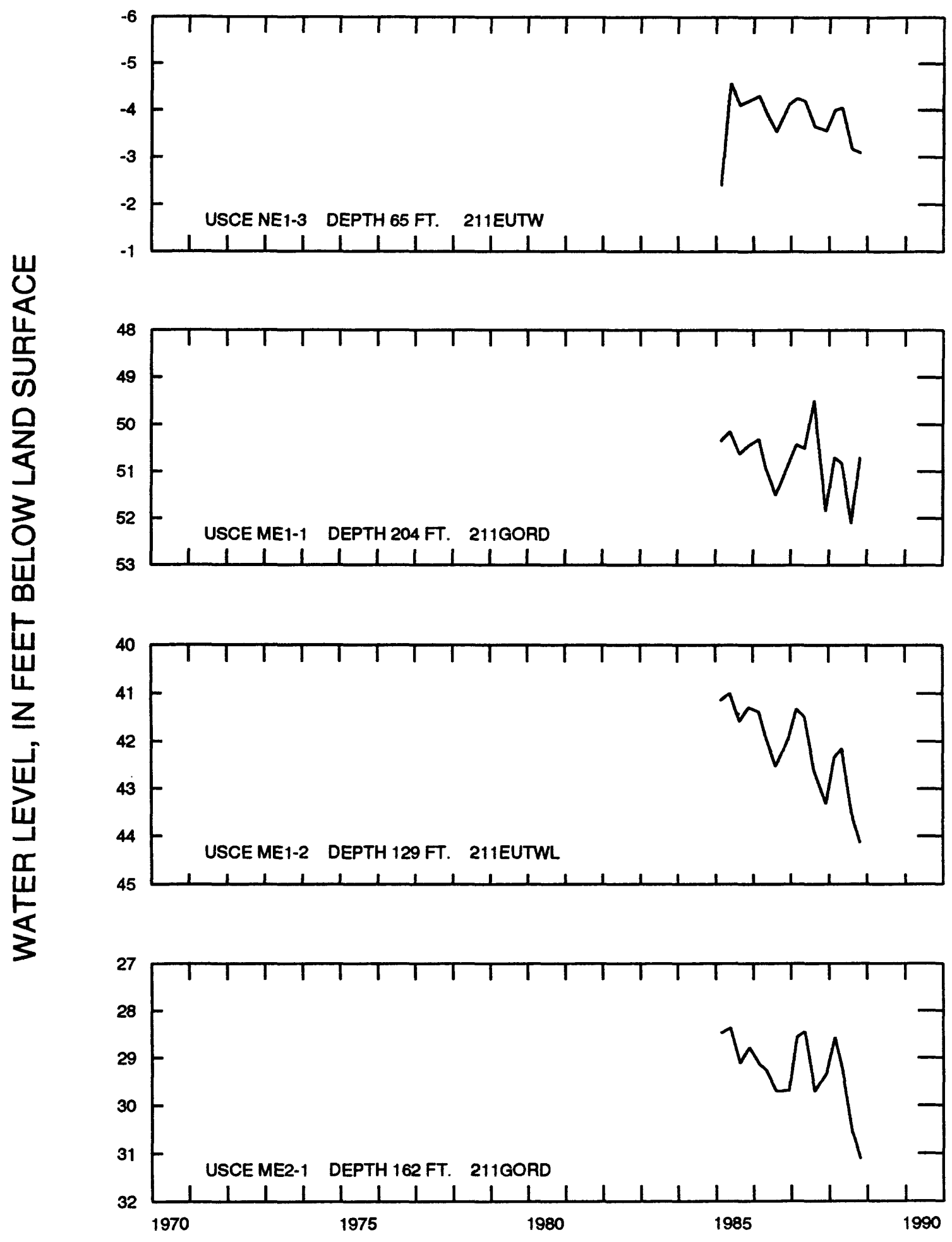

HYDROGRAPHS OF TENNESSEE-TOMBIGBEE OBSERVATION WELLS 


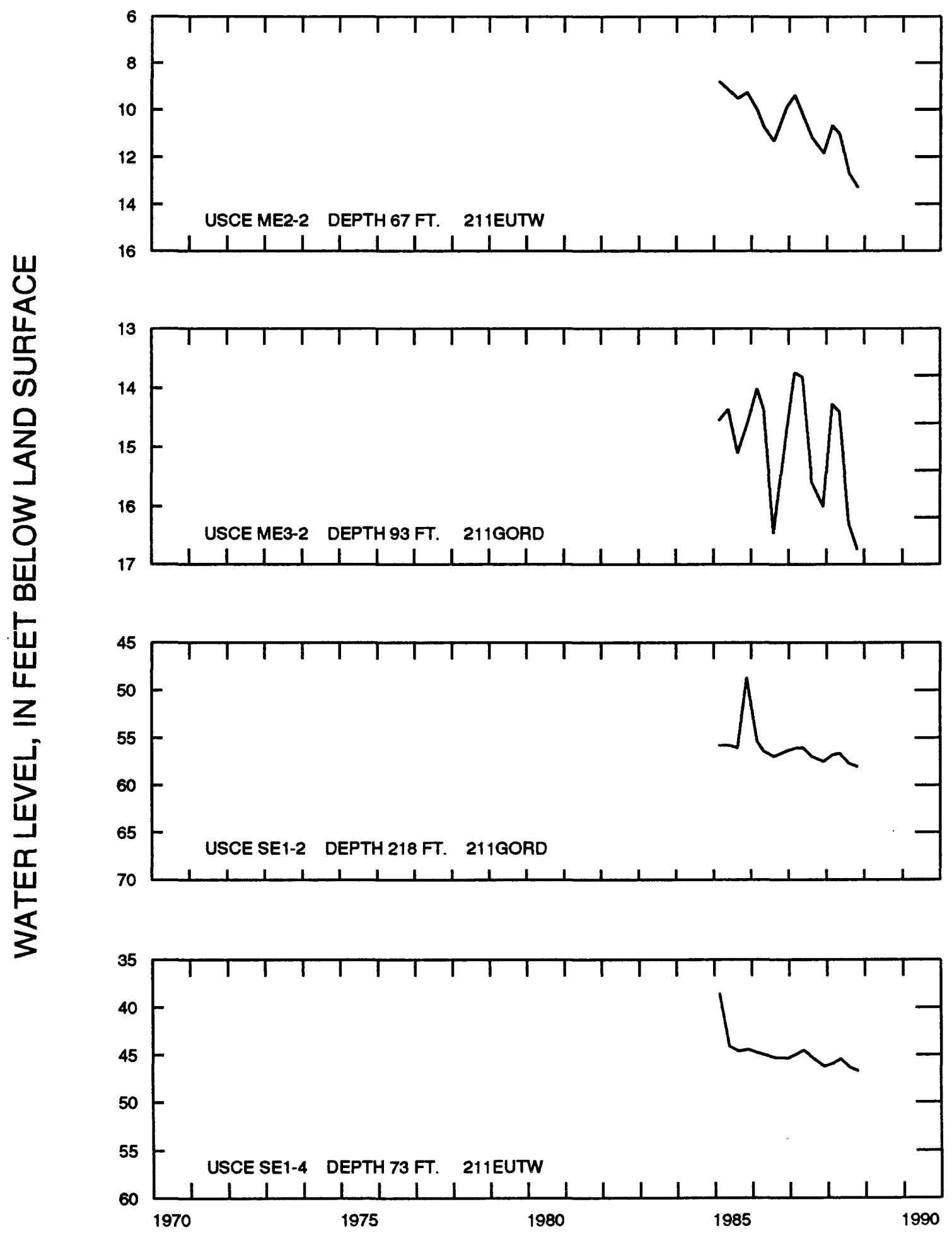

HYDROGRAPHS OF TENNESSEE-TOMBIGBEE OBSERVATION WELLS 


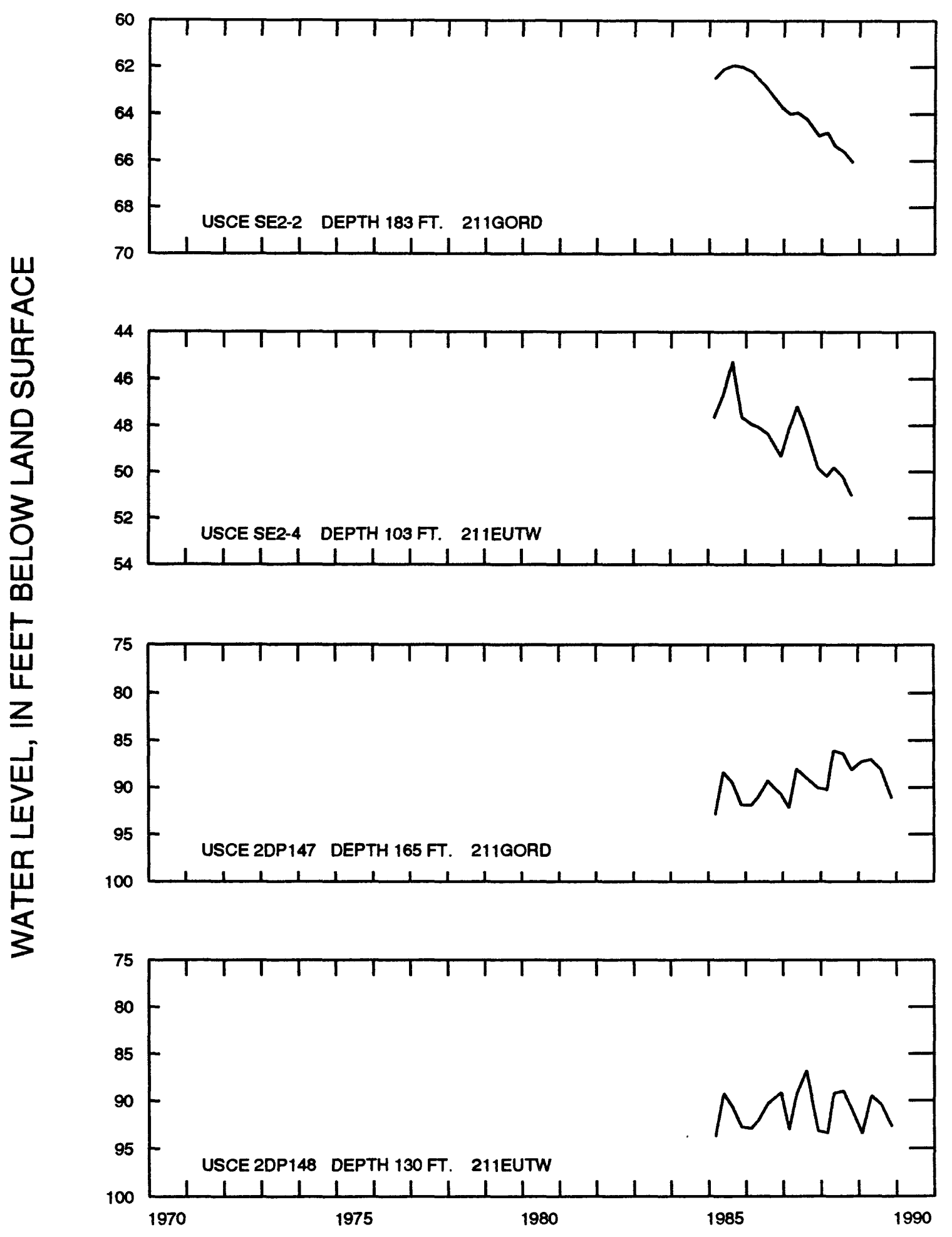

HYDROGRAPHS OF TENNESSEE-TOMBIGBEE OBSERVATION WELLS 


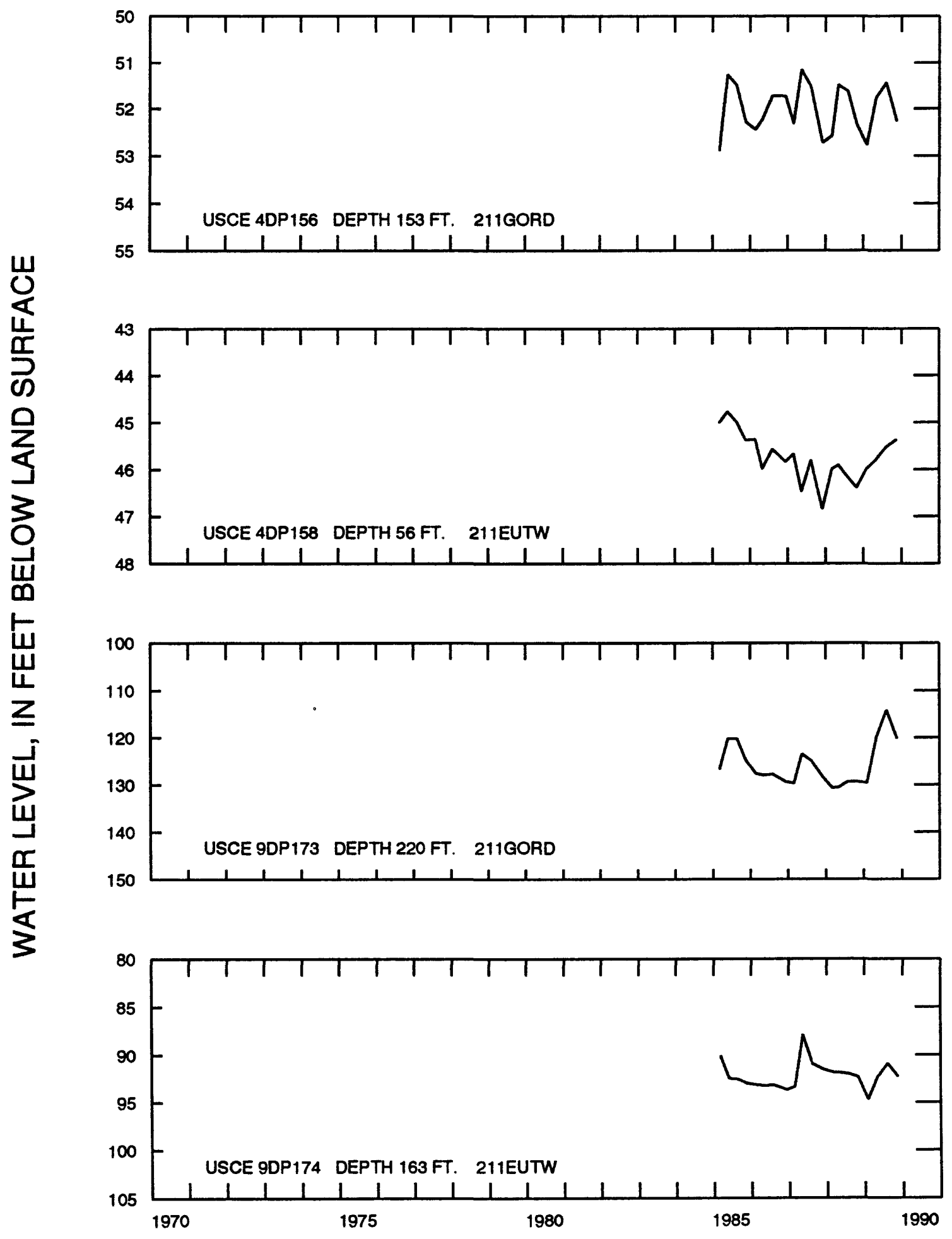

HYDROGRAPHS OF TENNESSEE-TOMBIGBEE OBSERVATION WELLS 


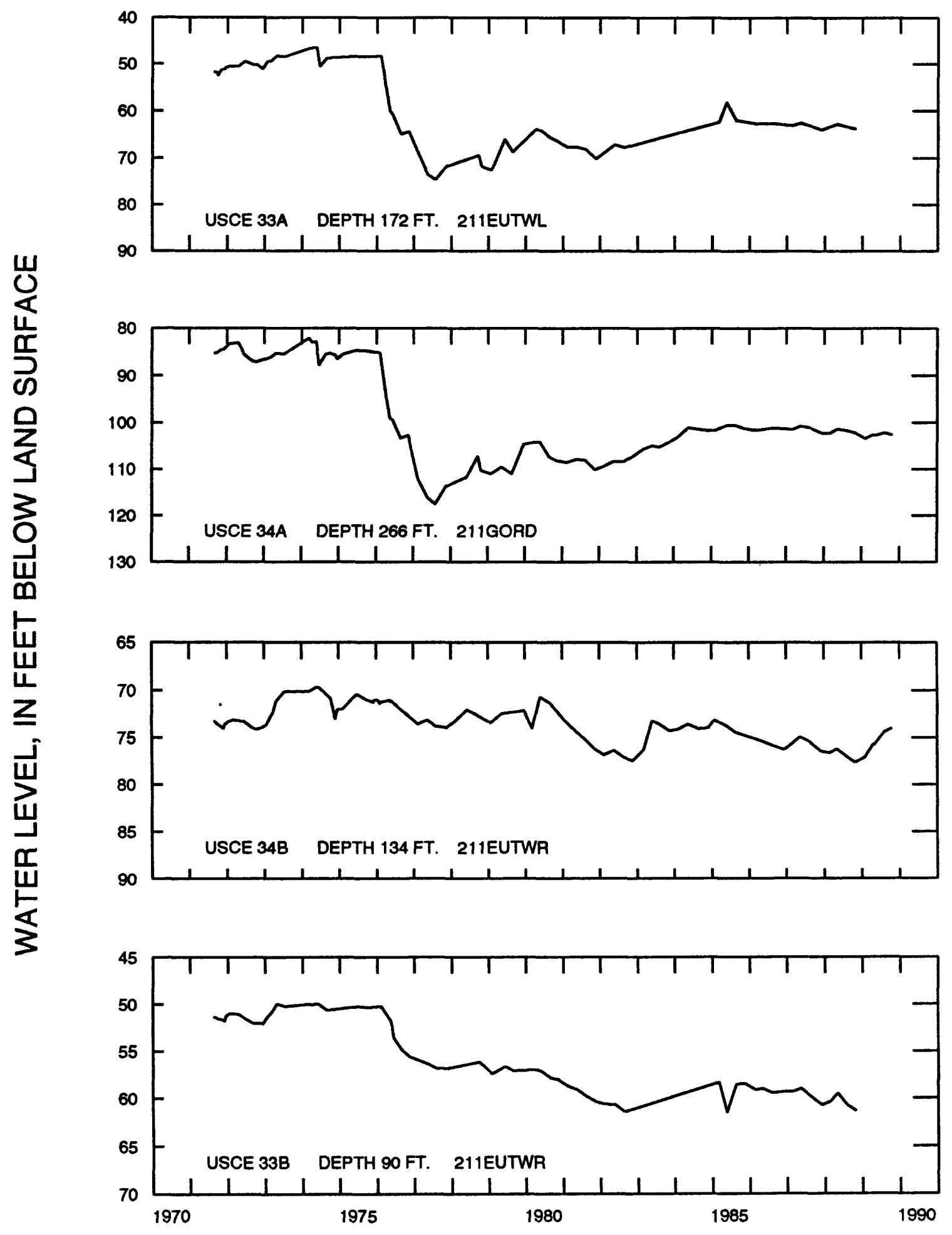

HYDROGRAPHS OF TENNESSEE-TOMBIGBEE OBSERVATION WELLS 


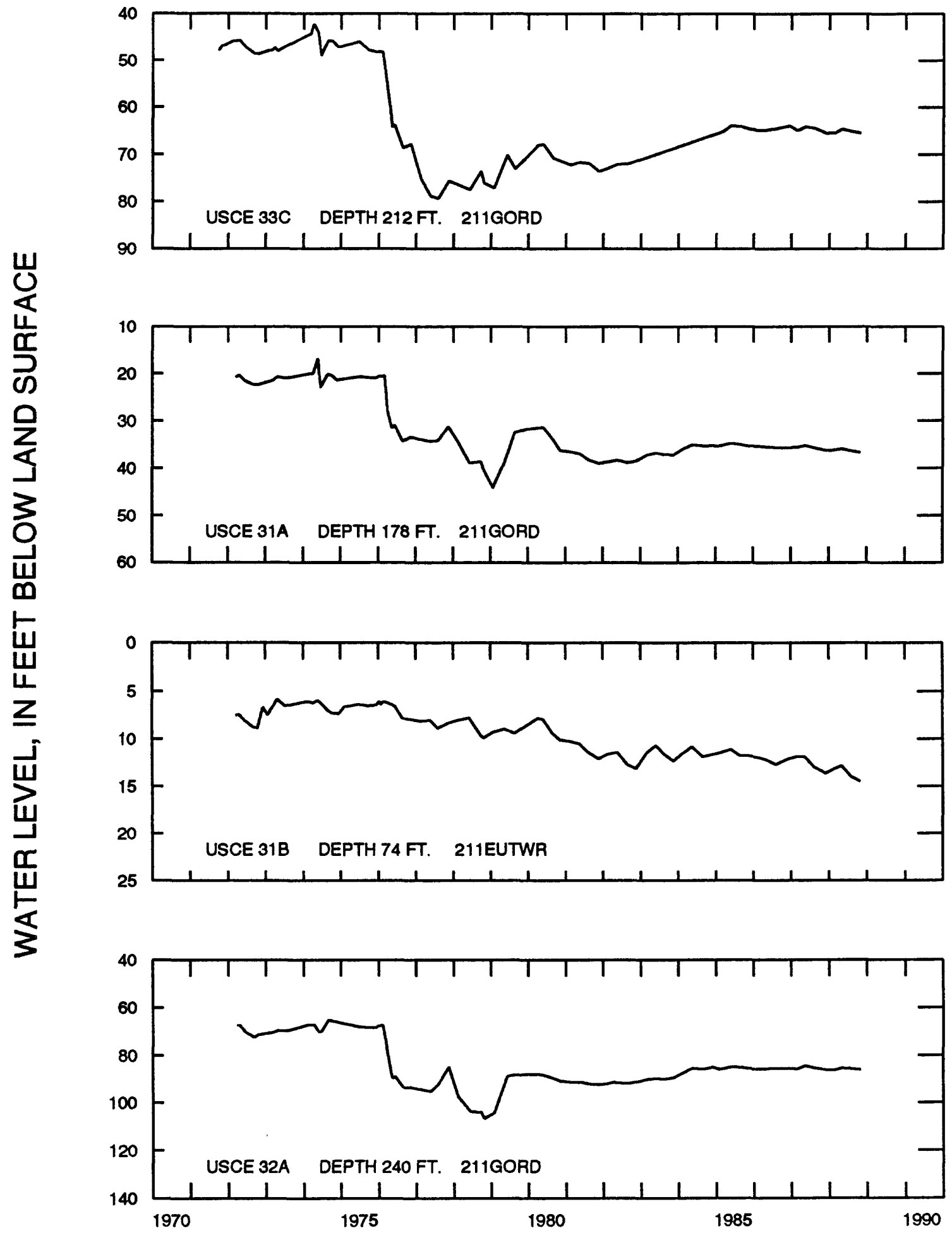

HYDROGRAPHS OF TENNESSEE-TOMBIGBEE OBSERVATION WELLS 


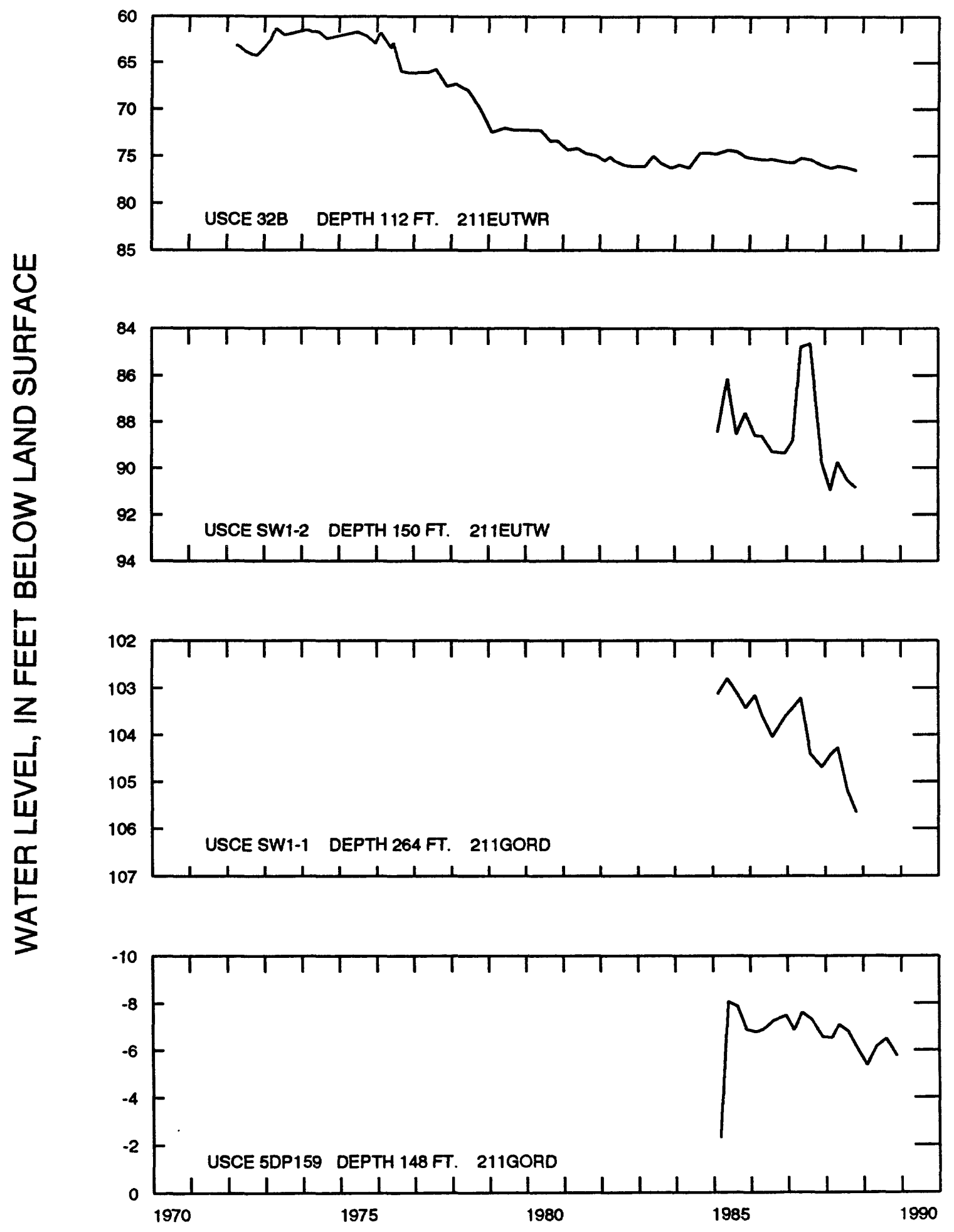

HYDROGRAPHS OF TENNESSEE-TOMBIGBEE OBSERVATION WELLS 


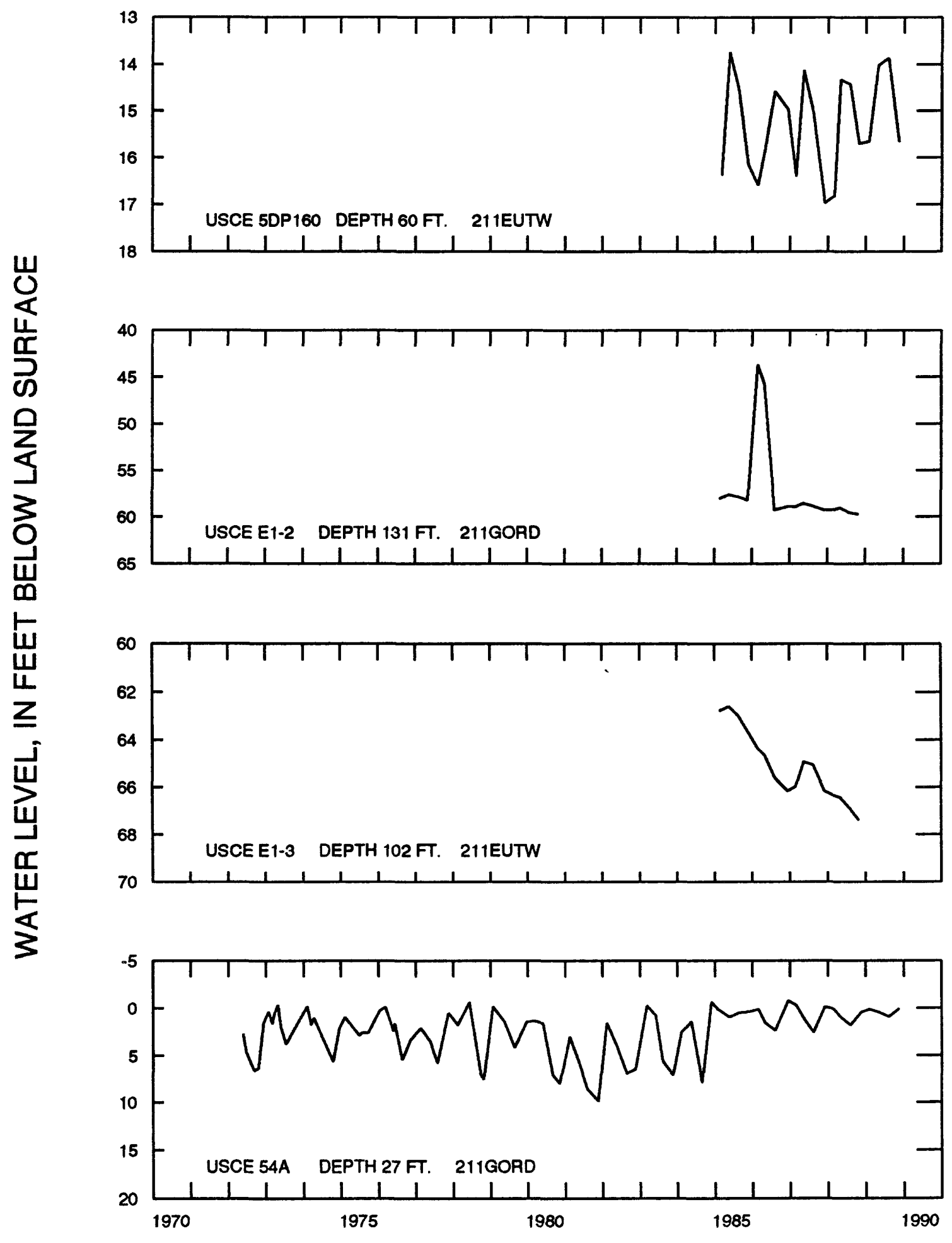

HYDROGRAPHS OF TENNESSEE-TOMBIGBEE OBSERVATION WELLS 


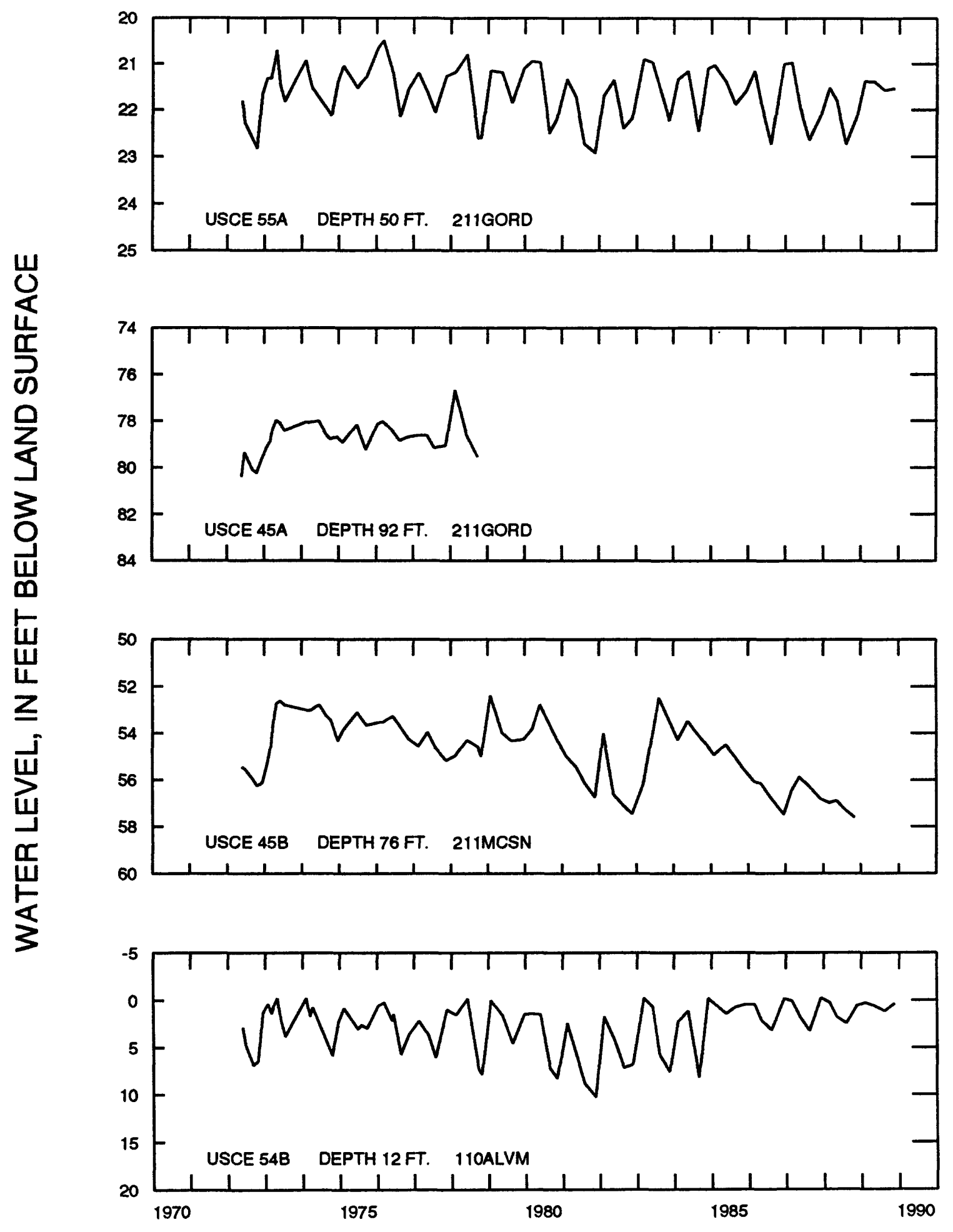

HYDROGRAPHS OF TENNESSEE-TOMBIGBEE OBSERVATION WELLS 


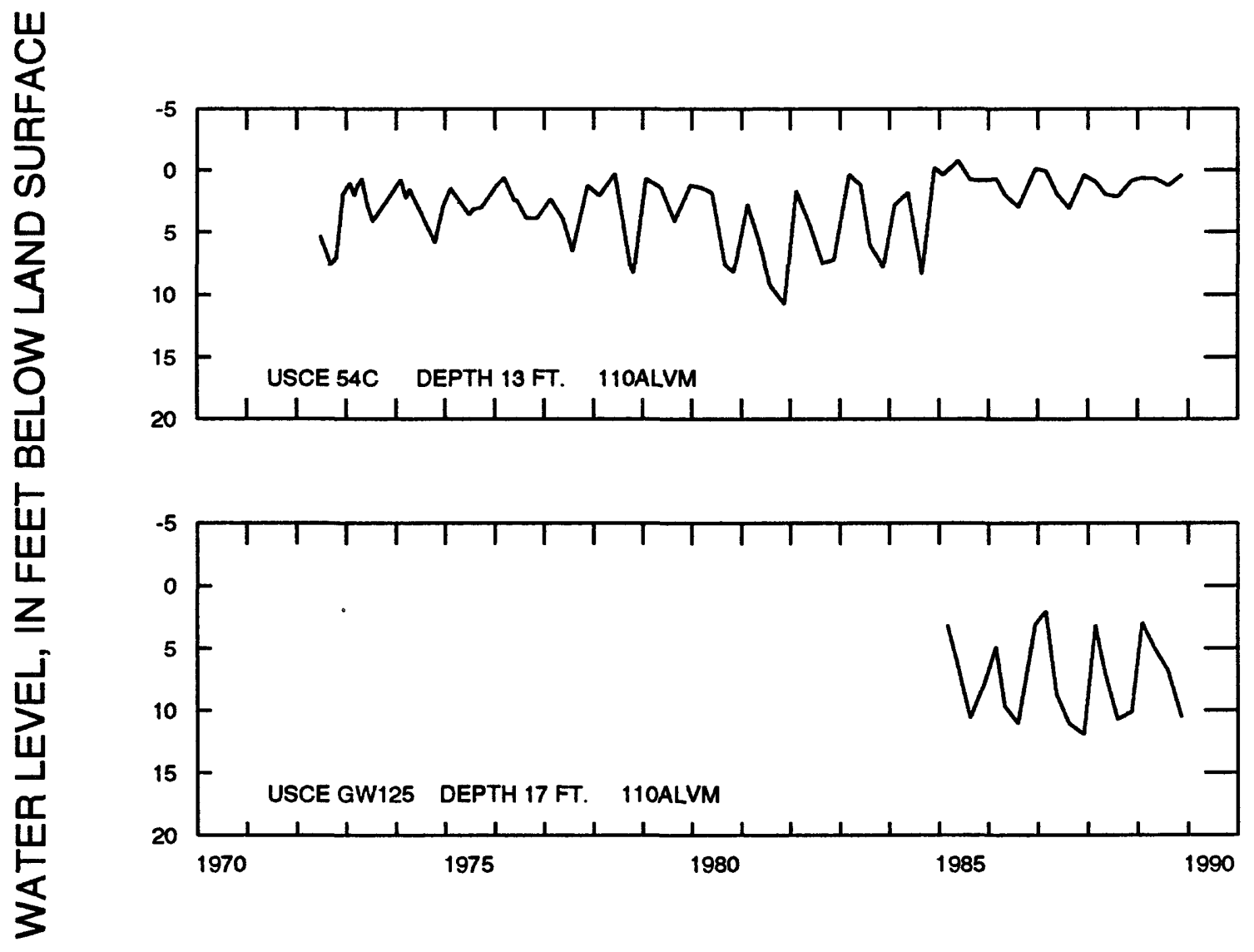

HYDROGRAPHS OF TENNESSEE-TOMBIGBEE OBSERVATION WELLS

101

PAGE 103 FOLLOW'S 
APPENDIX B

SURFACE-WATER DATA

103

PAGE 105 FOLLOWS 


\section{APPENDIX B \\ SURFACE-WATER DATA}

DESCRIPTIONS OF SITES

105

PAGE 107 FOLLOWS 
DRAIN-

SURFACE-WATER NETWORK

03592824 02433000 02436500 333927088304935 02439600

02441000 02441400 02441498 02443500 02443610

02444210 02446500 02447010 02448500 02467001

02430500 02430680 02430690 02431000 02431410

02433500 02437000 02437500 02439400 332929088273300

332751088261000 3321120882235 3321000882248 3320300882122 02444157

02444500 02447008 325645088100700 02448000 02449000

323704087542400 323653087540800 323642087541800 02469762
TENNESSEE-TOMBIGBEE WATERWAY AT CROSS ROADS, MS BULL MOUNTAIN CREER NEAR SMITHVILLE, MS

TOWN CREEK NEAR NETTLETON, MS

TTW COLUMBUS LAKE BUTTAHATCHEE RIVER BEND SR 26A BUTTAHATCHEE RIVER NEAR KOLOLA SPRINGS, MS

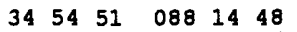
$\begin{array}{llllll}34 & 05 & 18 & 088 & 23 & 26\end{array}$ $\begin{array}{llllll}34 & 03 & 32 & 088 & 37 & 40\end{array}$ $\begin{array}{llllll}33 & 39 & 27 & 088 & 30 & 49\end{array}$ $\begin{array}{llllll}33 & 40 & 24 & 088 & 25 & 45\end{array}$

$\begin{array}{llllll}33 & 32 & 17 & 088 & 38 & 00\end{array}$ TIBBEE CREEK NEAR TIBBEE, MS $\begin{array}{llllll}33 & 29 & 40 & 088 & 27 & 40\end{array}$ TOMBIGBEE RIVER NEAR COLUMBUS, MS IUXAPALIIIA CREER NEAR COLUMBUS, MS AT COLUMBUS, MS 33230500882342

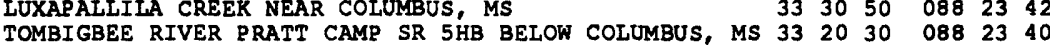
TOMBIGBEE RIVER BIG CREEK BEND NEAR PICKENSVILLE, AL SIR SEY RIVER NEAR ELROD, AL TOMBIGBEE RIVER COOKS BEND NEAR WARSAW, AI NOXUBEE RIVER NEAR GEIGER, AL

$\begin{array}{llllll}33 & 11 & 11 & 088 & 16 & 03 \\ 33 & 15 & 25 & 087 & 46 & 35 \\ 32 & 57 & 38 & 088 & 11 & 14 \\ 32 & 55 & 06 & 088 & 17 & 45 \\ 32 & 31 & 15 & 087 & 52 & 48\end{array}$ TOMBIGBEE RIVER BELOW DEMOPOLIS LOCK AND DAM, AL SITES NOT IN THE SURFACE-WATER NETWORK

\section{TOMBIGBEE RIVER NEAR MARIETTA, MS} TWENTYMILE CREEK NEAR GUNTOWN, MS TWSNTYMILE CREEK NEAR MANTACHIE, MS TOMBIGBEE RIVER NEAR FULTON, MS MANTACHIE CREEK BELOW DORSEY, MS

TOMBIGBEE RIVER AT BIGBEE, MS TOMBIGBEE RIVER NEAR AMORY, MS TOMBIGBEE RIVER AT ABERDEEN, MS BUTTAHATCHEE RIVER NEAR ABERDEEN, MS

TTW ALIVEVIILE LAKE ABOVE COLUMBUS BEND

TTK ALICEVILLE ILAKE COLUMBUS CUT

TTW ALICEVILIE LAKE ABOVE HAIRSTON BEND

TTW ALICEVILLE LAKE HAIRSTON BEND SR $11 \mathrm{HB}$

TTW ALICEVILLE TAAKE HAIRSTON CUT

TOMBIGBEE RIVER NEAR COCHRANE, AI

$\begin{array}{llllll}33 & 04 & 56 & 088 & 14 & 16\end{array}$

TOMBIGBEE RIVER ABOVE COOKS BEND SR 5A NR WARSAW, AL $325722 \quad 0880945$

TTW GAINESVILIE IAKE COOKS BEND CUT SR 4AD $\quad 3256450881007$ NOXUBEE RIVER AT MACON, MS

TOMBIGBEE RIVER AT GAINESVILLE, AI

$\begin{array}{llllll}33 & 06 & 08 & 088 & 33 & 40\end{array}$

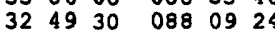

TTW DEMOPOLIS IAAKE ABOVE RATTLESNAKE BEND SR 12CA

TTW DEMOPOLIS LAKE RATTLESNAKE BEND SR RBI

$\begin{array}{lllllll}32 & 37 & 04 & 087 & 54 & 24\end{array}$

$\begin{array}{llllll}32 & 36 & 53 & 087 & 54 & 08\end{array}$

TTW DEMOPOIIS IAKE RATTIESNAKE CUT SR 12CB $\quad 323642 \quad 0875418$

TOMBIGBEE RIVER BELOW COFFEEVILLE LOCK AND DAM, AL $314530 \quad 0880735$
$00 \quad 06030005$

$00 \quad 03160101$

$00 \quad 03160102$

3503160101

$00 \quad 03160103$

620

855

$00 \quad 03160104 \quad 926$

$00 \quad 03160101 \quad 4450$

03160105

$03160105 \quad 715$

$00 \quad 03160106$

03160106

$03160107 \quad 528$

03160106

$03160108 \quad 1100$

0316020115400

$00 \quad 03160101 \quad 308$

$00 \quad 03160101 \quad 131$

$00 \quad 03160101 \quad 150$

$\begin{array}{lll}00 & 03160101 & 612 \\ 00 & 03160101 & 66.9\end{array}$

$00 \quad 03160101 \quad 1230$

$00 \quad 03160101 \quad 1930$

$00 \quad 03160101 \quad 2170$

$00 \quad 03160103 \quad 798$

03160101

03160101

03160106

03160106

03160106

$00 \quad 03160106$

$03160106 \quad 5940$

03160106

03160106

$00 \quad 03160108 \quad 768$

$00 \quad 03160106 \quad 8630$

03160106

03160106

03160106

0316020318400 


\section{APPENDIX B}

\section{SURFACE-WATER DATA}

WATER-QUALITY ANALYSES

109 
03592824 TENNESSEE-TOMBIGBEE WATERWAY AT CROSS ROADS, MS

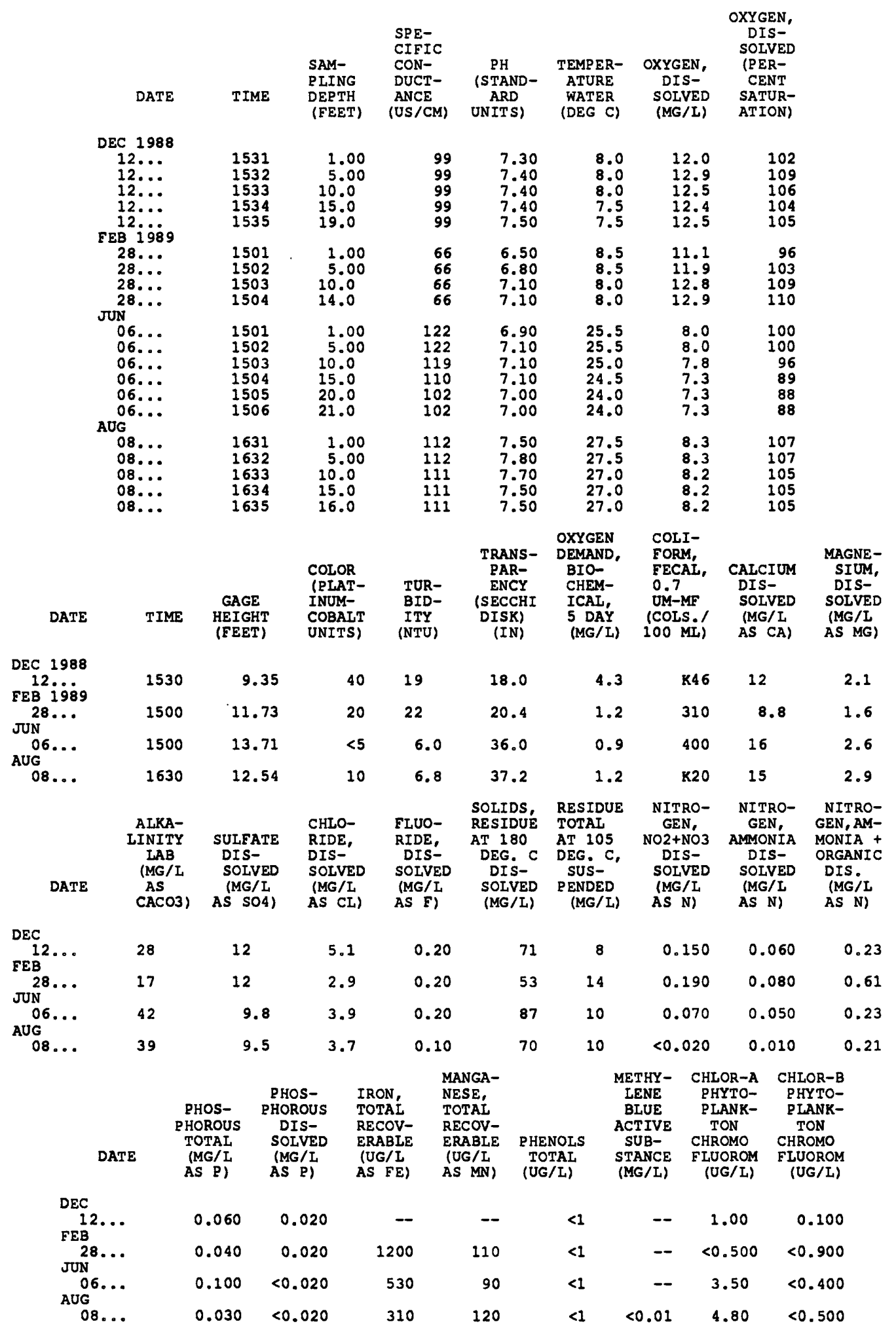


SURFACE-WATER SITES--Cont Inued

02430500 TOMBIGBEE RIVER NEAR MARIETTA, MS

\begin{tabular}{|c|c|c|c|c|c|c|c|c|c|}
\hline DATE & TIME & $\begin{array}{l}\text { DIS- } \\
\text { CHARGE, } \\
\text { INST. } \\
\text { CUBIC } \\
\text { FEET } \\
\text { PER } \\
\text { SECOND }\end{array}$ & $\begin{array}{l}\text { SEDI- } \\
\text { MENT, } \\
\text { SUS- } \\
\text { PENDED } \\
\text { (MG/L) }\end{array}$ & $\begin{array}{c}\text { SEDI- } \\
\text { MENT, } \\
\text { DIS- } \\
\text { CHARGE, } \\
\text { SUS- } \\
\text { PENDED } \\
\text { (T/DAY) }\end{array}$ & DATE & TIME & $\begin{array}{l}\text { DIS- } \\
\text { CHARGE, } \\
\text { INST. } \\
\text { CUBIC } \\
\text { FEET } \\
\text { PER } \\
\text { SECOND }\end{array}$ & $\begin{array}{l}\text { SEDI- } \\
\text { MENT, } \\
\text { SUS- } \\
\text { PENDED } \\
\text { (MG/L) }\end{array}$ & $\begin{array}{c}\text { SEDI- } \\
\text { MENT, } \\
\text { DIS- } \\
\text { CHARGE, } \\
\text { SUS- } \\
\text { PENDED } \\
\text { (T/DAY) }\end{array}$ \\
\hline $\begin{array}{l}\text { NOV } 1988 \\
17 \ldots \\
\text { DEC } \\
29 \ldots\end{array}$ & $\begin{array}{l}1120 \\
1400\end{array}$ & $\begin{array}{l}160 \\
773\end{array}$ & $\begin{array}{r}18 \\
217\end{array}$ & 7.8 & $\begin{array}{c}\text { MAY } 1989 \\
03 \ldots\end{array}$ & 1015 & 169 & 59 & 27 \\
\hline
\end{tabular}

02430680 TWENTYMILE CREEK NEAR GUNTOWN, MS

\begin{tabular}{|c|c|c|c|c|c|c|c|c|c|}
\hline DATE & TIME & $\begin{array}{c}\text { DIS- } \\
\text { CHARGE, } \\
\text { INST. } \\
\text { CUBIC } \\
\text { FEET } \\
\text { PER } \\
\text { SECOND }\end{array}$ & $\begin{array}{l}\text { SEDI- } \\
\text { MENT, } \\
\text { SUS- } \\
\text { PENDED } \\
\text { (MG/L) }\end{array}$ & $\begin{array}{c}\text { SEDI- } \\
\text { MENT, } \\
\text { DIS- } \\
\text { CHARGE, } \\
\text { SUS- } \\
\text { PENDED } \\
\text { (T/DAY) }\end{array}$ & DATE & TIME & $\begin{array}{c}\text { DIS- } \\
\text { CHARGE, } \\
\text { INST. } \\
\text { CUBIC } \\
\text { FEET } \\
\text { PER } \\
\text { SECOND }\end{array}$ & $\begin{array}{l}\text { SEDI- } \\
\text { MENT, } \\
\text { SUS- } \\
\text { PENDED } \\
\text { (MG/L) }\end{array}$ & $\begin{array}{c}\text { SEDI- } \\
\text { MENT, } \\
\text { DIS- } \\
\text { CHARGE } \\
\text { SUS- } \\
\text { PENDEI } \\
\text { (T/DAY) }\end{array}$ \\
\hline $\begin{array}{c}\text { DEC } 1988 \\
29 \ldots \\
\text { EEB } 1989 \\
15 \ldots\end{array}$ & 1030 & 268 & 193 & $\begin{array}{l}140 \\
654\end{array}$ & $\begin{array}{l}\text { MAY } \\
05 \ldots \\
\text { JUN } \\
16 \ldots\end{array}$ & $\begin{array}{l}1300 \\
1415\end{array}$ & $\begin{array}{r}1510 \\
499\end{array}$ & 1150 & $\begin{array}{r}4690 \\
155\end{array}$ \\
\hline
\end{tabular}

02430690 TWENTYMILE CREEK NEAR MANTACHIE, MS

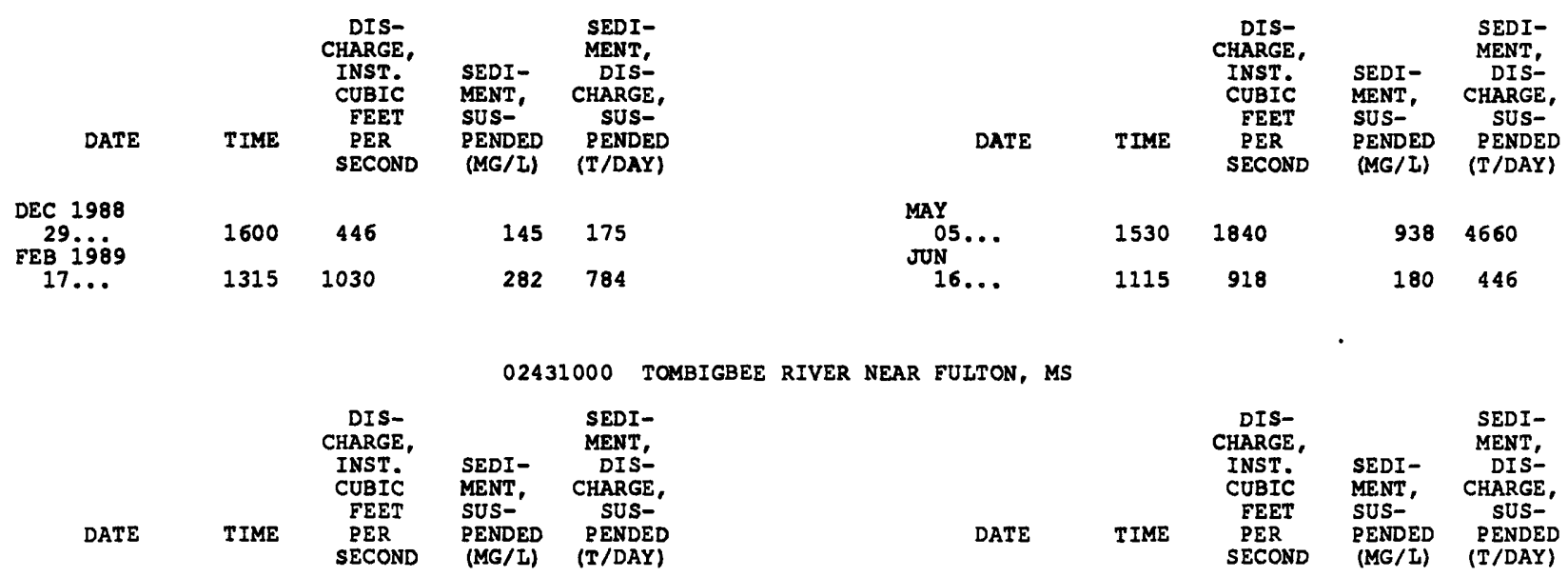

$\begin{array}{lrrrr}\begin{array}{l}\text { NOV } 1988 \\ 23 \ldots \ldots\end{array} & 1045 & 320 & 142 & 123 \\ \begin{array}{c}\text { DEC } \\ 28 \ldots\end{array} & 1200 & 1690 & 1310 & 5980 \\ \text { JAN } 1989 & & & & \\ 20 \ldots \ldots & 1030 & 1510 & 121 & 493 \\ \text { EEB } & & & & \\ 21 \ldots . & 1150 & 9680 & 770 & 20100 \\ 21 \ldots . & 1940 & 13100 & 713 & 25200\end{array}$

\begin{tabular}{|c|c|c|c|c|}
\hline \multicolumn{5}{|l|}{ FEB } \\
\hline $\begin{array}{l}22 \ldots \\
23 \ldots \\
27 \ldots\end{array}$ & $\begin{array}{l}0600 \\
1445 \\
1125\end{array}$ & $\begin{array}{r}13400 \\
7650 \\
1590\end{array}$ & $\begin{array}{l}347 \\
197 \\
267\end{array}$ & $\begin{array}{r}12600 \\
4070 \\
1150\end{array}$ \\
\hline MAR & & & & \\
\hline $22 \ldots$ & 1420 & 940 & 129 & 327 \\
\hline & 1200 & 755 & 140 & 285 \\
\hline UN & & & & \\
\hline 1 & 1100 & 1850 & 110 & 549 \\
\hline
\end{tabular}


SURFACE-WATER SITES--Cont Inued

02431410 MANTACHIE CREEK BELOW DORSEY, MS

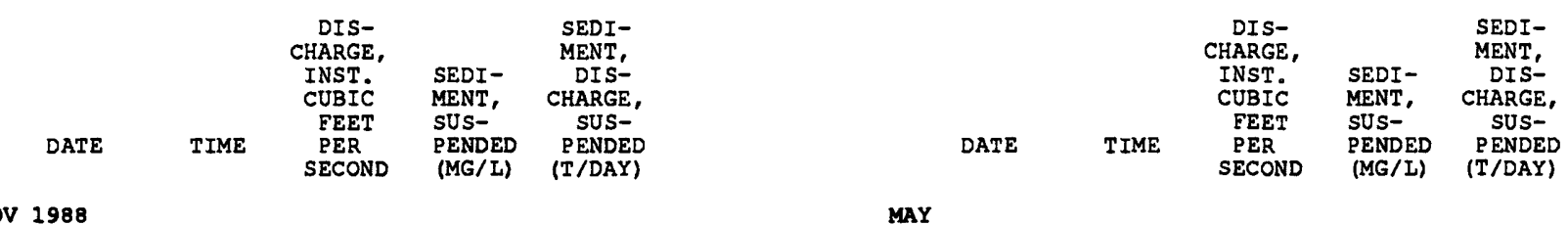

$\begin{array}{crcrc}\begin{array}{c}\text { NOV } 1988 \\ 22 \ldots . .\end{array} & 1520 & 6.6 & 66 & 1.2 \\ \text { DEC } & & & & \\ 28 \ldots & 1445 & 933 & 833 & 2100 \\ \text { FEB } 1989 & & & 909 & 1850 \\ 17 \ldots & 1100 & 752 & & \\ \text { MAR } & & & 2630 & 8880 \\ 05 \ldots . & 0100 & 1250 & 5290 & 38300 \\ 05 \ldots . & 0205 & 2680 & 3610 & 63200 \\ 05 \ldots . & 0515 & 6480 & 1650 & 30300 \\ 05 \ldots . & 0815 & 6810 & 1240 & 14800 \\ 05 \ldots & 1315 & 4410 & 1110 & 6410 \\ 05 \ldots & 1800 & 2140 & 73 & 7.7 \\ 22 \ldots & 1115 & 73 & 49 & 5.0 \\ 29 \ldots & 1405 & 42 & 44 & \end{array}$

$\begin{array}{rrr}\text { MAY } & & \\ 01 \ldots \ldots & 1400 & 102 \\ 09 \ldots & 1110 & 171 \\ 09 \ldots & 1840 & 145 \\ \pi N & & \\ 14 \ldots & 0215 & 103 \\ 14 \ldots . & 0510 & 159 \\ 14 \ldots & 0610 & 334 \\ 14 \ldots . & 0800 & 437 \\ 14 \ldots . & 1330 & 477 \\ 14 \ldots & 1545 & 518 \\ 15 \ldots \ldots & 0850 & 4170 \\ 15 \ldots \ldots & 1350 & 3250 \\ 16 \ldots \ldots & 0900 & 1210\end{array}$

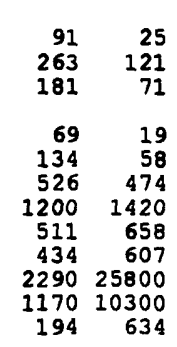

02433000 BULL MOUNTAIN CREEK NEAR SMITHVILLE, MS

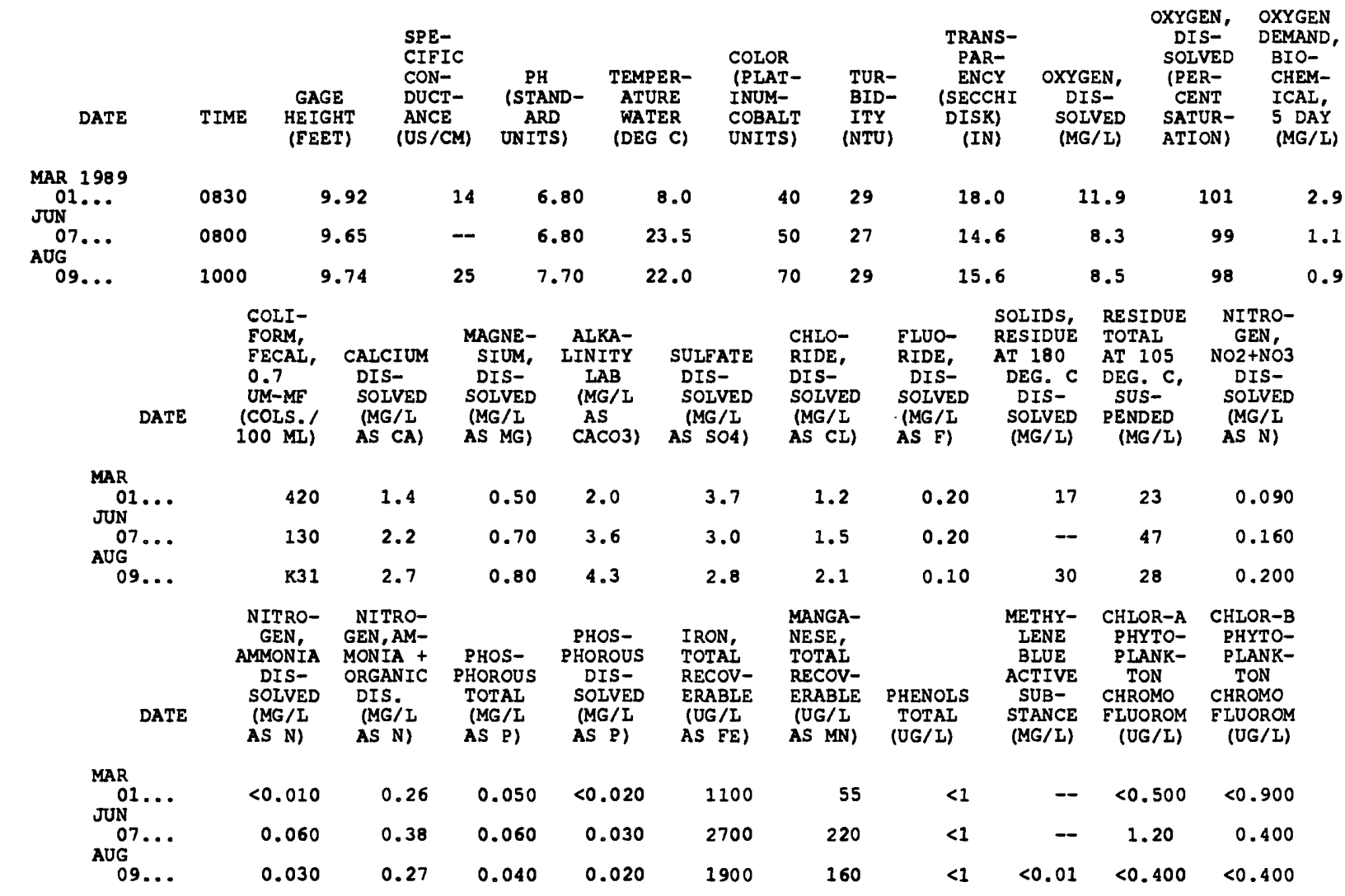

02433500 TOMBIGBEE RIVER AT BIGBEE, MS

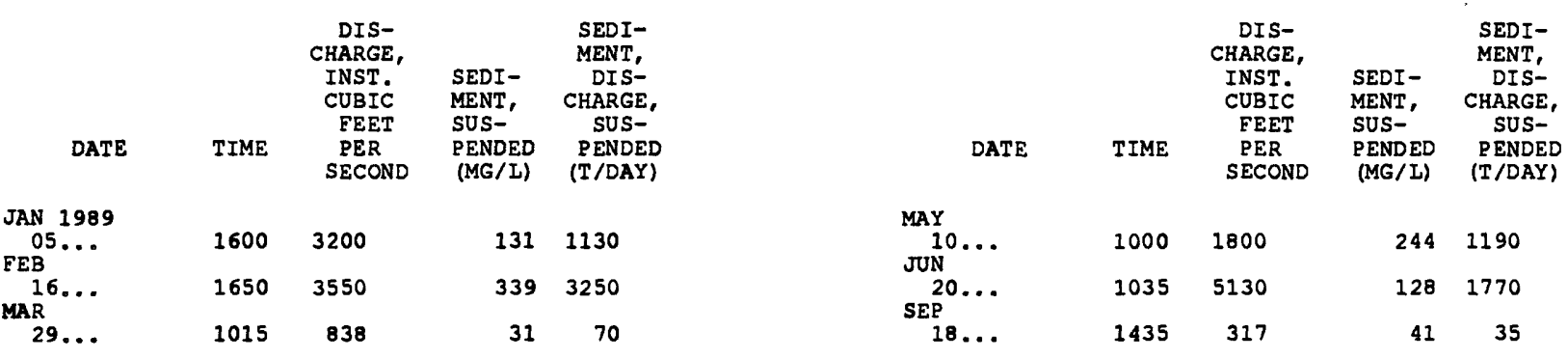


SURFACE-WATER SITES--Cont 1 nued

02436500 TOWN CREEK NEAR NETTIETON, MS

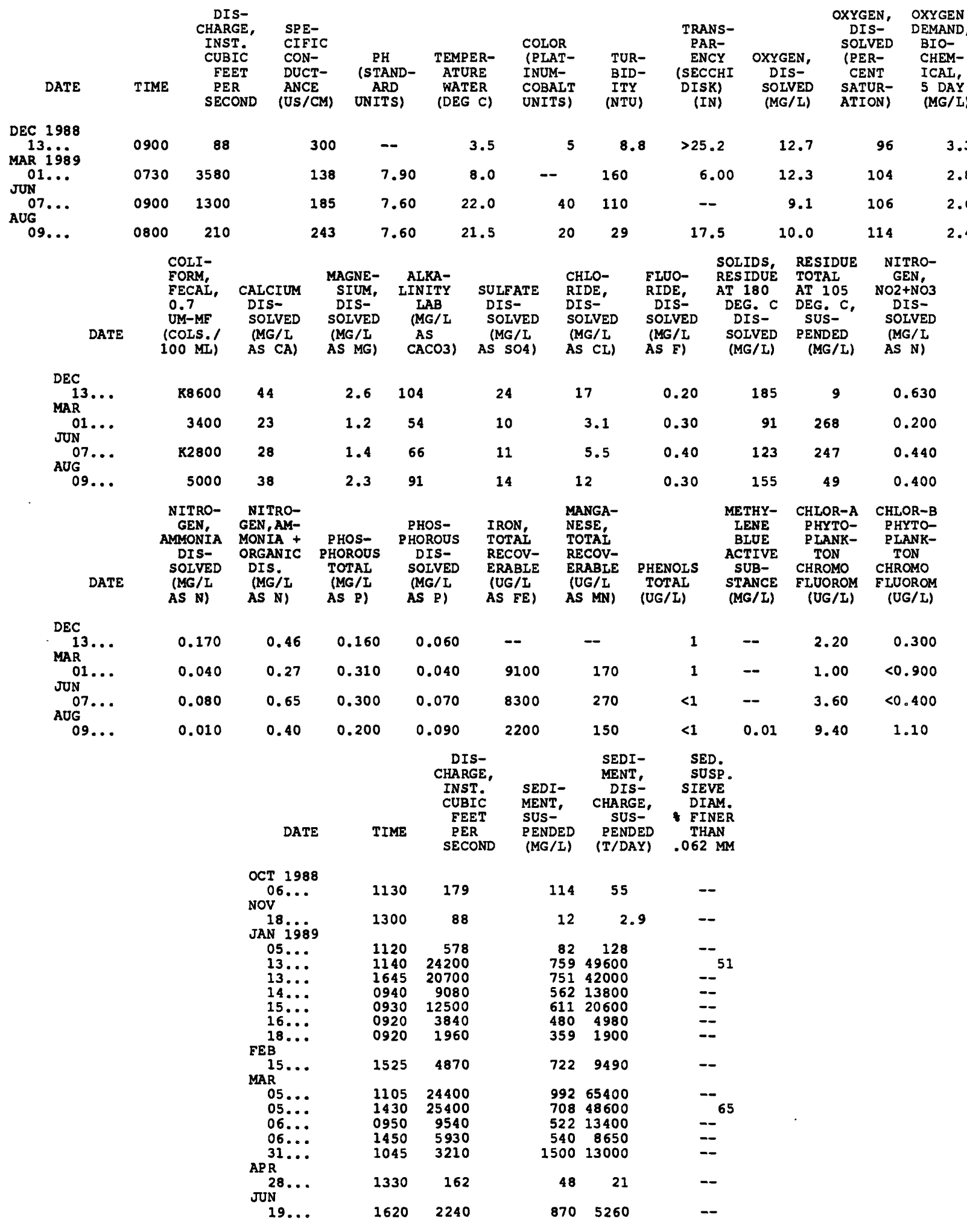


SURFACE-WATER SITES--Cont I nued

02436500 TOWN CREEK NEAR NETTLETON, MS--Continued

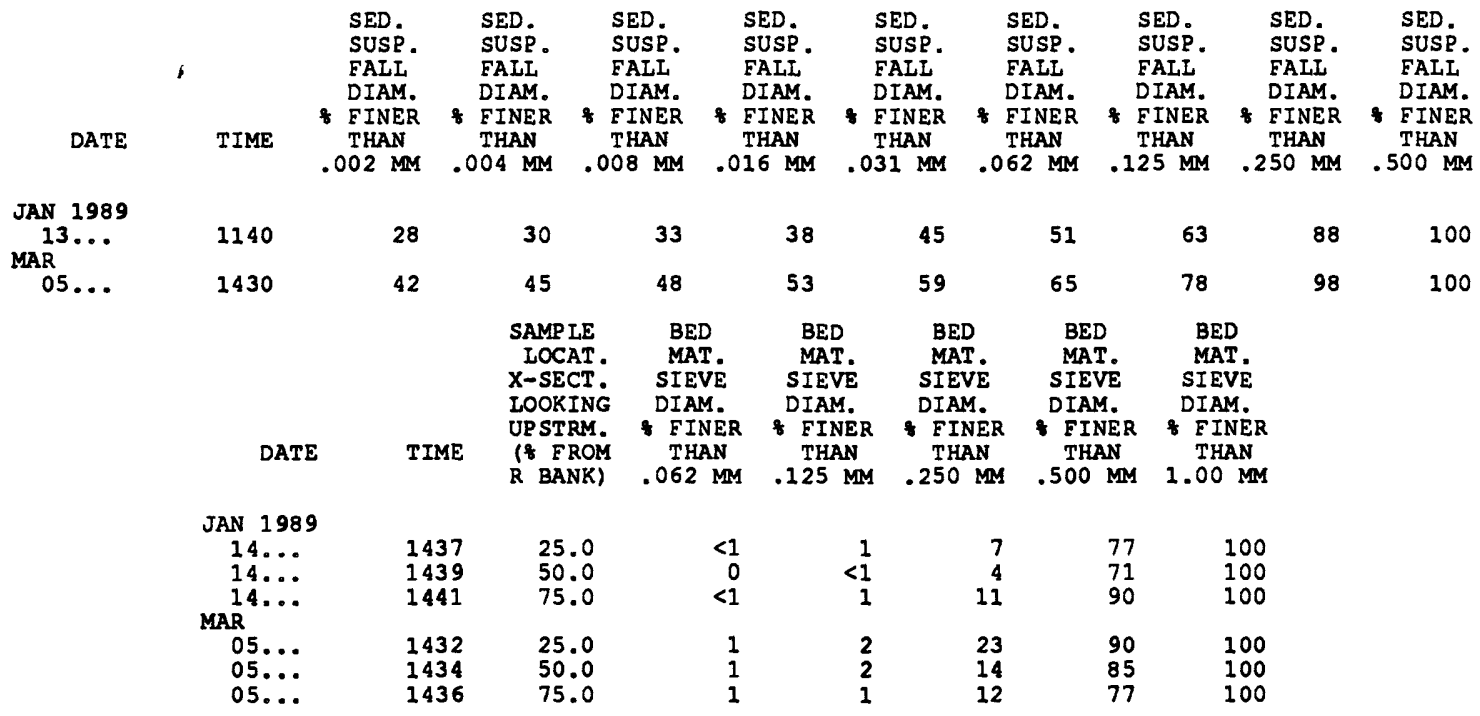

02437000 TOMBIGBEE RIVER NEAR AMORY, MS

\begin{tabular}{|c|c|c|c|c|}
\hline DATE & TIME & $\begin{array}{l}\text { DIS- } \\
\text { CHARGE, } \\
\text { INST. } \\
\text { CUBIC } \\
\text { FEET } \\
\text { PER } \\
\text { SECOND }\end{array}$ & $\begin{array}{l}\text { SEDI- } \\
\text { MENT, } \\
\text { SUS- } \\
\text { PENDED } \\
\text { (MG/L) }\end{array}$ & $\begin{array}{l}\text { SEDI- } \\
\text { MENT, } \\
\text { DIS- } \\
\text { CHARGE, } \\
\text { SUS- } \\
\text { PENDED } \\
\text { (T/DAY) }\end{array}$ \\
\hline $\begin{array}{l}1989 \\
\ldots\end{array}$ & 1400 & 6010 & 206 & 3340 \\
\hline
\end{tabular}

02437500 TOMBIGBEE RIVER AT ABERDEEN, MS

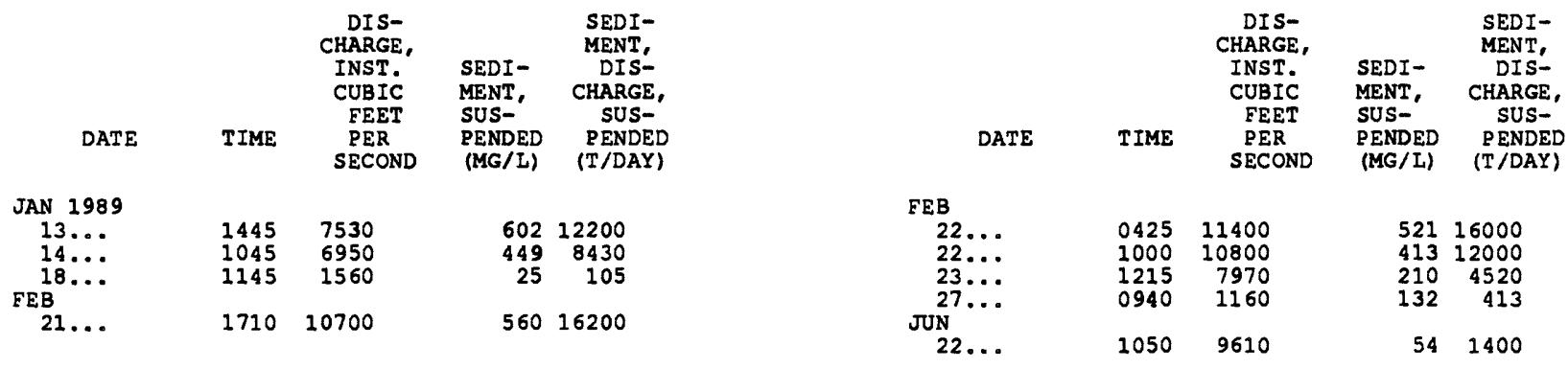


SUREACE-WATER SITES--Cont 1 nued

333927088304935 TTW COLUMBUS IAKE BUTTAHATCHEE RIVER BEND SR 26A

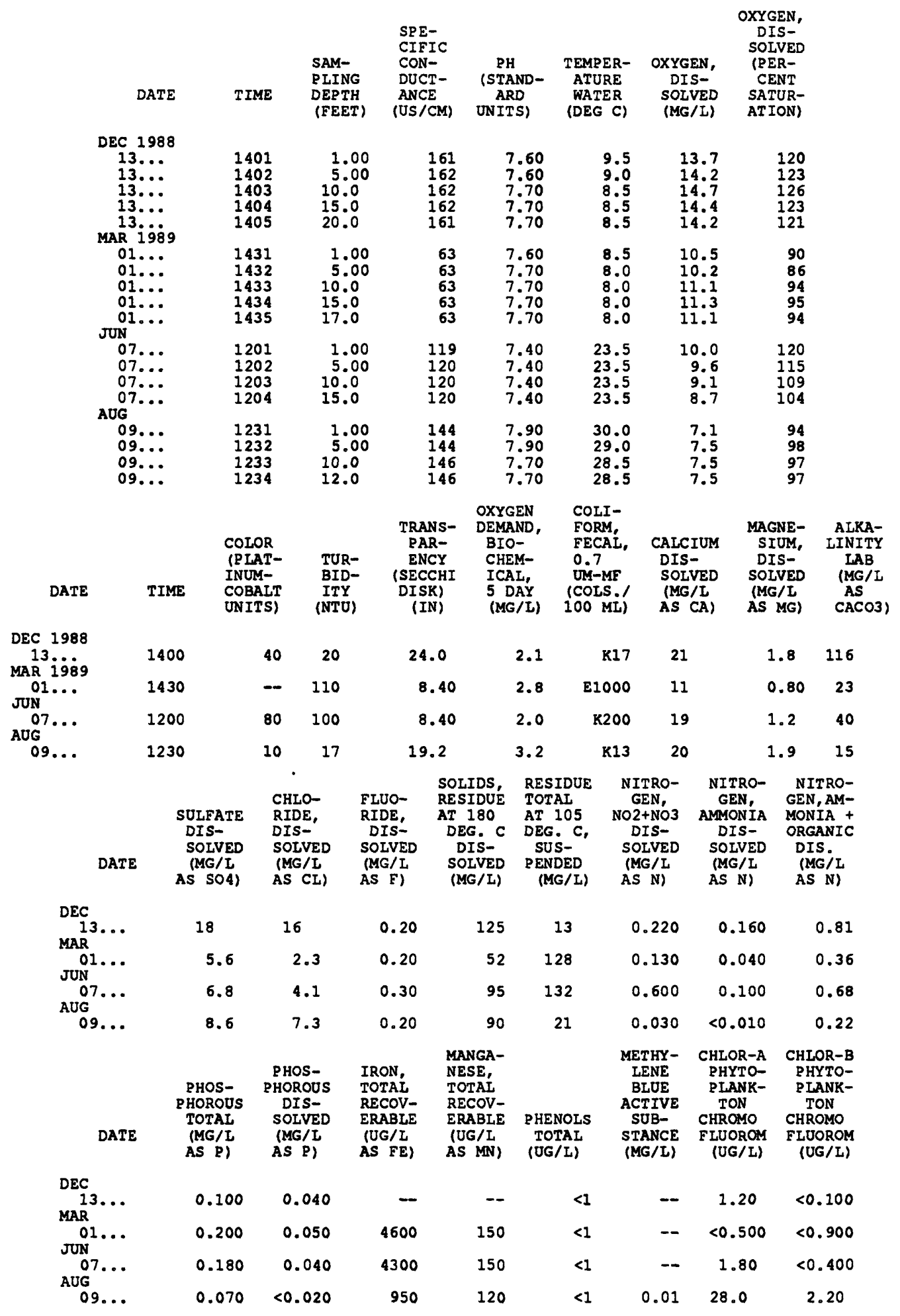


SURFACE-WATER SITES--Cont 1 nued

02439400 BUTTAHATCHEE RIVER NEAR ABERDEEN, MS

\begin{tabular}{|c|c|c|c|c|c|c|c|c|c|}
\hline DATE & TIME & $\begin{array}{c}\text { DIS- } \\
\text { CHARGE, } \\
\text { INST. } \\
\text { CUBIC } \\
\text { FEET } \\
\text { PER } \\
\text { SECOND }\end{array}$ & $\begin{array}{l}\text { SEDI- } \\
\text { MENT, } \\
\text { SUS- } \\
\text { PENDED } \\
\text { (MG/L) }\end{array}$ & $\begin{array}{c}\text { SEDI- } \\
\text { MENT, } \\
\text { DIS- } \\
\text { CHARGE, } \\
\text { SUS- } \\
\text { PENDED } \\
\text { (T/DAY) }\end{array}$ & DATE & TIME & $\begin{array}{c}\text { DIS- } \\
\text { CHARGE, } \\
\text { INST. } \\
\text { CUBIC } \\
\text { EEET } \\
\text { PER } \\
\text { SECOND }\end{array}$ & $\begin{array}{l}\text { SEDI- } \\
\text { MENT, } \\
\text { SUS- } \\
\text { PENDED } \\
\text { (MG/I) }\end{array}$ & $\begin{array}{r}\text { SEDI- } \\
\text { MENT, } \\
\text { DIS- } \\
\text { CHARGE, } \\
\text { SUS- } \\
\text { PENDED } \\
\text { (T/DAY) }\end{array}$ \\
\hline $\begin{array}{l}1988 \\
1989\end{array}$ & 1200 & 889 & 150 & 360 & $\begin{array}{l}\text { MAY } \\
10 \ldots \\
\text { JUN }\end{array}$ & 1430 & 1440 & 37 & 144 \\
\hline$\cdots$ & 1130 & 3030 & 40 & 327 & ${ }_{A U G}^{20} \cdots$ & 1645 & 2080 & 260 & 1460 \\
\hline$\cdots$ & 1230 & 1250 & 132 & 446 & $\begin{array}{c}01 \cdots \\
\text { SEP }\end{array}$ & 1515 & 594 & 32 & 51 \\
\hline . & 1000 & 1580 & 37 & 158 & $13 \ldots$ & 1330 & 561 & 59 & 89 \\
\hline
\end{tabular}

02439600 BUTTAHATCHEE RIVER NEAR KOLOLA SPRINGS, MS

\begin{tabular}{|c|c|c|c|c|c|c|c|c|c|c|c|}
\hline DATE & TIME & $\begin{array}{l}\text { DIS- } \\
\text { CHARGE, } \\
\text { INST. } \\
\text { CUBIC } \\
\text { FEET } \\
\text { PER } \\
\text { SECOND }\end{array}$ & $\begin{array}{l}\text { SPE- } \\
\text { CIFIC } \\
\text { CON- } \\
\text { DUCT- } \\
\text { ANCE } \\
\text { (US/CM) }\end{array}$ & $\begin{array}{c}\text { PH } \\
\text { (STAND- } \\
\text { ARD } \\
\text { UNITS) }\end{array}$ & $\begin{array}{c}\text { TEMPER- } \\
\text { ATURE } \\
\text { WATER } \\
\text { (DEG C) }\end{array}$ & $\begin{array}{l}\text { COLOR } \\
\text { (PLAT- } \\
\text { INUM- } \\
\text { COBALT } \\
\text { UNITS) }\end{array}$ & $\begin{array}{l}\text { TUR- } \\
\text { BID- } \\
\text { ITY } \\
\text { (NTU) }\end{array}$ & $\begin{array}{l}\text { TRANS- } \\
\text { PAR- } \\
\text { ENCY } \\
\text { (SECCHI } \\
\text { DISK) } \\
\text { (IN) }\end{array}$ & $\begin{array}{c}\text { OXYGEN, } \\
\text { DIS- } \\
\text { SOLVED } \\
\text { (MG/I) }\end{array}$ & $\begin{array}{c}\text { OXYGEN, } \\
\text { DIS- } \\
\text { SOLVED } \\
\text { (PER- } \\
\text { CENT } \\
\text { SATUR- } \\
\text { ATION) }\end{array}$ & $\begin{array}{l}\text { OXYGEN } \\
\text { DEMAND, } \\
\text { BIO- } \\
\text { CHEM- } \\
\text { ICAL, } \\
5 \text { DAY } \\
\text { (MG/L) }\end{array}$ \\
\hline
\end{tabular}

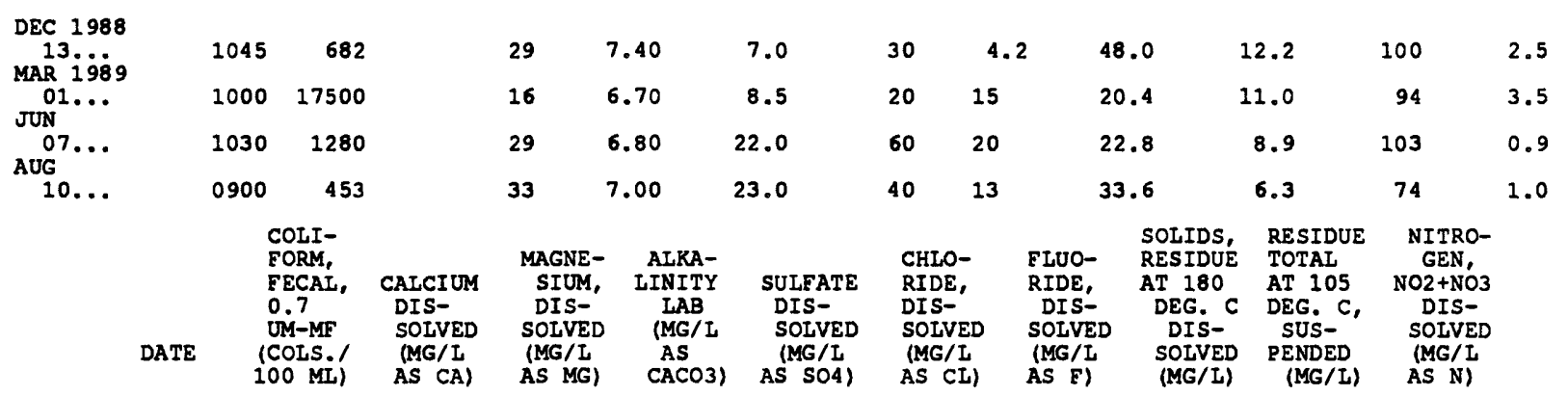

\begin{tabular}{|c|c|c|c|c|c|c|c|c|c|c|}
\hline $\begin{array}{c}\mathrm{DEC} \\
13 \ldots \\
\mathrm{MAR}\end{array}$ & K40 & 2.8 & 0.90 & 5.7 & 3.3 & 3.0 & 0.10 & 27 & 2 & 0.290 \\
\hline JUN $01 \ldots$ & K650 & 1.5 & 0.60 & 2.3 & 3.3 & 1.6 & 0.10 & 29 & 20 & 0.140 \\
\hline$\underset{A O G}{07 \ldots}$ & $\mathrm{K} 40$ & 2.5 & 0.80 & 5.6 & 2.8 & 2.2 & 0.30 & 39 & 43 & 0.220 \\
\hline $10 \ldots$ & K40 & 3.0 & 1.1 & 7.9 & 2.3 & 3.1 & 0.10 & 30 & 13 & 0.200 \\
\hline DATE & $\begin{array}{c}\text { NITRO- } \\
\text { GEN, } \\
\text { AMMONIA } \\
\text { DIS- } \\
\text { SOLVED } \\
\text { (MG/L } \\
\text { AS N) }\end{array}$ & $\begin{array}{l}\text { NITRO- } \\
\text { GEN, AM- } \\
\text { MONIA + } \\
\text { ORGANIC } \\
\text { DIS. } \\
\text { (MG/I } \\
\text { AS N) }\end{array}$ & $\begin{array}{l}\text { PHOS- } \\
\text { PHOROUS } \\
\text { TOTAL } \\
\text { (MG/I } \\
\text { AS P) }\end{array}$ & $\begin{array}{l}\text { PHOS- } \\
\text { PHOROUS } \\
\text { DIS- } \\
\text { SOLVED } \\
\text { (MG/I } \\
\text { AS P) }\end{array}$ & $\begin{array}{l}\text { IRON, } \\
\text { TOTAL } \\
\text { RECOV- } \\
\text { ERABLE } \\
\text { (UG/I } \\
\text { AS FE) }\end{array}$ & $\begin{array}{l}\text { MANGA- } \\
\text { NESE, } \\
\text { TOTAL } \\
\text { RECOV- } \\
\text { ERABLE } \\
\text { (UG/L } \\
\text { AS MN) }\end{array}$ & $\begin{array}{l}\text { PHENOLS } \\
\text { TOTAL } \\
\text { (UG/I) }\end{array}$ & $\begin{array}{l}\text { METHY- } \\
\text { LENE } \\
\text { BLUE } \\
\text { ACTIVE } \\
\text { SUB- } \\
\text { STANCE } \\
\text { (MG/L) }\end{array}$ & $\begin{array}{c}\text { CHLOR-A } \\
\text { PHYTO- } \\
\text { PLANK- } \\
\text { TON } \\
\text { CHROMO } \\
\text { FLUOROM } \\
\text { (UG/L) }\end{array}$ & $\begin{array}{c}\text { CHLOR-B } \\
\text { PHYTO- } \\
\text { PILANK- } \\
\text { TON } \\
\text { CHROMO } \\
\text { FLUOROM } \\
\text { (UG/L) }\end{array}$ \\
\hline
\end{tabular}

\begin{tabular}{|c|c|c|c|c|c|c|c|c|c|c|}
\hline $\begin{array}{l}\mathrm{DEC} \\
13 \ldots \\
\mathrm{MAR}\end{array}$ & 0.020 & $<0.20$ & 0.040 & 0.040 & - & -- & $<1$ & -- & $<0.500$ & $<0.100$ \\
\hline JUN & $<0.010$ & 0.24 & 0.040 & 0.030 & 1000 & 78 & $<1$ & - & 0.500 & 0.900 \\
\hline AUG $07 \ldots$ & 0.060 & 0.50 & 0.050 & $<0.020$ & 2900 & 250 & $<1$ & -- & 0.800 & $<0.400$ \\
\hline $10 \ldots$ & 0.030 & 0.39 & 0.020 & $<0.020$ & 1700 & 240 & $<1$ & $<0.01$ & 0.800 & $<0.400$ \\
\hline
\end{tabular}

02441000 TIBBEE CREEK NEAR TIBBEE, MS

\begin{tabular}{|c|c|c|c|c|c|c|c|c|c|c|c|}
\hline DATE & TIME & $\begin{array}{c}\text { DIS- } \\
\text { CHARGE, } \\
\text { INST. } \\
\text { CUBIC } \\
\text { FEET } \\
\text { PER } \\
\text { SECOND }\end{array}$ & $\begin{array}{l}\text { SPE- } \\
\text { CIFIC } \\
\text { CON- } \\
\text { DUCT- } \\
\text { ANCE } \\
\text { (US/CM) }\end{array}$ & $\begin{array}{c}\text { PH } \\
\text { (STAND- } \\
\text { ARD } \\
\text { UNITS) }\end{array}$ & $\begin{array}{c}\text { TEMPER- } \\
\text { ATURE } \\
\text { WATER } \\
\text { (DEG C) }\end{array}$ & $\begin{array}{l}\text { COLOR } \\
\text { (PLAT- } \\
\text { INUM- } \\
\text { COBALT } \\
\text { UNITS) }\end{array}$ & $\begin{array}{l}\text { TUR- } \\
\text { BID- } \\
\text { ITY } \\
\text { (NTU) }\end{array}$ & $\begin{array}{c}\text { TRANS- } \\
\text { PAR- } \\
\text { ENCY } \\
\text { (SECCHI } \\
\text { DISK) } \\
\text { (IN) }\end{array}$ & $\begin{array}{c}\text { OXYGEN, } \\
\text { DIS- } \\
\text { SOLVED } \\
\text { (MG/L) }\end{array}$ & $\begin{array}{c}\text { OXYGEN, } \\
\text { DIS- } \\
\text { SOLVED } \\
\text { (PER- } \\
\text { CENT } \\
\text { SATUR- } \\
\text { ATION) }\end{array}$ & $\begin{array}{c}\text { OXYGEN } \\
\text { DEMAND, } \\
\text { BIO- } \\
\text { CHEM- } \\
\text { ICAL, } \\
5 \mathrm{DAY} \\
\text { (MG/L) }\end{array}$ \\
\hline $\begin{array}{l}1988 \\
19899\end{array}$ & 1530 & $<700$ & 269 & 8.00 & 7.5 & 5 & 3.5 & 32.4 & 13.8 & 115 & 3.6 \\
\hline $1 \ldots$ & 1200 & 22100 & 65 & 7.60 & 8.5 & -- & 140 & 6.00 & 10.0 & 85 & 3.2 \\
\hline $7 \ldots$ & 1330 & 3300 & 140 & 7.20 & 22.5 & 60 & 22 & -- & 7.5 & 88 & 2.0 \\
\hline & 0730 & $<700$ & 188 & 7.50 & 22.5 & 30 & 33 & 12.0 & 7.1 & 82 & 1.6 \\
\hline
\end{tabular}


SURFACE-WATER SITES--Cont1 nued

02441000 TIBBEE CREEK NEAR TIBBEE, MS--COnt1nued

\begin{tabular}{|c|c|c|c|c|c|c|c|c|c|c|}
\hline DATE & $\begin{array}{l}\text { COLI- } \\
\text { FORM, } \\
\text { FECAL, } \\
0.7 \\
\text { UM-MF } \\
\text { (COLS.' } \\
\text { IOO ML) }\end{array}$ & $\begin{array}{l}\text { CALCIUM } \\
\text { DIS- } \\
\text { SOLVED } \\
\text { (MG/L } \\
\text { AS CA) }\end{array}$ & $\begin{array}{l}\text { MAGNE- } \\
\text { SIUM, } \\
\text { DIS- } \\
\text { SOIVED } \\
\text { (MG/I } \\
\text { AS MG) }\end{array}$ & $\begin{array}{c}\text { ALKA- } \\
\text { LINITY } \\
\text { LAB } \\
\text { (MG/L } \\
\text { AS } \\
\text { CACO3) }\end{array}$ & $\begin{array}{l}\text { SULFATE } \\
\text { DIS- } \\
\text { SOLVED } \\
\text { (MG/L } \\
\text { AS SO4) }\end{array}$ & $\begin{array}{l}\text { CHLO- } \\
\text { RIDE, } \\
\text { DIS- } \\
\text { SOIVED } \\
\text { (MG/I } \\
\text { AS CI) }\end{array}$ & $\begin{array}{l}\text { FLUO- } \\
\text { RIDE, } \\
\text { DIS- } \\
\text { SOIVED } \\
\text { (MG/I } \\
\text { AS F) }\end{array}$ & $\begin{array}{l}\text { SOLIDS, } \\
\text { RESIDUE } \\
\text { AT } 180 \\
\text { DEG. C } \\
\text { DIS- } \\
\text { SOIVED } \\
\text { (MG/L) }\end{array}$ & $\begin{array}{l}\text { RESIDUE } \\
\text { TOTAL } \\
\text { AT } 105 \\
\text { DEG. C, } \\
\text { SUS- } \\
\text { PENDED } \\
\text { (MG/L) }\end{array}$ & $\begin{array}{c}\text { NITRO- } \\
\text { GEN, } \\
\text { NO2+NO3 } \\
\text { DIS- } \\
\text { SOIVED } \\
\text { (MG/L } \\
\text { AS N) }\end{array}$ \\
\hline $\begin{array}{l}13 \ldots \\
13\end{array}$ & K37 & 45 & 2.6 & 107 & 22 & 11 & 0.20 & 171 & 2 & 0.070 \\
\hline UN & E2000 & 10 & 1.2 & 22 & 7.9 & 1.8 & 0.20 & 58 & 156 & 0.080 \\
\hline $07 \ldots$ & $\mathrm{K} 110$ & 21 & 1.5 & 42 & 10 & 5.2 & 0.30 & 121 & 44 & 0.940 \\
\hline $10 \ldots$ & $\mathrm{K} 170$ & 28 & 2.1 & 69 & 12 & 4.9 & 0.30 & 118 & 45 & 0.100 \\
\hline DATE & $\begin{array}{l}\text { NITRO- } \\
\text { GEN, } \\
\text { AMMONIA } \\
\text { DIS- } \\
\text { SOLVED } \\
\text { (MG/I } \\
\text { AS N) }\end{array}$ & $\begin{array}{l}\text { NITRO- } \\
\text { GEN,AY- } \\
\text { MONIA + } \\
\text { ORGANIC } \\
\text { DIS. } \\
\text { (MG/I } \\
\text { AS N) }\end{array}$ & $\begin{array}{l}\text { PHOS- } \\
\text { PHOROUS } \\
\text { TOTAL } \\
\text { (MG/L } \\
\text { AS P) }\end{array}$ & $\begin{array}{l}\text { PHOS- } \\
\text { PHOROUS } \\
\text { DIS- } \\
\text { SOLVED } \\
\text { (MG/I } \\
\text { AS P) }\end{array}$ & $\begin{array}{l}\text { IRON, } \\
\text { TOTAL } \\
\text { RECOV- } \\
\text { ERABLE } \\
\text { (UG/L } \\
\text { AS EE) }\end{array}$ & $\begin{array}{l}\text { MANGA- } \\
\text { NESE, } \\
\text { TOTAL } \\
\text { RECOV- } \\
\text { ERABLE } \\
\text { (UG/L } \\
\text { AS MN) }\end{array}$ & $\begin{array}{l}\text { PHENOLS } \\
\text { TOTAL } \\
\text { (UG/L) }\end{array}$ & $\begin{array}{l}\text { METHY- } \\
\text { LENE } \\
\text { BLUE } \\
\text { ACTIVE } \\
\text { SUB- } \\
\text { STANCE } \\
\text { (MG/L) }\end{array}$ & $\begin{array}{c}\text { CHLOR-A } \\
\text { RHYTO- } \\
\text { P IANK- } \\
\text { TON } \\
\text { CHROMO } \\
\text { FLUOROM } \\
\text { (UG/L) }\end{array}$ & $\begin{array}{c}\text { CHLOR-B } \\
\text { PHYTO- } \\
\text { PIANK- } \\
\text { TON } \\
\text { CHROMO } \\
\text { FLUOROM } \\
\text { (UG/I) }\end{array}$ \\
\hline $\begin{array}{l}\mathrm{EC} \\
13 \ldots \\
\mathrm{AR}\end{array}$ & 0.010 & 0.25 & 0.080 & 0.020 & -- & -- & $<1$ & -- & 2.00 & 0.200 \\
\hline UN $01 \ldots$ & $<0.010$ & $<0.20$ & 0.200 & 0.040 & 4500 & 150 & $<1$ & -- & $<0.500$ & $<0.900$ \\
\hline $\begin{array}{c}07 \ldots \\
\text { JG } \\
10 \ldots\end{array}$ & 0.050 & 0.55 & 0.170 & 0.080 & 1500 & 120 & $<1$ & $\begin{array}{r}-- \\
0.01\end{array}$ & 0.900 & $<0.400$ \\
\hline
\end{tabular}

02441400 TOMBIGBEE RIVER NEAR COLUMBUS, MS

\begin{tabular}{|c|c|c|c|c|c|c|c|c|c|}
\hline & DATE & TIME & $\begin{array}{l}\text { SAM- } \\
\text { PLING } \\
\text { DEPTH } \\
\text { (FEET) }\end{array}$ & $\begin{array}{l}\text { SPE- } \\
\text { CIFIC } \\
\text { CON- } \\
\text { DUCT- } \\
\text { ANCE } \\
\text { (US/CM) }\end{array}$ & $\begin{array}{c}\text { PH } \\
\text { (STAND- } \\
\text { ARD } \\
\text { UNITS) }\end{array}$ & $\begin{array}{c}\text { TEMPER- } \\
\text { ATURE } \\
\text { WATER } \\
\text { (DEG C) }\end{array}$ & $\begin{array}{c}\text { OXYGEN, } \\
\text { DIS- } \\
\text { SOIVED } \\
\text { (MG/L) }\end{array}$ & $\begin{array}{c}\text { OXYGEN, } \\
\text { DIS- } \\
\text { SOLVED } \\
\text { (PER- } \\
\text { CENT } \\
\text { SATUR- } \\
\text { ATION) }\end{array}$ & \\
\hline & $\begin{array}{c}\text { DEC } 1988 \\
14 \ldots \\
14 \ldots \\
14 \ldots \\
14 \ldots \\
14 \ldots \\
\text { MAR } 1989\end{array}$ & $\begin{array}{l}0931 \\
0932 \\
0933 \\
0934 \\
0935\end{array}$ & $\begin{array}{c}1.00 \\
5.00 \\
10.0 \\
15.0 \\
20.0\end{array}$ & $\begin{array}{l}100 \\
100 \\
100 \\
100 \\
100\end{array}$ & $\begin{array}{l}7.30 \\
7.30 \\
7.40 \\
7.50 \\
7.50\end{array}$ & $\begin{array}{l}7.5 \\
7.5 \\
7.5 \\
7.5 \\
7.5\end{array}$ & $\begin{array}{l}12.5 \\
12.5 \\
12.0 \\
12.0 \\
12.0\end{array}$ & $\begin{array}{l}105 \\
105 \\
100 \\
100 \\
100\end{array}$ & \\
\hline & $\begin{array}{l}01 \ldots \\
01 \ldots \\
01 \ldots \\
01 \ldots \\
01 \ldots \\
01 \ldots \\
01 \ldots \\
\text { JUN }\end{array}$ & $\begin{array}{l}1601 \\
1602 \\
1603 \\
1604 \\
1605 \\
1606 \\
1607\end{array}$ & $\begin{array}{l}1.00 \\
5.00 \\
10.0 \\
15.0 \\
20.0 \\
25.0 \\
30.0\end{array}$ & $\begin{array}{l}74 \\
73 \\
72 \\
70 \\
71 \\
69 \\
68\end{array}$ & $\begin{array}{l}7.50 \\
7.60 \\
7.60 \\
7.70 \\
7.80 \\
7.80 \\
7.80\end{array}$ & $\begin{array}{l}8.0 \\
8.0 \\
8.0 \\
8.0 \\
8.0 \\
8.0 \\
8.0\end{array}$ & $\begin{array}{l}10.8 \\
10.7 \\
11.3 \\
11.4 \\
10.8 \\
11.0 \\
11.1\end{array}$ & $\begin{array}{l}91 \\
90 \\
95 \\
96 \\
91 \\
93 \\
94\end{array}$ & \\
\hline & $\begin{array}{r}07 \ldots \\
07 \ldots \\
07 \ldots \\
07 \ldots \\
07 \ldots \\
\text { AUG }\end{array}$ & $\begin{array}{l}1431 \\
1432 \\
1433 \\
1434 \\
1435\end{array}$ & $\begin{array}{l}1.00 \\
5.00 \\
10.0 \\
15.0 \\
18.0\end{array}$ & $\begin{array}{l}154 \\
148 \\
148 \\
144 \\
142\end{array}$ & $\begin{array}{l}7.40 \\
7.40 \\
7.40 \\
7.40 \\
7.30\end{array}$ & $\begin{array}{l}24.5 \\
24.5 \\
24.5 \\
24.5 \\
24.5\end{array}$ & $\begin{array}{r}10.2 \\
9.8 \\
9.2 \\
9.3 \\
9.5\end{array}$ & $\begin{array}{l}124 \\
119 \\
112 \\
113 \\
115\end{array}$ & \\
\hline & $\begin{array}{l}09 . \ldots \\
09 . \ldots \\
09 \ldots \\
09 \ldots\end{array}$ & $\begin{array}{l}1401 \\
1402 \\
1403 \\
1404\end{array}$ & $\begin{array}{r}1.00 \\
5.00 \\
10.0 \\
12.0\end{array}$ & $\begin{array}{l}165 \\
165 \\
166 \\
166\end{array}$ & $\begin{array}{l}-- \\
-- \\
--\end{array}$ & $\begin{array}{l}29.5 \\
28.5 \\
28.0 \\
28.0\end{array}$ & $\begin{array}{l}7.1 \\
6.0 \\
6.6 \\
6.6\end{array}$ & $\begin{array}{l}94 \\
78 \\
85 \\
85\end{array}$ & \\
\hline DATE & TIME & $\begin{array}{c}\text { DIS- } \\
\text { CHARGE, } \\
\text { INST. } \\
\text { CUBIC } \\
\text { FEET } \\
\text { PER } \\
\text { SECOND }\end{array}$ & $\begin{array}{l}\text { COLOR } \\
\text { (PIAT- } \\
\text { INUM- } \\
\text { COBALT } \\
\text { UNITS) }\end{array}$ & $\begin{array}{l}\text { TUR- } \\
\text { BID- } \\
\text { ITY } \\
\text { (NTU) }\end{array}$ & $\begin{array}{c}\text { TRANS- } \\
\text { PAR- } \\
\text { ENCY } \\
\text { (SECCHI } \\
\text { DISK) } \\
\text { (IN) }\end{array}$ & $\begin{array}{l}\text { OXYGEN } \\
\text { DEMAND, } \\
\text { BIO- } \\
\text { CHEM- } \\
\text { ICAL, } \\
5 \text { DAY } \\
\text { (MG/L) }\end{array}$ & $\begin{array}{l}\text { COLI- } \\
\text { FORM, } \\
\text { FECAL, } \\
0.7 \\
\text { UM-MF } \\
\text { (COLS.' } \\
100 \mathrm{ML} \text { ) }\end{array}$ & $\begin{array}{l}\text { CALCIUM } \\
\text { DIS- } \\
\text { SOLVED } \\
\text { (MG/L } \\
\text { AS CA) }\end{array}$ & $\begin{array}{l}\text { MAGNE- } \\
\text { SIUM, } \\
\text { DIS- } \\
\text { SOLVED } \\
\text { (MG/L } \\
\text { AS MG) }\end{array}$ \\
\hline $\begin{array}{c}\text { DEC } 1988 \\
14 . \ddot{8} \\
\text { MAR } 1989\end{array}$ & 0930 & 1560 & 40 & 16 & 19.2 & 1.9 & K22 & 12 & 1.4 \\
\hline JUN & 1600 & 48100 & -- & 130 & 8.40 & 3.3 & E3000 & 12 & 0.80 \\
\hline AUG & 1430 & 12900 & 40 & 53 & 8.40 & 2.0 & K300 & 21 & 1.3 \\
\hline $09 .$. & 1400 & 2350 & 10 & 17 & 19.2 & 3.8 & $<10$ & 24 & 1.7 \\
\hline
\end{tabular}


SURFACE-WATER SITES--Cont 1 nued

02441400 TOMBIGBEE RIVER NEAR COLUMBUS, MS--Continued

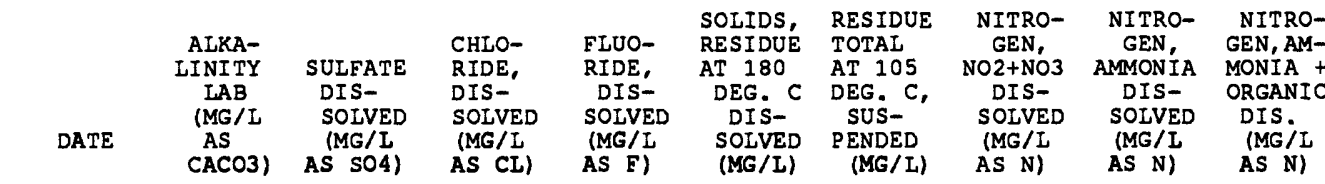

\begin{tabular}{|c|c|c|c|c|c|c|c|c|c|}
\hline $\begin{array}{l}\text { DEC } \\
14 \ldots\end{array}$ & 25 & 9.1 & 8.4 & 0.10 & 68 & 16 & 0.220 & 0.100 & 0.46 \\
\hline $\begin{array}{l}\text { MUR } \\
01 \ldots N \\
\text { JUN }\end{array}$ & 26 & 6.1 & 2.5 & 0.20 & 58 & 160 & 0.140 & 0.030 & 0.30 \\
\hline $\begin{array}{c}07 . . \\
\text { AUG }\end{array}$ & 46 & 8.5 & 6.5 & 0.30 & 110 & 78 & 0.770 & 0.100 & 0.61 \\
\hline $09 \ldots$ & 51 & 9.7 & 12 & 0.20 & 109 & 28 & $<0.020$ & $<0.010$ & 0.22 \\
\hline
\end{tabular}

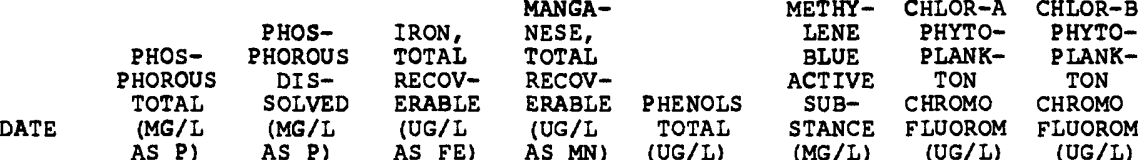

\begin{tabular}{|c|c|c|c|c|c|c|c|c|}
\hline $\begin{array}{l}\mathrm{DEC} \\
14 \ldots \\
\mathrm{MAR}\end{array}$ & 0.070 & 0.020 & - & -- & 1 & -- & 2.50 & 0.200 \\
\hline JUN & 0.250 & 0.030 & 4400 & 170 & $<1$ & - & $<0.500$ & $<0.900$ \\
\hline $\begin{array}{c}07 \ldots \\
\text { AUG }\end{array}$ & 0.150 & 0.030 & 2800 & 130 & $<1$ & & 3.60 & $<0.400$ \\
\hline $09 \ldots$ & 0.100 & $<0.020$ & 1000 & 120 & $<1$ & 0.01 & 22.0 & 1.90 \\
\hline
\end{tabular}

\begin{tabular}{|c|c|c|c|c|}
\hline DATE & TIME & $\begin{array}{l}\text { DIS- } \\
\text { CHARGE, } \\
\text { INST. } \\
\text { CUBIC } \\
\text { FEET } \\
\text { PER } \\
\text { SECOND }\end{array}$ & $\begin{array}{l}\text { SEDI- } \\
\text { MENT, } \\
\text { SUS- } \\
\text { PENDED } \\
\text { (MG/L) }\end{array}$ & $\begin{array}{c}\text { SEDI- } \\
\text { MENT, } \\
\text { DIS- } \\
\text { CHARGE, } \\
\text { SUS- } \\
\text { PENDED } \\
\text { (T/DAY) }\end{array}$ \\
\hline & & & & \\
\hline
\end{tabular}

$\begin{array}{ccccc}\text { JUN } 1989 & 1815 & 14900 & 106 & 4260\end{array}$

332929088273300 TTW AIICEVILIE LAKE ABOVE COLUMBUS BEND

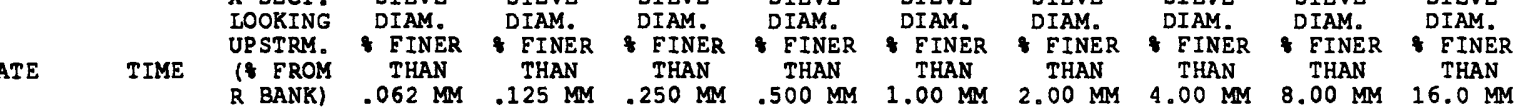

JUN

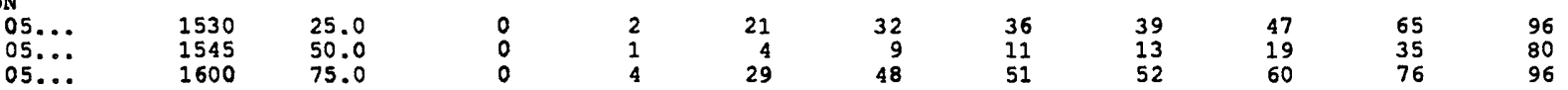


SURFACE-WATER SITES--Continued

02441498 TOMBIGBEE RIVER COLUMBUS BEND SR 11B AT COLUMBUS, MS

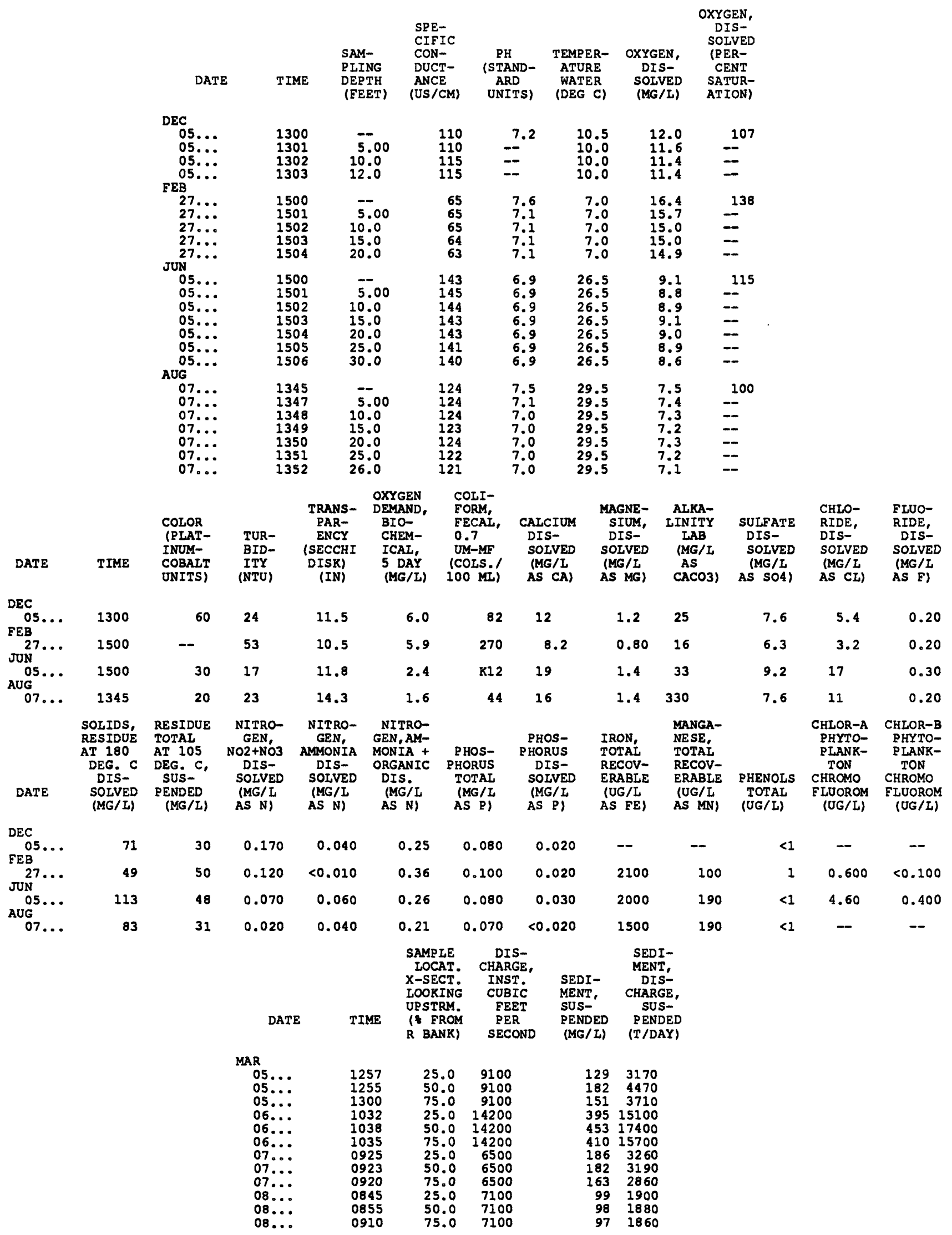


SURFACE-WATER SITES--Cont1 nued

02441498 TOMBIGBEE RIVER COLUMBUS BEND SR 11B AT COLUMBUS, MS--Cont1nued

\begin{tabular}{|c|c|c|c|c|c|c|c|c|c|c|c|}
\hline DATE & TIME & $\begin{array}{l}\text { SAMP LE } \\
\text { LOCAT. } \\
\text { X-SECT. } \\
\text { LOOKING } \\
\text { UP STRM. } \\
\text { (\& FROM } \\
\text { R BANK) }\end{array}$ & $\begin{array}{c}\text { BED } \\
\text { MAT. } \\
\text { SIEVE } \\
\text { DIAM. } \\
\text { FINER } \\
\text { THAN } \\
.062 \mathrm{MM}\end{array}$ & $\begin{array}{c}\text { BED } \\
\text { MAT. } \\
\text { SIEVE } \\
\text { DIAM. } \\
\text { FINER } \\
\text { THAN } \\
.125 \mathrm{MM}\end{array}$ & $\begin{array}{c}\text { BED } \\
\text { MAT. } \\
\text { SIEVE } \\
\text { DIAM. } \\
\text { \& FINER } \\
\text { THAN } \\
.250 \mathrm{MM}\end{array}$ & $\begin{array}{c}\text { BED } \\
\text { MAT. } \\
\text { SIEVE } \\
\text { DIAM. } \\
\text { FINER } \\
\text { THAN } \\
.500 \mathrm{MM}\end{array}$ & $\begin{array}{c}\text { BED } \\
\text { MAT. } \\
\text { SIEVE } \\
\text { DIAM. } \\
\text { \& FINER } \\
\text { THAN } \\
1.00 \mathrm{MM}\end{array}$ & $\begin{array}{c}\text { BED } \\
\text { MAT. } \\
\text { SIEVE } \\
\text { DIAM. } \\
\text { FINER } \\
\text { THAN } \\
2.00 \mathrm{MM}\end{array}$ & $\begin{array}{c}\text { BED } \\
\text { MAT. } \\
\text { SIEVE } \\
\text { DIAM. } \\
\text { \& EINER } \\
\text { THAN } \\
\text { 4.00 MM }\end{array}$ & $\begin{array}{c}\text { BED } \\
\text { MAT. } \\
\text { SIEVE } \\
\text { DIAM. } \\
\text { FINER } \\
\text { THAN } \\
8.00 \mathrm{MM}\end{array}$ & $\begin{array}{c}\text { BED } \\
\text { MAT. } \\
\text { SIEVE } \\
\text { DIAM. } \\
\text { FINER } \\
\text { THAN } \\
16.0 \mathrm{MM}\end{array}$ \\
\hline $\mathrm{N}$ & & & & & & & & & & & \\
\hline $\begin{array}{l}05 \ldots \\
05 \ldots \\
05 \ldots\end{array}$ & $\begin{array}{l}1745 \\
1800 \\
1815\end{array}$ & $\begin{array}{l}25.0 \\
50.0 \\
75.0\end{array}$ & $\begin{array}{r}1 \\
0 \\
15\end{array}$ & $\begin{array}{r}7 \\
2 \\
29\end{array}$ & $\begin{array}{r}19 \\
5 \\
33\end{array}$ & $\begin{array}{l}68 \\
10 \\
36\end{array}$ & $\begin{array}{l}76 \\
12 \\
39\end{array}$ & $\begin{array}{l}79 \\
13 \\
45\end{array}$ & $\begin{array}{l}81 \\
20 \\
56\end{array}$ & $\begin{array}{l}86 \\
38 \\
74\end{array}$ & \\
\hline
\end{tabular}

332751088261000 TTW ALICEVILLE LAKE COLUMBUS CUT

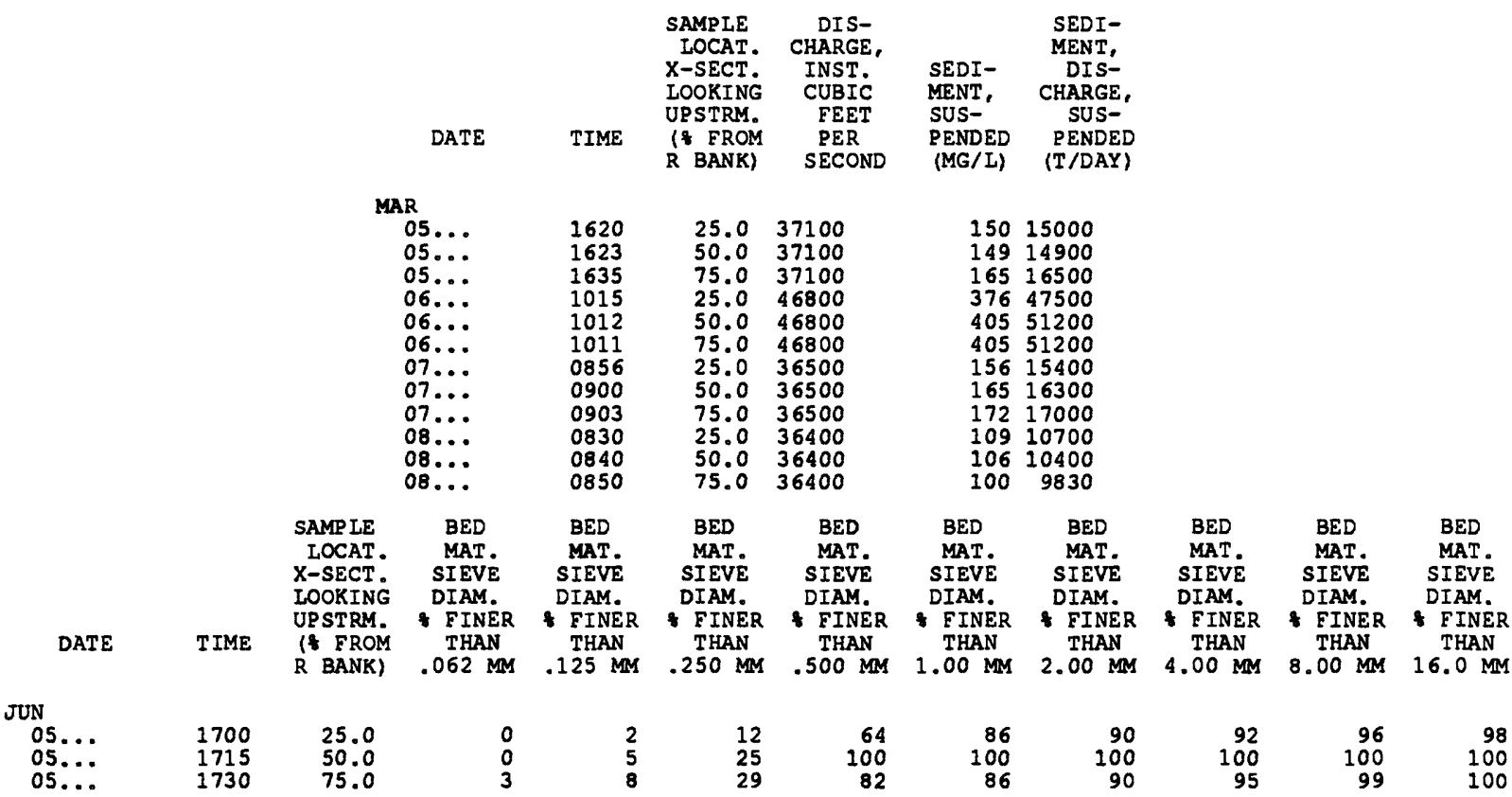

02443500 LUXAPALLILA CREEK NEAR COLUMBUS, MS

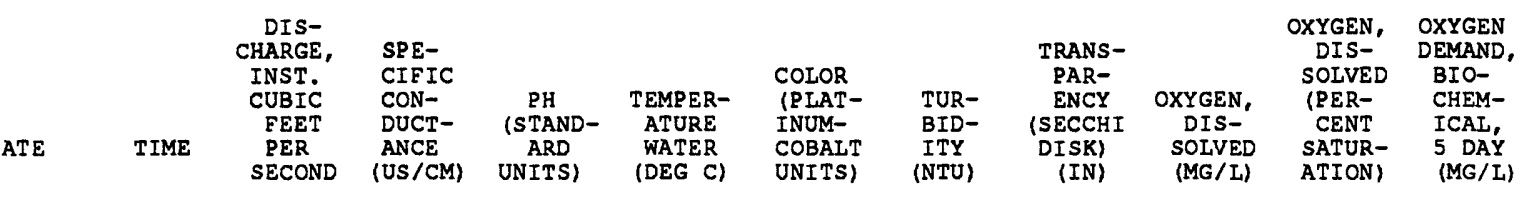

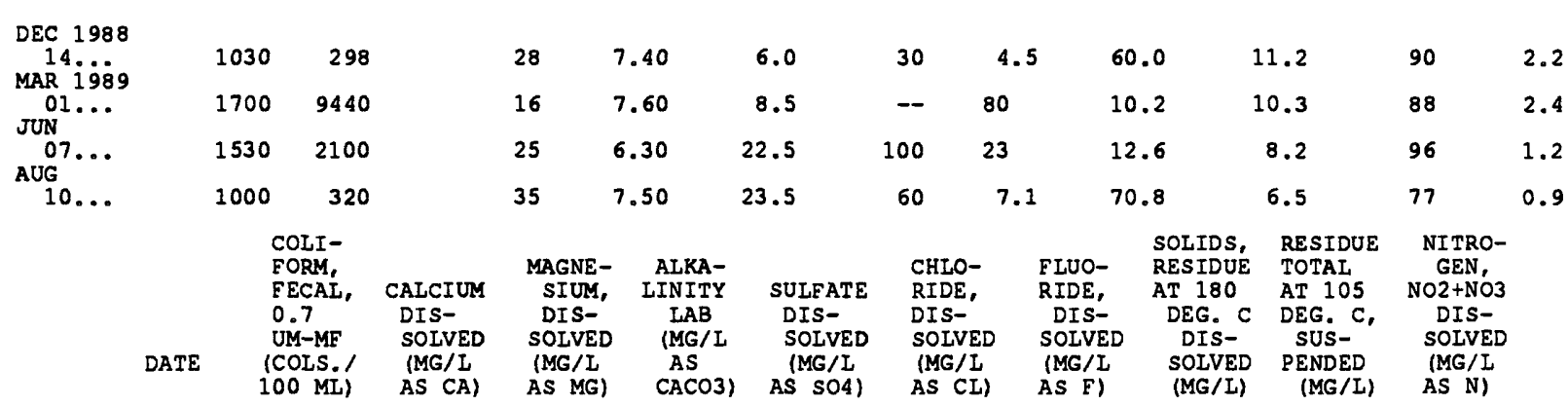

\begin{tabular}{|c|c|c|c|c|c|c|c|c|c|c|}
\hline $\begin{array}{l}\mathrm{DEC} \\
14 \ldots\end{array}$ & 48 & 2.1 & 0.80 & 3.6 & 4.2 & 3.1 & 0.10 & 28 & 12 & 0.150 \\
\hline $\begin{array}{c}\text { MAR } \\
01 \ldots \\
\text { JUN }\end{array}$ & E1200 & 1.5 & 0.50 & 1.6 & 3.5 & 1.4 & 0.20 & 29 & 46 & 0.080 \\
\hline${ }_{\text {AUG }}^{07 \ldots}$ & 200 & 2.5 & 0.70 & 2.5 & 3.4 & 1.8 & 0.20 & 43 & 36 & 0.620 \\
\hline $10 \ldots$ & K30 & 3.1 & 1.3 & 17 & 2.8 & 2.8 & $<0.10$ & 35 & 6 & 0.150 \\
\hline
\end{tabular}


02443500 LUXAPALLILA CREEK NEAR COLUMBUS, MS--Cont inued

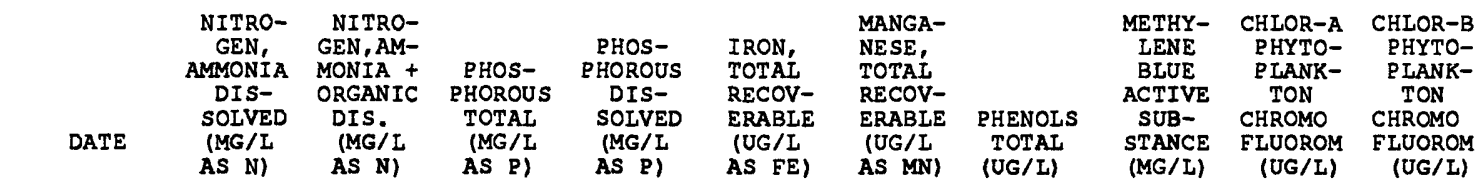

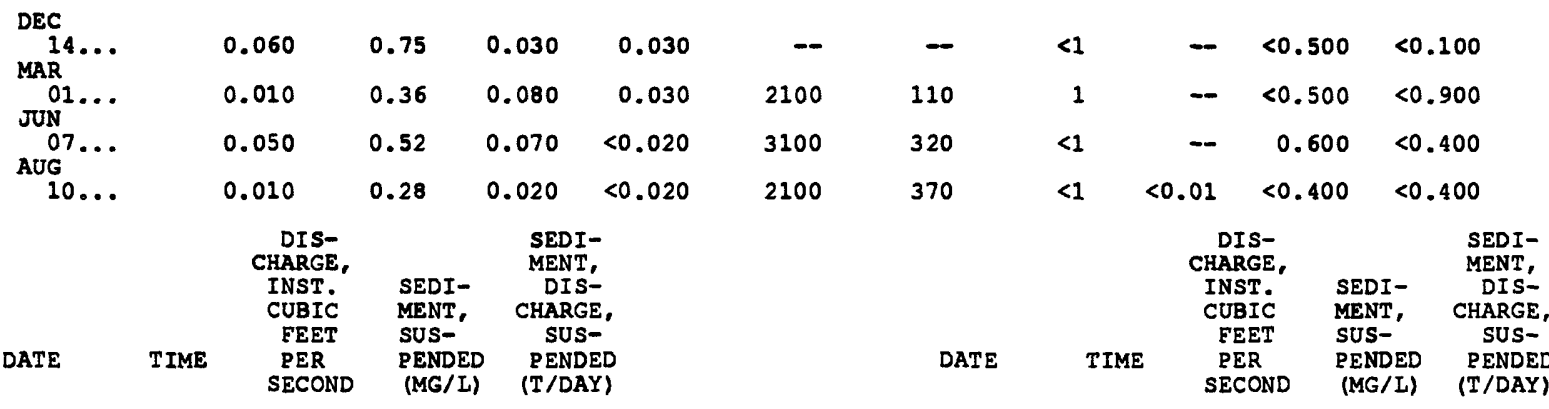

\begin{tabular}{|c|c|c|c|c|}
\hline $\begin{array}{l}\text { NOV } 1988 \\
15 \\
\text { JAN } 19899\end{array}$ & 1345 & 614 & 17 & 28 \\
\hline $\begin{array}{l}04 \ldots \\
\text { FEB }\end{array}$ & 1330 & 2960 & 12 & 96 \\
\hline & 1225 & 1040 & 73 & 205 \\
\hline $28 \ldots$ & 1000 & 1020 & 17 & 47 \\
\hline
\end{tabular}

$\begin{array}{lrrrr}\text { MAY } & & & \\ \text { 11... } & 0925 & 1270 & 23 & 79 \\ \text { JUN } & 1305 & 2640 & 27 & 192 \\ \text { AUG } & 120 & 538 & 18 & 26 \\ \text { O2... } & 1200 & 19 & 7.6\end{array}$

3321120882235 TTW ALICEVILLE LAKE ABOVE HAIRSTON BEND

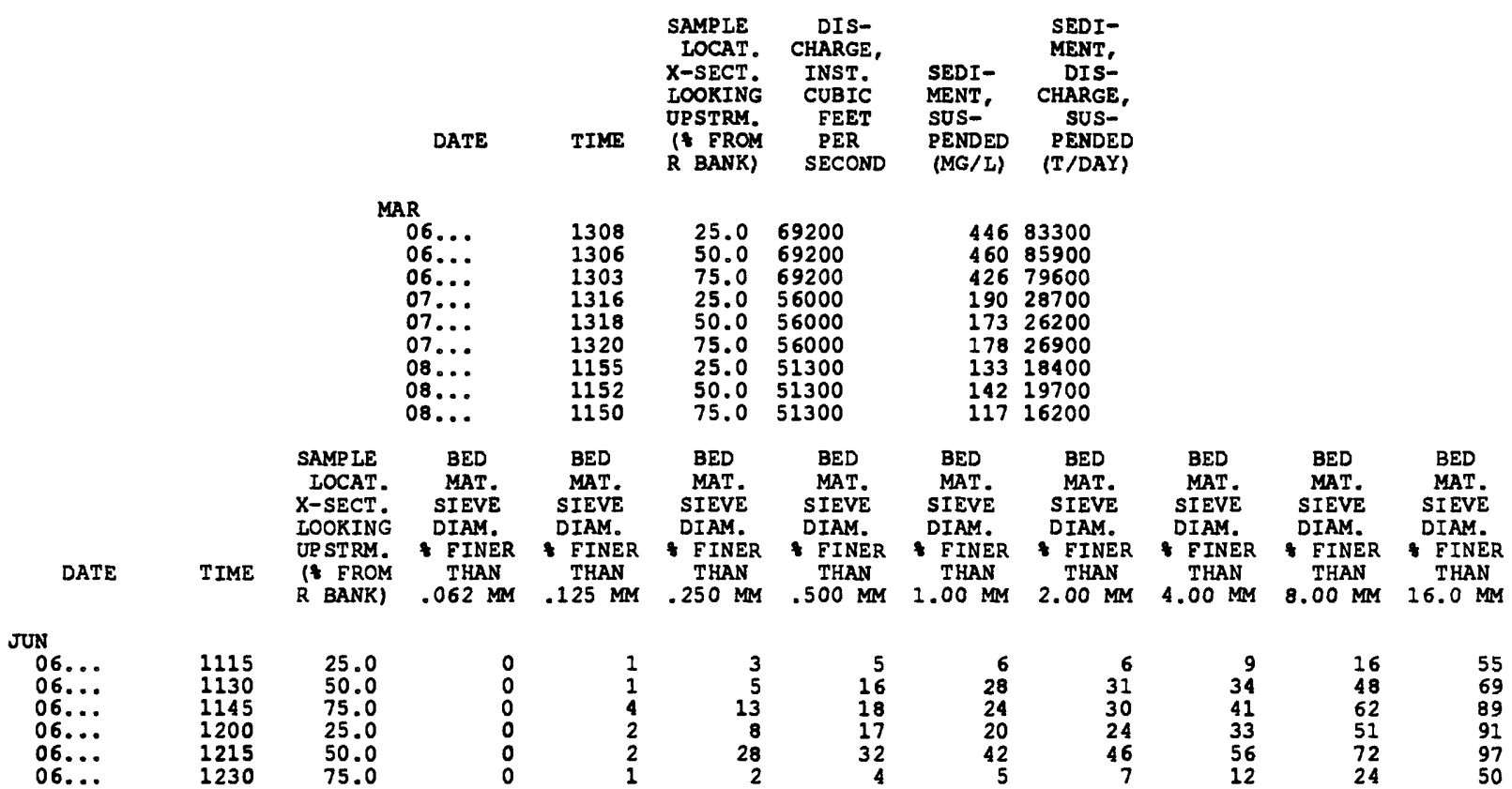


SURFACE-WATER SITES--Cont 1 nued

3321000882248 TTW ALICEVILLE LAKE HAIRSTON BEND SR $11 \mathrm{HB}$

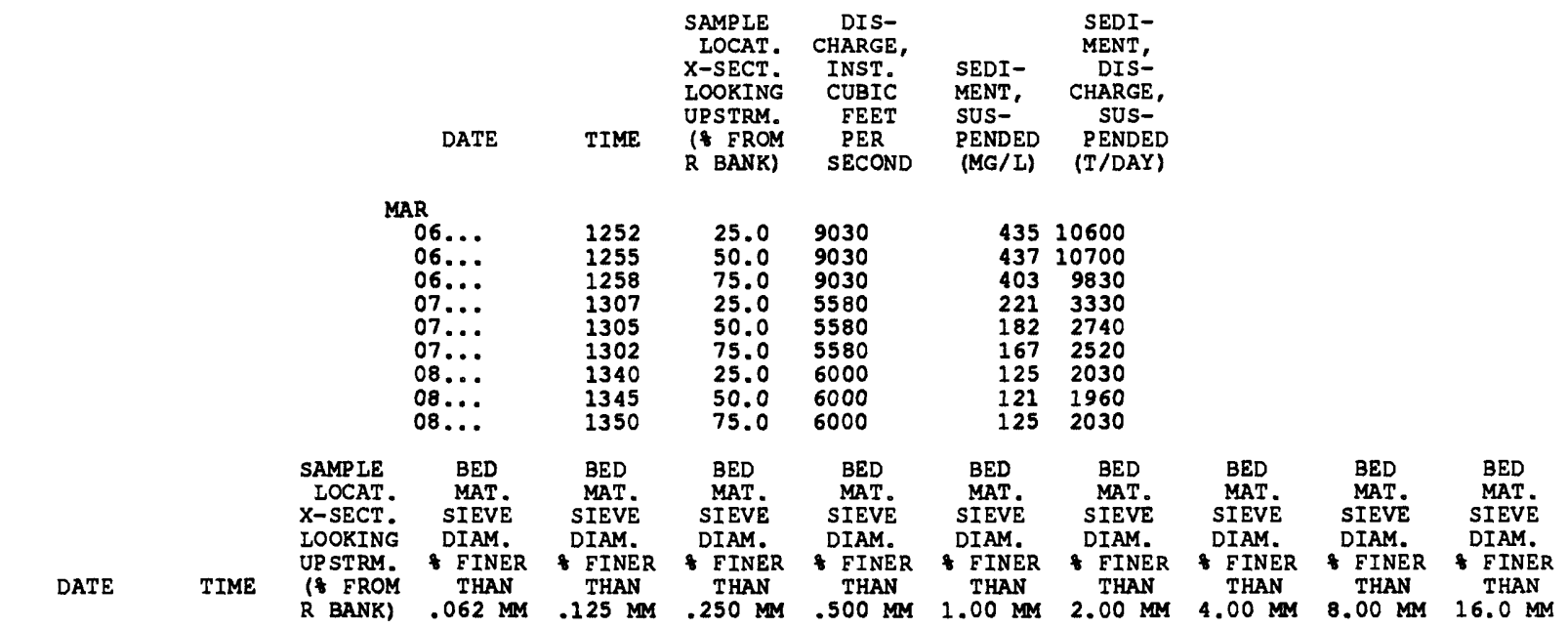

\begin{tabular}{rrrrrrrrrrr}
$06 . .$. & 1245 & 25.0 & 0 & 0 & 0 & 1 & 1 & 2 & 3 & 3 \\
$06 . .$. & 1300 & 50.0 & 1 & 3 & 8 & 23 & 30 & 35 & 40 & 48 \\
$06 . .$. & 1315 & 75.0 & 0 & 1 & 1 & 1 & 2 & 2 & 3 & 4 \\
\hline
\end{tabular}

02443610 TOMBIGBEE RIVER PRATT CAMP SR 5HB BELOW COLUMBUS, MS

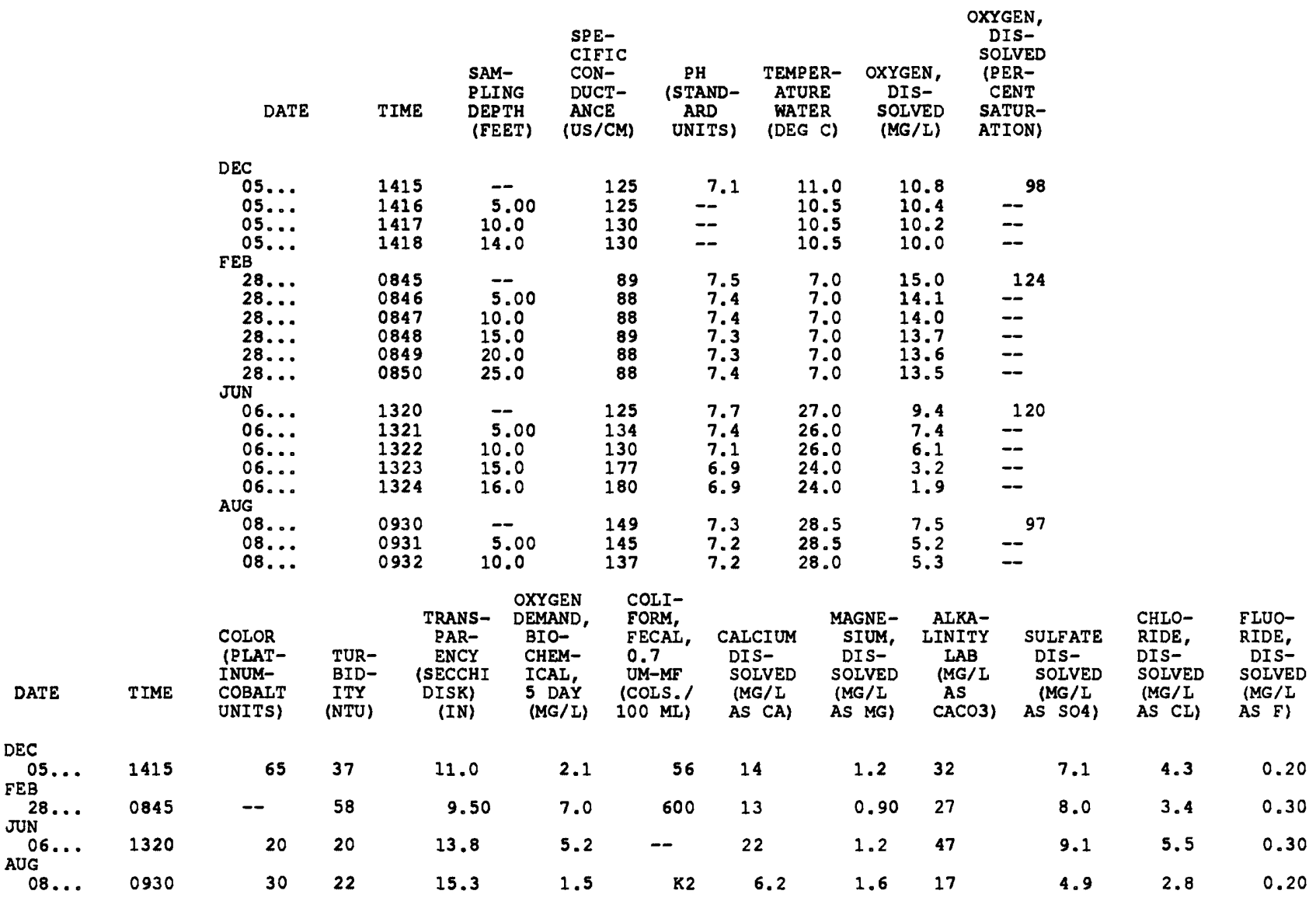


SURFACE-WATER SITES--Cont1 nued

02443610 TOMBIGBEE RIVER PRATT CAMP SR 5HB BELOW COLUMBUS, MS--Continued

\begin{tabular}{|c|c|c|c|c|c|c|c|c|c|c|c|c|}
\hline ATE & $\begin{array}{l}\text { SOLIDS, } \\
\text { RESIDUE } \\
\text { AT } 180 \\
\text { DEG. C } \\
\text { DIS- } \\
\text { SOIVED } \\
\text { (MG/L) }\end{array}$ & $\begin{array}{l}\text { RESIDUE } \\
\text { TOTAL } \\
\text { AT } 105 \\
\text { DEG. C, } \\
\text { SUS- } \\
\text { PENDED } \\
\text { (MG/L) }\end{array}$ & $\begin{array}{c}\text { NITRO- } \\
\text { GEN, } \\
\text { NO2+NO3 } \\
\text { DIS- } \\
\text { SOIVED } \\
\text { (MG/I } \\
\text { AS N) }\end{array}$ & $\begin{array}{c}\text { NITRO- } \\
\text { GEN, } \\
\text { AMMONIA } \\
\text { DIS- } \\
\text { SOLVED } \\
\text { (MG/L } \\
\text { AS N) }\end{array}$ & $\begin{array}{l}\text { NITRO- } \\
\text { GEN, AM- } \\
\text { MONIA + } \\
\text { ORGANIC } \\
\text { DIS } \\
\text { (MG/L } \\
\text { AS N) }\end{array}$ & $\begin{array}{l}\text { PHOS- } \\
\text { PHORUS } \\
\text { TOTAI } \\
\text { (MG/L } \\
\text { AS P) }\end{array}$ & $\begin{array}{c}\text { PHOS- } \\
\text { PHORUS } \\
\text { DIS- } \\
\text { SOLVED } \\
\text { (MG/L } \\
\text { AS P) }\end{array}$ & $\begin{array}{l}\text { IRON, } \\
\text { TOTAL } \\
\text { RECOV- } \\
\text { ERABLE } \\
\text { (UG/L } \\
\text { AS EE) }\end{array}$ & $\begin{array}{l}\text { MANGA- } \\
\text { NESE, } \\
\text { TOTAL } \\
\text { RECOV- } \\
\text { ERABLE } \\
\text { (UG/L } \\
\text { AS MN) }\end{array}$ & $\begin{array}{l}\text { PHENOLS } \\
\text { TOTAL } \\
\text { (UG/L) }\end{array}$ & $\begin{array}{c}\text { CHLOR-A } \\
\text { PHYTO- } \\
\text { PLANK- } \\
\text { TON } \\
\text { CHROMO } \\
\text { FLUOROM } \\
\text { (UG/L) }\end{array}$ & $\begin{array}{c}\text { CHLOR-B } \\
\text { P HYTO- } \\
\text { P LANK- } \\
\text { TON } \\
\text { CHROMO } \\
\text { FLUOROM } \\
\text { (UG/I) }\end{array}$ \\
\hline
\end{tabular}

\begin{tabular}{|c|c|c|c|c|c|c|c|c|c|c|c|c|}
\hline $\begin{array}{c}\mathrm{DES} \\
05 . . .\end{array}$ & 72 & 29 & 0.150 & 0.070 & 0.31 & 0.100 & 0.020 & - & - & 1 & -- & -- \\
\hline $28 \ldots$ & 63 & 86 & 0.180 & 0.040 & 0.33 & 0.150 & 0.020 & 2500 & 120 & $<1$ & 0.900 & 0.100 \\
\hline UG... & 102 & 34 & 0.210 & 0.030 & 0.34 & 0.100 & 0.030 & 1600 & 190 & $<1$ & 7.20 & 0.600 \\
\hline & 49 & 30 & 0.050 & 0.010 & 0.42 & 0.080 & 0.060 & 2700 & 470 & $<1$ & -- & -- \\
\hline
\end{tabular}

3320300882122 TTW ALICEVILLE LAKE HAIRSTON CUT

\begin{tabular}{|c|c|c|c|c|c|}
\hline DATE & TIME & $\begin{array}{l}\text { SAMPLE } \\
\text { LOCAT. } \\
\text { X-SECT. } \\
\text { LOOKING } \\
\text { UPSTRM. } \\
\text { (\& FROM } \\
\text { R BANK) }\end{array}$ & $\begin{array}{l}\text { DIS- } \\
\text { CHARGE, } \\
\text { INST. } \\
\text { CUBIC } \\
\text { FEET } \\
\text { PER } \\
\text { SECOND }\end{array}$ & $\begin{array}{l}\text { SEDI- } \\
\text { MENT, } \\
\text { SUS- } \\
\text { PENDED } \\
\text { (MG/L) }\end{array}$ & $\begin{array}{r}\text { SEDI } \\
\text { MENT } \\
\text { DIS } \\
\text { CHARG } \\
\text { SUS } \\
\text { PEND } \\
\text { (T/DA }\end{array}$ \\
\hline \multicolumn{6}{|l|}{ MAR } \\
\hline $\begin{array}{l}06 \ldots \\
06 \ldots \\
06 \ldots \\
07 \ldots \\
07 \ldots \\
07 \ldots \\
08 \ldots \\
08 \ldots \\
08 \ldots\end{array}$ & $\begin{array}{l}1319 \\
1317 \\
1315 \\
1238 \\
1237 \\
1233 \\
1315 \\
1320 \\
1325\end{array}$ & $\begin{array}{l}25.0 \\
50.0 \\
75.0 \\
25.0 \\
50.0 \\
75.0 \\
25.0 \\
50.0 \\
75.0\end{array}$ & $\begin{array}{l}60200 \\
60200 \\
60200 \\
50400 \\
50400 \\
50400 \\
45300 \\
45300 \\
45300\end{array}$ & $\begin{array}{l}422 \\
464 \\
460 \\
173 \\
176 \\
192 \\
118 \\
147 \\
126\end{array}$ & $\begin{array}{l}68600 \\
75400 \\
74800 \\
23500 \\
24000 \\
26100 \\
14400 \\
18000 \\
15400\end{array}$ \\
\hline
\end{tabular}

02444157 TOMBIGBEE RIVER AT ST HKY 86 NEAR RICKENSVILLE, AL

\begin{tabular}{|c|c|c|c|c|c|}
\hline DATE & TIME & $\begin{array}{l}\text { SAMPLE } \\
\text { LOCAT. } \\
\text { X-SECT. } \\
\text { LOOKING } \\
\text { UPSTRM. } \\
\text { (\& FROM } \\
\text { R BANK) }\end{array}$ & $\begin{array}{l}\text { DIS- } \\
\text { CHARGE, } \\
\text { INST. } \\
\text { CUBIC } \\
\text { FEET } \\
\text { PER } \\
\text { SECOND }\end{array}$ & $\begin{array}{l}\text { SEDI- } \\
\text { MENT, } \\
\text { SUS- } \\
\text { PENDED } \\
\text { (MG/L) }\end{array}$ & $\begin{array}{r}\text { SEDI- } \\
\text { MENT, } \\
\text { DIS- } \\
\text { CHARGE } \\
\text { SUS- } \\
\text { PENDE } \\
\text { (T/DAY) }\end{array}$ \\
\hline \multicolumn{6}{|l|}{$\infty r$} \\
\hline $\begin{array}{r}21 \ldots \\
21 \ldots \\
21 \ldots \\
\text { DEC }\end{array}$ & $\begin{array}{l}1115 \\
1125 \\
1130\end{array}$ & $\begin{array}{l}25.0 \\
50.0 \\
75.0\end{array}$ & $\begin{array}{l}4600 \\
4600 \\
4600\end{array}$ & $\begin{array}{l}32 \\
19 \\
22\end{array}$ & $\begin{array}{l}397 \\
236 \\
273\end{array}$ \\
\hline $\begin{array}{r}07 \ldots \\
07 \ldots \\
07 \ldots \\
\text { JAN }\end{array}$ & $\begin{array}{l}1430 \\
1435 \\
1440\end{array}$ & $\begin{array}{l}25.0 \\
50.0 \\
75.0\end{array}$ & $\begin{array}{l}3500 \\
3500 \\
3500\end{array}$ & $\begin{array}{l}28 \\
45 \\
40\end{array}$ & $\begin{array}{l}265 \\
425 \\
378\end{array}$ \\
\hline $\begin{array}{r}12 \ldots \\
12 \ldots \\
12 \ldots \\
M A R\end{array}$ & $\begin{array}{l}1445 \\
1500 \\
1510\end{array}$ & $\begin{array}{l}25.0 \\
50.0 \\
75.0\end{array}$ & $\begin{array}{l}54500 \\
54500 \\
54500\end{array}$ & $\begin{array}{l}361 \\
275 \\
416\end{array}$ & $\begin{array}{l}53100 \\
40500 \\
61200\end{array}$ \\
\hline $\begin{array}{r}01 \ldots \\
01 \ldots \\
01 \ldots \\
\text { APR }\end{array}$ & $\begin{array}{l}1130 \\
1140 \\
1145\end{array}$ & $\begin{array}{l}25.0 \\
50.0 \\
75.0\end{array}$ & $\begin{array}{l}64500 \\
64500 \\
64500\end{array}$ & $\begin{array}{l}329 \\
292 \\
310\end{array}$ & $\begin{array}{l}57300 \\
50900 \\
54000\end{array}$ \\
\hline $\begin{array}{r}12 \ldots \\
12 \ldots \\
12 \ldots \\
M A Y\end{array}$ & $\begin{array}{l}0950 \\
0955 \\
1000\end{array}$ & $\begin{array}{l}25.0 \\
50.0 \\
75.0\end{array}$ & $\begin{array}{l}7870 \\
7870 \\
7870\end{array}$ & $\begin{array}{l}13 \\
16 \\
11\end{array}$ & $\begin{array}{l}276 \\
340 \\
234\end{array}$ \\
\hline $\begin{array}{l}23 \ldots \\
23 \ldots \\
23 \ldots \\
\text { Jut }\end{array}$ & $\begin{array}{l}1000 \\
1010 \\
1015\end{array}$ & $\begin{array}{l}25.0 \\
50.0 \\
75.0\end{array}$ & $\begin{array}{l}9570 \\
9570 \\
9570\end{array}$ & $\begin{array}{l}17 \\
27 \\
29\end{array}$ & $\begin{array}{l}439 \\
698 \\
749\end{array}$ \\
\hline $\begin{array}{c}05 \ldots \\
05 \ldots \\
05 \ldots \\
\text { AUG }\end{array}$ & $\begin{array}{l}1030 \\
1035 \\
1045\end{array}$ & $\begin{array}{l}25.0 \\
50.0 \\
75.0\end{array}$ & $\begin{array}{l}26600 \\
26600 \\
26600\end{array}$ & $\begin{array}{l}71 \\
50 \\
96\end{array}$ & $\begin{array}{l}5100 \\
3590 \\
6890\end{array}$ \\
\hline $\begin{array}{l}15 \ldots \\
15 \ldots \\
15 \ldots\end{array}$ & $\begin{array}{l}1020 \\
1025 \\
1030\end{array}$ & $\begin{array}{l}25.0 \\
50.0 \\
75.0\end{array}$ & $\begin{array}{l}3700 \\
3700 \\
3700\end{array}$ & $\begin{array}{r}8 \\
10 \\
6\end{array}$ & $\begin{array}{r}80 \\
100 \\
60\end{array}$ \\
\hline
\end{tabular}


02444210 TOMBIGBEE RIVER BIG CREEK BEND NEAR PICKENSVILIE, AL

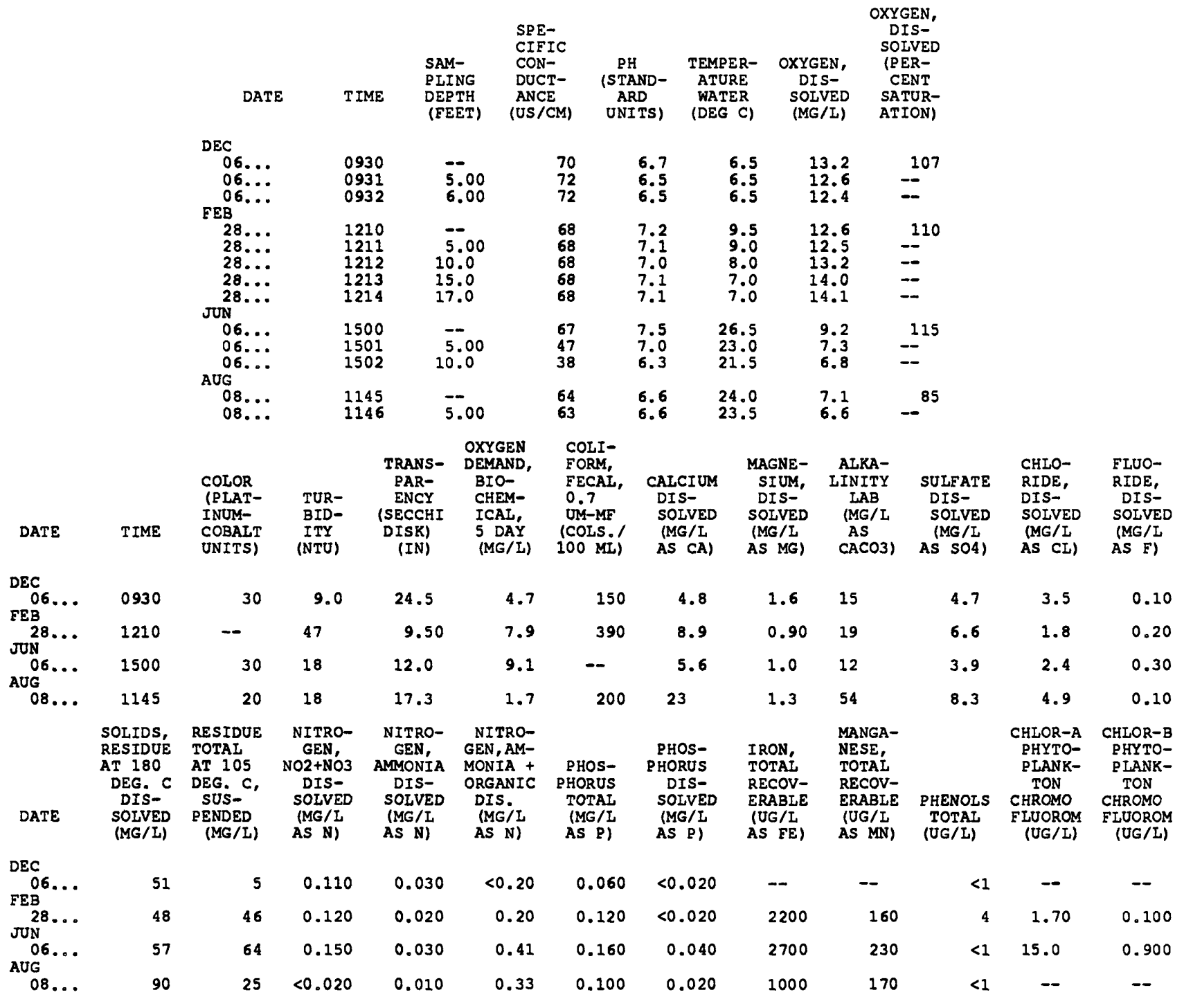


SURFACE-WATER SITES--Cont 1 nued

02444500 TOMBIGBEE RIVER NEAR COCHRANE, AL

\begin{tabular}{|c|c|c|c|c|c|}
\hline DATE & TIME & $\begin{array}{l}\text { SAMPLE } \\
\text { LOCAT. } \\
\text { X-SECT. } \\
\text { IOOKING } \\
\text { UPSTRM. } \\
\text { (\& FROM } \\
\text { R BANK) }\end{array}$ & $\begin{array}{c}\text { DIS- } \\
\text { CHARGE, } \\
\text { INST. } \\
\text { CUBIC } \\
\text { FEET } \\
\text { PER } \\
\text { SECOND }\end{array}$ & $\begin{array}{l}\text { SEDI- } \\
\text { MENT, } \\
\text { SUS- } \\
\text { PENDED } \\
\text { (MG/L) }\end{array}$ & $\begin{array}{c}\text { SEDI- } \\
\text { MENT, } \\
\text { DIS- } \\
\text { CHARGE } \\
\text { SUS- } \\
\text { PENDEI } \\
\text { (T/DAY) }\end{array}$ \\
\hline \multicolumn{6}{|l|}{ OCT } \\
\hline $\begin{array}{r}21 \ldots \\
21 \ldots \\
21 \ldots \\
D E C\end{array}$ & $\begin{array}{l}1250 \\
1300 \\
1305\end{array}$ & $\begin{array}{l}25.0 \\
50.0 \\
75.0\end{array}$ & $\begin{array}{l}2170 \\
2170 \\
2170\end{array}$ & $\begin{array}{l}86 \\
20 \\
20\end{array}$ & $\begin{array}{l}504 \\
117 \\
117\end{array}$ \\
\hline $\begin{array}{r}08 \ldots \\
08 \ldots \\
08 \ldots \\
\text { JAN }\end{array}$ & $\begin{array}{l}1425 \\
1430 \\
1440\end{array}$ & $\begin{array}{l}25.0 \\
50.0 \\
75.0\end{array}$ & $\begin{array}{l}4380 \\
4380 \\
4380\end{array}$ & $\begin{array}{l}15 \\
15 \\
16\end{array}$ & $\begin{array}{l}177 \\
177 \\
189\end{array}$ \\
\hline $\begin{array}{r}12 \ldots \\
12 \ldots \\
12 \ldots \\
M A R\end{array}$ & $\begin{array}{l}1615 \\
1620 \\
1630\end{array}$ & $\begin{array}{l}25.0 \\
50.0 \\
75.0\end{array}$ & $\begin{array}{l}\mathbf{5 5 3 0 0} \\
\mathbf{5 5 3 0 0} \\
\mathbf{5 5 3 0 0}\end{array}$ & $\begin{array}{l}355 \\
358 \\
409\end{array}$ & $\begin{array}{l}53000 \\
53500 \\
61100\end{array}$ \\
\hline $\begin{array}{r}01 \ldots \\
01 \ldots \\
01 \ldots \\
\text { APR }\end{array}$ & $\begin{array}{l}1310 \\
1315 \\
1320\end{array}$ & $\begin{array}{l}25.0 \\
50.0 \\
75.0\end{array}$ & $\begin{array}{l}64500 \\
64500 \\
64500\end{array}$ & $\begin{array}{l}325 \\
359 \\
437\end{array}$ & $\begin{array}{l}56600 \\
62500 \\
76100\end{array}$ \\
\hline $\begin{array}{r}12 \ldots \\
12 \ldots \\
12 \ldots \\
M A Y\end{array}$ & $\begin{array}{l}1121 \\
1130 \\
1135\end{array}$ & $\begin{array}{l}25.0 \\
50.0 \\
75.0\end{array}$ & $\begin{array}{l}8930 \\
8930 \\
8930\end{array}$ & $\begin{array}{r}11 \\
6 \\
11\end{array}$ & $\begin{array}{l}265 \\
145 \\
265\end{array}$ \\
\hline $\begin{array}{r}23 \ldots \\
23 \ldots \\
23 \ldots \\
\text { JUL }\end{array}$ & $\begin{array}{l}1105 \\
1110 \\
1115\end{array}$ & $\begin{array}{l}25.0 \\
50.0 \\
75.0\end{array}$ & $\begin{array}{l}9990 \\
9990 \\
9990\end{array}$ & $\begin{array}{l}19 \\
26 \\
32\end{array}$ & $\begin{array}{l}512 \\
701 \\
863\end{array}$ \\
\hline $\begin{array}{l}05 \ldots \\
05 \ldots \\
05 \ldots \\
\text { AUG }\end{array}$ & $\begin{array}{l}1140 \\
1150 \\
1155\end{array}$ & $\begin{array}{l}25.0 \\
50.0 \\
75.0\end{array}$ & $\begin{array}{l}27200 \\
27200 \\
27200\end{array}$ & $\begin{array}{l}78 \\
72 \\
64\end{array}$ & $\begin{array}{l}5730 \\
5290 \\
4700\end{array}$ \\
\hline $\begin{array}{l}15 \ldots \\
15 \ldots \\
15 \ldots\end{array}$ & $\begin{array}{l}1130 \\
1135 \\
1140\end{array}$ & $\begin{array}{l}25.0 \\
50.0 \\
75.0\end{array}$ & $\begin{array}{l}3420 \\
3420 \\
3420\end{array}$ & $\begin{array}{l}12 \\
14 \\
14\end{array}$ & $\begin{array}{l}111 \\
129 \\
129\end{array}$ \\
\hline
\end{tabular}

02446500 SIPSEY RIVER NEAR ELROD, AL

\begin{tabular}{|c|c|c|c|c|c|c|c|c|c|}
\hline & DATE & TIME & $\begin{array}{l}\text { SAM- } \\
\text { PIING } \\
\text { DEPTH } \\
\text { (FEET) }\end{array}$ & $\begin{array}{l}\text { SPE- } \\
\text { CIFIC } \\
\text { CON- } \\
\text { DUCT- } \\
\text { ANCE } \\
\text { (US/CM) }\end{array}$ & $\begin{array}{c}\text { PH } \\
\text { (STAND- } \\
\text { ARD } \\
\text { UNITS) }\end{array}$ & $\begin{array}{c}\text { TEMPER- } \\
\text { ATURE } \\
\text { WATER } \\
\text { (DEG C) }\end{array}$ & $\begin{array}{c}\text { OXYGEN, } \\
\text { DIS- } \\
\text { SOLVED } \\
\text { (MG/I) }\end{array}$ & $\begin{array}{c}\text { OXYGEN, } \\
\text { DIS- } \\
\text { SOLVED } \\
\text { (PER- } \\
\text { CENT } \\
\text { SATUR- } \\
\text { ATION) }\end{array}$ & \\
\hline & $\begin{array}{l}\text { DEC } \\
05 \ldots \\
05 \ldots \\
05 \ldots \\
\text { FEB }\end{array}$ & $\begin{array}{l}0915 \\
0916 \\
0917\end{array}$ & $\begin{array}{l}5.00 \\
8.00\end{array}$ & $\begin{array}{l}70 \\
70 \\
70\end{array}$ & $\begin{array}{l}6.2 \\
6.2 \\
6.2\end{array}$ & $\begin{array}{l}7.5 \\
7.5 \\
7.5\end{array}$ & $\begin{array}{l}9.9 \\
9.6 \\
9.6\end{array}$ & $\begin{array}{l}82 \\
--\end{array}$ & \\
\hline & $\begin{array}{r}27 \ldots \\
27 \ldots \\
27 \ldots \\
27 \ldots \\
\text { JUN }\end{array}$ & $\begin{array}{l}0940 \\
0941 \\
0942 \\
0943\end{array}$ & $\begin{array}{c}\overline{5.00} \\
10.0 \\
12.0\end{array}$ & $\begin{array}{l}52 \\
52 \\
52 \\
52\end{array}$ & $\begin{array}{l}7.3 \\
6.7 \\
6.4 \\
6.4\end{array}$ & $\begin{array}{l}8.5 \\
8.0 \\
8.0 \\
8.0\end{array}$ & $\begin{array}{l}11.9 \\
12.0 \\
11.3 \\
10.9\end{array}$ & $\begin{array}{l}103 \\
= \\
=\end{array}$ & \\
\hline & $\begin{array}{l}05 \ldots \\
05 \ldots \\
\text { AUG }\end{array}$ & $\begin{array}{l}0915 \\
0916\end{array}$ & $\overline{4.00}$ & $\begin{array}{l}85 \\
84\end{array}$ & $\begin{array}{l}6.4 \\
6.4\end{array}$ & $\begin{array}{l}23.5 \\
23.5\end{array}$ & $\begin{array}{l}6.5 \\
6.4\end{array}$ & -77 & \\
\hline & $\begin{array}{l}07 \ldots \\
07 \ldots\end{array}$ & $\begin{array}{l}0945 \\
0946\end{array}$ & -- & $\begin{array}{l}90 \\
91\end{array}$ & $\begin{array}{l}8.4 \\
7.8\end{array}$ & $\begin{array}{l}26.5 \\
26.5\end{array}$ & $\begin{array}{l}6.0 \\
5.5\end{array}$ & $--^{76}$ & \\
\hline DATE & TIME & $\begin{array}{c}\text { DIS- } \\
\text { CHARGE, } \\
\text { INST. } \\
\text { CUBIC } \\
\text { FEET } \\
\text { PER } \\
\text { SECOND }\end{array}$ & $\begin{array}{l}\text { COLOR } \\
\text { (PLAT- } \\
\text { INUM- } \\
\text { COBALT } \\
\text { UNITS) }\end{array}$ & $\begin{array}{c}\text { TUR- } \\
\text { BID- } \\
\text { ITY } \\
\text { (NTU) }\end{array}$ & $\begin{array}{c}\text { TRANS- } \\
\text { PAR- } \\
\text { ENCY } \\
\text { (SECCHI } \\
\text { DISK) } \\
\text { (IN) }\end{array}$ & $\begin{array}{l}\text { OXYGEN } \\
\text { DEMAND, } \\
\text { BIO- } \\
\text { CHEM- } \\
\text { ICAL, } \\
5 \text { DAY } \\
\text { (MG/L) }\end{array}$ & $\begin{array}{l}\text { COLI- } \\
\text { FORM, } \\
\text { FECAL, } \\
0.7 \\
\text { UM-MF } \\
\text { (COLS.' } \\
\text { IOO ML) }\end{array}$ & $\begin{array}{l}\text { CALCIUM } \\
\text { DIS- } \\
\text { SOLVED } \\
\text { (MG/I } \\
\text { AS CA) }\end{array}$ & $\begin{array}{l}\text { MAGNE- } \\
\text { SIUM, } \\
\text { DIS- } \\
\text { SOLVED } \\
\text { (MG/L } \\
\text { AS MG) }\end{array}$ \\
\hline \multicolumn{10}{|l|}{ DEC } \\
\hline FEB & 0915 & 1270 & 30 & 4.1 & 39.8 & 3.8 & 130 & 4.2 & 2.9 \\
\hline$\sigma_{N}^{27} \ldots$ & 0940 & 2890 & 40 & 11 & 30.0 & 1.8 & 230 & 3.1 & 2.2 \\
\hline$\underset{A U G}{05 \ldots}$ & 0915 & 215 & 40 & 10 & 30.3 & 0.4 & 470 & 6.0 & 4.0 \\
\hline $07 \ldots$ & 0945 & 255 & 40 & 11 & 31.5 & 5.0 & 47 & 7.3 & 4.5 \\
\hline
\end{tabular}


SURFACE-WATER SITES--Continued

02446500 SIPSEY RIVER NEAR ELROD, AL--Cont1 nued

\begin{tabular}{|c|c|c|c|c|c|c|c|c|}
\hline DATE & $\begin{array}{c}\text { ALKA- } \\
\text { LINITY } \\
\text { LAB } \\
\text { (MG/L } \\
\text { AS } \\
\text { CACO3) }\end{array}$ & $\begin{array}{l}\text { SULFATE } \\
\text { DIS- } \\
\text { SOLVED } \\
\text { (MG/L } \\
\text { AS SO4) }\end{array}$ & $\begin{array}{l}\text { CHLO- } \\
\text { RIDE, } \\
\text { DIS- } \\
\text { SOLVED } \\
\text { (MG/L } \\
\text { AS CL) }\end{array}$ & $\begin{array}{l}\text { FLUO- } \\
\text { RIDE, } \\
\text { DIS- } \\
\text { SOLVED } \\
\text { (MG/L } \\
\text { AS F) }\end{array}$ & $\begin{array}{l}\text { SOLIDS, } \\
\text { RESIDUE } \\
\text { AT } 180 \\
\text { DEG. C } \\
\text { DIS- } \\
\text { SOLVED } \\
\text { (MG/L) }\end{array}$ & $\begin{array}{l}\text { RESIDUE } \\
\text { TOTAL } \\
\text { AT } 105 \\
\text { DEG. C, } \\
\text { SUS- } \\
\text { PENDED } \\
\text { (MG/L) }\end{array}$ & $\begin{array}{c}\text { NITRO- } \\
\text { GEN, } \\
\text { NO2+NO3 } \\
\text { DIS- } \\
\text { SOLVED } \\
\text { (MG/I } \\
\text { AS N) }\end{array}$ & $\begin{array}{l}\text { NITRO- } \\
\text { GEN, } \\
\text { AMMONIA } \\
\text { DIS- } \\
\text { SOLVED } \\
\text { (MG/I } \\
\text { AS N) }\end{array}$ \\
\hline $\begin{array}{l}\mathrm{DEC} \\
05 \ldots \\
\text { FEB }\end{array}$ & 6.2 & 18 & 2.3 & 0.20 & 60 & $<1$ & 0.050 & 0.010 \\
\hline JUN $27 \ldots$ & 4.4 & 13 & 1.9 & 0.10 & 42 & 6 & 0.020 & $<0.010$ \\
\hline UG $05 .$. & 17 & 14 & 2.1 & 0.20 & 65 & 18 & 0.190 & 0.050 \\
\hline $07 \ldots$ & 20 & 16 & 2.2 & 0.10 & 62 & 10 & 0.230 & 0.050 \\
\hline DATE & $\begin{array}{l}\text { NITRO- } \\
\text { GEN, AM- } \\
\text { MONIA + } \\
\text { ORGANIC } \\
\text { DIS. } \\
\text { (MG/I } \\
\text { AS N) }\end{array}$ & $\begin{array}{l}\text { PHOS- } \\
\text { PHORUS } \\
\text { TOTAL } \\
\text { (MG/L } \\
\text { AS P) }\end{array}$ & $\begin{array}{l}\text { PHOS- } \\
\text { PHORUS } \\
\text { DIS- } \\
\text { SOLVED } \\
\text { (MG/I } \\
\text { AS P) }\end{array}$ & $\begin{array}{l}\text { IRON, } \\
\text { TOTAL } \\
\text { RECOV- } \\
\text { ERABLE } \\
\text { (UG/L } \\
\text { AS FE) }\end{array}$ & $\begin{array}{l}\text { MANGA- } \\
\text { NESE, } \\
\text { TOTAL } \\
\text { RECOV- } \\
\text { ERABLE } \\
\text { (UG/L } \\
\text { AS MN) }\end{array}$ & $\begin{array}{l}\text { PHENOLS } \\
\text { TOTAL } \\
\text { (UG/L) }\end{array}$ & $\begin{array}{l}\text { CHLOR-A } \\
\text { PHYTO- } \\
\text { PLANK- } \\
\text { TON } \\
\text { CHROMO } \\
\text { FLUOROM } \\
\text { (UG/I) }\end{array}$ & $\begin{array}{c}\text { CHLOR-B } \\
\text { PHYTO- } \\
\text { PIANK- } \\
\text { TON } \\
\text { CHROMO } \\
\text { FLUOROM } \\
\text { (UG/I) }\end{array}$ \\
\hline
\end{tabular}

\begin{tabular}{|c|c|c|c|c|c|c|c|c|}
\hline $\begin{array}{l}\text { DEC } \\
05 \ldots \\
\text { FEB }\end{array}$ & 0.23 & 0.040 & 0.040 & - & - & $<1$ & - & -- \\
\hline JUN $27 \ldots$ & 0.27 & 0.030 & $<0.020$ & 1000 & 37 & 5 & 0.700 & 0.100 \\
\hline$\underset{A U G}{05}$ & 0.21 & 0.050 & 0.030 & 2000 & 500 & $<1$ & 1.10 & 0.200 \\
\hline $07 \ldots$ & 0.23 & 0.040 & $<0.020$ & 1700 & 600 & $<1$ & -- & -- \\
\hline
\end{tabular}

02447008 TOMBIGBEE RIVER ABOVE COOKS BEND SR 5A NEAR WARSAW, AL

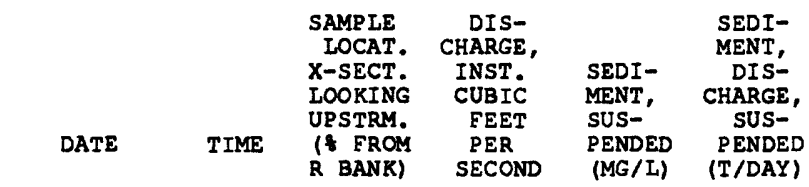

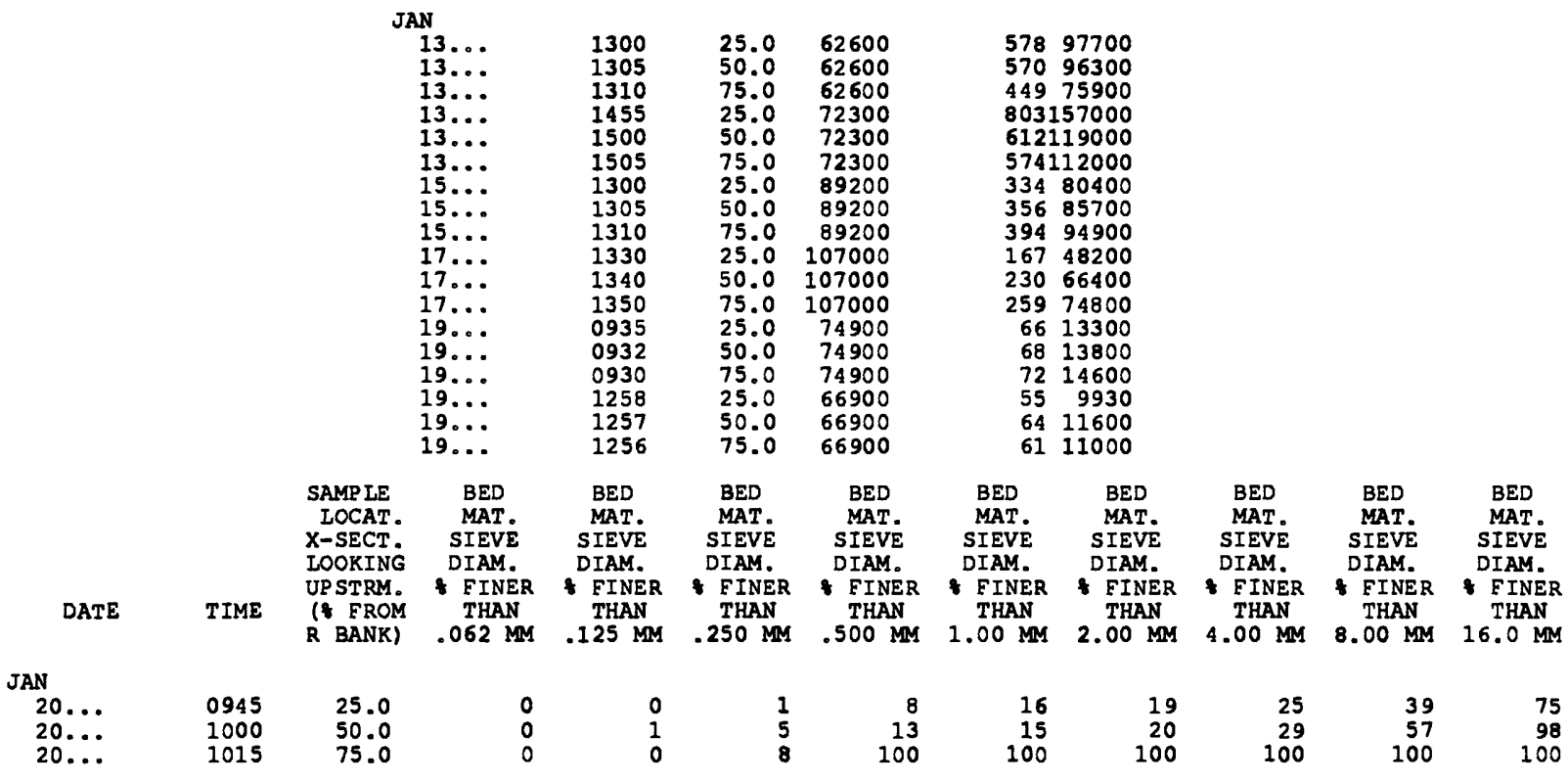


SURFACE-WATER SITES--Cont I nued

02447010 TOMBIGBEE RIVER COOKS BEND NEAR WARSAW, AL

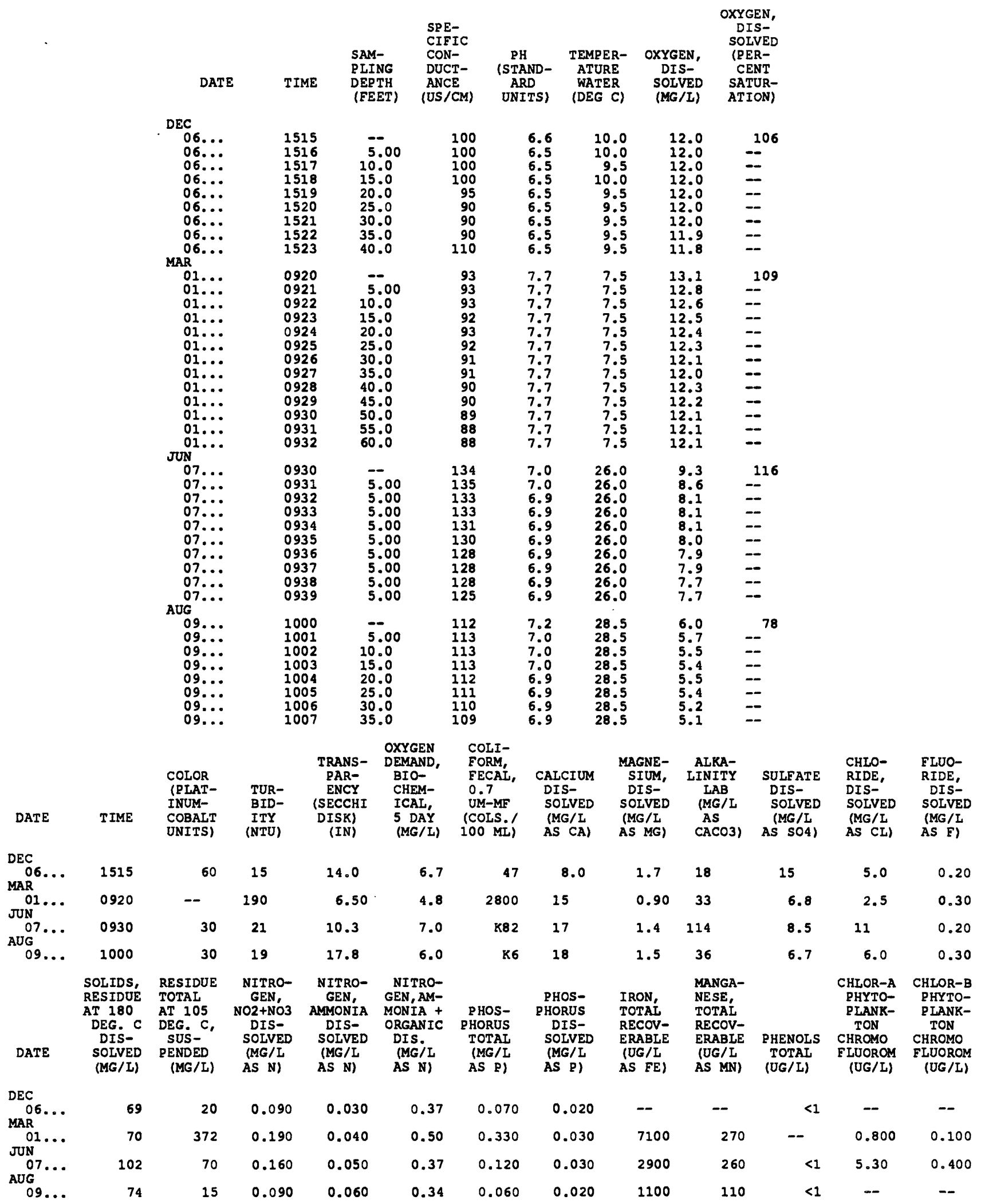


SURFACE-WATER SITES--Cont Inued

02447010 TOMBIGBEE RIVER COOKS BEND NEAR WARSAW, AL--ContI nued

\begin{tabular}{|c|c|c|c|c|c|c|c|c|c|c|c|}
\hline & & & DATE & TIME & $\begin{array}{l}\text { SAMPLE } \\
\text { LOCAT. } \\
\text { X-SECT. } \\
\text { LOOKING } \\
\text { UP STRM. } \\
\text { (\$ FROM } \\
\text { R BANK) }\end{array}$ & $\begin{array}{c}\text { DIS- } \\
\text { CHARGE, } \\
\text { INST. } \\
\text { CUBIC } \\
\text { FEET } \\
\text { PER } \\
\text { SECOND }\end{array}$ & $\begin{array}{l}\text { SEDI- } \\
\text { MENT, } \\
\text { SUS- } \\
\text { PENDED } \\
\text { (MG/L) }\end{array}$ & $\begin{array}{c}\text { SEDI- } \\
\text { MENT, } \\
\text { DIS- } \\
\text { CHARGE, } \\
\text { SUS- } \\
\text { PENDED } \\
\text { (T/DAY) }\end{array}$ & & & \\
\hline & & & $\begin{array}{l}\mathbb{1 N} \\
13 \ldots \\
13 \ldots \\
13 \ldots \\
13 \ldots \\
13 \ldots \\
13 \ldots \\
15 \ldots \\
15 \ldots \\
15 \ldots \\
17 \ldots \\
17 \ldots \\
17 \ldots \\
19 \ldots \\
19 \ldots \\
19 \ldots \\
19 \ldots\end{array}$ & $\begin{array}{l}1325 \\
1330 \\
1335 \\
1525 \\
1530 \\
1535 \\
1250 \\
1255 \\
1300 \\
1320 \\
1325 \\
1330 \\
0927 \\
0926 \\
0922 \\
1254 \\
1253 \\
1251\end{array}$ & $\begin{array}{l}25.0 \\
50.0 \\
75.0 \\
25.0 \\
50.0 \\
75.0 \\
25.0 \\
50.0 \\
75.0 \\
25.0 \\
50.0 \\
75.0 \\
25.0 \\
50.0 \\
75.0 \\
25.0 \\
50.0 \\
75.0\end{array}$ & $\begin{array}{l}36500 \\
36500 \\
36500 \\
32900 \\
32900 \\
32900 \\
36400 \\
36400 \\
36400 \\
49500 \\
49500 \\
49500 \\
34900 \\
34900 \\
34900 \\
29100 \\
29100 \\
29100\end{array}$ & $\begin{array}{r}625 \\
670 \\
846 \\
547 \\
700 \\
802 \\
299 \\
436 \\
523 \\
222 \\
231 \\
253 \\
68 \\
91 \\
118 \\
57 \\
156 \\
77\end{array}$ & $\begin{array}{r}61600 \\
66000 \\
83400 \\
48600 \\
62200 \\
71200 \\
29400 \\
42900 \\
51400 \\
29700 \\
30900 \\
33800 \\
6410 \\
8570 \\
11100 \\
4480 \\
12300 \\
6050\end{array}$ & & & \\
\hline DATE & TIME & $\begin{array}{l}\text { SAMPLE } \\
\text { LOCAT. } \\
\text { X-SECT. } \\
\text { LOOKING } \\
\text { UP STRM. } \\
\text { (\& FROM } \\
\text { R BANK) }\end{array}$ & $\begin{array}{c}\text { BED } \\
\text { MAT. } \\
\text { SIEVE } \\
\text { DIAM. } \\
+ \text { FINER } \\
\text { THAN } \\
.062 \mathrm{MM}\end{array}$ & $\begin{array}{c}\text { BED } \\
\text { MAT. } \\
\text { SIEVE } \\
\text { DIAM. } \\
\text { FINER } \\
\text { THAN } \\
.125 \mathrm{MM}\end{array}$ & $\begin{array}{c}\text { BED } \\
\text { MAT. } \\
\text { SIEVE } \\
\text { DIAM. } \\
\text { FINER } \\
\text { THAN } \\
.250 \text { MM }\end{array}$ & $\begin{array}{c}\text { BED } \\
\text { MAT. } \\
\text { SIEVE } \\
\text { DIAM. } \\
\text { \& FINER } \\
\text { THAN } \\
.500 \mathrm{MM}\end{array}$ & $\begin{array}{c}\text { BED } \\
\text { MAT. } \\
\text { SIEVE } \\
\text { DIAM. } \\
+ \text { FINER } \\
\text { THAN } \\
1.00 \mathrm{MM}\end{array}$ & $\begin{array}{c}\text { BED } \\
\text { MAT. } \\
\text { SIEVE } \\
\text { DIAM. } \\
\text { FINER } \\
\text { THAN } \\
2.00 \mathrm{MM}\end{array}$ & $\begin{array}{c}\text { BED } \\
\text { MAT. } \\
\text { SIEVE } \\
\text { DIAM. } \\
\text { FINER } \\
\text { THAN } \\
4.00 \mathrm{MM}\end{array}$ & $\begin{array}{c}\text { BED } \\
\text { MAT. } \\
\text { SIEVE } \\
\text { DIAM. } \\
\text { FINER } \\
\text { THAN } \\
8.00 \mathrm{MM}\end{array}$ & $\begin{array}{c}\text { BED } \\
\text { MAT. } \\
\text { SIEVE } \\
\text { DIAM. } \\
\text { F EINER } \\
\text { THAN } \\
16.0 \mathrm{MM}\end{array}$ \\
\hline $\begin{array}{l}0 . . . \\
0 . . . \\
0 . .\end{array}$ & $\begin{array}{l}0900 \\
0910 \\
0920\end{array}$ & $\begin{array}{l}25.0 \\
50.0 \\
75.0\end{array}$ & $\begin{array}{l}0 \\
0 \\
4\end{array}$ & $\begin{array}{l}0 \\
2 \\
8\end{array}$ & $\begin{array}{l}34 \\
50 \\
23\end{array}$ & $\begin{array}{r}100 \\
95 \\
12\end{array}$ & $\begin{array}{r}100 \\
95 \\
14\end{array}$ & $\begin{array}{r}100 \\
96 \\
16\end{array}$ & $\begin{array}{r}100 \\
96 \\
20\end{array}$ & $\begin{array}{r}100 \\
97 \\
23\end{array}$ & $\begin{array}{r}100 \\
100 \\
30\end{array}$ \\
\hline
\end{tabular}

325645088100700 TTW GAINESVILIE LAKE COOKS BEND CUT SR 4AD

\begin{tabular}{|c|c|c|c|c|c|c|c|c|c|c|c|}
\hline & & & DATE & TIME & $\begin{array}{l}\text { SAMPLE } \\
\text { IOCAT. } \\
\text { X-SECT. } \\
\text { LOOKING } \\
\text { UPSTRM. } \\
\text { (\& FROM } \\
\text { R BANK) }\end{array}$ & $\begin{array}{c}\text { DIS- } \\
\text { CHARGE, } \\
\text { INST. } \\
\text { CUBIC } \\
\text { FEET } \\
\text { PER } \\
\text { SECOND }\end{array}$ & $\begin{array}{l}\text { SEDI- } \\
\text { MENT, } \\
\text { SUS- } \\
\text { PENDED } \\
\text { (MG/L) }\end{array}$ & $\begin{array}{c}\text { SEDI- } \\
\text { MENT, } \\
\text { DIS- } \\
\text { CHARGE, } \\
\text { SUS- } \\
\text { PENDED } \\
\text { (T/DAY) }\end{array}$ & & & \\
\hline 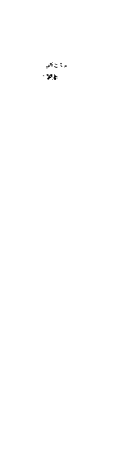 & & & $\begin{array}{l}1 \mathrm{~N} \\
13 \ldots \\
13 \ldots \\
13 \ldots \\
13 \ldots \\
13 \ldots \\
13 \ldots \\
15 \ldots \\
15 \ldots \\
15 \ldots \\
17 \ldots \\
17 \ldots \\
17 \ldots \\
19 \ldots \\
19 \ldots \\
19 \ldots \\
19 \ldots \\
19 \ldots\end{array}$ & $\begin{array}{l}1315 \\
1320 \\
1325 \\
1505 \\
1510 \\
1515 \\
1245 \\
1250 \\
1255 \\
1305 \\
1310 \\
1315 \\
0936 \\
0939 \\
0942 \\
1259 \\
1304 \\
1306\end{array}$ & $\begin{array}{l}25.0 \\
50.0 \\
75.0 \\
25.0 \\
50.0 \\
75.0 \\
25.0 \\
50.0 \\
75.0 \\
25.0 \\
50.0 \\
75.0 \\
25.0 \\
50.0 \\
75.0 \\
25.0 \\
50.0 \\
75.0\end{array}$ & $\begin{array}{l}26100 \\
26100 \\
26100 \\
39400 \\
39400 \\
39400 \\
52800 \\
52800 \\
52800 \\
57300 \\
57300 \\
57300 \\
40000 \\
40000 \\
40000 \\
37800 \\
37800 \\
37800\end{array}$ & $\begin{array}{r}477 \\
528 \\
545 \\
546 \\
721 \\
630 \\
419 \\
349 \\
347 \\
192 \\
191 \\
195 \\
73 \\
61 \\
68 \\
62 \\
59 \\
53\end{array}$ & $\begin{array}{r}33600 \\
37200 \\
38400 \\
58100 \\
76700 \\
67000 \\
59700 \\
49800 \\
49500 \\
29700 \\
29500 \\
30200 \\
7880 \\
6590 \\
7340 \\
6330 \\
6020 \\
5410\end{array}$ & & & \\
\hline DATE & TIME & $\begin{array}{l}\text { SAMP LE } \\
\text { LOCAT. } \\
\text { X-SECT. } \\
\text { LOOKING } \\
\text { UPSTRM. } \\
\text { (\& EROM } \\
\text { R BANK) }\end{array}$ & $\begin{array}{c}\text { BED } \\
\text { MAT. } \\
\text { SIEVE } \\
\text { DIAM. } \\
\text { FINER } \\
\text { THAN } \\
.062 \mathrm{MM}\end{array}$ & $\begin{array}{c}\text { BED } \\
\text { MAT. } \\
\text { SIEVE } \\
\text { DIAM. } \\
+ \text { FINER } \\
\text { THAN } \\
.125 \mathrm{MM}\end{array}$ & $\begin{array}{c}\text { BED } \\
\text { MAT. } \\
\text { SIEVE } \\
\text { DIAM. } \\
\text { + FINER } \\
\text { THAN } \\
.250 \mathrm{MM}\end{array}$ & $\begin{array}{c}\text { BED } \\
\text { MAT. } \\
\text { SIEVE } \\
\text { DIAM. } \\
\text { \& FINER } \\
\text { THAN } \\
.500 \mathrm{MM}\end{array}$ & $\begin{array}{c}\text { BED } \\
\text { MAT. } \\
\text { SIEVE } \\
\text { DIAM. } \\
+ \text { FINER } \\
\text { THAN } \\
1.00 \mathrm{MM}\end{array}$ & $\begin{array}{c}\text { BED } \\
\text { MAT. } \\
\text { SIEVE } \\
\text { DIAM. } \\
\text { + FINER } \\
\text { THAN } \\
2.00 \mathrm{MM}\end{array}$ & $\begin{array}{c}\text { BED } \\
\text { MAT. } \\
\text { SIEVE } \\
\text { DIAM. } \\
\text { EINER } \\
\text { THAN } \\
4.00 \mathrm{MM}\end{array}$ & $\begin{array}{c}\text { BED } \\
\text { MAT. } \\
\text { SIEVE } \\
\text { DIAM. } \\
\text { * FINER } \\
\text { THAN } \\
8.00 \mathrm{MM}\end{array}$ & $\begin{array}{c}\text { BED } \\
\text { MAT. } \\
\text { SIEVE } \\
\text { DIAM. } \\
+ \text { FINER } \\
\text { THAN } \\
16.0 \mathrm{MM}\end{array}$ \\
\hline $\begin{array}{l}\text { AN } \\
20 \ldots \\
20 \ldots \\
20 \ldots\end{array}$ & $\begin{array}{l}0815 \\
0830 \\
0845\end{array}$ & $\begin{array}{l}25.0 \\
50.0 \\
75.0\end{array}$ & $-\begin{array}{l}0 \\
0\end{array}$ & $-\begin{array}{l}0 \\
0\end{array}$ & $--_{0}^{3}$ & $--_{20}^{35}$ & $--_{58}^{39}$ & $--{ }^{41}$ & $\begin{array}{r}47 \\
--\quad 73\end{array}$ & $\begin{array}{r}-{ }^{64} \\
83\end{array}$ & $-{ }^{91}$ \\
\hline
\end{tabular}


SURFACE-WATER SITES--Cont Inued

02448000 NOXUBEE RIVER AT MACON, MS

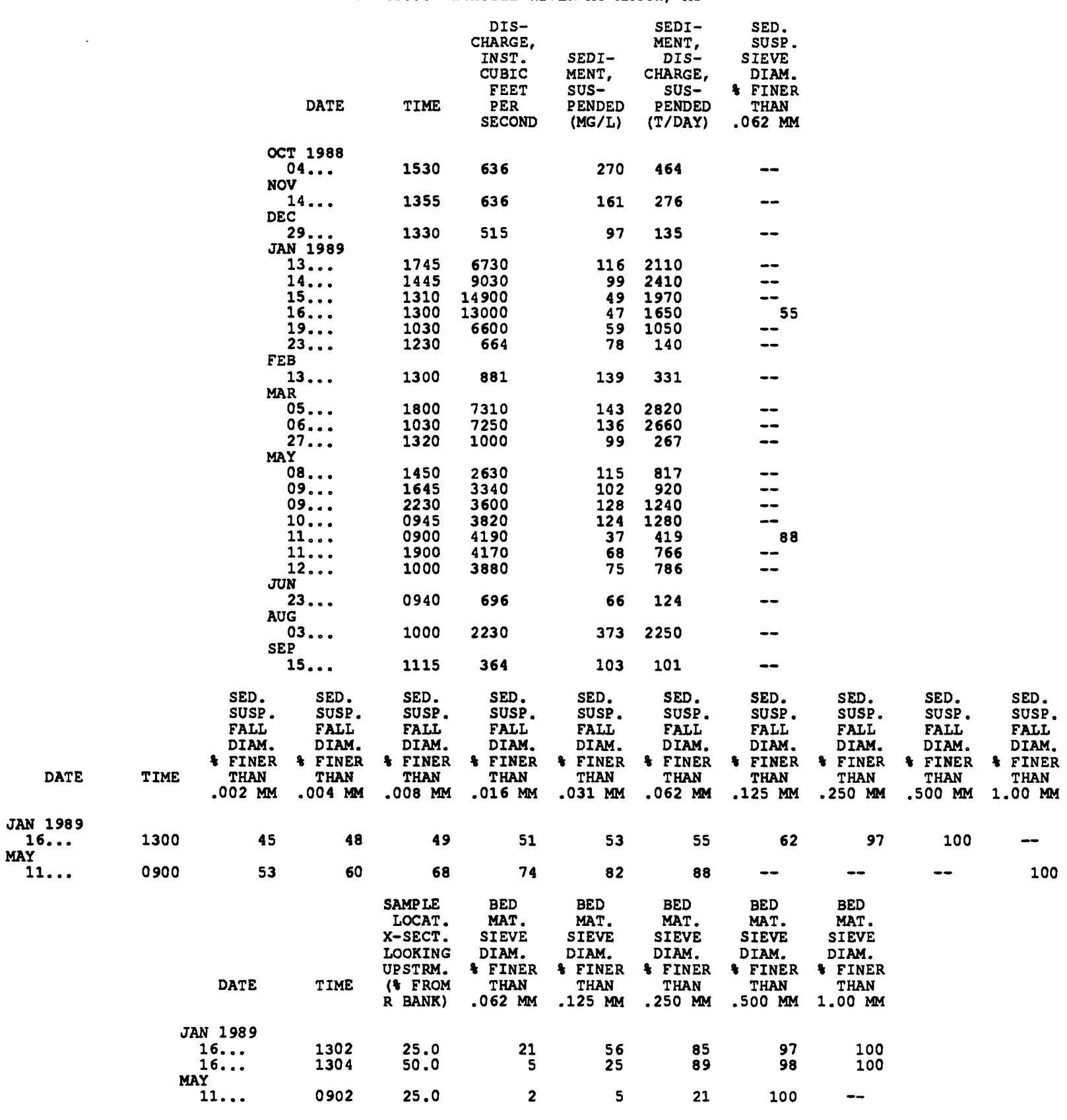


SURFACE-WATER SITES--COnt 1 nued

02448500 NOXUBEE RIVER NEAR GEIGER, AL

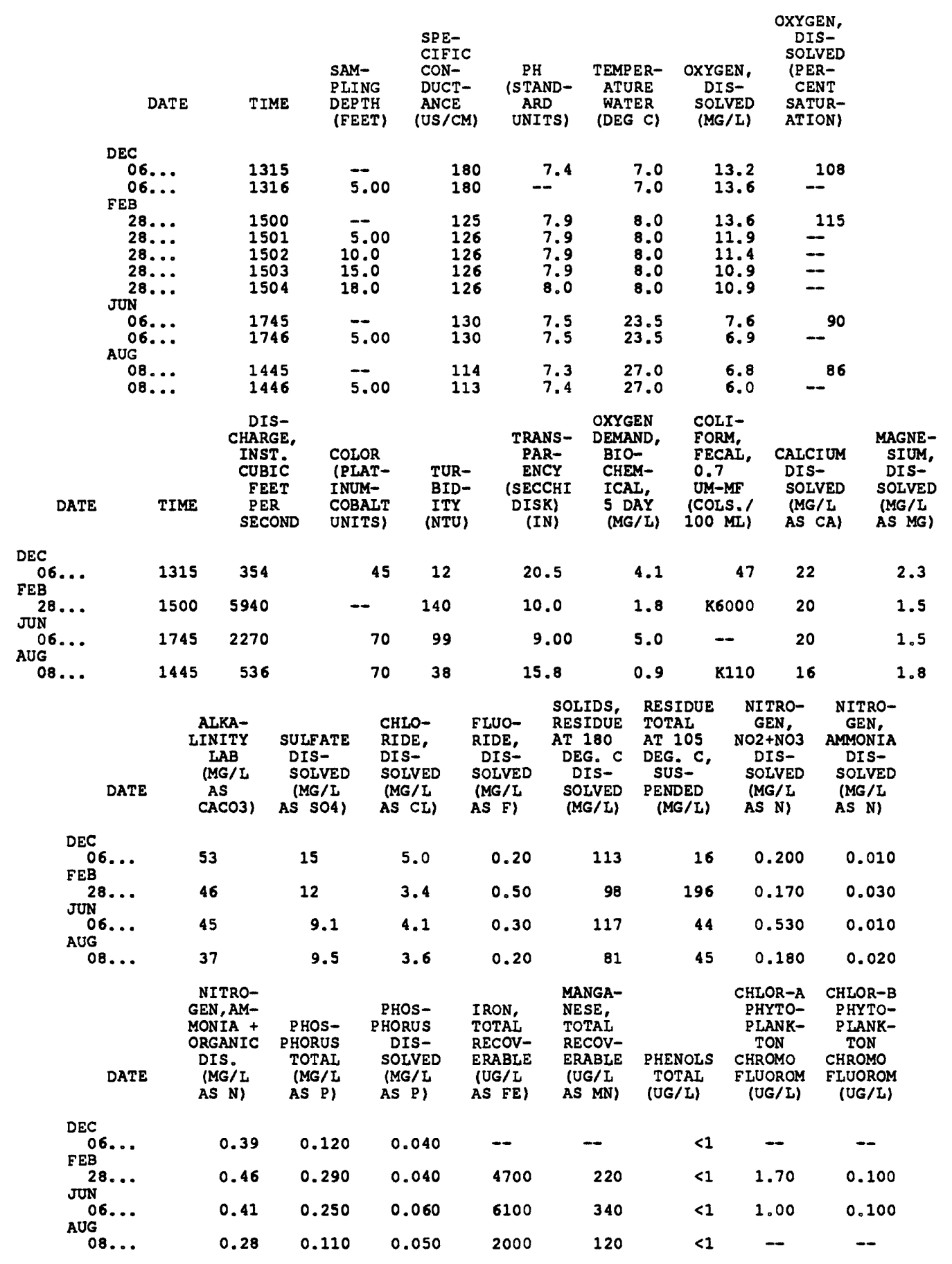


SURFACE-WATER SITES--COnt Inued

02449000 TOMBIGBEE RIVER AT GAINESVILIE, AL

\begin{tabular}{|c|c|c|c|c|c|c|c|c|c|c|c|}
\hline DATE & TIME & $\begin{array}{l}\text { DIS- } \\
\text { CHARGE, } \\
\text { INST. } \\
\text { CUBIC } \\
\text { FEET } \\
\text { PER } \\
\text { SECOND }\end{array}$ & $\begin{array}{l}\text { SPE- } \\
\text { CIFIC } \\
\text { CON- } \\
\text { DUCT- } \\
\text { ANCE } \\
\text { (US/CM) }\end{array}$ & $\begin{array}{c}\text { PH } \\
\text { (STAND- } \\
\text { ARD } \\
\text { UNITS) }\end{array}$ & $\begin{array}{c}\text { TEMPER- } \\
\text { ATURE } \\
\text { WATER } \\
\text { (DEG C) }\end{array}$ & $\begin{array}{l}\text { TUR- } \\
\text { BID- } \\
\text { ITY } \\
\text { (NTU) }\end{array}$ & $\begin{array}{c}\text { OXYGEN, } \\
\text { DIS- } \\
\text { SOIVED } \\
\text { (MG/L) }\end{array}$ & $\begin{array}{c}\text { OXYGEN, } \\
\text { DIS- } \\
\text { SOLVED } \\
\text { (PER- } \\
\text { CENT } \\
\text { SATUR- } \\
\text { ATION) }\end{array}$ & $\begin{array}{l}\text { COLI- } \\
\text { FORM, } \\
\text { FECAL, } \\
0.7 \\
\text { UM-MF } \\
\text { (COIS.' } \\
\text { IOO ML) }\end{array}$ & $\begin{array}{c}\text { STREP - } \\
\text { TOCOCCI } \\
\text { FECAL, } \\
\text { KF AGAR } \\
\text { (COIS. } \\
\text { PER } \\
100 \mathrm{ML} \text { ) }\end{array}$ & $\begin{array}{l}\text { HARD- } \\
\text { NESS } \\
\text { TOTAL } \\
\text { (MG/L } \\
\text { AS } \\
\text { CACO3) }\end{array}$ \\
\hline${ }_{\mathrm{OV}}^{\mathrm{CT}} \ldots$ & 0945 & E1000 & 121 & 7.4 & 20.0 & 16 & 9.2 & 101 & 12 & 1 & 47 \\
\hline${ }_{E C}^{16} \ldots$ & 0930 & 56000 & 150 & 7.6 & 17.0 & 8.5 & 9.5 & 99 & 130 & 120 & 56 \\
\hline JAN & 0825 & E5900 & 95 & 7.2 & 10.0 & 23 & 12.4 & 110 & 11 & 11 & 39 \\
\hline $10 \ldots$ & 1030 & E32000 & 110 & 7.3 & 9.0 & 46 & 12.2 & 105 & $>600$ & 3400 & 48 \\
\hline $\begin{array}{r}01 \ldots \\
15 \ldots \\
A P R\end{array}$ & $\begin{array}{l}1400 \\
1000\end{array}$ & $\begin{array}{l}E 74000 \\
E 14500\end{array}$ & $\begin{array}{r}102 \\
72\end{array}$ & $\begin{array}{l}7.3 \\
7.2\end{array}$ & $\begin{array}{r}8.0 \\
13.5\end{array}$ & $\begin{array}{r}140 \\
27\end{array}$ & $\begin{array}{l}11.6 \\
10.8\end{array}$ & $\begin{array}{r}98 \\
104\end{array}$ & $\begin{array}{r}4200 \\
49\end{array}$ & $\begin{array}{r}8800 \\
K 17\end{array}$ & $\begin{array}{l}44 \\
28\end{array}$ \\
\hline$\underset{\operatorname{MAY}}{17}$ & 1015 & 55400 & 79 & 7.5 & 15.0 & 17 & 10.8 & 107 & 27 & 15 & 33 \\
\hline JUN $09 .$. & 1000 & E21500 & 124 & 7.8 & 20.0 & 25 & 8.6 & 96 & 120 & 59 & 48 \\
\hline JuL & 1300 & E27600 & 127 & 7.5 & 26.0 & 42 & 8.1 & 102 & K30 & 470 & 53 \\
\hline AUG $11 \ldots$ & 1000 & $E 15400$ & 100 & 7.5 & 30.0 & 31 & 6.8 & 90 & 140 & 750 & 43 \\
\hline SER & 1215 & 21700 & 111 & 7.1 & 29.0 & 19 & 7.4 & 96 & K15 & K19 & 43 \\
\hline $13 \ldots$ & 1000 & $E 500$ & 136 & 7.4 & 29.5 & 7.0 & 7.5 & 99 & K19 & 30 & 52 \\
\hline DATE & $\begin{array}{l}\text { CALCIUM } \\
\text { DIS- } \\
\text { SOLVED } \\
\text { (MG/I } \\
\text { AS CA) }\end{array}$ & $\begin{array}{l}\text { MAGNE- } \\
\text { SIUM, } \\
\text { DIS- } \\
\text { SOLVED } \\
\text { (MG/L } \\
\text { AS MG) }\end{array}$ & $\begin{array}{l}\text { SODIUM, } \\
\text { DIS- } \\
\text { SOLVED } \\
\text { (MG/L } \\
\text { AS NA) }\end{array}$ & $\begin{array}{l}\text { SODIUM } \\
\text { PERCENT }\end{array}$ & $\begin{array}{c}\text { SODIUM } \\
\text { AD- } \\
\text { SORP- } \\
\text { TION } \\
\text { RATIO }\end{array}$ & $\begin{array}{l}\text { POTAS- } \\
\text { SIUM, } \\
\text { DIS- } \\
\text { SOIVED } \\
\text { (MG/L } \\
\text { AS K) }\end{array}$ & $\begin{array}{l}\text { BICAR- } \\
\text { BONATE } \\
\text { WATER } \\
\text { DIS IT } \\
\text { FIELD } \\
\text { MG/I AS } \\
\text { HCO3 }\end{array}$ & $\begin{array}{l}\text { ALKA- } \\
\text { IINITY } \\
\text { WAT DIS } \\
\text { TOT IT } \\
\text { FIEID } \\
\text { MG/L AS } \\
\text { CACO3 }\end{array}$ & $\begin{array}{l}\text { SULFATE } \\
\text { DIS- } \\
\text { SOLVED } \\
\text { (MG/L } \\
\text { AS SO4) }\end{array}$ & $\begin{array}{l}\text { CHLO- } \\
\text { RIDE, } \\
\text { DIS- } \\
\text { SOLVED } \\
\text { (MG/L } \\
\text { AS CL) }\end{array}$ & $\begin{array}{l}\text { FLUO- } \\
\text { RIDE, } \\
\text { DIS- } \\
\text { SOLVED } \\
\text { (MG/I } \\
\text { AS F) }\end{array}$ \\
\hline OCT & & & & & & & & & & & \\
\hline${ }_{\text {Nov }}^{12 \ldots}$ & 16 & 1.7 & 5.4 & 19 & 0.3 & 2.5 & 43 & 35 & 15 & 7.2 & 0.10 \\
\hline${ }_{D E C}^{16} \ldots$ & 1.9 & 2.0 & 5.7 & 17 & 0.3 & 2.6 & 44 & 36 & 16 & 11 & 0.10 \\
\hline JAN $07 \ldots$ & 13 & 1.6 & 3.2 & 14 & 0.2 & 2.3 & 34 & 28 & 16 & 4.7 & $<0.10$ \\
\hline $\operatorname{MAR}^{10} \ldots$ & 17 & 1.4 & 3.2 & 12 & 0.2 & 2.0 & 41 & 34 & 17 & 5.7 & 0.10 \\
\hline $\begin{array}{l}01 \ldots \\
15 \ldots \\
A P R\end{array}$ & $\begin{array}{l}16 \\
9.3\end{array}$ & $\begin{array}{l}1.0 \\
1.2\end{array}$ & $\begin{array}{l}2.3 \\
2.3\end{array}$ & $\begin{array}{l}10 \\
14\end{array}$ & $\begin{array}{l}0.2 \\
0.2\end{array}$ & $\begin{array}{l}1.5 \\
1.3\end{array}$ & $\begin{array}{l}38 \\
25\end{array}$ & $\begin{array}{l}31 \\
20\end{array}$ & $\begin{array}{l}14 \\
13\end{array}$ & $\begin{array}{l}3.8 \\
3.5\end{array}$ & $\begin{array}{l}0.10 \\
0.10\end{array}$ \\
\hline $\operatorname{MAY}^{17 \ldots}$ & 11 & 1.4 & 2.5 & 13 & 0.2 & 1.3 & 27 & 22 & 7.7 & 3.3 & 0.10 \\
\hline$\underset{\text { JUN }}{09 . . .}$ & 17 & 1.4 & 3.7 & 14 & 0.2 & 1.9 & 46 & 37 & 10 & 5.4 & 0.10 \\
\hline JUI & 19 & 1.4 & 3.8 & 13 & 0.2 & 1.9 & 50 & 41 & 9.0 & 6.7 & 0.10 \\
\hline AUG $11 \ldots$ & 15 & 1.3 & 2.7 & 12 & 0.2 & 1.7 & 46 & 38 & 6.0 & 3.0 & 0.10 \\
\hline $\begin{array}{l}09 . . . \\
\text { SEP }\end{array}$ & 15 & 1.4 & 3.0 & 13 & 0.2 & 1.7 & 45 & 37 & 7.0 & 5.3 & 0.10 \\
\hline $13 \ldots$ & 18 & 1.6 & 4.9 & 16 & 0.3 & 2.0 & 51 & 42 & 9.0 & 8.6 & 0.10 \\
\hline
\end{tabular}


SURFACE-WATER SITES--Cont1nued

02449000 TOMBIGBEE RIVER AT GAINESVILLE, AL--Cont1nued

DATE

$\begin{array}{ccc} & \text { SOLIDS, } & \text { SOL } \\ \text { SIIICA, } & \text { RESIDUE } & \text { SUM } \\ \text { DIS- } & \text { AT } 180 & \text { CO } \\ \text { SOLVED } & \text { DEG.C } & \text { TO } \\ \text { (MG/L } & \text { DIS- } \\ \text { AS } & \text { SOLVED } & \\ \text { SIO2) } & \text { (MG/L) } & \end{array}$

$\begin{array}{lc}\text { SOLIDS, } & \\ \text { SUM OF } & \text { SOLIDS, } \\ \text { CONSTI- } & \text { DIS- } \\ \text { TUENTS, } & \text { SOLVED } \\ \text { DIS- } & \text { (TONS } \\ \text { SOLVED } & \text { PER } \\ \text { (MG/I) } & \text { AC-FT) }\end{array}$

NITRO-

NITRO- NITRO-

GEN, GEN, GEN, AM-

NO2+NO3

ONIA MONIA +

SOLVED

(MG/L
AS N)

DOLVED TOTAT PHORS

(MG/L (MG/L (MG/L

$\begin{array}{ll}\text { (MG/L } & \text { (MG/L } \\ \text { AS N) AS P) }\end{array}$

PHOS-

PHORUS

ORTHO

DIS- DIS-

(MG/I

(MG/L (MG/L
AS P) AS P)

\begin{tabular}{|c|c|c|c|c|c|c|c|c|c|c|}
\hline $\begin{array}{l}\infty \mathrm{CT} \\
12 \ldots \\
\text { Nov }\end{array}$ & 5.9 & 90 & 76 & 0.12 & 0.230 & 0.040 & 0.70 & 0.080 & 0.040 & 0.020 \\
\hline EC $16 \ldots$ & 4.6 & 84 & 83 & 0.11 & 0.160 & 0.020 & 0.70 & 0.070 & 0.030 & 0.010 \\
\hline $07 \ldots$ & 7.0 & 78 & 65 & 0.11 & $<0.100$ & 0.050 & 0.60 & 0.080 & 0.030 & 0.010 \\
\hline $10 \ldots$ & 7.3 & 112 & 74 & 0.15 & 0.130 & 0.090 & 0.80 & 0.120 & 0.070 & 0.050 \\
\hline $\begin{array}{r}01 \ldots \\
15 \ldots \\
A P R\end{array}$ & $\begin{array}{l}5.2 \\
5.5\end{array}$ & $\begin{array}{l}79 \\
53\end{array}$ & $\begin{array}{l}64 \\
49\end{array}$ & $\begin{array}{l}0.11 \\
0.07\end{array}$ & $\begin{array}{r}0.180 \\
<0.100\end{array}$ & $\begin{array}{l}0.060 \\
0.020\end{array}$ & $\begin{array}{l}0.90 \\
0.40\end{array}$ & $\begin{array}{l}0.250 \\
0.040\end{array}$ & $\begin{array}{l}0.030 \\
0.010\end{array}$ & $\begin{array}{l}0.020 \\
0.020\end{array}$ \\
\hline $\operatorname{MAY}^{17} \ldots$ & 6.6 & 62 & 48 & 0.08 & $<0.100$ & 0.030 & 0.30 & 0.060 & 0.020 & $<0.010$ \\
\hline$\underset{\pi N}{09 . . .}$ & 5.6 & 139 & 68 & 0.19 & $<0.100$ & $<0.010$ & 0.70 & 0.110 & -- & $<0.010$ \\
\hline$\underset{\pi}{07} . .$. & 5.9 & 77 & 74 & 0.10 & 0.350 & 0.060 & 0.90 & 0.080 & 0.040 & 0.030 \\
\hline${ }_{\text {AUG }}^{11 \ldots .}$ & 7.6 & 67 & 61 & 0.09 & 0.110 & 0.040 & 0.40 & 0.070 & 0.040 & 0.030 \\
\hline EP $09 .$. & 6.9 & 73 & 64 & 0.10 & 0.140 & 0.060 & 0.40 & 0.060 & 0.020 & 0.020 \\
\hline $13 \ldots$ & 4.4 & 90 & 74 & 0.12 & $<0.100$ & 0.030 & 0.30 & 0.050 & 0.010 & $<0.010$ \\
\hline DATE & $\begin{array}{l}\text { ALUM- } \\
\text { INUM, } \\
\text { DIS- } \\
\text { SOLVED } \\
\text { (UG/I } \\
\text { AS AL) }\end{array}$ & $\begin{array}{c}\text { ARSENIC } \\
\text { DIS- } \\
\text { SOLVED } \\
\text { (UG/I } \\
\text { AS AS) }\end{array}$ & $\begin{array}{l}\text { BARIUM, } \\
\text { DIS- } \\
\text { SOLVED } \\
\text { (UG/I } \\
\text { AS BA) }\end{array}$ & $\begin{array}{l}\text { BERYL- } \\
\text { LIUM, } \\
\text { DIS- } \\
\text { SOLVED } \\
\text { (UG/L } \\
\text { AS BE) }\end{array}$ & $\begin{array}{c}\text { CADMIUM } \\
\text { DIS- } \\
\text { SOLVED } \\
\text { (UG/L } \\
\text { AS CD) }\end{array}$ & $\begin{array}{l}\text { CHRO- } \\
\text { MIUM, } \\
\text { DIS- } \\
\text { SOLVED } \\
\text { (UG/I } \\
\text { AS CR) }\end{array}$ & $\begin{array}{l}\text { COBALT, } \\
\text { DIS- } \\
\text { SOLVED } \\
\text { (UG/I } \\
\text { AS CO) }\end{array}$ & $\begin{array}{l}\text { COPPER, } \\
\text { DIS- } \\
\text { SOLVED } \\
\text { (UG/L } \\
\text { AS CU) }\end{array}$ & $\begin{array}{l}\text { IRON, } \\
\text { DIS- } \\
\text { SOLVED } \\
\text { (UG/L } \\
\text { AS FE) }\end{array}$ & $\begin{array}{l}\text { LEAD, } \\
\text { DIS- } \\
\text { SOLVED } \\
\text { (UG/L } \\
\text { AS PB) }\end{array}$ \\
\hline
\end{tabular}

\begin{tabular}{|c|c|c|c|c|c|c|c|c|c|c|}
\hline $\begin{array}{l}\text { OCT } \\
12 \ldots \\
M A R\end{array}$ & 30 & 1 & 24 & 0.9 & $<1.0$ & 1 & $<3$ & 2 & 110 & $<5$ \\
\hline $\begin{array}{c}\text { MAR } \\
01 \ldots \\
A P R\end{array}$ & 100 & $<1$ & 20 & $<0.5$ & 3.0 & $<1$ & $<3$ & 15 & 180 & $<5$ \\
\hline $\mathrm{UG}^{17 \ldots}$ & 100 & $<1$ & 30 & $<0.5$ & $<1.0$ & $<1$ & $<3$ & 2 & 470 & $<5$ \\
\hline $09 \ldots$ & 10 & $<1$ & 21 & $<0.5$ & $<1.0$ & $<1$ & $<3$ & 1 & 140 & $<1$ \\
\hline DATE & $\begin{array}{c}\text { LITHIUM } \\
\text { DIS- } \\
\text { SOIVED } \\
\text { (UG/L } \\
\text { AS II) }\end{array}$ & $\begin{array}{l}\text { MANGA- } \\
\text { NESE, } \\
\text { DIS- } \\
\text { SOIVED } \\
\text { (UG/L } \\
\text { AS MN) }\end{array}$ & $\begin{array}{c}\text { MERCURY } \\
\text { DIS- } \\
\text { SOLVED } \\
\text { (UG/I } \\
\text { AS HG) }\end{array}$ & $\begin{array}{l}\text { MOLYB- } \\
\text { DENUM, } \\
\text { DIS- } \\
\text { SOLVED } \\
\text { (UG/L } \\
\text { AS MO) }\end{array}$ & $\begin{array}{l}\text { NICREL, } \\
\text { DIS- } \\
\text { SOLVED } \\
\text { (UG/L } \\
\text { AS NI) }\end{array}$ & $\begin{array}{l}\text { SEIE- } \\
\text { NIUM, } \\
\text { DIS- } \\
\text { SOLVED } \\
\text { (UG/L } \\
\text { AS SE) }\end{array}$ & $\begin{array}{c}\text { SILVER, } \\
\text { DIS- } \\
\text { SOLVED } \\
\text { (UG/I } \\
\text { AS AG) }\end{array}$ & $\begin{array}{l}\text { STRON- } \\
\text { TIUM, } \\
\text { DIS- } \\
\text { SOLVED } \\
\text { (UG/L } \\
\text { AS SR) }\end{array}$ & $\begin{array}{l}\text { VANA- } \\
\text { DIUM, } \\
\text { DIS- } \\
\text { SOLVED } \\
\text { (UG/I } \\
\text { AS V) }\end{array}$ & $\begin{array}{l}\text { ZINC, } \\
\text { DIS- } \\
\text { SOIVED } \\
\text { (UG/L } \\
\text { AS } \mathrm{ZN} \text { ) }\end{array}$ \\
\hline
\end{tabular}
OCT

\begin{tabular}{|c|c|c|c|c|c|c|c|c|c|c|}
\hline $\begin{array}{l}\mathrm{OCT} \\
12 \ldots\end{array}$ & $<4$ & $<1$ & $<0.1$ & $<10$ & 2 & $<1$ & $<1.0$ & 100 & $<6$ & 11 \\
\hline $\begin{array}{l}01 \ldots \\
A P R\end{array}$ & $<4$ & 41 & 0.1 & $<10$ & 3 & $<1$ & $<1.0$ & 100 & $<6$ & 23 \\
\hline AUG $17 \ldots$ & $<4$ & 45 & $<0.1$ & $<10$ & 5 & $<1$ & $<1.0$ & 72 & $<6$ & 12 \\
\hline 09. & $<4$ & 3 & 0.4 & $<10$ & 1 & $<1$ & $<1,0$ & 100 & $<6$ & \\
\hline
\end{tabular}


SURFACE-WATER SITES--Cont1 nued

02449000 TOMBIGBEE RIVER AT GAINESVILLE, AL--Cont1nued

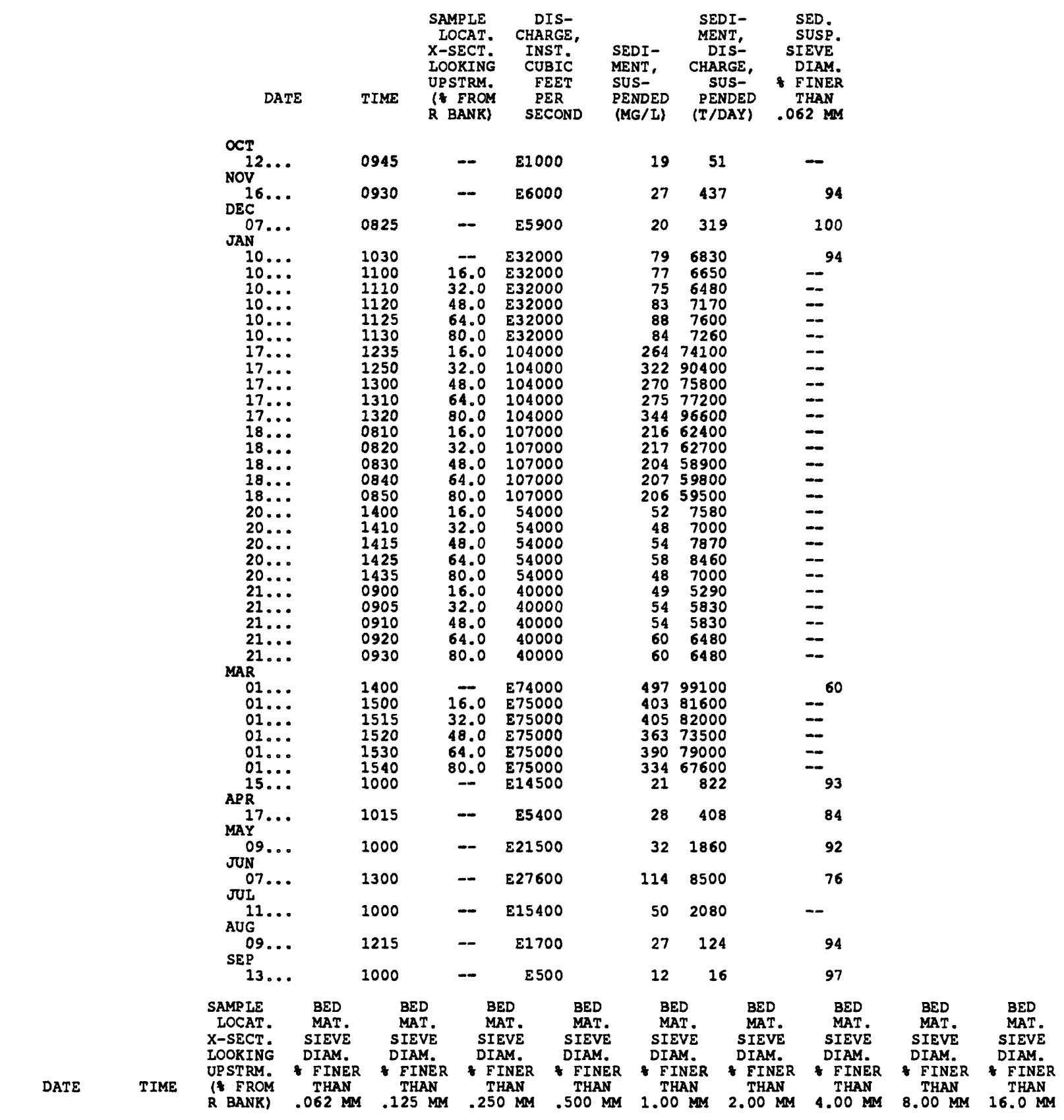

$\begin{array}{ccc}\text { JAN } & & \\ 10 \ldots & 1200 & 25.0 \\ 10 \ldots . . & 1210 & 50.0 \\ 10 \ldots & 1220 & 75.0 \\ 21 \ldots . . & 1000 & 25.0 \\ 21 \ldots & 1015 & 50.0 \\ 21 \ldots & 1030 & 75.0\end{array}$

$\begin{array}{lr}25.0 & 0 \\ 50.0 & 0 \\ 75.0 & <1 \\ 25.0 & 0 \\ 50.0 & 0 \\ 75.0 & 0\end{array}$

$\begin{array}{rr}<1 & 3 \\ <1 & 3 \\ <1 & 16 \\ 0 & 5 \\ 0 & 7 \\ 1 & 19\end{array}$

$\begin{array}{rr}14 & 16 \\ 28 & 42 \\ 98 & 100 \\ 14 & 16 \\ 17 & 18 \\ 89 & 99\end{array}$

20
45
100
17
20
100

28
52
100
20
29
100

47
71
100
29
67
100

93
95
100
75
98
100 
SUREACE-WATER SITES--Cont1nued

02449000 TOMBIGBEE RIVER AT GAINESVILLE, AL--Cont1nued

SPECIFIC CONDUCTANCE, MICROSIEMENS PER CENTIMETER AT 25 DEG. C, WATER YEAR OCTOBER 1988 TO SEPTEMBER 1989

DAY

MAX MIN MEAN
OCTOBER

1
2
3
4
5
6
7
8
9
10
11
12
13
14
15
16
17
18
19
20
21
22
23
24
25
26
27
28
29
31
31

$\begin{array}{ll}187 & 16 \\ 177 & 14 \\ 156 & 14 \\ 160 & 145 \\ 155 & 146\end{array}$

164
148
143
145
146

174
169
147
154
150

$167 \quad 153 \quad 159$

$\begin{array}{lll}166 & 150 & 159 \\ 161 & 155 & 160\end{array}$

$\begin{array}{lll}161 & 155 & 160 \\ 156 & 151 & 154 \\ 157 & 151 & 155\end{array}$

$156 \quad 147 \quad 151$

$\begin{array}{lll}156 & 147 & 151 \\ 149 & 121 & 132 \\ 126 & 120 & 123\end{array}$

$\begin{array}{lll}126 & 120 & 123 \\ 124 & 120 & 122\end{array}$

$\begin{array}{lll}124 & 120 & 122 \\ 122 & 118 & 120\end{array}$

$\begin{array}{lll}124 & 119 & 122 \\ 121 & 117 & 119\end{array}$

$\begin{array}{lll}121 & 117 & 119 \\ 124 & 115 & 118\end{array}$

$\begin{array}{lll}124 & 115 & 118\end{array}$

$123 \quad 115 \quad 119$

$\begin{array}{lll}153 & 115 & 131 \\ 166 & 131 & 147 \\ 152 & 120 & 138 \\ 137 & 114 & 122\end{array}$

$\begin{array}{lll}137 & 114 & 122 \\ 159 & 133 & 144\end{array}$

$144 \quad 119 \quad 130$

127

133
115

115

$\begin{array}{ll}117 & 120 \\ 110 & 120 \\ 106 & 110 \\ 104 & 109\end{array}$

$\begin{array}{lll}118 & 101 & 109\end{array}$

MONTH

$\begin{array}{lll}187 & 101 & 136\end{array}$
MAX MIN MEAN NOVEMBER

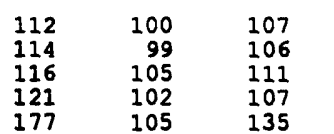

$177 \quad 105 \quad 135$

$\begin{array}{lll}190 & 144 & 163 \\ 158 & 123 & 137 \\ 143 & 122 & 130 \\ 144 & 128 & 136 \\ 143 & 128 & 133\end{array}$

$\begin{array}{lll}138 & 129 & 133 \\ 144 & 134 & 137\end{array}$

189
181

160

165
176

176
180
145

145
152

150
131
149
148

148
138

125
141
124

$\begin{array}{r}99 \\ 111 \\ \hline\end{array}$

190

153
157

140
135

$\begin{array}{ll}143 & 154 \\ 140 & 148\end{array}$

$\begin{array}{ll}140 & 148 \\ 144 & 149\end{array}$

$\begin{array}{ll}127 & 138 \\ 141 & 146\end{array}$

$\begin{array}{ll}132 & 144 \\ 126 & 128\end{array}$

$\begin{array}{ll}131 & 128 \\ 138 & 141\end{array}$

$138 \quad 144$

$125 \quad 131$

$111 \quad 119$

\begin{tabular}{rr}
114 & 119 \\
98 & 124 \\
94 & 107 \\
98 & 96 \\
\hline
\end{tabular}

$\begin{array}{rr}98 & 96 \\ -- & 105 \\ -\end{array}$

$94 \quad 132$

FEBRUARY

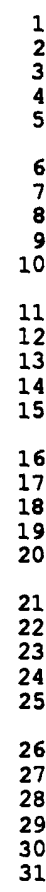

$\begin{array}{rr}95 & 99 \\ 100 & 103 \\ 109 & 117 \\ 118 & 121 \\ 115 & 122\end{array}$

$$
\begin{aligned}
& 103 \\
& 109 \\
& 127 \\
& 123 \\
& 129
\end{aligned}
$$

$119 \quad 113 \quad 116$

$\begin{array}{lll}119 & 113 & 116 \\ 123 & 116 & 120\end{array}$

$119 \quad 114 \quad 116$

$\begin{array}{lll}117 & 112 & 114 \\ 119 & 106 & 112\end{array}$

11

$112 \quad 104 \quad 108$

$\begin{array}{lll}112 & 104 & 108 \\ 103 & 100 & 102 \\ 119 & 103 & 107\end{array}$

$\begin{array}{rrr}119 & 103 & 107 \\ 100 & 87 & 101\end{array}$

16

$\begin{array}{rrr}101 & 90 & 94 \\ 104 & 99 & 101 \\ 114 & 100 & 106 \\ 1123 & 115 & 121\end{array}$

$\begin{array}{lll}123 & 115 & 121 \\ 134 & 120 & 127\end{array}$

$152 \quad 132 \quad 141$

$138 \quad 120 \quad 129$

$\begin{array}{rrr}118 & 101 & 111 \\ 100 & 91 & 96 \\ 91 & 87 & 89\end{array}$

$\begin{array}{lll}88 & 86 & 87\end{array}$

$\begin{array}{rrr}88 & 86 & 87 \\ 87 & 85 & 86 \\ 99 & 83 & 89 \\ --- & -- & -- \\ --- & -- & -- \\ --- & -- & --\end{array}$

MONTH

152

$83 \quad 108$

\begin{tabular}{|c|c|c|}
\hline \multicolumn{3}{|c|}{ MARCH } \\
\hline $\begin{array}{r}110 \\
96 \\
80 \\
77 \\
89\end{array}$ & $\begin{array}{l}91 \\
80 \\
66 \\
66 \\
78\end{array}$ & $\begin{array}{r}101 \\
89 \\
73 \\
69 \\
81\end{array}$ \\
\hline $\begin{array}{l}97 \\
89 \\
82 \\
76 \\
74\end{array}$ & $\begin{array}{l}79 \\
82 \\
76 \\
68 \\
69\end{array}$ & $\begin{array}{l}86 \\
86 \\
79 \\
72 \\
70\end{array}$ \\
\hline $\begin{array}{l}80 \\
82 \\
82 \\
80 \\
81\end{array}$ & $\begin{array}{l}73 \\
77 \\
74 \\
72 \\
72\end{array}$ & $\begin{array}{l}77 \\
79 \\
78 \\
76 \\
76\end{array}$ \\
\hline $\begin{array}{r}88 \\
85 \\
87 \\
98 \\
104\end{array}$ & $\begin{array}{l}76 \\
78 \\
78 \\
77 \\
77\end{array}$ & $\begin{array}{l}80 \\
81 \\
82 \\
85 \\
88\end{array}$ \\
\hline $\begin{array}{l}122 \\
109 \\
110 \\
128 \\
128\end{array}$ & $\begin{array}{r}75 \\
101 \\
106 \\
107 \\
122\end{array}$ & $\begin{array}{l}102 \\
106 \\
108 \\
115 \\
125\end{array}$ \\
\hline $\begin{array}{l}122 \\
121 \\
131 \\
138 \\
129 \\
115\end{array}$ & $\begin{array}{l}117 \\
111 \\
106 \\
104 \\
112 \\
107\end{array}$ & $\begin{array}{l}119 \\
117 \\
114 \\
112 \\
118 \\
109\end{array}$ \\
\hline 138 & 66 & 92 \\
\hline
\end{tabular}

MAX

MIN MEAN

$\begin{array}{rrr}112 & 108 & 110 \\ 111 & 104 & 107 \\ 106 & 101 & 104 \\ 109 & 96 & 101 \\ 100 & 94 & 97\end{array}$

$123 \quad 91$

$\begin{array}{rrr}123 & 91 & 99 \\ 116 & 88 & 94 \\ 99 & 84 & 91 \\ 124 & 79 & 90 \\ 131 & 81 & 91\end{array}$

$\begin{array}{rll}96 & 80 & 89 \\ 112 & 81 & 88 \\ 130 & 80 & 93 \\ 116 & 83 & 92 \\ 119 & 81 & 91\end{array}$

$\begin{array}{lll}107 & 82 & 90\end{array}$

$\begin{array}{lll}107 & 82 & 90 \\ 131 & 85 & 97 \\ 133 & 87 & 94 \\ 130 & 88 & 98\end{array}$

$\begin{array}{lll}130 & 88 & 98 \\ 118 & 84 & 90\end{array}$

112
96

162
119

84
89
88
106

88
111

94
93
109

$124 \quad 111 \quad 116$

$\begin{array}{lll}133 & 113 & 121 \\ 130 & 118 & 127\end{array}$

136

152

152

128

133
140

140
139

162

79

131
141

145

104

APRIL

$\begin{array}{lll}118 & 106 & 110 \\ 122 & 118 & 120 \\ 126 & 114 & 119 \\ 117 & 103 & 111 \\ 125 & 103 & 113\end{array}$

$\begin{array}{rrr}121 & 109 & 116 \\ 109 & 98 & 103 \\ 98 & 89 & 92 \\ 105 & 87 & 96 \\ 98 & 91 & 96\end{array}$

98

$\begin{array}{ll}93 & 89 \\ 92 & 89 \\ 99 & 90\end{array}$

$\begin{array}{ll}98 & 80 \\ 88 & 81\end{array}$

$\begin{array}{rr}89 & 81 \\ 104 & 78 \\ 99 & 76 \\ 104 & 78 \\ 128 & 77\end{array}$

116
100
115

115
128

128
114

104
119
112

125

112

$\begin{array}{lll}128 & 76 & 97\end{array}$
MAX MIN MEAN JANUARY

$\begin{array}{rrr}143 & 126 & 136 \\ 125 & 114 & 119 \\ 113 & 108 & 110 \\ 109 & 99 & 104 \\ 101 & 94 & 98\end{array}$

$\begin{array}{rrr}96 & 92 & 94 \\ 97 & 90 & 94 \\ 94 & 78 & 85 \\ 102 & 79 & 85 \\ 114 & 99 & 108\end{array}$

$116 \quad 110 \quad 113$

$121 \quad 109 \quad 115$

$\begin{array}{rrr}113 & 106 & 110 \\ 109 & 93 & 101 \\ 93 & 80 & 87\end{array}$

$80 \quad 71 \quad 76$

$\begin{array}{lll}72 & 67 & 69 \\ 67 & 64 & 66 \\ 66 & 63 & 64 \\ 70 & 65 & 67\end{array}$

$\begin{array}{lll}82 & 69 & 75 \\ 92 & 81 & 86\end{array}$

$\begin{array}{lll}92 & 81 & 86 \\ 93 & 69 & 84 \\ 79 & 70 & 75\end{array}$

78
86 $\quad 73$

$\begin{array}{lll}86 & 73 & 78 \\ 86 & 69 & 75\end{array}$

$\begin{array}{rrr}90 & 73 & 78 \\ 90 & 72 & 80\end{array}$

$\begin{array}{rrr}113 & 94 & 92 \\ 108 & 97 & 101\end{array}$

$143 \quad 63 \quad 90$

$\begin{array}{rrrrr}89 & 91 & 111 & 101 & 106 \\ 89 & 91 & 101 & 97 & 98 \\ 90 & 95 & 99 & 97 & 98 \\ 80 & 91 & 120 & 95 & 100 \\ 81 & 85 & 119 & 106 & 110 \\ 81 & 86 & 120 & 112 & 116 \\ 78 & 86 & 112 & 102 & 107 \\ 76 & 89 & 106 & 98 & 102 \\ 78 & 91 & 125 & 106 & 116 \\ 77 & 91 & 122 & 106 & 111 \\ 82 & 87 & 127 & 117 & 120 \\ 84 & 91 & 124 & 116 & 121 \\ 85 & 91 & 142 & 123 & 132 \\ 84 & 96 & 153 & 135 & 142 \\ 83 & 94 & 148 & 131 & 137 \\ 85 & 96 & 156 & 139 & 147 \\ 85 & 97 & 165 & 146 & 157 \\ 87 & 96 & 174 & 149 & 163 \\ 91 & 103 & 166 & 143 & 155 \\ 91 & 98 & 171 & 143 & 154 \\ --5 & -9- & 165 & 136 & 150\end{array}$

$\begin{array}{lll}174 & 91 & 121\end{array}$ 
SURFACE-WATER SITES--Continued

02449000 TOMBIGBEE RIVER AT GAINESVILIE, AL--Cont1nued

SPECIFIC CONDUCTANCE, MICROSIEMENS PER CENTIMETER AT 25 DEG. C, WATER YEAR OCTOBER 1988 TO SEPTEMBER 1989

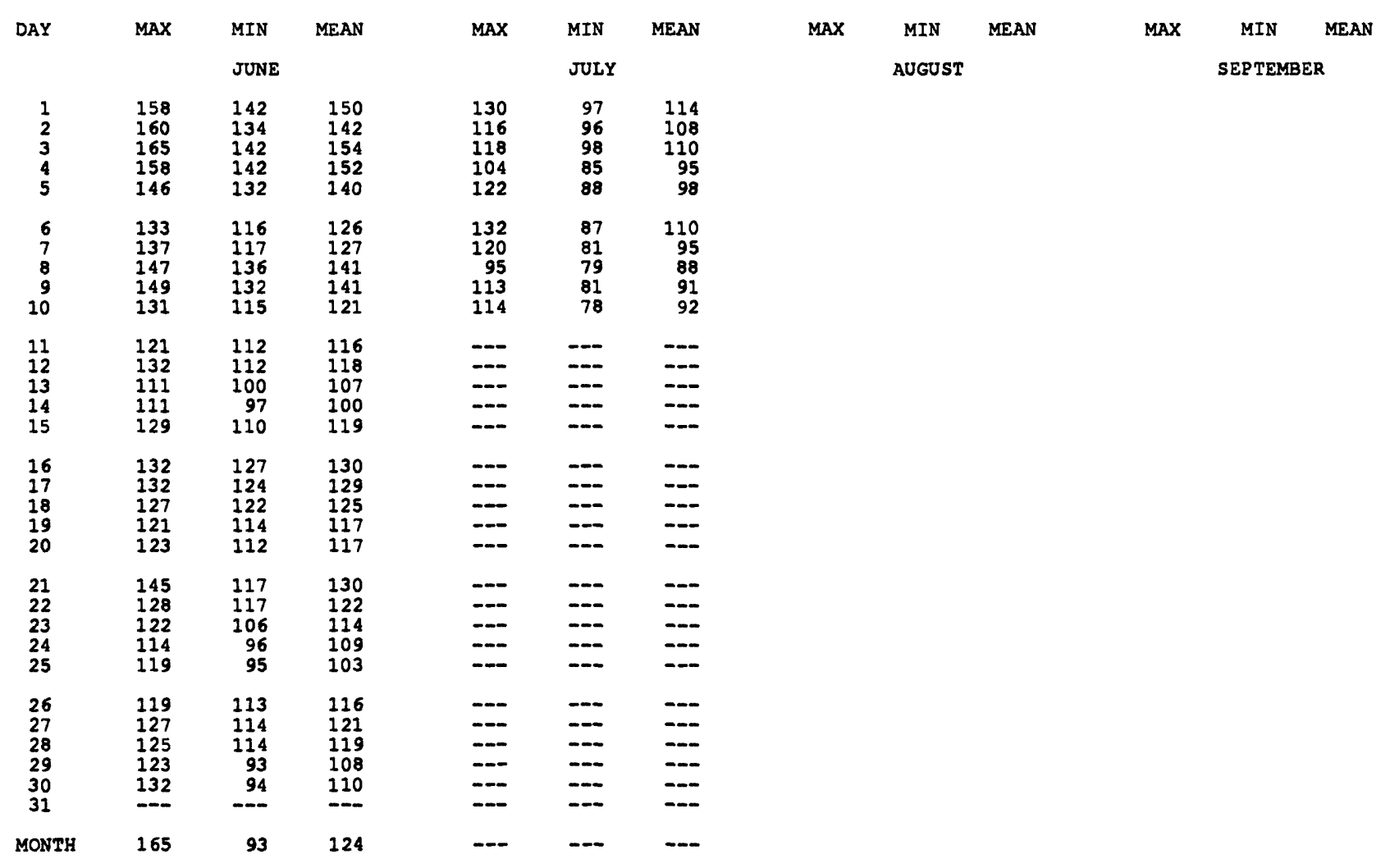

TEMPERATURE, WATER (DEG. C), WATER YEAR OCTOBER 1988 TO SEPTEMBER 1989

\begin{tabular}{|c|c|c|c|c|c|c|c|c|c|c|c|c|}
\hline \multirow[t]{2}{*}{ DAY } & $\operatorname{MAX}$ & MIN & MEAN & $\operatorname{MAX}$ & MIN & MEAN & $\operatorname{MAX}$ & MIN & MEAN & $\operatorname{MAX}$ & MIN & MEAN \\
\hline & \multicolumn{3}{|c|}{ OCTOBER } & \multicolumn{3}{|c|}{ NOVEMBER } & \multicolumn{3}{|c|}{ DECEMBER } & \multicolumn{3}{|c|}{ JANUARY } \\
\hline $\begin{array}{l}1 \\
2 \\
3 \\
4 \\
5\end{array}$ & $\begin{array}{l}21.0 \\
25.0 \\
25.0 \\
24.0 \\
23.0\end{array}$ & $\begin{array}{l}19.5 \\
22.5 \\
22.0 \\
23.5 \\
22.5\end{array}$ & $\begin{array}{l}20.5 \\
24.5 \\
23.5 \\
23.5 \\
23.0\end{array}$ & $\begin{array}{l}15.5 \\
16.5 \\
17.5 \\
18.5 \\
18.0\end{array}$ & $\begin{array}{l}.5 \\
.5 \\
.5 \\
.5 \\
.5\end{array}$ & $\begin{array}{r}8.5 \\
3.5 \\
5.5 \\
16.0 \\
10.0\end{array}$ & $\begin{array}{l}12.5 \\
12.0 \\
11.5 \\
11.0 \\
11.0\end{array}$ & $\begin{array}{l}12.0 \\
11.5 \\
11.0 \\
10.5 \\
10.5\end{array}$ & $\begin{array}{l}12.5 \\
12.0 \\
11.5 \\
11.0 \\
10.5\end{array}$ & $\begin{array}{l}11.0 \\
10.0 \\
10.0 \\
10.0 \\
10.0\end{array}$ & $\begin{array}{r}10.0 \\
9.5 \\
10.0 \\
10.0 \\
9.5\end{array}$ & $\begin{array}{l}10.5 \\
10.0 \\
10.0 \\
10.0 \\
10.0\end{array}$ \\
\hline $\begin{array}{r}6 \\
7 \\
8 \\
9 \\
10\end{array}$ & $\begin{array}{l}22.5 \\
22.0 \\
21.5 \\
21.0 \\
21.0\end{array}$ & $\begin{array}{l}21.5 \\
20.5 \\
20.0 \\
20.5 \\
20.0\end{array}$ & $\begin{array}{l}22.0 \\
21.5 \\
21.0 \\
20.5 \\
20.5\end{array}$ & $\begin{array}{l}15.5 \\
17.5 \\
16.5 \\
17.0 \\
17.0\end{array}$ & $\begin{array}{r}.5 \\
.5 \\
13.0 \\
13.0 \\
16.0\end{array}$ & $\begin{array}{r}1.5 \\
15.5 \\
14.0 \\
15.0 \\
17.0\end{array}$ & $\begin{array}{l}10.5 \\
11.0 \\
10.5 \\
10.5 \\
10.0\end{array}$ & $\begin{array}{r}9.5 \\
10.0 \\
10.0 \\
9.5 \\
9.5\end{array}$ & $\begin{array}{l}10.0 \\
10.5 \\
10.5 \\
10.0 \\
10.0\end{array}$ & $\begin{array}{l}10.5 \\
11.0 \\
11.0 \\
11.0 \\
10.5\end{array}$ & $\begin{array}{l}10.0 \\
10.0 \\
11.0 \\
10.5 \\
10.0\end{array}$ & $\begin{array}{l}10.0 \\
10.5 \\
11.0 \\
11.0 \\
10.5\end{array}$ \\
\hline $\begin{array}{l}11 \\
12 \\
13 \\
14 \\
15\end{array}$ & $\begin{array}{l}20.5 \\
20.5 \\
20.0 \\
19.5 \\
19.5\end{array}$ & $\begin{array}{l}17.5 \\
19.0 \\
19.0 \\
18.5 \\
18.0\end{array}$ & $\begin{array}{l}20.0 \\
20.0 \\
19.5 \\
19.5 \\
19.0\end{array}$ & $\begin{array}{l}17.5 \\
17.0 \\
17.0 \\
16.5 \\
17.0\end{array}$ & $\begin{array}{l}14.0 \\
16.5 \\
15.5 \\
15.5 \\
16.5\end{array}$ & $\begin{array}{l}15.5 \\
17.0 \\
16.5 \\
16.0 \\
16.5\end{array}$ & $\begin{array}{l}9.5 \\
9.5 \\
9.5 \\
9.5 \\
9.5\end{array}$ & $\begin{array}{l}9.0 \\
9.0 \\
8.5 \\
9.0 \\
9.0\end{array}$ & $\begin{array}{l}9.5 \\
9.5 \\
9.0 \\
9.0 \\
9.5\end{array}$ & $\begin{array}{l}10.0 \\
11.0 \\
11.5 \\
11.5 \\
11.5\end{array}$ & $\begin{array}{r}9.5 \\
10.0 \\
11.0 \\
11.5 \\
10.5\end{array}$ & $\begin{array}{l}10.0 \\
10.5 \\
11.0 \\
11.5 \\
11.0\end{array}$ \\
\hline $\begin{array}{l}16 \\
17 \\
18 \\
19 \\
20\end{array}$ & $\begin{array}{l}19.5 \\
20.0 \\
20.0 \\
19.5 \\
19.0\end{array}$ & $\begin{array}{l}18.0 \\
18.5 \\
18.0 \\
18.0 \\
17.5\end{array}$ & $\begin{array}{l}19.0 \\
19.5 \\
19.5 \\
19.0 \\
18.5\end{array}$ & $\begin{array}{l}17.5 \\
17.0 \\
16.5 \\
17.0 \\
17.0\end{array}$ & $\begin{array}{l}17.0 \\
16.0 \\
15.5 \\
16.5 \\
16.5\end{array}$ & $\begin{array}{l}17.0 \\
16.5 \\
16.5 \\
16.5 \\
17.0\end{array}$ & $\begin{array}{l}9.5 \\
8.5 \\
8.5 \\
8.5 \\
9.0\end{array}$ & $\begin{array}{l}8.5 \\
7.5 \\
7.0 \\
7.5 \\
8.0\end{array}$ & $\begin{array}{l}9.0 \\
8.5 \\
8.5 \\
8.0 \\
8.5\end{array}$ & $\begin{array}{r}10.5 \\
10.0 \\
9.5 \\
9.5 \\
9.5\end{array}$ & $\begin{array}{r}10.0 \\
9.5 \\
9.0 \\
9.0 \\
9.0\end{array}$ & $\begin{array}{r}10.0 \\
9.5 \\
9.5 \\
9.0 \\
9.0\end{array}$ \\
\hline $\begin{array}{l}21 \\
22 \\
23 \\
24 \\
25\end{array}$ & $\begin{array}{l}18.5 \\
17.5 \\
18.5 \\
18.5 \\
16.5\end{array}$ & $\begin{array}{r}17.5 \\
12.0 \\
7.0 \\
.5 \\
.5\end{array}$ & $\begin{array}{r}18.0 \\
16.0 \\
15.0 \\
6.0 \\
12.0\end{array}$ & $\begin{array}{l}16.5 \\
16.0 \\
15.0 \\
14.5 \\
14.5\end{array}$ & $\begin{array}{l}16.0 \\
15.0 \\
14.5 \\
14.5 \\
14.0\end{array}$ & $\begin{array}{l}16.5 \\
15.5 \\
15.0 \\
14.5 \\
14.5\end{array}$ & $\begin{array}{r}9.0 \\
9.5 \\
10.5 \\
11.0 \\
10.5\end{array}$ & $\begin{array}{r}8.5 \\
9.0 \\
9.5 \\
10.0 \\
10.5\end{array}$ & $\begin{array}{r}9.0 \\
9.0 \\
9.5 \\
10.5 \\
10.5\end{array}$ & $\begin{array}{l}9.0 \\
9.0 \\
9.0 \\
9.0 \\
9.5\end{array}$ & $\begin{array}{l}9.0 \\
9.0 \\
8.5 \\
9.0 \\
9.0\end{array}$ & $\begin{array}{l}9.0 \\
9.0 \\
9.0 \\
9.0 \\
9.0\end{array}$ \\
\hline $\begin{array}{l}26 \\
27 \\
28 \\
29 \\
30 \\
31\end{array}$ & $\begin{array}{l}18.0 \\
18.5 \\
18.0 \\
17.5 \\
17.5 \\
16.0\end{array}$ & $\begin{array}{l}.5 \\
.5 \\
.5 \\
.5 \\
.5 \\
.5\end{array}$ & $\begin{array}{l}14.0 \\
16.5 \\
15.0 \\
13.0 \\
15.5 \\
12.5\end{array}$ & $\begin{array}{r}14.5 \\
14.5 \\
14.5 \\
13.5 \\
13.0 \\
-.-\end{array}$ & $\begin{array}{r}14.0 \\
14.5 \\
13.5 \\
13.0 \\
12.5 \\
-\end{array}$ & $\begin{array}{l}14.5 \\
14.5 \\
14.0 \\
13.0 \\
13.0 \\
\end{array}$ & $\begin{array}{l}11.0 \\
11.0 \\
11.5 \\
11.5 \\
11.5 \\
11.0\end{array}$ & $\begin{array}{l}10.5 \\
10.5 \\
11.0 \\
11.0 \\
11.0 \\
10.5\end{array}$ & $\begin{array}{l}10.5 \\
11.0 \\
11.5 \\
11.5 \\
11.0 \\
11.0\end{array}$ & $\begin{array}{r}9.5 \\
10.0 \\
10.5 \\
10.5 \\
11.5 \\
12.0\end{array}$ & $\begin{array}{r}9.5 \\
9.5 \\
10.0 \\
10.0 \\
10.5 \\
11.5\end{array}$ & $\begin{array}{r}9.5 \\
10.0 \\
10.0 \\
10.5 \\
11.0 \\
11.5\end{array}$ \\
\hline MONTH & 25.0 & .5 & 18.5 & 18.5 & .5 & 14.0 & 12.5 & 7.0 & 10.0 & 12.0 & 8.5 & 10.0 \\
\hline
\end{tabular}


SURFACE-WATER SITES--Cont 1 nued

02449000 TOMBIGBEE RIVER AT GAINESVILLE, AL--Cont1nued

TEMPERATURE, WATER (DEG. C), WATER YEAR OCTOBER 1988 TO SEPTEMBER 1989

\begin{tabular}{|c|c|c|c|c|c|c|c|c|c|c|c|c|}
\hline DAY & MAX & MIN & MEAN & MAX & MIN & MEAN & MAX & MIN & MEAN & MAX & MIN & MEAN \\
\hline \multicolumn{5}{|c|}{ FEBRUARY } & \multicolumn{2}{|c|}{ MARCH } & \multicolumn{3}{|c|}{ APRIL } & \multicolumn{3}{|c|}{ MAY } \\
\hline $\begin{array}{l}1 \\
2 \\
3 \\
4 \\
5\end{array}$ & $\begin{array}{l}12.5 \\
13.0 \\
13.5 \\
13.0 \\
12.5\end{array}$ & $\begin{array}{l}11.5 \\
12.5 \\
13.0 \\
12.5 \\
12.0\end{array}$ & $\begin{array}{l}12.0 \\
12.5 \\
13.0 \\
13.0 \\
12.5\end{array}$ & $\begin{array}{r}8.0 \\
8.5 \\
9.0 \\
9.5 \\
11.5\end{array}$ & $\begin{array}{l}7.5 \\
8.0 \\
8.5 \\
9.0 \\
9.5\end{array}$ & $\begin{array}{r}8.0 \\
8.0 \\
8.5 \\
9.0 \\
10.5\end{array}$ & $\begin{array}{l}17.0 \\
17.0 \\
17.5 \\
17.5 \\
17.5\end{array}$ & $\begin{array}{l}17.0 \\
17.0 \\
17.0 \\
17.0 \\
17.0\end{array}$ & $\begin{array}{l}17.0 \\
17.0 \\
17.0 \\
17.5 \\
17.5\end{array}$ & $\begin{array}{l}23.5 \\
23.5 \\
23.0 \\
23.0 \\
22.5\end{array}$ & $\begin{array}{l}22.5 \\
22.5 \\
23.0 \\
22.0 \\
22.0\end{array}$ & $\begin{array}{l}23.0 \\
23.0 \\
23.0 \\
22.5 \\
22.0\end{array}$ \\
\hline $\begin{array}{r}6 \\
7 \\
8 \\
9 \\
10\end{array}$ & $\begin{array}{r}12.0 \\
10.0 \\
7.5 \\
7.0 \\
6.0\end{array}$ & $\begin{array}{r}10.0 \\
7.5 \\
7.0 \\
6.0 \\
5.5\end{array}$ & $\begin{array}{r}11.5 \\
8.5 \\
7.0 \\
6.5 \\
6.0\end{array}$ & $\begin{array}{r}12.0 \\
11.0 \\
10.0 \\
9.0 \\
9.0\end{array}$ & $\begin{array}{r}11.0 \\
10.0 \\
9.0 \\
8.5 \\
8.5\end{array}$ & $\begin{array}{r}11.5 \\
10.5 \\
9.5 \\
8.5 \\
8.5\end{array}$ & $\begin{array}{l}17.0 \\
16.5 \\
16.0 \\
15.5 \\
14.5\end{array}$ & $\begin{array}{l}16.5 \\
16.0 \\
15.5 \\
14.5 \\
13.5\end{array}$ & $\begin{array}{l}16.5 \\
16.0 \\
15.5 \\
15.0 \\
14.0\end{array}$ & $\begin{array}{l}22.0 \\
20.5 \\
20.0 \\
20.0 \\
20.0\end{array}$ & $\begin{array}{l}21.0 \\
20.0 \\
19.5 \\
20.0 \\
19.5\end{array}$ & $\begin{array}{l}21.5 \\
20.5 \\
20.0 \\
20.0 \\
20.0\end{array}$ \\
\hline $\begin{array}{l}11 \\
12 \\
13 \\
14 \\
15\end{array}$ & $\begin{array}{r}6.0 \\
6.5 \\
7.5 \\
8.5 \\
10.0\end{array}$ & $\begin{array}{l}5.5 \\
6.0 \\
6.5 \\
7.5 \\
8.5\end{array}$ & $\begin{array}{l}6.0 \\
6.0 \\
7.0 \\
8.0 \\
9.0\end{array}$ & $\begin{array}{r}9.5 \\
11.0 \\
12.0 \\
13.0 \\
14.5\end{array}$ & $\begin{array}{r}9.0 \\
9.5 \\
11.0 \\
12.0 \\
13.5\end{array}$ & $\begin{array}{r}9.5 \\
10.0 \\
11.5 \\
12.5 \\
14.0\end{array}$ & $\begin{array}{l}14.0 \\
13.5 \\
14.0 \\
14.0 \\
14.5\end{array}$ & $\begin{array}{l}13.5 \\
13.0 \\
13.0 \\
14.0 \\
14.0\end{array}$ & $\begin{array}{l}13.5 \\
13.5 \\
13.5 \\
14.0 \\
14.5\end{array}$ & $\begin{array}{l}20.0 \\
20.0 \\
19.5 \\
19.5 \\
19.0\end{array}$ & $\begin{array}{l}19.5 \\
19.5 \\
19.5 \\
18.5 \\
18.5\end{array}$ & $\begin{array}{l}19.5 \\
19.5 \\
19.5 \\
19.0 \\
19.0\end{array}$ \\
\hline $\begin{array}{l}21 \\
22 \\
23 \\
24 \\
25\end{array}$ & $\begin{array}{r}11.5 \\
11.0 \\
10.5 \\
9.5 \\
8.0\end{array}$ & $\begin{array}{r}11.0 \\
10.5 \\
9.5 \\
8.0 \\
7.5\end{array}$ & $\begin{array}{r}11.0 \\
11.0 \\
10.0 \\
8.5 \\
7.5\end{array}$ & $\begin{array}{l}17.5 \\
16.5 \\
15.0 \\
14.0 \\
13.5\end{array}$ & $\begin{array}{l}16.5 \\
15.0 \\
14.0 \\
13.0 \\
13.0\end{array}$ & $\begin{array}{l}17.0 \\
16.0 \\
14.5 \\
13.5 \\
13.0\end{array}$ & $\begin{array}{l}18.5 \\
19.0 \\
19.5 \\
21.5 \\
21.5\end{array}$ & $\begin{array}{l}17.5 \\
18.0 \\
18.5 \\
19.0 \\
20.0\end{array}$ & $\begin{array}{l}18.0 \\
18.5 \\
19.0 \\
20.0 \\
20.5\end{array}$ & $\begin{array}{l}22.0 \\
22.5 \\
23.0 \\
23.5 \\
24.5\end{array}$ & $\begin{array}{l}21.5 \\
22.0 \\
22.5 \\
23.0 \\
23.5\end{array}$ & $\begin{array}{l}21.5 \\
22.5 \\
23.0 \\
23.5 \\
24.0\end{array}$ \\
\hline $\begin{array}{l}26 \\
27 \\
28 \\
29 \\
30 \\
31\end{array}$ & $\begin{array}{l}7.0 \\
7.0 \\
7.5 \\
-.- \\
--- \\
---\end{array}$ & \begin{tabular}{l}
7.0 \\
7.0 \\
7.0 \\
-- \\
\hdashline--
\end{tabular} & $\begin{array}{l}7.0 \\
7.0 \\
7.5 \\
--- \\
---\end{array}$ & $\begin{array}{l}13.5 \\
14.0 \\
15.0 \\
16.0 \\
17.0 \\
17.0\end{array}$ & $\begin{array}{l}13.0 \\
13.5 \\
14.0 \\
15.0 \\
16.0 \\
17.0\end{array}$ & $\begin{array}{l}13.5 \\
14.0 \\
14.5 \\
15.5 \\
16.5 \\
17.0\end{array}$ & $\begin{array}{r}22.0 \\
22.5 \\
22.5 \\
22.5 \\
23.0 \\
-\end{array}$ & $\begin{array}{r}20.5 \\
21.0 \\
21.5 \\
22.0 \\
22.0 \\
-\end{array}$ & $\begin{array}{r}21.0 \\
22.0 \\
22.0 \\
22.5 \\
22.5 \\
-\end{array}$ & $\begin{array}{l}25.0 \\
26.0 \\
26.5 \\
26.5 \\
27.0 \\
27.5\end{array}$ & $\begin{array}{l}24.0 \\
24.0 \\
25.0 \\
25.5 \\
25.5 \\
26.5\end{array}$ & $\begin{array}{l}24.5 \\
25.0 \\
25.5 \\
26.0 \\
26.5 \\
26.5\end{array}$ \\
\hline MONTH & 13.5 & 5.5 & 9.5 & 17.5 & 7.5 & 12.5 & 23.0 & 13.0 & 17.5 & 27.5 & 18.5 & 22.0 \\
\hline
\end{tabular}

JUNE JULY AUGUST

SEPTEMBER

\begin{tabular}{|c|c|c|c|c|c|c|}
\hline $\begin{array}{l}1 \\
2 \\
3 \\
4 \\
5\end{array}$ & $\begin{array}{l}28.0 \\
28.0 \\
28.0 \\
26.5 \\
27.0\end{array}$ & $\begin{array}{l}26.5 \\
27.0 \\
27.0 \\
26.0 \\
26.0\end{array}$ & $\begin{array}{l}27.0 \\
27.5 \\
27.5 \\
26.5 \\
26.5\end{array}$ & $\begin{array}{l}27.5 \\
27.0 \\
26.5 \\
26.0 \\
26.0\end{array}$ & $\begin{array}{l}26.0 \\
26.5 \\
26.0 \\
26.0 \\
26.0\end{array}$ & $\begin{array}{l}27.0 \\
27.0 \\
26.0 \\
26.0 \\
26.0\end{array}$ \\
\hline $\begin{array}{r}6 \\
7 \\
8 \\
9 \\
10\end{array}$ & $\begin{array}{l}26.5 \\
26.0 \\
26.0 \\
25.0 \\
24.0\end{array}$ & $\begin{array}{l}26.0 \\
25.5 \\
24.5 \\
23.5 \\
23.5\end{array}$ & $\begin{array}{l}26.5 \\
26.0 \\
25.5 \\
24.0 \\
23.5\end{array}$ & $\begin{array}{l}26.5 \\
27.0 \\
27.0 \\
27.5 \\
27.5\end{array}$ & $\begin{array}{l}26.0 \\
26.0 \\
26.5 \\
27.0 \\
27.0\end{array}$ & $\begin{array}{l}26.0 \\
26.5 \\
27.0 \\
27.0 \\
27.0\end{array}$ \\
\hline $\begin{array}{l}11 \\
12 \\
13 \\
14 \\
15\end{array}$ & $\begin{array}{l}24.0 \\
25.0 \\
25.5 \\
26.0 \\
25.5\end{array}$ & $\begin{array}{l}23.5 \\
24.0 \\
25.0 \\
25.5 \\
25.0\end{array}$ & $\begin{array}{l}24.0 \\
24.5 \\
25.0 \\
25.5 \\
25.5\end{array}$ & 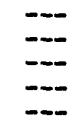 & $\begin{array}{l}-- \\
-- \\
-- \\
-- \\
--\end{array}$ & $\begin{array}{l}-- \\
-- \\
-- \\
--\end{array}$ \\
\hline $\begin{array}{l}16 \\
17 \\
18 \\
19 \\
20\end{array}$ & $\begin{array}{l}25.0 \\
24.0 \\
24.5 \\
24.5 \\
25.0\end{array}$ & $\begin{array}{l}24.0 \\
23.5 \\
24.0 \\
24.0 \\
24.5\end{array}$ & $\begin{array}{l}24.5 \\
24.0 \\
24.0 \\
24.5 \\
25.0\end{array}$ & 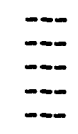 & $\begin{array}{l}-- \\
-- \\
-- \\
--\end{array}$ & $\begin{array}{l}--- \\
-- \\
-- \\
--\end{array}$ \\
\hline $\begin{array}{l}21 \\
22 \\
23 \\
24 \\
25\end{array}$ & $\begin{array}{l}25.5 \\
26.0 \\
26.0 \\
26.5 \\
26.5\end{array}$ & $\begin{array}{l}25.0 \\
25.5 \\
26.0 \\
26.0 \\
26.0\end{array}$ & $\begin{array}{l}25.5 \\
26.0 \\
26.0 \\
26.0 \\
26.5\end{array}$ & \begin{tabular}{l}
-- \\
\hdashline- \\
\hdashline- \\
-- \\
--
\end{tabular} & $\begin{array}{l}-- \\
-- \\
-\cdots \\
-\cdots\end{array}$ & $\begin{array}{l}--- \\
-- \\
-- \\
--\end{array}$ \\
\hline $\begin{array}{l}26 \\
27 \\
28 \\
29 \\
30 \\
31\end{array}$ & $\begin{array}{l}27.0 \\
27.0 \\
27.5 \\
27.5 \\
28.0\end{array}$ & $\begin{array}{r}26.5 \\
26.0 \\
26.5 \\
27.0 \\
27.0 \\
-\end{array}$ & $\begin{array}{r}26.5 \\
26.5 \\
27.0 \\
27.5 \\
27.5 \\
-\end{array}$ & $\begin{array}{l}\square- \\
\square- \\
\because- \\
-\square \\
-\square\end{array}$ & \begin{tabular}{l}
--- \\
$-\square$ \\
\hdashline-- \\
$-\square$ \\
$-\cdots$
\end{tabular} & $\begin{array}{l}-- \\
-- \\
-- \\
-- \\
-- \\
--\end{array}$ \\
\hline ONTH & 28.0 & 23.5 & 25.5 & - & --- & -- \\
\hline
\end{tabular}


SURFACE-WATER SITES--Cont 1 nued

323704087542400 TTW DEMOPOLIS LAKE ABOVE RATTLESNAKE BEND SR 12CA

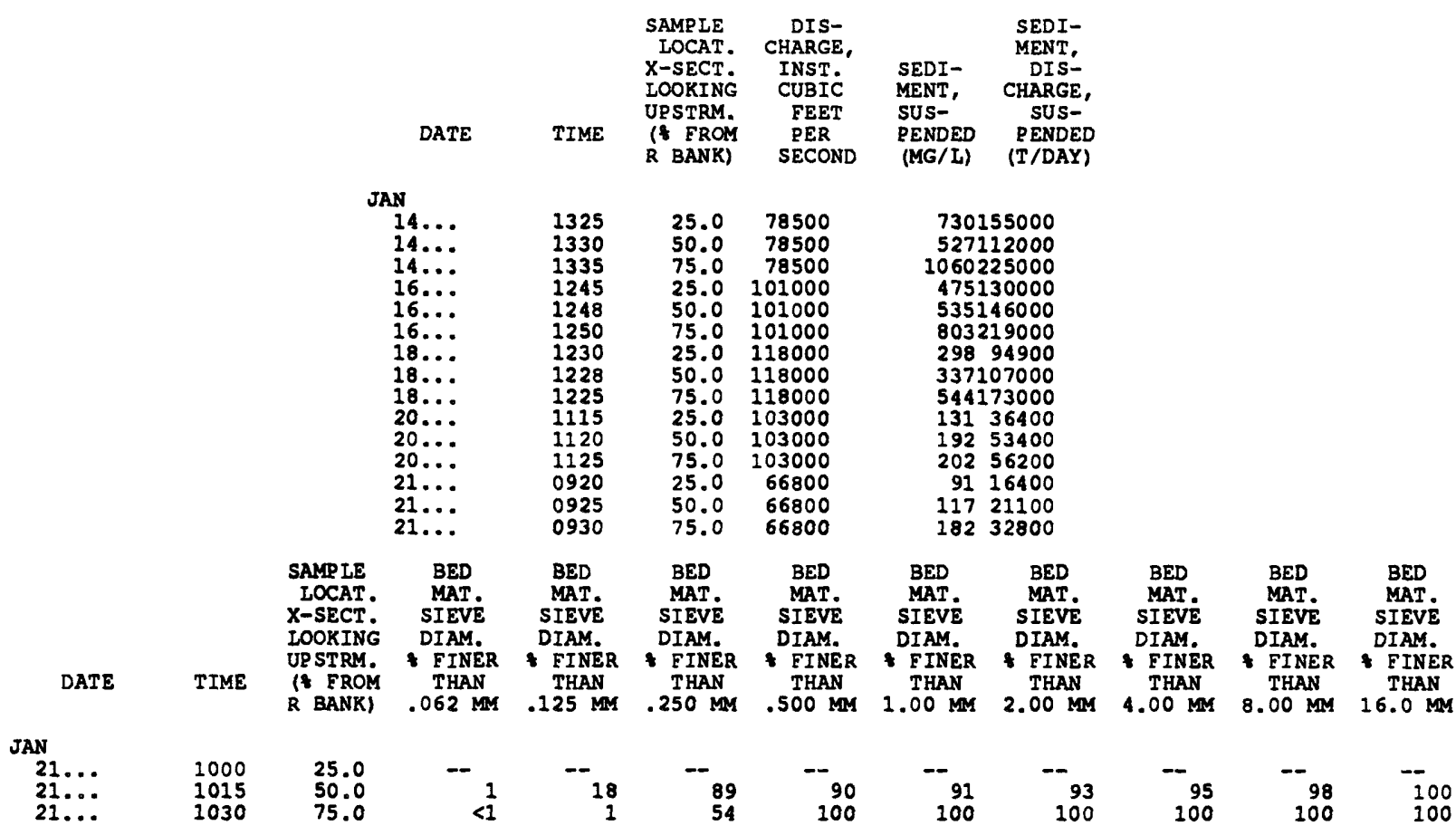

323653087540800 TTW DEMOPOLIS LAKE RATTIESNAKE BEND SR RBI

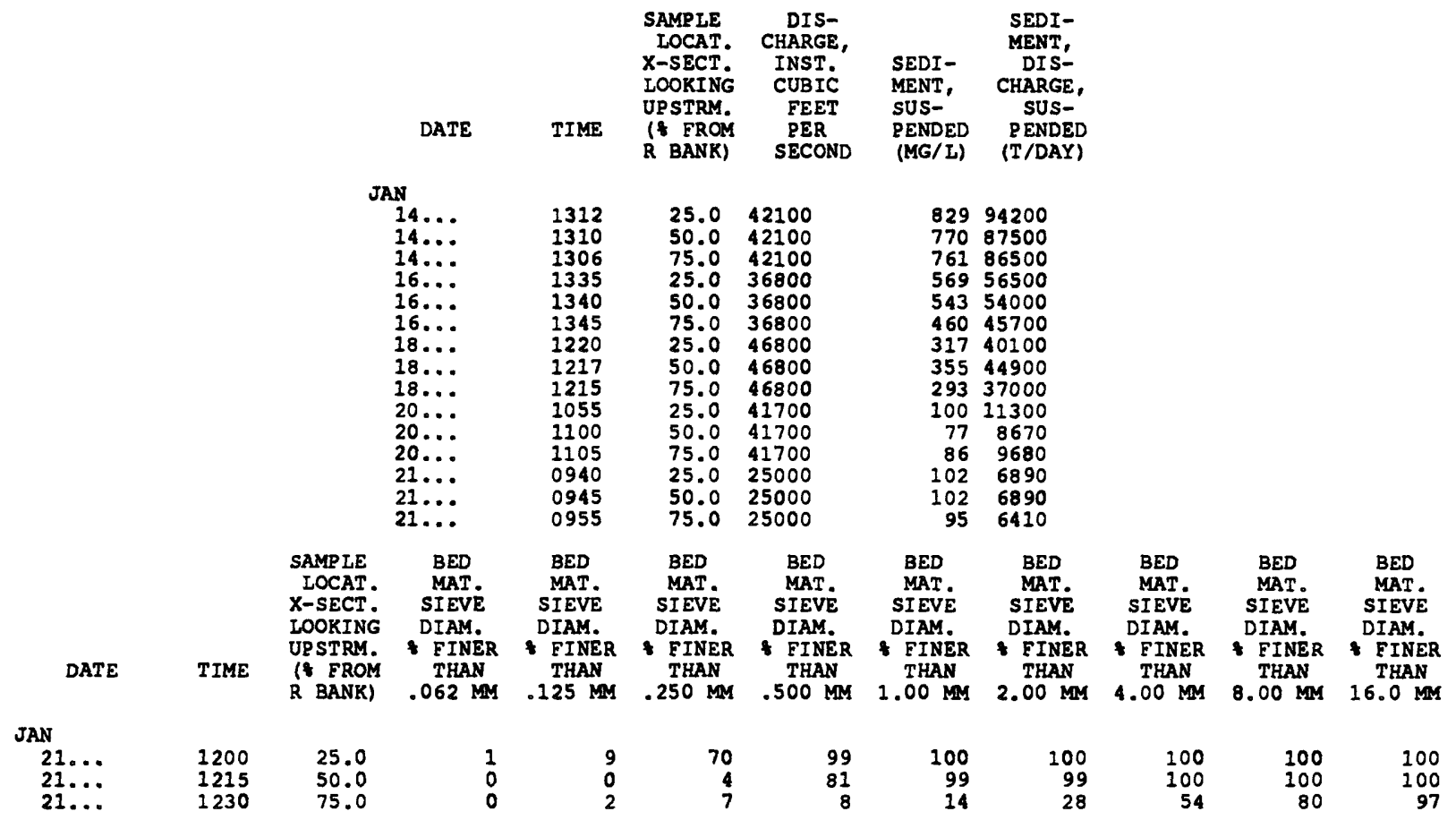


SUREACE-WATER SITES--Cont1 nued

323642087541800 TTW DEMOPOLIS LAKE RATTLESNAKE CUT SR 12CB

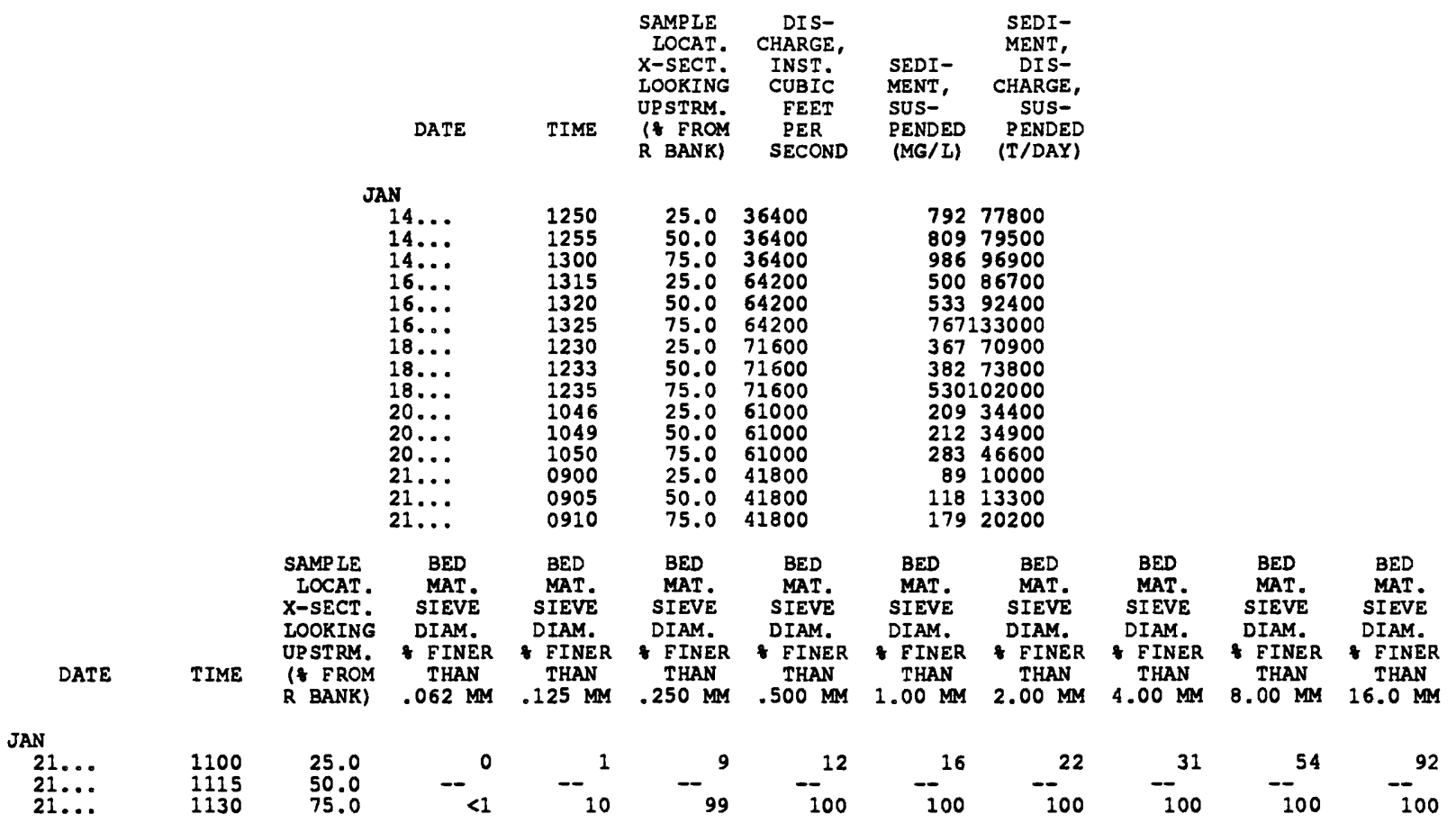

02467001 TOMBIGBEE RIVER BELOW DEMOPOLIS LOCK AND DAM, AI

\begin{tabular}{|c|c|c|c|c|c|c|c|}
\hline DATE & TIME & $\begin{array}{l}\text { SAM- } \\
\text { PLING } \\
\text { DEPTH } \\
\text { (FEET) }\end{array}$ & $\begin{array}{l}\text { SPE- } \\
\text { CIEIC } \\
\text { CON- } \\
\text { DUCT- } \\
\text { ANCE } \\
\text { (US/CM) }\end{array}$ & $\begin{array}{c}\text { PH } \\
\text { (STAND- } \\
\text { ARD } \\
\text { UNITS) }\end{array}$ & $\begin{array}{c}\text { TEMPER- } \\
\text { ATURE } \\
\text { WATER } \\
\text { (DEG C) }\end{array}$ & $\begin{array}{c}\text { OXYGEN, } \\
\text { DIS- } \\
\text { SOLVED } \\
\text { (MG/I) }\end{array}$ & $\begin{array}{c}\text { OXYGEN, } \\
\text { DIS- } \\
\text { SOLVED } \\
\text { (PER- } \\
\text { CENT } \\
\text { SATUR- } \\
\text { ATION) }\end{array}$ \\
\hline \multicolumn{8}{|l|}{ DEC } \\
\hline $\begin{array}{l}07 \ldots \\
07 \ldots \\
07 \ldots \\
07 \ldots \\
07 \ldots \\
07 \ldots \\
\text { MAR }\end{array}$ & $\begin{array}{l}1215 \\
1216 \\
1217 \\
1218 \\
1219 \\
1220\end{array}$ & $\begin{array}{l}-- \\
5.00 \\
10.0 \\
15.0 \\
20.0 \\
25.0\end{array}$ & $\begin{array}{l}190 \\
190 \\
200 \\
200 \\
200 \\
200\end{array}$ & $\begin{array}{l}6.9 \\
7.1 \\
7.1 \\
7.2 \\
7.2 \\
7.2\end{array}$ & $\begin{array}{l}12.5 \\
12.5 \\
13.0 \\
12.5 \\
12.5 \\
12.5\end{array}$ & $\begin{array}{l}12.4 \\
11.2 \\
11.0 \\
11.0 \\
11.2 \\
11.1\end{array}$ & $\begin{array}{l}116 \\
-- \\
-- \\
-- \\
--\end{array}$ \\
\hline $\begin{array}{l}02 \ldots \\
02 \ldots \\
02 \ldots \\
02 \ldots \\
02 \ldots \\
02 \ldots \\
02 \ldots \\
02 \ldots \\
02 \ldots \\
02 \ldots \\
02 \ldots \\
02 \ldots \\
02 \ldots \\
\text { JuN }\end{array}$ & $\begin{array}{l}1400 \\
1401 \\
1402 \\
1403 \\
1404 \\
1405 \\
1406 \\
1407 \\
1408 \\
1409 \\
1410 \\
1411 \\
1412\end{array}$ & $\begin{array}{l}-- \\
5.00 \\
10.0 \\
15.0 \\
20.0 \\
25.0 \\
30.0 \\
35.0 \\
40.0 \\
45.0 \\
50.0 \\
55.0 \\
60.0\end{array}$ & $\begin{array}{l}124 \\
123 \\
122 \\
122 \\
122 \\
122 \\
123 \\
123 \\
122 \\
121 \\
121 \\
120 \\
120\end{array}$ & $\begin{array}{l}7.7 \\
7.5 \\
7.5 \\
7.5 \\
7.6 \\
7.5 \\
7.6 \\
7.5 \\
7.5 \\
7.5 \\
7.5 \\
7.5 \\
7.5\end{array}$ & $\begin{array}{l}9.0 \\
8.5 \\
8.5 \\
8.5 \\
8.5 \\
8.5 \\
8.5 \\
8.5 \\
8.5 \\
8.5 \\
8.5 \\
8.5 \\
8.5\end{array}$ & $\begin{array}{l}13.5 \\
13.4 \\
13.0 \\
12.9 \\
12.9 \\
12.9 \\
12.8 \\
12.9 \\
12.8 \\
12.8 \\
12.5 \\
12.7 \\
12.7\end{array}$ & $\begin{array}{l}117 \\
=- \\
-- \\
-- \\
-- \\
=- \\
-- \\
-- \\
-- \\
=- \\
=\end{array}$ \\
\hline $\begin{array}{c}08 \ldots \\
08 \ldots \\
08 \ldots \\
08 \ldots \\
08 \ldots \\
08 \ldots \\
08 \ldots \\
08 \ldots \\
\text { AUG }\end{array}$ & $\begin{array}{l}0745 \\
0746 \\
0747 \\
0748 \\
0749 \\
0750 \\
0751 \\
0752\end{array}$ & $\begin{array}{l}-- \\
5.00 \\
10.0 \\
15.0 \\
20.0 \\
25.0 \\
30.0 \\
33.0\end{array}$ & $\begin{array}{l}144 \\
143 \\
143 \\
142 \\
141 \\
140 \\
139 \\
138\end{array}$ & $\begin{array}{l}7.3 \\
7.3 \\
7.3 \\
7.2 \\
7.3 \\
7.3 \\
7.3 \\
7.3\end{array}$ & $\begin{array}{l}27.0 \\
27.0 \\
27.0 \\
27.0 \\
27.0 \\
27.0 \\
27.0 \\
27.0\end{array}$ & $\begin{array}{l}8.0 \\
7.6 \\
7.6 \\
7.9 \\
7.6 \\
7.6 \\
7.7 \\
7.6\end{array}$ & $\begin{array}{l}102 \\
-- \\
-- \\
-- \\
-- \\
-- \\
--\end{array}$ \\
\hline $\begin{array}{l}10 \ldots \\
10 \ldots \\
10 \ldots \\
10 \ldots \\
10 \ldots\end{array}$ & $\begin{array}{l}0830 \\
0831 \\
0832 \\
0833 \\
0834\end{array}$ & $\begin{array}{c}-- \\
5.00 \\
10.0 \\
15.0 \\
20.0\end{array}$ & $\begin{array}{l}136 \\
136 \\
136 \\
135 \\
135\end{array}$ & $\begin{array}{l}7.4 \\
7.4 \\
7.4 \\
7.4 \\
7.4\end{array}$ & $\begin{array}{l}29.5 \\
29.5 \\
29.5 \\
29.5 \\
29.5\end{array}$ & $\begin{array}{l}7.6 \\
7.1 \\
7.0 \\
6.7 \\
6.6\end{array}$ & $\begin{array}{l}100 \\
=- \\
=- \\
=-\end{array}$ \\
\hline
\end{tabular}


SURFACE-WATER SITES--COnt1 nued

02467001 TOMBIGBEE RIVER BELOW DEMOPOLIS LOCK AND DAM, AL--Cont Inued

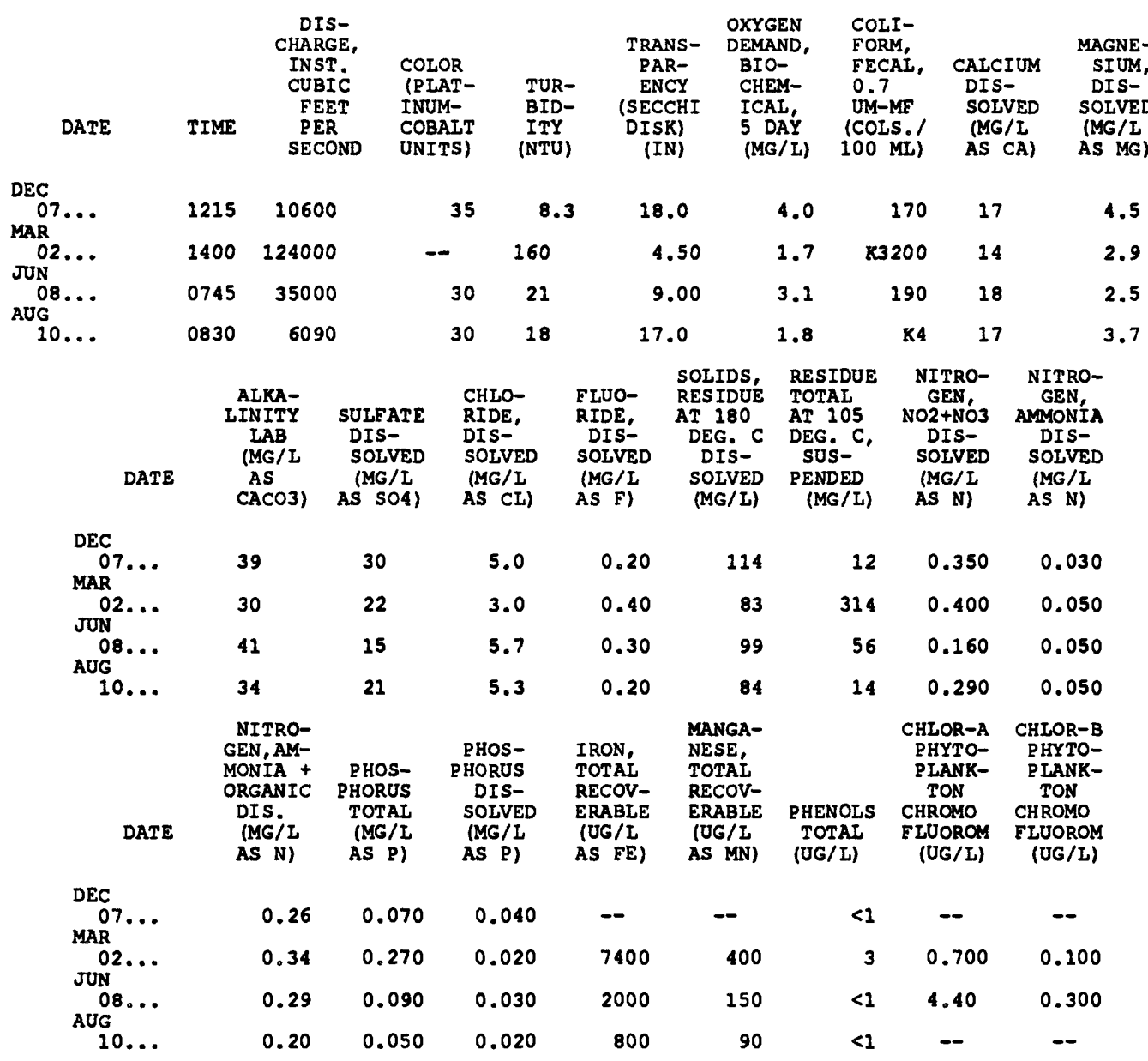

02469762 TOMBIGBEE RIVER BELON COFEEEVILLE LOCK AND DAM, AL

\begin{tabular}{|c|c|c|c|c|c|c|c|c|c|c|c|c|}
\hline DATE & TIME & $\begin{array}{l}\text { DIS- } \\
\text { CHARGE, } \\
\text { INST. } \\
\text { CUBIC } \\
\text { FEET } \\
\text { PER } \\
\text { SECOND }\end{array}$ & $\begin{array}{l}\text { SPE- } \\
\text { CIFIC } \\
\text { CON- } \\
\text { DUCT- } \\
\text { ANCE } \\
\text { (US/CM) }\end{array}$ & $\begin{array}{c}\text { PH } \\
\text { (STAND- } \\
\text { ARD } \\
\text { UNITS) }\end{array}$ & $\begin{array}{c}\text { TEMPER- } \\
\text { ATURE } \\
\text { WATER } \\
\text { (DEG C) }\end{array}$ & $\begin{array}{l}\text { TUR- } \\
\text { BID- } \\
\text { ITY } \\
\text { (NTU) }\end{array}$ & $\begin{array}{c}\text { OXYGEN, } \\
\text { DIS- } \\
\text { SOIVED } \\
\text { (MG/L) }\end{array}$ & $\begin{array}{c}\text { OXYGEN, } \\
\text { DIS- } \\
\text { SOLVED } \\
\text { (PER- } \\
\text { CENT } \\
\text { SATUR- } \\
\text { ATION) }\end{array}$ & $\begin{array}{l}\text { COLI- } \\
\text { FORM, } \\
\text { FECAL, } \\
0.7 \\
\text { UM-MF } \\
\text { (COLS.' } \\
100 \mathrm{ML} \text { ) }\end{array}$ & $\begin{array}{c}\text { STREP- } \\
\text { TOCOCCI } \\
\text { FECAL, } \\
\text { KF AGAR } \\
\text { (COLS. } \\
\text { PER } \\
100 \mathrm{ML} \text { ) }\end{array}$ & $\begin{array}{l}\text { HARD- } \\
\text { NESS } \\
\text { TOTAL } \\
\text { (MG/L } \\
\text { AS } \\
\text { CACO3) }\end{array}$ & $\begin{array}{l}\text { CALCIUM } \\
\text { DIS- } \\
\text { SOLVED } \\
\text { (MG/L } \\
\text { AS CA) }\end{array}$ \\
\hline
\end{tabular}

\begin{tabular}{|c|c|c|c|c|c|c|c|c|c|c|c|c|}
\hline \multirow{4}{*}{$\begin{array}{l}\text { OCT } \\
20 \ldots \\
\text { EEB } \\
23 \ldots \\
\text { APR } \\
13 \ldots \\
\text { AUG } \\
17 \ldots\end{array}$} & 1025 & \multicolumn{2}{|c|}{4910} & 238 & 7.6 & 21.0 & 11 & 9.3 & 104 & 5 & 13 & 72 \\
\hline & 1040 & 67200 & & 128 & 7.3 & 9.0 & 77 & 11.8 & 101 & - & 710 & 57 \\
\hline & 1020 & 62800 & & 132 & 7.3 & 15.0 & 39 & 9.0 & 89 & $\mathrm{~K} 160$ & 270 & 47 \\
\hline & 1000 & 4620 & & 146 & 7.4 & 29.0 & 16 & 8.0 & 104 & 26 & 32 & 50 \\
\hline DATE & & $\begin{array}{l}\text { NE- } \\
\text { UM, } \\
\text { S- } \\
\text { VED } \\
\text { II } \\
\text { MG) }\end{array}$ & $\begin{array}{l}\text { SODIUM, } \\
\text { DIS- } \\
\text { SOLVED } \\
\text { (MG/L } \\
\text { AS NA) }\end{array}$ & $\begin{array}{r}\text { SODIUM } \\
\text { PERCENT }\end{array}$ & $\begin{array}{c}\text { SODIUM } \\
\text { AD- } \\
\text { SORP- } \\
\text { TION } \\
\text { RATIO }\end{array}$ & $\begin{array}{c}\text { POTAS- } \\
\text { SIUM, } \\
\text { DIS- } \\
\text { SOIVED } \\
\text { (MG/I } \\
\text { AS K) }\end{array}$ & $\begin{array}{c}\text { BICAR- } \\
\text { BONATE } \\
\text { WATER } \\
\text { DIS IT } \\
\text { FIELD } \\
\text { MG/L AS } \\
\text { HCO3 }\end{array}$ & $\begin{array}{l}\text { ALKA- } \\
\text { IINITY } \\
\text { WAT DIS } \\
\text { TOT IT } \\
\text { FIELD } \\
\text { MG/L AS } \\
\text { CACO3 }\end{array}$ & $\begin{array}{l}\text { SULFATE } \\
\text { DIS- } \\
\text { SOLVED } \\
\text { (MG/L } \\
\text { AS SO4) }\end{array}$ & $\begin{array}{l}\text { CHLO- } \\
\text { RIDE, } \\
\text { DIS- } \\
\text { SOLVED } \\
\text { (MG/L } \\
\text { AS CL) }\end{array}$ & $\begin{array}{l}\text { FLUO- } \\
\text { RIDE, } \\
\text { DIS- } \\
\text { SOLVED } \\
\text { (MG/L } \\
\text { AS F) }\end{array}$ & $\begin{array}{c}\text { SILICA, } \\
\text { DIS- } \\
\text { SOLVED } \\
\text { (MG/I } \\
\text { AS } \\
\text { SIO2) }\end{array}$ \\
\hline
\end{tabular}

\begin{tabular}{|c|c|c|c|c|c|c|c|c|c|c|c|}
\hline $\begin{array}{l}\text { OCT } \\
20 \ldots \\
\text { FEB }\end{array}$ & 4.8 & 18 & 34 & 0.9 & 2.7 & 61 & 50 & 34 & 17 & 0.10 & 5.7 \\
\hline${ }_{A P R}^{23 \ldots}$ & 2.9 & 5.4 & 17 & 0.3 & 1.6 & 43 & 35 & 29 & 7.9 & 0.10 & 7.2 \\
\hline AUG $13 \ldots$ & 3.0 & 5.0 & 18 & 0.3 & 1.6 & 38 & 31 & 16 & 4.3 & 0.10 & 8.0 \\
\hline $17 \ldots$ & 2.9 & 8.6 & 27 & 0.5 & 1.8 & 44 & 36 & 17 & 9.1 & 0.10 & 7.1 \\
\hline
\end{tabular}


SURFACE-WATER SITES--Continued

02469762 TOMBIGBEE RIVER BELOW COFFEEVILLE LOCK AND DAM, AL--Cont1nued

\begin{tabular}{|c|c|c|c|c|c|c|c|c|c|c|c|}
\hline DATE & $\begin{array}{l}\text { SOLIDS, } \\
\text { RESIDUE } \\
\text { AT } 180 \\
\text { DEG. C } \\
\text { DIS- } \\
\text { SOLVED } \\
\text { (MG/L) }\end{array}$ & $\begin{array}{l}\text { SOIIDS, } \\
\text { SUM OF } \\
\text { CONSTI- } \\
\text { TUENTS, } \\
\text { DIS- } \\
\text { SOLVED } \\
\text { (MG/L) }\end{array}$ & $\begin{array}{c}\text { SOLIDS, } \\
\text { DIS- } \\
\text { SOLVED } \\
\text { (TONS } \\
\text { PER } \\
\text { AC-FT) }\end{array}$ & $\begin{array}{l}\text { SOIIDS, } \\
\text { DIS- } \\
\text { SOIVED } \\
\text { (TONS } \\
\text { PER } \\
\text { DAY) }\end{array}$ & $\begin{array}{c}\text { NITRO- } \\
\text { GEN, } \\
\text { NO2+NO3 } \\
\text { DIS- } \\
\text { SOIVED } \\
\text { (MG/I } \\
\text { AS N) }\end{array}$ & $\begin{array}{l}\text { NITRO- } \\
\text { GEN, } \\
\text { AMMONIA } \\
\text { DIS- } \\
\text { SOLVED } \\
\text { (MG/L } \\
\text { AS N) }\end{array}$ & $\begin{array}{l}\text { NITRO- } \\
\text { GEN, AM- } \\
\text { MONIA + } \\
\text { ORGANIC } \\
\text { TOTAL } \\
\text { (MG/L } \\
\text { AS N) }\end{array}$ & $\begin{array}{l}\text { PHOS- } \\
\text { PHORUS } \\
\text { TOTAL } \\
\text { (MG/I } \\
\text { AS P) }\end{array}$ & $\begin{array}{l}\text { PHOS- } \\
\text { PHORUS } \\
\text { DIS- } \\
\text { SOIVED } \\
\text { (MG/I } \\
\text { AS P) }\end{array}$ & $\begin{array}{l}\text { PHOS- } \\
\text { PHORUS } \\
\text { ORTHO, } \\
\text { DIS- } \\
\text { SOLVED } \\
\text { (MG/I } \\
\text { AS P) }\end{array}$ & $\begin{array}{l}\text { ALUM- } \\
\text { INUM, } \\
\text { DIS- } \\
\text { SOLVED } \\
\text { (UG/I } \\
\text { AS AL) }\end{array}$ \\
\hline ... & 141 & 135 & 0.19 & 1870 & 0.300 & 0.010 & 0.70 & 0.040 & 0.030 & 0.020 & 10 \\
\hline$\cdots$ & 163 & 97 & 0.22 & 29600 & 0.290 & 0.090 & 0.30 & 0.200 & 0.050 & 0.070 & 930 \\
\hline . & 84 & 72 & 0.11 & 14200 & 0.220 & 0.050 & 0.50 & 0.070 & 0.030 & $<0.010$ & 70 \\
\hline$\cdots$ & 100 & 85 & 0.14 & 1250 & 0.250 & 0.030 & 0.40 & 0.060 & 0.020 & 0.010 & 40 \\
\hline DATE & $\begin{array}{c}\text { ARSENIC } \\
\text { DIS- } \\
\text { SOLVED } \\
\text { (UG/L } \\
\text { AS AS) }\end{array}$ & $\begin{array}{l}\text { BARIUM, } \\
\text { DIS- } \\
\text { SOIVED } \\
\text { (UG/L } \\
\text { AS BA) }\end{array}$ & $\begin{array}{l}\text { BERYL- } \\
\text { LIUM, } \\
\text { DIS- } \\
\text { SOLVED } \\
\text { (UG/L } \\
\text { AS BE) }\end{array}$ & $\begin{array}{c}\text { CADMIUM } \\
\text { DIS- } \\
\text { SOLVED } \\
\text { (UG/I } \\
\text { AS CD) }\end{array}$ & $\begin{array}{l}\text { CHRO- } \\
\text { MIUM, } \\
\text { DIS- } \\
\text { SOIVED } \\
\text { (UG/I } \\
\text { AS CR) }\end{array}$ & $\begin{array}{l}\text { COBALT, } \\
\text { DIS- } \\
\text { SOLVED } \\
\text { (UG/L } \\
\text { AS CO) }\end{array}$ & $\begin{array}{l}\text { CORPER, } \\
\text { DIS- } \\
\text { SOLVED } \\
\text { (UG/I } \\
\text { AS CU) }\end{array}$ & $\begin{array}{l}\text { IRON, } \\
\text { DIS- } \\
\text { SOIVED } \\
\text { (UG/I } \\
\text { AS FE) }\end{array}$ & $\begin{array}{l}\text { IEAD, } \\
\text { DIS- } \\
\text { SOIVED } \\
\text { (UG/I } \\
\text { AS PB) }\end{array}$ & $\begin{array}{c}\text { LITHIUM } \\
\text { DIS- } \\
\text { SOLVED } \\
\text { (UG/I } \\
\text { AS II) }\end{array}$ & $\begin{array}{l}\text { MANGA- } \\
\text { NESE, } \\
\text { DIS- } \\
\text { SOLVED } \\
\text { (UG/L } \\
\text { AS MN) }\end{array}$ \\
\hline ... & 1 & 39 & $<0.5$ & 1.0 & $<1$ & $<3$ & 2 & 180 & $<5$ & 8 & 11 \\
\hline 3... & 1 & 43 & $<0.5$ & $<1.0$ & $<1$ & $<3$ & 2 & 1300 & $<5$ & $<4$ & 160 \\
\hline $3 \ldots$ & $<1$ & 30 & $<0.5$ & 3.0 & $<1$ & $<3$ & 7 & 350 & $<5$ & $<4$ & 12 \\
\hline $7 \ldots$ & 1 & 27 & $<0.5$ & $<1.0$ & $<1$ & $<3$ & $<1$ & 150 & $\cdot<1$ & $<4$ & 15 \\
\hline DATE & $\begin{array}{c}\text { MERCURY } \\
\text { DIS- } \\
\text { SOLVED } \\
\text { (UG/L } \\
\text { AS HG) }\end{array}$ & $\begin{array}{l}\text { MOLYB- } \\
\text { DENUM, } \\
\text { DIS- } \\
\text { SOLVED } \\
\text { (UG/L } \\
\text { AS MO) }\end{array}$ & $\begin{array}{l}\text { NICKEL, } \\
\text { DIS- } \\
\text { SOLVED } \\
\text { (UG/I } \\
\text { AS NI) }\end{array}$ & $\begin{array}{l}\text { SELE- } \\
\text { NIUM, } \\
\text { DIS- } \\
\text { SOLVED } \\
\text { (UG/I } \\
\text { AS SE) }\end{array}$ & $\begin{array}{c}\text { SILVER, } \\
\text { DIS- } \\
\text { SOLVED } \\
\text { (UG/I } \\
\text { AS AG) }\end{array}$ & $\begin{array}{l}\text { STRON- } \\
\text { TIUM, } \\
\text { DIS- } \\
\text { SOLVED } \\
\text { (UG/L } \\
\text { AS SR) }\end{array}$ & $\begin{array}{l}\text { VANA- } \\
\text { DIUM, } \\
\text { DIS- } \\
\text { SOLVED } \\
\text { (UG/I } \\
\text { AS V) }\end{array}$ & $\begin{array}{l}\text { ZINC, } \\
\text { DIS- } \\
\text { SOLVED } \\
\text { (UG/L } \\
\text { AS } 2 N \text { ) }\end{array}$ & $\begin{array}{l}\text { SEDI- } \\
\text { MENT, } \\
\text { SUS- } \\
\text { PENDED } \\
\text { (MG/I) }\end{array}$ & $\begin{array}{l}\text { SEDI- } \\
\text { MENT, } \\
\text { DIS- } \\
\text { CHARGE, } \\
\text { SUS- } \\
\text { PENDED } \\
\text { (T/DAY) }\end{array}$ & $\begin{array}{c}\text { SED. } \\
\text { SUSP. } \\
\text { SIEVE } \\
\text { DIAM. } \\
\text { FINER } \\
\text { FIHAN } \\
.062 \mathrm{MM}\end{array}$ \\
\hline
\end{tabular}

\begin{tabular}{|c|c|c|c|c|c|c|c|c|c|c|}
\hline $\begin{array}{l}\mathrm{OCT} \\
20 \ldots \\
\text { FEB }\end{array}$ & $<0.1$ & $<10$ & $<1$ & $<1$ & $<1.0$ & 120 & $<6$ & 14 & 90 & 99 \\
\hline${ }_{A P R}^{23} \cdots$ & $<0.1$ & $<10$ & $<1$ & $<1$ & $<1.0$ & 110 & $<6$ & 41 & 22941500 & 76 \\
\hline AUG $13 \ldots$ & 0.1 & $<10$ & $<1$ & $<1$ & $<1.0$ & 89 & $<6$ & 8 & $178 \quad 30200$ & 65 \\
\hline $17 \ldots$ & $<0.1$ & $<10$ & $<1$ & $<1$ & $<1.0$ & 92 & $<6$ & 7 & 237 & 99 \\
\hline$\pi^{-\infty}$ & DATE & $\begin{array}{l}\text { GROSS } \\
\text { ALPHA, } \\
\text { DIS- } \\
\text { SOLVED } \\
\text { (UG/L } \\
\text { AS } \\
\text { U-NAT) }\end{array}$ & $\begin{array}{l}\text { GROSS } \\
\text { ALPHA, } \\
\text { SUSP } \\
\text { TOTAL } \\
\text { (UG/L } \\
\text { AS } \\
\text { U-NAT) }\end{array}$ & $\begin{array}{l}\text { GROSS } \\
\text { BETA, } \\
\text { DIS- } \\
\text { SOIVED } \\
\text { (PCI/L } \\
\text { AS } \\
\text { CS-137) }\end{array}$ & $\begin{array}{c}\text { GROSS } \\
\text { BETA, } \\
\text { SUSP } \\
\text { TOTAL } \\
\text { (PCI } / 1 \\
\text { AS } \\
\text { CS-137) }\end{array}$ & $\begin{array}{l}\text { GROSS } \\
\text { BETA, } \\
\text { DIS- } \\
\text { SOLVED } \\
\text { (PCI/L } \\
\text { AS SR/ } \\
\text { YT-90) }\end{array}$ & $\begin{array}{l}\text { GROSS } \\
\text { BETA, } \\
\text { SUSP. } \\
\text { TOTAL } \\
\text { (PCI/L } \\
\text { AS SR/ } \\
\text { YT-90) }\end{array}$ & $\begin{array}{l}\text { RADIUM } \\
226, \\
\text { DIS- } \\
\text { SOIVED, } \\
\text { RADON } \\
\text { METHOD } \\
\text { (PCI/L) }\end{array}$ & $\begin{array}{l}\text { URANIUM } \\
\text { NATURAL } \\
\text { DIS- } \\
\text { SOLVED } \\
\text { (UG/L } \\
\text { AS U) }\end{array}$ & \\
\hline
\end{tabular}

\begin{tabular}{|c|c|c|c|c|c|c|c|c|}
\hline $\begin{array}{l}\mathrm{FEB} \\
23 \ldots\end{array}$ & 4.0 & 10 & 2.0 & 6.2 & 1.7 & 5.6 & 0.04 & 0.14 \\
\hline $17 \ldots$ & $<0.4$ & 1.5 & 4.0 & 1.2 & 3.2 & 1.0 & 0.10 & 0.36 \\
\hline
\end{tabular}


02469762 TOMBIGBEE RIVER BELOW COFFEEVILLE LOCK AND DAM, AL--Cont Inued

SPECIFIC CONDUCTANCE, MICROSIEMENS PER CENTIMETER AT 25 DEG. C, WATER YEAR OCTOBER 1988 TO SEPTEMBER 1989

\begin{tabular}{|c|c|c|c|c|c|c|c|c|c|c|c|c|}
\hline DAY & OCT & NOV & DEC & JAN & FEB & MAR & APR & MAY & JUN & JUL & AUG & SEP \\
\hline $\begin{array}{l}1 \\
2 \\
3 \\
4 \\
5\end{array}$ & $\begin{array}{l}210 \\
222 \\
198 \\
192 \\
190\end{array}$ & $\begin{array}{l}210 \\
205 \\
164 \\
165 \\
151\end{array}$ & $\begin{array}{l}167 \\
164 \\
160 \\
152 \\
155\end{array}$ & $\begin{array}{l}121 \\
135 \\
138 \\
129 \\
139\end{array}$ & $\begin{array}{l}-117 \\
110 \\
122 \\
123\end{array}$ & $\begin{array}{r}-117 \\
122 \\
115 \\
97\end{array}$ & $\begin{array}{r}97 \\
108 \\
112 \\
115 \\
111\end{array}$ & $\begin{array}{l}143 \\
133 \\
138 \\
141 \\
149\end{array}$ & $\begin{array}{l}145 \\
145 \\
146 \\
151 \\
146\end{array}$ & $\begin{array}{r}--- \\
--- \\
92 \\
122\end{array}$ & $\begin{array}{l}102 \\
107 \\
106 \\
104 \\
111\end{array}$ & $\begin{array}{l}115 \\
124 \\
123 \\
118 \\
123\end{array}$ \\
\hline $\begin{array}{r}6 \\
7 \\
8 \\
9 \\
10\end{array}$ & $\begin{array}{l}187 \\
173 \\
168 \\
162 \\
158\end{array}$ & $\begin{array}{l}155 \\
161 \\
192 \\
174 \\
176\end{array}$ & $\begin{array}{l}153 \\
156 \\
152 \\
150 \\
154\end{array}$ & $\begin{array}{l}131 \\
127 \\
124 \\
123 \\
-\end{array}$ & $\begin{array}{l}103 \\
124 \\
129 \\
138 \\
141\end{array}$ & $\begin{array}{r}102 \\
117 \\
107 \\
104 \\
95\end{array}$ & $\begin{array}{r}110 \\
118 \\
121 \\
73 \\
118\end{array}$ & $\begin{array}{l}150 \\
-137 \\
--- \\
--\end{array}$ & $\begin{array}{l}161 \\
160 \\
144 \\
135 \\
131\end{array}$ & $\begin{array}{r}126 \\
122 \\
125 \\
109 \\
86\end{array}$ & $\begin{array}{r}105 \\
103 \\
96 \\
110 \\
97\end{array}$ & $\begin{array}{l}124 \\
127 \\
129 \\
136 \\
133\end{array}$ \\
\hline $\begin{array}{l}11 \\
12 \\
13 \\
14 \\
15\end{array}$ & $\begin{array}{l}162 \\
160 \\
178 \\
176 \\
181\end{array}$ & $\begin{array}{l}178 \\
176 \\
197 \\
176 \\
182\end{array}$ & $\begin{array}{l}158 \\
165 \\
164 \\
168 \\
-\end{array}$ & $\begin{array}{l}146 \\
131 \\
--- \\
--- \\
--\end{array}$ & $\begin{array}{l}142 \\
140 \\
141 \\
150 \\
161\end{array}$ & $\begin{array}{r}92 \\
91 \\
115 \\
101 \\
107\end{array}$ & $\begin{array}{r}79 \\
122 \\
134 \\
154 \\
164\end{array}$ & $\begin{array}{l}142 \\
149 \\
142 \\
133 \\
138\end{array}$ & $\begin{array}{l}138 \\
148 \\
146 \\
149\end{array}$ & $\begin{array}{l}82 \\
80 \\
80 \\
78 \\
78\end{array}$ & $\begin{array}{r}85 \\
104 \\
105 \\
101 \\
109\end{array}$ & $\begin{array}{l}128 \\
128 \\
139 \\
135 \\
134\end{array}$ \\
\hline $\begin{array}{l}16 \\
17 \\
18 \\
19 \\
20\end{array}$ & $\begin{array}{l}180 \\
179 \\
188 \\
192 \\
190\end{array}$ & $\begin{array}{l}180 \\
180 \\
170 \\
175 \\
158\end{array}$ & $\begin{array}{l}173 \\
144 \\
145 \\
147 \\
184\end{array}$ & $\begin{array}{r}131 \\
114 \\
100 \\
93\end{array}$ & $\begin{array}{l}146 \\
138 \\
132 \\
123 \\
130\end{array}$ & $\begin{array}{l}124 \\
125 \\
117 \\
116 \\
125\end{array}$ & $\begin{array}{l}130 \\
126 \\
132 \\
142 \\
140\end{array}$ & $\begin{array}{l}134 \\
136 \\
128 \\
121 \\
124\end{array}$ & $\overline{---}$ & $\begin{array}{l}79 \\
78 \\
73 \\
80 \\
78\end{array}$ & $\begin{array}{r}114 \\
100 \\
97 \\
104 \\
109\end{array}$ & $\begin{array}{l}132 \\
131 \\
132 \\
133 \\
132\end{array}$ \\
\hline $\begin{array}{l}21 \\
22 \\
23 \\
24 \\
25\end{array}$ & $\begin{array}{l}191 \\
190 \\
177 \\
200 \\
189\end{array}$ & $\begin{array}{l}160 \\
163 \\
167 \\
168 \\
164\end{array}$ & $\begin{array}{l}195 \\
194 \\
196 \\
160 \\
162\end{array}$ & $\begin{array}{r}91 \\
97 \\
112 \\
105 \\
116\end{array}$ & $\begin{array}{l}118 \\
128 \\
132 \\
130 \\
130\end{array}$ & $\begin{array}{l}111 \\
110 \\
109 \\
107 \\
107\end{array}$ & $\begin{array}{l}138 \\
140 \\
140 \\
142 \\
143\end{array}$ & $\begin{array}{l}150 \\
--- \\
--- \\
--- \\
138\end{array}$ & $\begin{array}{l}140 \\
141 \\
167 \\
190 \\
206\end{array}$ & $\begin{array}{l}78 \\
84 \\
90 \\
88 \\
86\end{array}$ & $\begin{array}{l}114 \\
117 \\
120 \\
120 \\
126\end{array}$ & $\begin{array}{l}128 \\
121 \\
122 \\
121 \\
128\end{array}$ \\
\hline $\begin{array}{l}26 \\
27 \\
28 \\
29 \\
30 \\
31\end{array}$ & $\begin{array}{l}210 \\
216 \\
235 \\
-222 \\
214\end{array}$ & $\begin{array}{l}160 \\
153 \\
148 \\
148 \\
146 \\
---\end{array}$ & $\begin{array}{l}148 \\
145 \\
148 \\
149 \\
150 \\
142\end{array}$ & $\begin{array}{l}115 \\
115 \\
116 \\
125 \\
112 \\
116\end{array}$ & $\begin{array}{l}111 \\
122 \\
113 \\
-\square \\
-\cdots\end{array}$ & $\begin{array}{r}109 \\
110 \\
101 \\
98 \\
117\end{array}$ & $\begin{array}{l}144 \\
143 \\
144 \\
143 \\
145 \\
---\end{array}$ & $\begin{array}{l}140 \\
143 \\
142 \\
141 \\
144 \\
143\end{array}$ & $\begin{array}{l}175 \\
145 \\
--- \\
--- \\
---\end{array}$ & $\begin{array}{r}87 \\
87 \\
97 \\
97 \\
96 \\
---\end{array}$ & $\begin{array}{l}125 \\
125 \\
122 \\
121 \\
124 \\
118\end{array}$ & $\begin{array}{l}138 \\
136 \\
130 \\
128 \\
126 \\
--\end{array}$ \\
\hline MEAN & 190 & 170 & 160 & 119 & 129 & 109 & 128 & 139 & --- & 91 & 110 & 128 \\
\hline
\end{tabular}

$\begin{array}{lllllll}\text { WTR YR } 1989 & \text { MEAN } & 136 & \text { MAX } & 235 & \text { MIN } & 73\end{array}$

TEMPERATURE, WATER (DEG. C), WATER YEAR OCTOBER 1988 TO SEPTEMBER 1989

\begin{tabular}{|c|c|c|c|c|c|c|c|c|c|c|c|c|}
\hline DAY & $\alpha C T$ & NOV & DEC & JAN & FEB & MAR & APR & MAY & JUN & JUL & AUG & SEP \\
\hline $\begin{array}{l}1 \\
2 \\
3 \\
4 \\
5\end{array}$ & $\begin{array}{l}28.0 \\
28.0 \\
27.5 \\
25.0 \\
25.0\end{array}$ & $\begin{array}{l}20.0 \\
19.5 \\
19.5 \\
20.5 \\
20.0\end{array}$ & $\begin{array}{l}15.0 \\
14.0 \\
14.0 \\
14.0 \\
14.0\end{array}$ & $\begin{array}{l}13.0 \\
12.5 \\
13.0 \\
12.0 \\
12.0\end{array}$ & $\begin{array}{r}14.5 \\
14.0 \\
13.5 \\
13.5\end{array}$ & $\begin{array}{r}---5 \\
9.5 \\
10.0 \\
12.0 \\
11.5\end{array}$ & $\begin{array}{l}17.5 \\
18.0 \\
18.0 \\
18.5 \\
19.0\end{array}$ & $\begin{array}{l}22.5 \\
23.0 \\
23.0 \\
23.0 \\
23.0\end{array}$ & $\begin{array}{l}27.0 \\
27.0 \\
28.0 \\
28.0 \\
28.0\end{array}$ & $\begin{array}{l}--- \\
--0 \\
26.0 \\
26.0\end{array}$ & $\begin{array}{l}29.0 \\
29.0 \\
30.0 \\
30.0 \\
30.0\end{array}$ & $\begin{array}{l}32.0 \\
32.0 \\
31.5 \\
31.0 \\
32.5\end{array}$ \\
\hline $\begin{array}{r}6 \\
7 \\
8 \\
9 \\
10\end{array}$ & $\begin{array}{l}25.0 \\
24.0 \\
24.0 \\
23.5 \\
23.5\end{array}$ & $\begin{array}{l}20.0 \\
19.5 \\
20.0 \\
19.5 \\
19.0\end{array}$ & $\begin{array}{l}14.0 \\
14.0 \\
14.0 \\
14.0 \\
14.0\end{array}$ & $\begin{array}{r}15.0 \\
12.0 \\
12.0 \\
-.- \\
--\end{array}$ & $\begin{array}{l}13.0 \\
13.0 \\
12.0 \\
12.0 \\
10.0\end{array}$ & $\begin{array}{l}11.0 \\
12.0 \\
11.0 \\
12.0 \\
12.0\end{array}$ & $\begin{array}{l}18.5 \\
18.0 \\
19.0 \\
16.0 \\
15.0\end{array}$ & $\begin{array}{r}23.0 \\
23.0 \\
--0 \\
--0\end{array}$ & $\begin{array}{l}28.0 \\
28.0 \\
27.0 \\
26.5 \\
27.0\end{array}$ & $\begin{array}{l}26.0 \\
26.0 \\
26.0 \\
26.0 \\
26.0\end{array}$ & $\begin{array}{l}30.5 \\
30.0 \\
30.0 \\
30.0 \\
30.0\end{array}$ & $\begin{array}{l}31.0 \\
31.0 \\
31.0 \\
31.5 \\
31.0\end{array}$ \\
\hline $\begin{array}{l}11 \\
12 \\
13 \\
14 \\
15\end{array}$ & $\begin{array}{l}24.0 \\
24.0 \\
22.0 \\
22.0 \\
22.0\end{array}$ & $\begin{array}{l}19.0 \\
19.0 \\
19.0 \\
20.0 \\
20.0\end{array}$ & $\begin{array}{r}13.0 \\
12.0 \\
12.0 \\
12.0 \\
-\end{array}$ & $\begin{array}{r}12.0 \\
12.0 \\
-.- \\
--\end{array}$ & $\begin{array}{l}10.0 \\
10.0 \\
10.0 \\
11.0 \\
12.0\end{array}$ & $\begin{array}{l}13.0 \\
13.0 \\
14.0 \\
15.0 \\
15.0\end{array}$ & $\begin{array}{l}15.0 \\
15.0 \\
15.0 \\
15.0 \\
16.0\end{array}$ & $\begin{array}{l}22.0 \\
22.0 \\
21.5 \\
22.0 \\
22.0\end{array}$ & $\begin{array}{r}27.0 \\
26.5 \\
27.0 \\
26.0\end{array}$ & $\begin{array}{l}27.0 \\
27.5 \\
27.0 \\
27.0 \\
27.5\end{array}$ & $\begin{array}{l}29.0 \\
30.0 \\
29.5 \\
29.0 \\
30.0\end{array}$ & $\begin{array}{l}31.0 \\
31.0 \\
29.5 \\
30.0 \\
29.0\end{array}$ \\
\hline $\begin{array}{l}16 \\
17 \\
18 \\
19 \\
20\end{array}$ & $\begin{array}{l}22.0 \\
22.0 \\
21.5 \\
21.5 \\
21.0\end{array}$ & $\begin{array}{l}20.0 \\
20.0 \\
20.0 \\
20.0 \\
19.0\end{array}$ & $\begin{array}{l}12.0 \\
12.0 \\
12.0 \\
12.0 \\
12.0\end{array}$ & $\begin{array}{r}--- \\
12.0 \\
11.0 \\
12.0\end{array}$ & $\begin{array}{l}12.0 \\
12.0 \\
12.0 \\
12.0 \\
12.5\end{array}$ & $\begin{array}{l}16.0 \\
16.0 \\
16.0 \\
16.0 \\
17.0\end{array}$ & $\begin{array}{l}16.0 \\
17.0 \\
17.0 \\
18.0 \\
18.0\end{array}$ & $\begin{array}{l}21.5 \\
22.0 \\
24.0 \\
23.0 \\
22.0\end{array}$ & $\overline{---}$ & $\begin{array}{l}27.5 \\
28.0 \\
28.0 \\
28.0 \\
28.0\end{array}$ & $\begin{array}{l}30.0 \\
30.0 \\
30.0 \\
30.0 \\
30.0\end{array}$ & $\begin{array}{l}29.0 \\
29.0 \\
29.0 \\
29.0 \\
29.0\end{array}$ \\
\hline $\begin{array}{l}21 \\
22 \\
23 \\
24 \\
25\end{array}$ & $\begin{array}{l}21.0 \\
21.0 \\
21.0 \\
21.0 \\
21.0\end{array}$ & $\begin{array}{l}18.5 \\
18.0 \\
17.5 \\
17.0 \\
17.0\end{array}$ & $\begin{array}{l}12.0 \\
12.0 \\
12.0 \\
12.0 \\
13.0\end{array}$ & $\begin{array}{l}11.0 \\
11.0 \\
11.0 \\
11.0 \\
12.0\end{array}$ & $\begin{array}{l}14.0 \\
13.0 \\
12.0 \\
12.0 \\
11.0\end{array}$ & $\begin{array}{l}17.0 \\
17.0 \\
17.0 \\
16.0 \\
16.0\end{array}$ & $\begin{array}{l}18.0 \\
18.0 \\
19.0 \\
19.0 \\
20.0\end{array}$ & $\begin{array}{r}23.0 \\
--- \\
24.0\end{array}$ & $\begin{array}{l}26.0 \\
26.0 \\
27.0 \\
26.5 \\
27.0\end{array}$ & $\begin{array}{l}28.0 \\
27.5 \\
28.0 \\
28.0 \\
28.0\end{array}$ & $\begin{array}{l}30.0 \\
30.0 \\
31.0 \\
31.0 \\
31.0\end{array}$ & $\begin{array}{l}29.0 \\
29.0 \\
29.0 \\
27.0 \\
26.0\end{array}$ \\
\hline $\begin{array}{l}26 \\
27 \\
28 \\
29 \\
30 \\
31\end{array}$ & $\begin{array}{r}21.0 \\
21.0 \\
21.5 \\
21.0 \\
19.5\end{array}$ & $\begin{array}{l}17.0 \\
17.0 \\
16.0 \\
16.0 \\
16.0 \\
-.-\end{array}$ & $\begin{array}{l}13.0 \\
13.0 \\
13.0 \\
12.0 \\
13.0 \\
13.0\end{array}$ & $\begin{array}{l}12.0 \\
12.0 \\
12.0 \\
11.5 \\
13.0 \\
13.0\end{array}$ & $\begin{array}{r}10.5 \\
11.0 \\
11.0 \\
- \\
-.-\end{array}$ & $\begin{array}{l}16.0 \\
17.0 \\
16.0 \\
-17.0 \\
17.0\end{array}$ & $\begin{array}{r}21.5 \\
22.0 \\
22.0 \\
22.0 \\
22.5 \\
-\end{array}$ & $\begin{array}{l}24.5 \\
25.5 \\
25.5 \\
27.0 \\
26.5 \\
26.0\end{array}$ & $\begin{array}{r}27.0 \\
26.5 \\
-.- \\
--- \\
---\end{array}$ & $\begin{array}{l}28.0 \\
28.0 \\
29.0 \\
29.0 \\
29.0 \\
29.0\end{array}$ & $\begin{array}{l}31.0 \\
31.0 \\
31.0 \\
31.0 \\
31.0 \\
32.0\end{array}$ & $\begin{array}{l}26.0 \\
25.0 \\
25.0 \\
25.0 \\
25.0 \\
\end{array}$ \\
\hline MEAN & 23.0 & 19.0 & 13.0 & -- & 12.0 & 14.5 & 18.0 & 23.5 & -- & 27.5 & 30.0 & 29.0 \\
\hline NTR & & & 20.5 & MAX & 32.5 & & & & & & & \\
\hline
\end{tabular}

